\begin{tabular}{|c|l|}
\hline Title & $\begin{array}{l}\text { A highly stereoselective construction of 1,2-trans } \beta \text {-glycosidic linkages capitalizing on 2-azido-2-deoxy-D-glycosyl } \\
\text { diphenyl phosphates as glycosyl donors }\end{array}$ \\
\hline Author(s) & Tsuda, Toshifumi; Nakamura, Seiichi; Hashimoto, Shunichi \\
\hline Citation & $\begin{array}{l}\text { Tetrahedron, 60(47), 10711-10737 } \\
\text { https:/doi.org/10.1016/.tet.2004.08.076 }\end{array}$ \\
\hline Issue Date & 2004 10-01 \\
\hline Doc URL & http://hdl.handle.net/2115/614 \\
\hline Type & article (author version) \\
\hline File Information & TET13146.pdf \\
\hline
\end{tabular}

Instructions for use 


\section{Graphical Abstract}

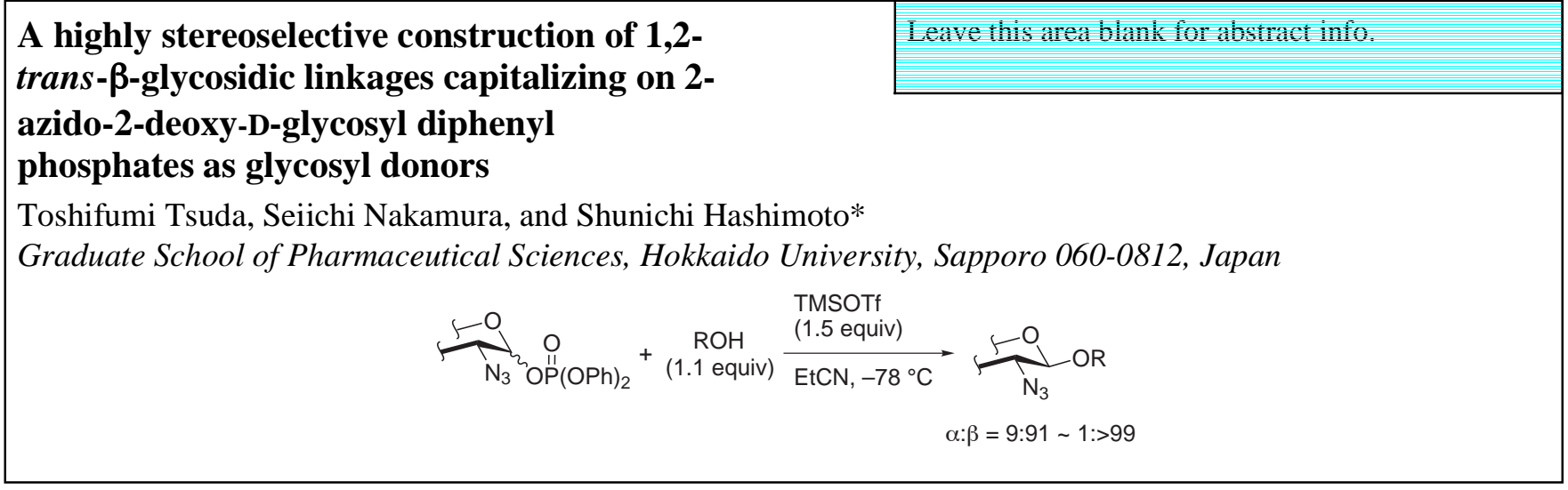




\title{
A highly stereoselective construction of 1,2-trans- $\beta$-glycosidic linkages capitalizing on 2-azido-2-deoxy-D-glycosyl diphenyl phosphates as glycosyl donors
}

\author{
Toshifumi Tsuda, Seiichi Nakamura, and Shunichi Hashimoto* \\ Graduate School of Pharmaceutical Sciences, Hokkaido University, Sapporo 060-0812, Japan
}

\begin{abstract}
The scope of TMSOTf-promoted glycosidation of 2-azido-2-deoxyglycopyranosyl diphenyl phosphates is investigated. The 3,4,6-tri- $O$-benzyl-protected glucosyl and galactosyl donors and the 4,6-O-benzylidene-protected galactosyl donor each react with a range of acceptor alcohols in the presence of a stoichiometric amount of TMSOTf in propionitrile at $-78{ }^{\circ} \mathrm{C}$ to afford 1,2 -trans- $\beta$-linked disaccharides in high yields with $\alpha: \beta$ ratios ranging from 9:91 to 1:>99, regardless of the anomeric composition of the donor used. The use of propionitrile as a solvent at $-78^{\circ} \mathrm{C}$ has proven to be among the best choice for the highest levels of $\beta$-selectivity reported to date for this type of glycosidation. A plausible reaction mechanism, which features a large equilibrium preference for $\alpha$-glycosyl-nitrilium ions over $\beta$ nitrilium ions, is proposed based on byproducts formed through their intermediacy and accounts for the observed excellent $\beta$-selectivities.
\end{abstract}

\section{Introduction}

The rapidly growing significance of glycosides and oligosaccharides as constituents of biologically important compounds such as antitumor antibiotics and glycoconjugates has mandated the rational design and development of stereocontrolled glycosidation reactions. ${ }^{1}$ Since 2acetamido-2-deoxy-D-glycopyranosides, mainly found in $\beta$ glycosidic linkage, are ubiquitous building blocks of glycolipids, glycoproteins, proteoglycans and peptidoglycans, numerous procedures for synthesizing 1,2-trans- $\beta$-linked 2 acetamido-2-deoxy-D-glycosides have been reported. ${ }^{2}$ In terms of efficiency and practicality, direct glycosidation using 2-acetamido-2-deoxyglycosyl donors should constitute an ideal procedure for the stereocontrolled construction of these linkages. In practice, however, the reactions of these donors generally lead to the predominant formation of oxazoline derivatives via a neighboring group participation and subsequent elimination of an amide proton. Although oxazolines can react with acceptor alcohols in the presence of Brønsted or Lewis acids to afford 1,2-trans-glycosides with the natural 2-acetamido group (i.e. oxazoline method), the harsh reaction conditions for this conversion have precluded its wide application for synthesizing complex oligosaccharides. ${ }^{3}$

\footnotetext{
Keywords: 2-azido-2-deoxyglycopyranosyl diphenyl phosphate; $\beta$ selective glycosidation; $\alpha$-nitrilium ion

* Corresponding author. Tel.: +81-11-706-3236; fax: +81-11-706-4981; email: hsmt@pharm.hokudai.ac.jp
}

To overcome this problem, Lemieux and co-workers introduced the use of 2-deoxy-2-phthalimidoglycosyl donors as a reliable method for synthesizing 2-acetamido2 -deoxy- $\beta$-glycosides. ${ }^{4}$ The phthalimido method generally gives high yields and virtually complete $\beta$-selectivity with most glycosyl acceptors as demonstrated with numerous complex oligosaccharide syntheses. However, removing the phthaloyl group requires basic conditions at elevated temperatures, which often cause the product to partially decompose. Therefore, a variety of different 2-amino protecting groups with an anchimeric assistance such as $N$ 2,2,2-trichloroethoxycarbonyl (Troc), ${ }^{5 \mathrm{a}-\mathrm{c}} \mathrm{N}$-allyloxycarbonyl (Alloc), ${ }^{5 \mathrm{c}} N$-benzyloxycarbonyl (Cbz), ${ }^{5 \mathrm{c}} N$-trichloroacetyl (TCA), ${ }^{5 \mathrm{~d}} \mathrm{~N}$-tetrachlorophthaloyl (TCP), ${ }^{5 \mathrm{e}-\mathrm{g}} \mathrm{N}$-dithiasuccinoyl (Dts), ${ }^{5, i} \quad N, N$-diacetyl, ${ }^{5 \mathrm{j}} \mathrm{N}$-4,5-dichlorophthaloyl (DCPhth), ${ }^{5 \mathrm{k}} N$-dimethylmaleoyl (DMM), ${ }^{51} N, N$-dibenzyl, ${ }^{5 \mathrm{~m}}$ and $N$-thiodiglycoloyl (TDG) ${ }^{5 n}$ have been investigated.

An alternative approach to 2 -acetamido-2-deoxy- $\beta$-glycopyranosides involves using 2-azido-2-deoxyglycopyranosyl donors. Although the azido group as a latent amino functionality is incapable of neighboring group participation, modest to high levels of $\beta$-selectivity were observed with 2-azido-2-deoxyglycosyl trichloroacetimidates, ${ }^{6-8} S$ xanthates, ${ }^{9}$ isopropenyl carbonate, ${ }^{10}$ 2-pyridinecarboxylates, ${ }^{11}$ dibutyl phosphates, ${ }^{12}$ and phenylthio glycosides. ${ }^{13}$ Of these, glycosidation of 2-azido-2-deoxyglycosyl trichloroacetimidates in the presence of $\mathrm{BF}_{3} \cdot \mathrm{OEt}_{2}$ in $\mathrm{CH}_{2} \mathrm{Cl}_{2}-$ hexane $^{7}$ or in the presence of TMSOTf in acetonitrile ${ }^{8}$ is the method of choice for a highly stereoselective construction of 2-azido-2-deoxy- $\beta$-glycosides. ${ }^{14}$ 
We recently developed glycosyl donors that incorporate various phosphorus-containing leaving groups. The glycosidations constitute mild and efficient methods for the highly stereocontrolled construction of 1,2-trans- $\beta$ - and 1,2 -cis- $\alpha$-glycosidic linkages with or without a participating group at $\mathrm{C} 2 .{ }^{15}$ The exceptionally high levels of $\beta$ selectivity observed with 2,3,4,6-tetra- $O$-benzyl-protected glycosyl diphenyl phosphates, ${ }^{15 a} N, N, N^{\prime}, N^{\prime}$-tetramethylphosphorodiamidates, ${ }^{15 \mathrm{~d}}$ and diethyl phosphites ${ }^{15 \mathrm{e}}$ suggested that these leaving groups would also be promising candidates for constructing 2-azido-2-deoxy- $\beta$-glycosidic linkages. In this article, the scope, limitations, and mechanism of TMSOTf-promoted glycosidation of 2azido-2-deoxyglycosyl diphenyl phosphates (Eq. (1)) are documented. $^{16}$ In addition, a comparative study with TMSOTf-promoted glycosidation of 2-azido-2-deoxyglycosyl trichloroacetimidates is described.

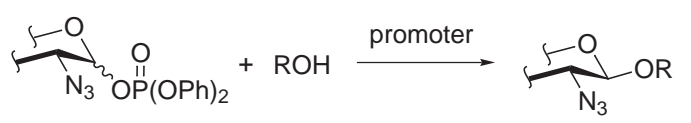

\section{Results and discussion}

\subsection{Preparation of 2-azido-2-deoxy-D-glycosyl donors}

2-Azido-2-deoxy-D-glycosyl donors were prepared according to the standard procedures used for 2,3,4,6-tetra$O$-benzyl-protected glycosyl donors. Application of Sabesan's phosphorylation method ${ }^{17}\left[\mathrm{ClP}(\mathrm{O})(\mathrm{OPh})_{2}\right.$, DMAP, $\left.\mathrm{CH}_{2} \mathrm{Cl}_{2}, \mathrm{O}^{\circ} \mathrm{C}\right]$ to the corresponding glycopyranoses 1a-c and 3a-c afforded 2-azido-2-deoxyglycosyl diphenyl phosphates $\mathbf{2 a}-\mathbf{c}$ and $\mathbf{4 a}-\mathbf{c}$ in good to high yields (Table 1). Diphenyl phosphate 2a with $\alpha: \beta$ ratio of $2: 98$ was obtained by coupling 2-azido-3,4,6-tri- $O$-benzyl-2-deoxy- $\alpha$-Dglucosyl trichloroacetimidate $(5 \alpha)^{7 \mathrm{~b}}$ with diphenyl phosphoric acid in $\mathrm{CH}_{2} \mathrm{Cl}_{2}$ at $0{ }^{\circ} \mathrm{C}$ (Eq. (2)). ${ }^{18}$

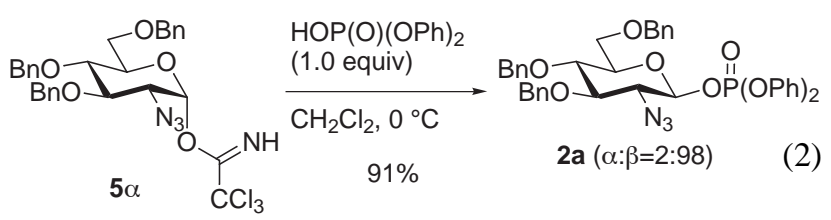

Tetramethylphosphorodiamidate $\mathbf{6}$ was prepared by condensing a lithium alkoxide derived from 1a with bis(dimethylamino)phosphorochloridate in THF-HMPA (Eq. (3)). ${ }^{15 \mathrm{~d}}$ On the other hand, 2-azido-2-deoxyglucosyl diethyl phosphite was inaccessible since it decomposed upon concentration in vacuo, although the reaction of 1a with diethyl chlorophosphite and triethylamine proceeded in $\mathrm{CH}_{2} \mathrm{Cl}_{2}$ at $0{ }^{\circ} \mathrm{C}$. The obtained 2-azido-2-deoxyglycosyl

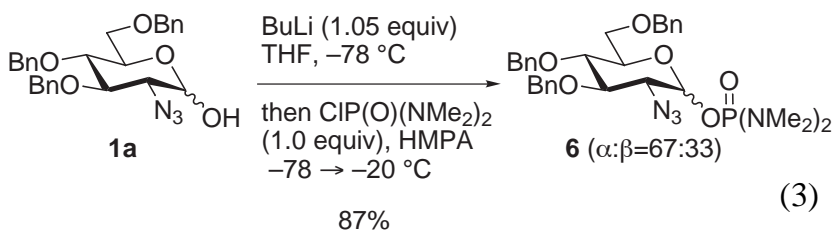

Table 1. Preparation of 2-azido-2-deoxyglycosyl diphenyl phosphates.

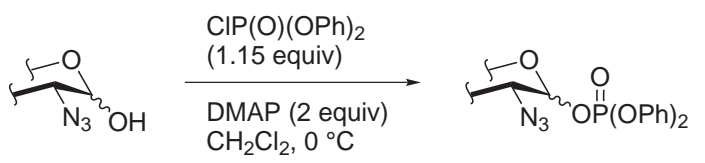

entry

a Determined by $109 \mathrm{MHz}{ }^{31} \mathrm{P}$ NMR using $85 \% \mathrm{H}_{3} \mathrm{PO}_{4}$ as an external standard.

donors were purified by silica gel column chromatography, and stored without decomposition in the freezer (at $-30^{\circ} \mathrm{C}$ ) for several months.

\subsection{Reaction optimization}

At the outset of this study, glycosidations of 2-azido-3,4,6tri- $O$-benzyl-2-deoxy-D-glucosyl diphenyl phosphate $\mathbf{2 a}$ $(\alpha: \beta=72: 28$ or $2: 98)$ and $N, N, N^{\prime}, N^{\prime}$-tetramethylphosphorodiamidate $6(\alpha: \beta=67: 33)$ were explored with $O-6$ - or $O-4$ unprotected glycosides 7 or 8 (1.1 equiv each) as highly reactive and less reactive acceptor alcohols, respectively (Table 2). The addition of a $1.0 \mathrm{M}$ solution of TMSOTf (1.5 equiv) in $\mathrm{CH}_{2} \mathrm{Cl}_{2}$ to a cooled solution $\left(-78{ }^{\circ} \mathrm{C}\right.$ ) of the donor and acceptor in propionitrile afforded a disaccharide and the $\alpha: \beta$ ratio was assayed by HPLC $\left(\right.$ Zorbax $^{\circledR}$ Sil column). As expected from previous work, ${ }^{15 a}$ TMSOTfpromoted glycosidations of the diphenyl phosphate $\mathbf{2 a}$ with 7 or 8 in propionitrile at $-78{ }^{\circ} \mathrm{C}$ proceeded smoothly to give disaccharides 9 and $\mathbf{1 0}$ in high yields with excellent $\beta$ selectivities, regardless of the anomeric composition of the donor (entries 1-4) (Fig. 1). The reactions of phosphorodiamidate 6 under the same conditions exhibited virtually the same $\beta$-selectivities as those found with $\mathbf{2 a}$ (entries 5 and 6), although longer reaction times were required. In 
Table 2. TMSOTf-Promoted glycosidation of 2-azido-2-deoxyglycosyl donors $2 \mathbf{a}^{\mathrm{a}}$ and $\mathbf{6}^{\mathrm{b}}$

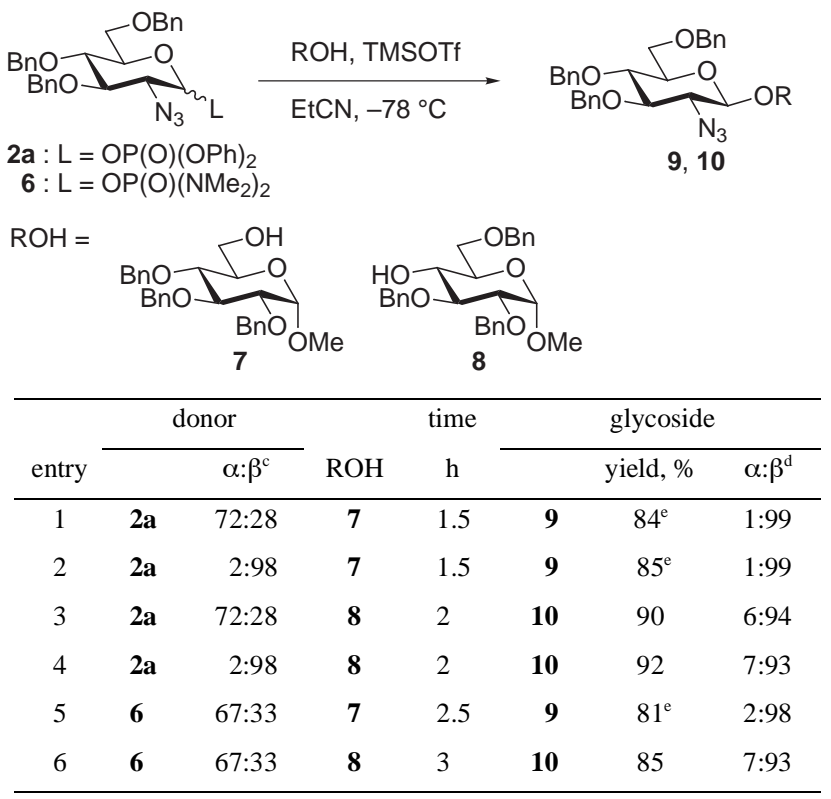

${ }^{a}$ Donor 2a/ROH/TMSOTf molar ratio $=1.0 / 1.1 / 1.5$.

${ }^{\mathrm{b}}$ Donor 6/ROH/TMSOTf molar ratio=1.0/1.1/1.8.

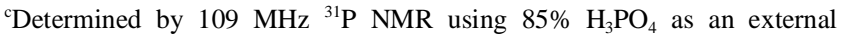
standard.

${ }^{\mathrm{d}}$ The ratio was determined by HPLC (column, Zorbax ${ }^{\circledR}$ Sil, $4.6 \times 250 \mathrm{~mm}$; eluent, $13 \%$ or $17 \% \mathrm{AcOEt}$ in hexane; flow rate $1.0 \mathrm{~mL} / \mathrm{min}$ ).

${ }^{\mathrm{e}} \alpha$-Imidate 11 was obtained in $5-7 \%$ yield.

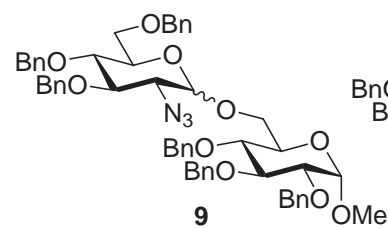

NOE
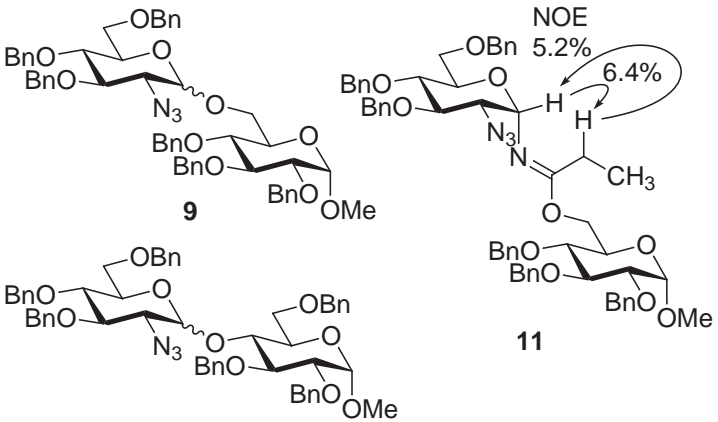

11

10

Figure 1. Products of glycosidation reactions of diphenyl phosphate 2a with 7 and 8 .

either case, the reaction did not go to completion when a substoichiometric amount of TMSOTf was used. Upon further examining these reactions, we were somewhat surprised to find a small amount $(5-7 \%)$ of the hydrolysisprone $\alpha$-imidate 11, which has an $R_{f}$ value comparable to disaccharide 9, was produced as a byproduct when alcohol 7 was used as an acceptor (entries 1,2 and 5). It must be mentioned that imidate byproducts such as $\mathbf{1 1}$ are formed regardless of the nature of 2-azido-2-deoxyglycosyl donors whenever the reactions with highly reactive $O$-6-unprotected glycoside alcohols are conducted in propionitrile (vide infra). Fortunately, their formation did not prevent the isolation of products since the imidates were easily hydro-
Table 3. Effect of solvent in TMSOTf-promoted glycosidation of 2-azido2-deoxyglycosyl diphenyl phosphate $\mathbf{2 a}$.

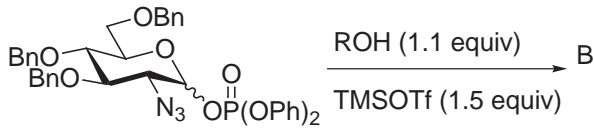

2a $(\alpha: \beta=72: 28)$

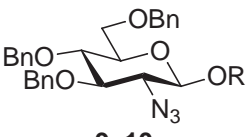

9, 10

\begin{tabular}{cccccccc}
\hline & & & temp. $^{\text {a }}$ & time & \multicolumn{3}{c}{ glycoside } \\
\cline { 6 - 8 } entry & $\mathrm{ROH}$ & solvent & ${ }^{\circ} \mathrm{C}$ & $\mathrm{h}$ & & yield, \% & $\alpha: \beta^{\mathrm{b}}$ \\
\hline 1 & $\mathbf{7}$ & $\mathrm{EtCN}$ & -78 & 1.5 & $\mathbf{9}$ & 84 & $1: 99$ \\
2 & $\mathbf{7}$ & $\mathrm{CH}_{2} \mathrm{Cl}_{2}$ & -78 & 4 & $\mathbf{9}$ & 88 & $1: 99$ \\
3 & $\mathbf{7}$ & toluene & -65 & 2 & $\mathbf{9}$ & 90 & $4: 96$ \\
4 & $\mathbf{7}$ & $\mathrm{EtOAc}$ & -65 & 2 & $\mathbf{9}$ & 89 & $11: 89$ \\
5 & $\mathbf{7}$ & $\mathrm{Et}_{2} \mathrm{O}$ & -30 & 1 & $\mathbf{9}$ & 88 & $38: 62$ \\
6 & $\mathbf{8}$ & $\mathrm{EtCN}$ & -78 & 2 & $\mathbf{1 0}$ & 90 & $6: 94$ \\
7 & $\mathbf{8}$ & $\mathrm{CH}_{2} \mathrm{Cl} l_{2}$ & -78 & 8 & $\mathbf{1 0}$ & 84 & $10: 90$ \\
8 & $\mathbf{8}$ & toluene & -65 & 8 & $\mathbf{1 0}$ & 81 & $24: 76$ \\
\hline
\end{tabular}

a Temperature limit for smooth reaction.

${ }^{\mathrm{b}}$ The ratio was determined by HPLC (column, Zorbax ${ }^{\circledR}$ Sil, $4.6 \times 250 \mathrm{~mm}$; eluent, $13 \%$ or $17 \%$ AcOEt in hexane; flow rate $1.0 \mathrm{~mL} / \mathrm{min}$ ).

lyzed upon an acidic aqueous work-up. The ${ }^{1} \mathrm{H}$ NOE between $\mathrm{H} 1$ ' and $\mathrm{CH}_{2}$ of ethyl group established the anti stereochemistry of 11. In contrast, such a byproduct was not detected when less reactive alcohol $\mathbf{8}$ was used. While the phosphorodiamidate $\mathbf{6}$ has a greater shelf-stability than the diphenyl phosphate 2a, we selected the phosphate method due to the ease in preparing this type of donor.

Examining solvents other than propionitrile for the reaction of diphenyl phosphate $\mathbf{2 a}(\alpha: \beta=72: 28)$ with $O$-6-unprotected glycoside 7 showed that similar high levels of $\beta$ selectivity could be achieved in $\mathrm{CH}_{2} \mathrm{Cl}_{2}$ and toluene (Table 3 , entries 1-5). A further solvent survey with $O$-4-unprotected glycoside $\mathbf{8}$ revealed that propionitrile was optimal for this glycosidation and has a beneficial effect on the

Table 4. Temperature profile of TMSOTf-promoted glycosidation of 2azido-2-deoxyglycosyl diphenyl phosphate $\mathbf{2 a}$.

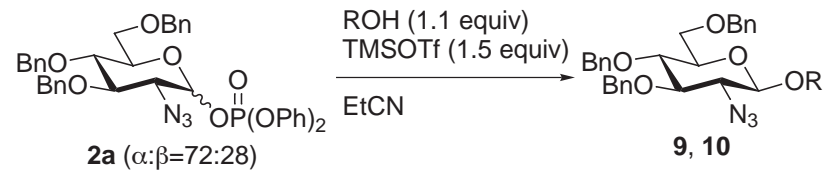

\begin{tabular}{ccccccc}
\hline & & temp. & time & \multicolumn{3}{c}{ glycoside } \\
\cline { 5 - 7 } entry & ROH & ${ }^{\circ} \mathrm{C}$ & $\mathrm{h}$ & & yield, $\%$ & $\alpha: \beta^{\mathrm{a}}$ \\
\hline 1 & $\mathbf{7}$ & -78 & 1.5 & $\mathbf{9}$ & 84 & $1: 99$ \\
2 & $\mathbf{7}$ & -45 & 0.5 & $\mathbf{9}$ & 89 & $3: 97$ \\
3 & $\mathbf{7}$ & -10 & 0.1 & $\mathbf{9}$ & 92 & $8: 92$ \\
4 & $\mathbf{8}$ & -78 & 2 & $\mathbf{1 0}$ & 90 & $6: 94$ \\
5 & $\mathbf{8}$ & -45 & 0.5 & $\mathbf{1 0}$ & 88 & $13: 87$ \\
\hline
\end{tabular}

a The ratio was determined by HPLC (column, Zorbax ${ }^{\circledR} \mathrm{Sil}, 4.6 \times 250 \mathrm{~mm}$; eluent, $13 \%$ or $17 \%$ AcOEt in hexane; flow rate $1.0 \mathrm{~mL} / \mathrm{min}$ ). 
stereoselectivity as well as the reaction rate (entries 6-8). Consistent with the proposal by Schmidt, ${ }^{8}$ an exceptionally high order of $\beta$-selectivity in propionitrile can be explained by the intermediacy of 2-azido-2-deoxy- $\alpha$-D-glucosylnitrilium ion associated with triflate as a counterion (vide infra).

As expected the temperature profile of the glycosidation in propionitrile revealed a descending $\beta$-selectivity with ascending temperature (Table 4). The temperature effect was more pronounced with less reactive alcohol 8 (entries 4 vs 5) than with 7.

\subsection{Glycosidations of 2 -azido-3,4,6-tri- $O$-benzyl-2- deoxyglycosyl diphenyl phosphates $2 \mathrm{a}$ and $4 \mathrm{a}$}

With the optimal reaction conditions determined, glycosidations of 2-azido-3,4,6-tri- $O$-benzyl-2-deoxyglycosyl diphenyl phosphates $\mathbf{2 a}(\alpha: \beta=72: 28)$ and $\mathbf{4 a}(\alpha: \beta=58: 42)$ in the D-gluco and D-galacto series were explored with a range of suitably protected glycoside alcohols (Fig. 2). The results are compiled in Tables 5 and 6 . In all cases, TMSOTf-promoted glycosidations in propionitrile at -78 ${ }^{\circ} \mathrm{C}$ offered a facile and high-yielding entry to 1,2-trans- $\beta$ linked disaccharides, wherein the $\alpha: \beta$ ratios ranged from 9:91 to $1:>99$.

Seeberger and co-workers reported that TMSOTf-promoted coupling of 2-azido-2-deoxyglucosyl dibutyl phosphate with glycoside alcohols $\mathbf{1 2}$ or $\mathbf{1 3}$ in acetonitrile at $-40{ }^{\circ} \mathrm{C}$ produced disaccharides $\mathbf{1 7}$ and $\mathbf{1 8}$ in modest yields with $\alpha: \beta$ ratios of $1: 5$ and $1: 4$, respectively. ${ }^{12}$ Clearly, the present method is superior to the dibutyl phosphate method

Table 5. TMSOTf-Promoted glycosidation of 2-azido-3,4,6-tri- $O$-benzyl2-deoxyglucosyl diphenyl phosphate 2 a with acceptor alcohols. ${ }^{\text {a,b }}$

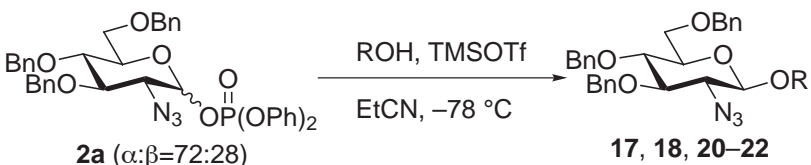

\begin{tabular}{cccccc}
\hline & & time & \multicolumn{3}{c}{ glycoside } \\
\cline { 4 - 6 } entry & ROH & $\mathrm{h}$ & & yield, $\%$ & $\alpha: \beta^{\mathrm{c}}$ \\
\hline 1 & $\mathbf{1 2}$ & 1.5 & $\mathbf{1 7}$ & $79^{\mathrm{d}}$ & $2: 98$ \\
2 & $\mathbf{1 3}$ & 2 & $\mathbf{1 8}$ & 91 & $9: 91$ \\
3 & $\mathbf{1 4}$ & 2 & $\mathbf{2 0}$ & 89 & $1:>99$ \\
4 & $\mathbf{1 5}$ & 2 & $\mathbf{2 1}$ & 90 & $5: 95$ \\
$5^{\text {e }}$ & $\mathbf{1 6}$ & 2 & $\mathbf{2 2}$ & 88 & $7: 93^{\mathrm{f}}$ \\
\hline
\end{tabular}

The reaction was carried out on $0.1 \mathrm{mmol}$ scale.

${ }^{\mathrm{b}}$ Donor 2a/ROH/TMSOTf molar ratio=1.0/1.1/1.5 unless otherwise noted.

${ }^{\mathrm{c}}$ The ratio was determined by HPLC (column, Zorbax ${ }^{\circledR} \mathrm{Sil}, 4.6 \times 250 \mathrm{~mm}$; eluent, 17 20\% AcOEt in hexane or $14 \%$ THF in hexane; flow rate 1.0 $\mathrm{mL} / \mathrm{min}$ ), unless otherwise stated.

${ }^{\mathrm{d}} \alpha$-Imidate 19 was obtained in $9 \%$ yield.

${ }^{\mathrm{e}}$ The reaction was performed with 2.0 equiv of TMSOTf.

${ }^{\mathrm{f}}$ Determined by $500 \mathrm{MHz}{ }^{1} \mathrm{H}$ NMR.
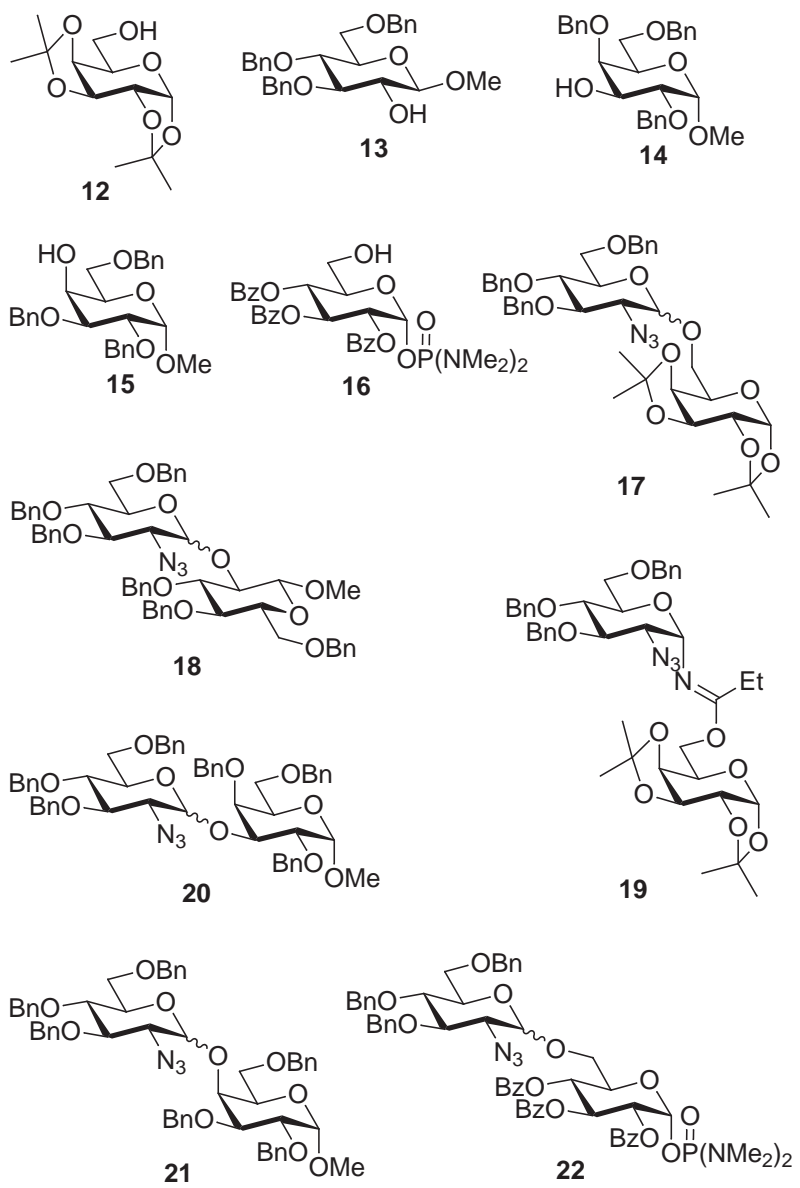

Figure 2. Acceptor alcohols and products in Table 5.

in terms of product yield and stereoselectivity (Table 5, entries 1 and 2). Here again, a small amount (9\%) of $\alpha$ imidate byproduct 19 was detected when $O$-6-unprotected glycoside 12 was used. It is also noteworthy that glycosylation of $O$-3-unprotected galactose derivative 14 exclusively formed disaccharide $\mathbf{2 0} \beta$, which corresponds to GlcNAc $\beta 1 \rightarrow 3 \mathrm{Gal}$, a constituent of biologically important gangliosides such as sialyl Lewis ${ }^{\mathrm{x}}$ (entry 3 ). Since the fully benzoylated glucosyl tetramethylphosphorodiamidate is unaffected at temperatures below $-5{ }^{\circ} \mathrm{C}$ by these reaction conditions, ${ }^{19}$ chemoselective glycosidation was uneventfully realized using $O$-6-unprotected glucosyl phosphorodiamidate $\mathbf{1 6}$ as a disarmed acceptor (entry 5). It is interesting to note that 2-azido-2-deoxygalactosyl diphenyl phosphate $4 \mathbf{a}$ is even more reactive than the corresponding glucosyl donor $\mathbf{2 a}$, as manifested by much shorter reaction times (Table 6). When alcohols 7 and $\mathbf{8}$ were used, donor $\mathbf{4 a}$ displayed somewhat lower and higher $\beta$-selectivities, respectively, than donor $2 \mathrm{a}$. In the former reaction, $5 \%$ of $O$-6-propionyl-protected glycoside 26, due to the hydrolysis of the imidate byproduct (not shown), was obtained. The effectiveness of the present method was also demonstrated by synthesizing LacdiNAc equivalent 29, which was achieved by glycosylation of $O$-4-unprotected glucosamine derivative 24 in $86 \%$ yield with an $\alpha: \beta$ ratio of 8:92 (entry 4) (Fig. 3). 
Table 6. TMSOTf-Promoted glycosidation of 2-azido-3,4,6-tri- $O$-benzyl2-deoxygalactosyl diphenyl phosphate $\mathbf{4 a}$ with acceptor alcohols. ${ }^{a}$
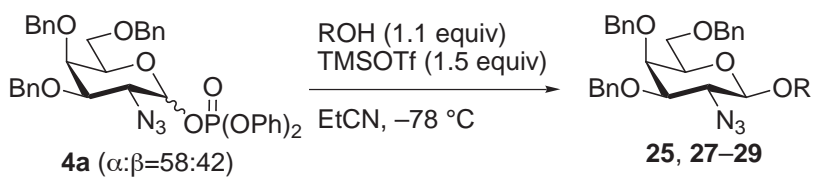

4a $(\alpha: \beta=58: 42)$

25, 27-29

\begin{tabular}{cccccc}
\hline & & time & \multicolumn{3}{c}{ glycoside } \\
\cline { 4 - 6 } entry & ROH & $\mathrm{h}$ & & yield, \% & $\alpha: \beta^{\mathrm{b}}$ \\
\hline 1 & $\mathbf{7}$ & 0.2 & $\mathbf{2 5}$ & $86^{\mathrm{c}}$ & $4: 96$ \\
2 & $\mathbf{8}$ & 0.5 & $\mathbf{2 7}$ & 90 & $4: 96$ \\
3 & $\mathbf{2 3}$ & 0.3 & $\mathbf{2 8}$ & 81 & $6: 94$ \\
4 & $\mathbf{2 4}$ & 0.5 & $\mathbf{2 9}$ & 86 & $8: 92$ \\
\hline
\end{tabular}

The reaction was carried out on $0.1 \mathrm{mmol}$ scale.

${ }^{\mathrm{b}}$ The ratio was determined by HPLC (column, Zorbax ${ }^{\circledR}$ Sil, 4.6×250 mm; eluent, $17 \%$ AcOEt in hexane or $17 \%$ THF in hexane; flow rate 1.0 $\mathrm{mL} / \mathrm{min}$ ).

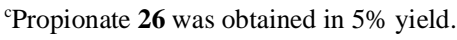<smiles>COC1OC2(C)OC(C)(C)OC1C2O</smiles>

23

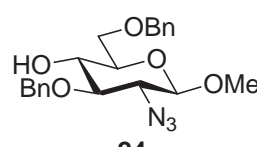

24

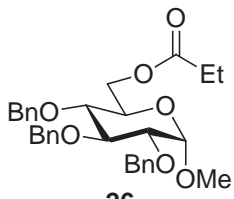

26
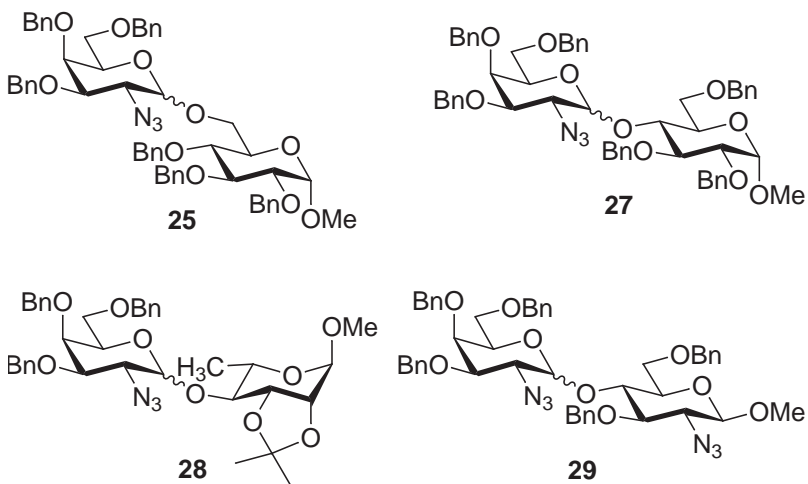

Figure 3. Acceptor alcohols and products in Table 6.

\subsection{Glycosidations of 3,4,6-tri- $O$-acetyl-2-azido-2- deoxyglycosyl diphenyl phosphates $2 \mathrm{~b}$ and $4 \mathrm{~b}$}

While 2-azido-3,4,6-tri- $O$-benzyl-2-deoxyglycosyl donors 2a and 4a performed well, attempts to employ 3,4,6-tri- $O$ acetyl-protected glycosyl donors $\mathbf{2 b}$ and $\mathbf{4 b}$ met with less success. 2-Azido-2-deoxyglucosyl diphenyl phosphate $\mathbf{2 b}$ was activated by TMSOTf at $-65^{\circ} \mathrm{C}$ in propionitrile, but the reaction with alcohol 7 predominantly formed imidates 30 with an $\alpha: \beta$ ratio of $85: 15$ (Table 7, entry 1). Although some of the $\beta$-imidate partially decomposed during column chromatography on silica gel, $\alpha$-imidate $30 \alpha$ was safely isolated in $79 \%$ yield. In this reaction, the corresponding disaccharide 31 with an $\alpha: \beta$ ratio of 14:86 was obtained in only $4 \%$ yield. Likewise, the reaction of 2-azido-2-
Table 7. TMSOTf-Promoted glycosidation of 3,4,6-tri- $O$-acetyl-2-azido2-deoxyglycosyl diphenyl phosphates $\mathbf{2 b}$ and $\mathbf{4 b}$ with alcohol 7.,
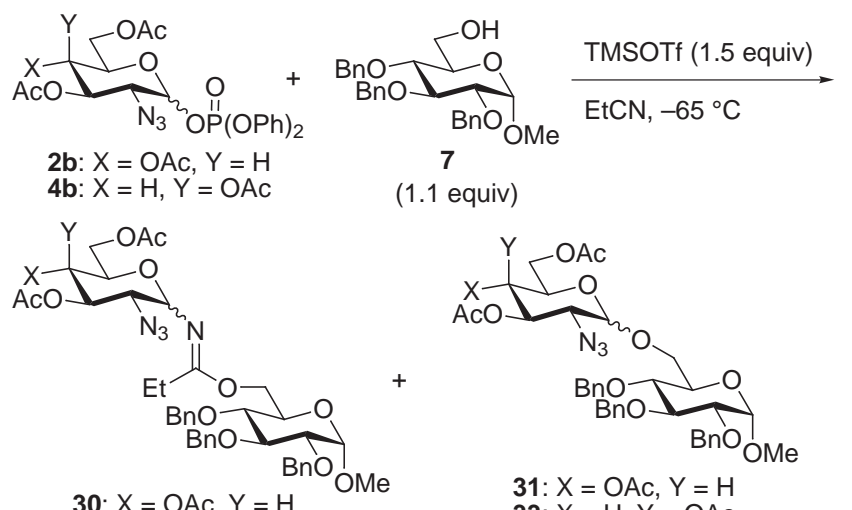

32: $\mathrm{X}=\mathrm{H}, \mathrm{Y}=\mathrm{OAC}$

$X=O A C, Y=H$

33: $\mathrm{X}=\mathrm{H}, \mathrm{Y}=\mathrm{OAC}$

\begin{tabular}{ccccccccc}
\hline & & time & \multicolumn{2}{c}{ imidate } & & \multicolumn{3}{c}{ glycoside } \\
\cline { 8 - 9 } \cline { 6 - 8 } entry & donor & $\mathrm{h}$ & & $\alpha: \beta^{\mathrm{c}}$ & & yield, $\%$ & $\alpha: \beta^{\mathrm{c}}$ \\
\hline 1 & $\mathbf{2 b}$ & 4 & $\mathbf{3 0}^{\mathrm{d}}$ & $85: 15$ & & $\mathbf{3 1}$ & 4 & $14: 86$ \\
2 & $\mathbf{4 b}$ & 3 & $\mathbf{3 2}^{\mathrm{e}}$ & $88: 12$ & & $\mathbf{3 3}$ & 9 & $3: 97$ \\
\hline
\end{tabular}

a The reaction was carried out on $0.1 \mathrm{mmol}$ scale.

${ }^{\mathrm{b}}$ The anomeric $\alpha: \beta$ ratio of the phosphates: $\mathbf{2 b}, 46: 54 ; \mathbf{4 b}, 24: 76$.

${ }^{\mathrm{c}}$ The ratio was determined by HPLC (column, Zorbax ${ }^{\circledR}$ Sil, $4.6 \times 250 \mathrm{~mm}$; eluent, 29\% AcOEt-THF (1:1) in hexane or $20 \%$ THF in hexane; flow rate $1.0 \mathrm{~mL} / \mathrm{min}$ ).

${ }^{\mathrm{d}}$ Only $\alpha$-imidate $\mathbf{3 0} \alpha$ could be isolated in $79 \%$ yield after chromatographic separation.

${ }^{\text {e}}$ Only $\alpha$-imidate $\mathbf{3 2} \alpha$ could be isolated in $68 \%$ yield after chromatographic separation.

deoxygalactosyl diphenyl phosphate $\mathbf{4 b}$ with 7 afforded imidate $32(\alpha: \beta=88: 12)$ as major product, along with $9 \%$ of disaccharide 33 ( $\alpha: \beta=3: 97)$, wherein the $\alpha$-imidate $32 \alpha$ was isolated in $68 \%$ yield (entry 2 ). These disappointing results are attributed to the electron-withdrawing effect of the ester functionality, which deactivates the anomeric reactivity of nitrilium ion intermediates and favors a nucleophilic attack by alcohol $\mathbf{7}$ on a nitrilium carbon leading to imidates $\mathbf{3 0}$ and $\mathbf{3 2}$ (vide infra). Although the fully acyl-protected 2-azido-2-deoxyglycosyl donors are not suitable for the present coupling reaction, other donors with partially acyl protection should not be excluded (vide infra).

\subsection{Glycosidations of 2 -azido-4,6- $O$-benzylidene-2- deoxyglycosyl diphenyl phosphates $2 \mathrm{c}$ and $4 \mathrm{c}$}

It is well documented that 4,6- $O$-benzylidene-protected glycosyl donors exhibit reduced reactivities ${ }^{20}$ and different stereoselectivities ${ }^{21}$ compared to the fully benzylated ones. Therefore, we were driven to investigate glycosidations of 2-azido-4,6-O-benzylidene-2-deoxyglycosyl diphenyl phosphates. 2-Azido-2-deoxyglucosyl donor 2c was activated with TMSOTf at $-45^{\circ} \mathrm{C}$ in propionitrile, but the reaction with 7 gave disaccharide 35 in only $6 \%$ yield with an $\alpha: \beta$ ratio of $8: 92$ and considerable amounts of imidates $36(\alpha: \beta=91: 9)$; the $\alpha$-imidate $\mathbf{3 6} \alpha$ was isolated in $84 \%$ yield (Table 8 , entry 1). In stark contrast, 2-azido-2- 
Table 8. TMSOTf-Promoted glycosidation of 2 -azido-4,6- $O$-benzylidene2-deoxyglycosyl diphenyl phosphates $\mathbf{2 c}$ and $\mathbf{4 c}$ with acceptor alcohols. ${ }^{a}$

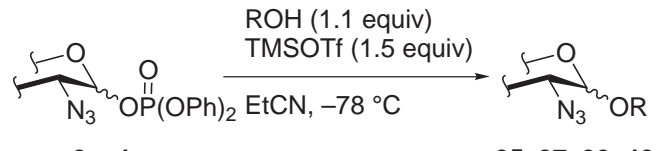

2c, $4 c$

$35,37,39,40$

\begin{tabular}{cccccccc}
\hline & & & time & \multicolumn{3}{c}{ glycoside } \\
\cline { 5 - 7 } entry & donor & ROH & $\mathrm{h}$ & & yield, \% & $\alpha: \beta^{\mathrm{b}}$ \\
\hline $1^{\mathrm{c}}$ & $\mathbf{2}^{\mathrm{d}}$ & $\mathbf{7}$ & 4 & $\mathbf{3 5}$ & $6^{\mathrm{e}}$ & $8: 92$ \\
2 & $\mathbf{4 c}^{\mathrm{f}}$ & $\mathbf{7}$ & 3 & $\mathbf{3 7}$ & $78^{\mathrm{g}}$ & $3: 97$ \\
3 & $\mathbf{4 c}^{\mathrm{f}}$ & $\mathbf{8}$ & 3 & $\mathbf{3 9}$ & 90 & $4: 96$ \\
4 & $\mathbf{4 c}^{\mathrm{f}}$ & $\mathbf{3 4}$ & 2 & $\mathbf{4 0}$ & 80 & $1: 99$ \\
5 & $\mathbf{4 c}^{\mathrm{h}}$ & $\mathbf{3 4}$ & 2 & $\mathbf{4 0}$ & 82 & $2: 98$ \\
\hline
\end{tabular}

a The reaction was carried out on $0.1 \mathrm{mmol}$ scale.

${ }^{\mathrm{b}}$ The ratio was determined by HPLC (column, Zorbax ${ }^{\circledR} \mathrm{Sil}, 4.6 \times 250 \mathrm{~mm}$; eluent, $20 \%$ or $60 \%$ AcOEt in hexane; flow rate $1.0 \mathrm{~mL} / \mathrm{min}$ ).

${ }^{\mathrm{c}}$ The reaction was carried out at $-45^{\circ} \mathrm{C}$.

${ }^{\mathrm{d}} \alpha: \beta=31: 69$.

${ }^{\mathrm{e}} \alpha$-Imidate $36 \alpha$ was obtained in $84 \%$ yield.

${ }^{\mathrm{f}} \alpha: \beta=95: 5$.

${ }^{\mathrm{g}} \alpha$-Imidate 38 was obtained in $10 \%$ yield.

${ }^{\mathrm{h}} \alpha: \beta=0: 100$.
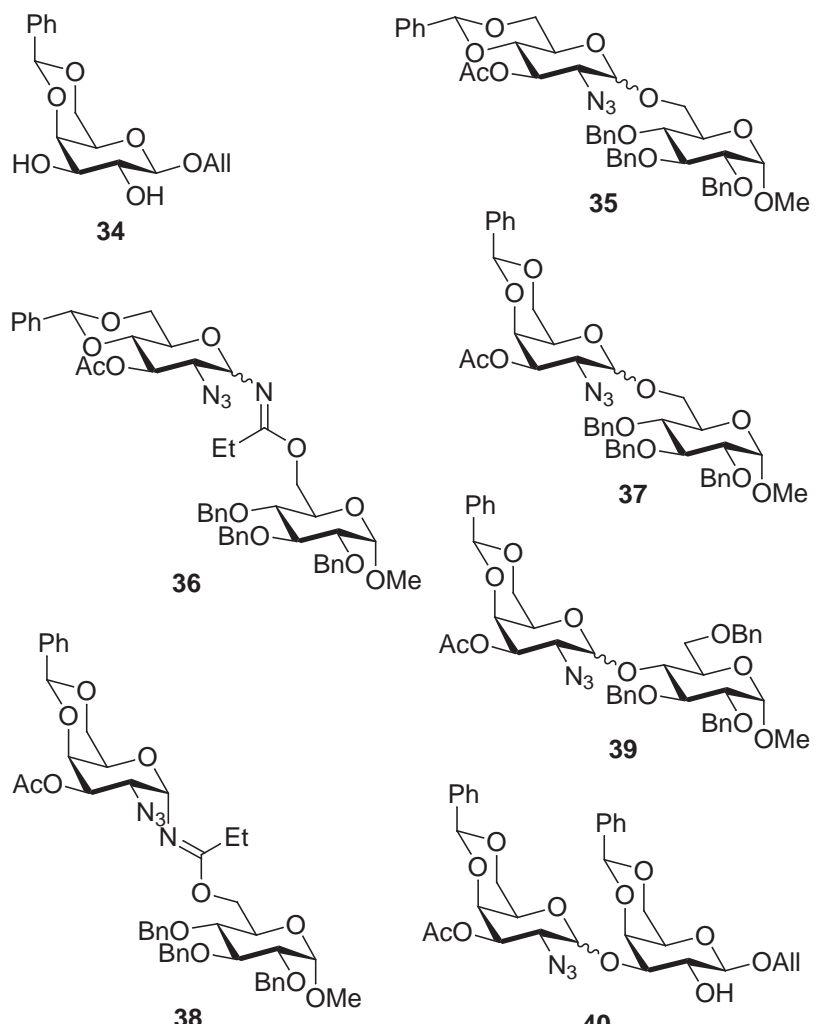

40

Figure 4. Acceptor alcohols and products in Table 8. deoxygalactosyl diphenyl phosphate 4c underwent a smooth coupling with a range of alcohols even at $-78{ }^{\circ} \mathrm{C}$ to provide disaccharides 37, 39, 40 in good yields with excellent $\beta$-selectivities (entries 2-4), although a small amount $(10 \%)$ of $\alpha$-imidate $\mathbf{3 8}$ was produced as a byproduct of the reaction with 7 . It is noteworthy that glycosylation of diol 34 produced 1,2-trans- $\beta$-linked disaccharide 40 with essentially perfect regioselectivity and excellent stereoselectivity $(\alpha: \beta=1: 99$ and $2: 98$ ) (entries 4 and 5). ${ }^{22}$ The difference in reaction mode between these donors may be explained by considering that 2-azido-2deoxyglucosyl donor $\mathbf{2 c}$ is a trans-fused bicyclic compound whereas 2-azido-2-deoxygalactosyl donor $\mathbf{4 c}$ has a relatively flexible, cis-decaline-like architecture (Fig. 4). The greater conformational rigidity of $\mathbf{2 c}$ relative to $\mathbf{4 c}$ would serve to torsionally disarm the nitrilium ion intermediate with respect to formation of the $O$-glycosidic linkage..$^{20,23}$

\subsection{Comparative study}

While high yields and excellent $\beta$-selectivities were achieved in the reactions of glycosyl diphenyl phosphates $\mathbf{2 a}, \mathbf{4 a}$, and $\mathbf{4 c}$ with a range of acceptor alcohols, limitations of the phosphate method were recognized with donors $\mathbf{2 b}$, $\mathbf{2 c}$, and $\mathbf{4 b}$. To verify the effectiveness of the phosphate method, the scope of TMSOTf-promoted glycosidations of the corresponding trichloroacetimidates was examined. Although the exceptional power of the trichloroacetimidate method developed by Schmidt has been well demonstrated in numerous aminosugar-containing oligosaccharide syntheses, ${ }^{24}$ a systematic investigation has yet to be described. The glycosidations were performed under frequently used conditions [cat. TMSOTf, acetonitrile, $-40{ }^{\circ} \mathrm{C}$ ] ${ }^{8,22,24}$ Table 9 summarizes the results. TMSOTf ( 0.1 equiv)-catalyzed glycosidations of trichloroacetimidates $5 \alpha^{7 \mathrm{~b}}$ or $\mathbf{5} \beta^{25}$ with alcohols $\mathbf{7}$ and $\mathbf{8}$ proceeded to completion within $20 \mathrm{~min}$, yielding high levels of $\beta$-selectivity similar to those of $\mathbf{2 a}$ in propionitrile at $-45^{\circ} \mathrm{C}$ (entries 1 and 2 vs entry 2 in Table 4, and entries 3 and 4 vs entry 5 in Table 4). The stereochemical outcome observed was independent of the anomeric configuration of the donor similar to the phosphates. Somewhat surprisingly, evidence of the formation of imidate byproduct such as $\mathbf{1 1}$ could not be detected when alcohol 7 was used. Instead, a small amount (4\%) of $\beta$-trichloroacetamide $\mathbf{4 1}$ was obtained as a byproduct of the reaction of $\mathbf{5} \alpha$ with 7 (entry 1), whereas 41 was not formed from $5 \beta$ (entry 2 ). ${ }^{26}$ A moderate product yield in the reaction of $\mathbf{5} \alpha$ with less reactive alcohol $\mathbf{8}$ was due to the formation of $\beta$-trichloroacetamide 41 (28\%) and lactol 1a (4\%) (entry 3). The yield of byproduct 41 decreased to $10 \%$ with $\mathbf{5} \beta$, thereby allowing a higher product yield (entry 4). A significant improvement in product yield $(68 \% \rightarrow 84 \%)$ was achieved when the reaction of $\mathbf{5} \beta$ with $\mathbf{8}$ was carried out in the presence of MS4A, whereas the beneficial effect was not observed with $5 \alpha$ (entries 5 and 6). Although discrepancies between the behavior of $\alpha$ - and $\beta$-glycosyl trichloroacetimidates were observed in some cases, ${ }^{27}$ the reason is currently unclear.

Next, glycosidation of trichloroacetimidates $5 \alpha$ or $\mathbf{5} \beta$ with alcohol $\mathbf{8}$ in propionitrile at $-78{ }^{\circ} \mathrm{C}$ were explored in order 
Table 9. TMSOTf-Catalyzed glycosidation of 2-azido-2-deoxyglucosyl trichloroacetimidates $\mathbf{5} \alpha$ and $\mathbf{5} \beta$ with alcohols $\mathbf{7}$ and $\mathbf{8}$ in acetonitrile. ${ }^{a}$

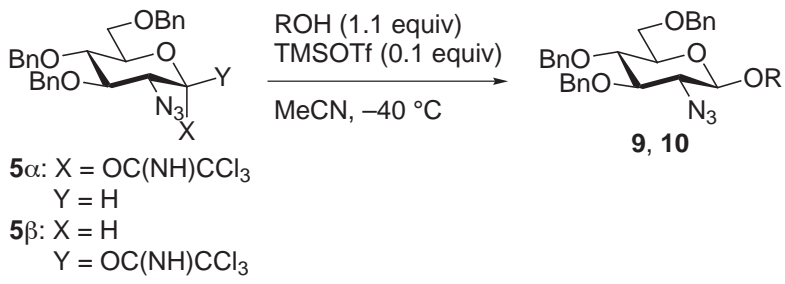

\begin{tabular}{ccccccc}
\hline & & & time & \multicolumn{3}{c}{ glycoside } \\
\cline { 6 - 7 } entry & donor & ROH & h & & yield, \% & $\alpha: \beta^{\mathrm{b}}$ \\
\hline 1 & $\mathbf{5} \alpha$ & $\mathbf{7}$ & 0.1 & $\mathbf{9}$ & $82^{\mathrm{c}}$ & $3: 97$ \\
2 & $\mathbf{5} \beta$ & $\mathbf{7}$ & 0.1 & $\mathbf{9}$ & 85 & $3: 97$ \\
3 & $\mathbf{5} \alpha$ & $\mathbf{8}$ & 0.3 & $\mathbf{1 0}$ & $50^{\mathrm{d}}$ & $10: 90$ \\
4 & $\mathbf{5} \beta$ & $\mathbf{8}$ & 0.3 & $\mathbf{1 0}$ & $68^{\mathrm{e}}$ & $11: 89$ \\
$5^{\mathrm{f}}$ & $\mathbf{5} \alpha$ & $\mathbf{8}$ & 0.3 & $\mathbf{1 0}$ & $51^{\mathrm{g}}$ & $12: 88$ \\
$6^{\mathrm{f}}$ & $\mathbf{5} \beta$ & $\mathbf{8}$ & 0.3 & $\mathbf{1 0}$ & 84 & $12: 88$
\end{tabular}

a The reaction was carried out on $0.1 \mathrm{mmol}$ scale.

${ }^{\text {b}}$ The ratio was determined by HPLC (column, Zorbax ${ }^{\circledR}$ Sil, $4.6 \times 250 \mathrm{~mm}$; eluent, $17 \%$ AcOEt in hexane; flow rate $1.0 \mathrm{~mL} / \mathrm{min}$ ).

${ }^{\mathrm{c}} \beta$-Trichloroacetamide $\mathbf{4 1}$ was obtained in $4 \%$ yield.

${ }^{\mathrm{d}}$ Amide 41 and lactol 1a were obtained in $28 \%$ and $4 \%$ yields, respectively. ${ }^{\mathrm{e}}$ Amide 41 and lactol 1a were obtained in $10 \%$ and 5\% yields, respectively. ${ }_{\mathrm{f}}^{\mathrm{f}}$ In the presence of MS4A.

${ }^{\mathrm{g}}$ Amide 41 was obtained in $35 \%$ yield.<smiles>O=C(NC1C(O)C(OBr)C(OCc2ccccc2)C(OCc2ccccc2)C1[Mg])C(Cl)(Cl)Cl</smiles>

to determine whether the $\beta$-selectivity $(\alpha: \beta=12: 88 \sim 10: 90)$ observed in acetonitrile at $-40{ }^{\circ} \mathrm{C}$ could be enhanced to the ratio $(\alpha: \beta=6: 94)$ achieved with the phosphate method. Although the goal in terms of stereoselectivity could be virtually achieved using 0.2 equiv of TMSOTf, product yields were not preparatively useful (Table 10, entries 1 and 2). When using 1.5 equiv of TMSOTf, product yields from $\mathbf{5} \alpha$ and $\mathbf{5} \beta$ were improved to $54 \%$ and $85 \%$, respectively, without affecting the stereoselectivity (entries 3 and 4). $\alpha$-Amidine byproduct $\mathbf{4 2}$ was obtained in $20 \%$ yield when $\mathbf{5} \alpha$ was used, but $\beta$-trichloroacetamide $\mathbf{4 1}$ was not formed from either $\mathbf{5} \alpha$ or $\mathbf{5} \beta$. It is noteworthy that the formation of an amidine byproduct has not been reported in glycosidation reactions using trichloroacetimidates as glycosyl donors. It is also interesting that the reaction of $\mathbf{5} \alpha$ with 8 in acetonitrile at $-40{ }^{\circ} \mathrm{C}$ gave $\beta$-trichloroacetamide 41 as a major byproduct, whereas the same reaction in propionitrile at $-78{ }^{\circ} \mathrm{C}$ afforded $\alpha$-amidine 42 as a major one, but the reason is unclear. These results again demonstrated the superiority of donor $\mathbf{5} \beta$ over $\mathbf{5} \alpha$. From the results with 4a, 2-azido-2-deoxygalactosyl trichloroacetimidates $43 \alpha^{7 a}$ and $43 \beta^{28}$ are anticipated to have greater reactivities than the corresponding glucosyl donors $\mathbf{5} \alpha$ and
Table 10. TMSOTf-Promoted glycosidation of 2-azido-2-deoxyglucosyl trichloroacetimidates $\mathbf{5} \alpha$ and $\mathbf{5} \beta$ with alcohol $\mathbf{8}$ in propionitrile. ${ }^{\mathrm{a}}$

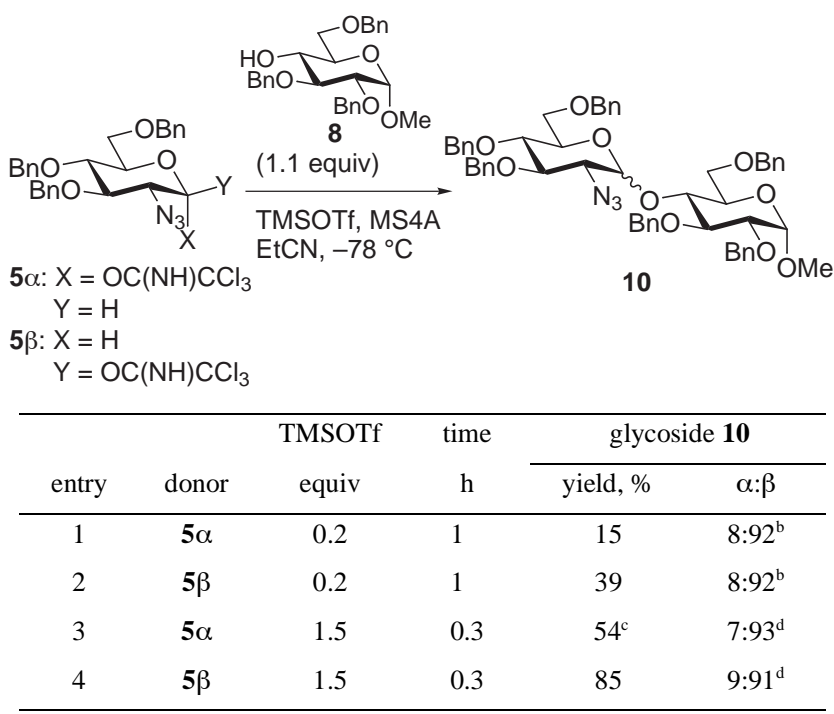

${ }^{a}$ The reaction was carried out on $0.1 \mathrm{mmol}$ scale.

${ }^{\mathrm{b}}$ Determined by $500 \mathrm{MHz}{ }^{1} \mathrm{H}$ NMR.

${ }^{\mathrm{C}}$ Amidine $\mathbf{4 2}$ was obtained in $20 \%$ yield.

${ }^{\mathrm{d}}$ Determined by HPLC (column, Zorbax ${ }^{\circledR}$ Sil, $4.6 \times 250 \mathrm{~mm}$; eluent, $17 \%$ AcOEt in hexane; flow rate $1.0 \mathrm{~mL} / \mathrm{min}$ ).<smiles>CCC(=O)N(C(=N)N(C(=O)C(Cl)(Cl)Cl)C(O)COCc1ccccc1)C(O)COc1ccccc1</smiles>

$5 \beta$. Indeed, the reactions with alcohol $\mathbf{8}$ in propionitrile at $-78{ }^{\circ} \mathrm{C}$ in the presence of 1.5 equiv of TMSOTf proceeded to completion within $5 \mathrm{~min}$ (Table 11). Although virtually the same $\beta$-selectivities as those observed with phosphate 4a were achieved, the product yields (48\% from $43 \alpha$ and $66 \%$ from $43 \beta$ ) were unsatisfactory (Tables 11 vs entry 2 in Table 6), due to the inevitable formation of $\beta$-trichloroacetamide 44 (37\% from $43 \alpha$ and $7 \%$ from $43 \beta$ ) and $\alpha$ amidine 45 (7\% from $43 \alpha$ and $7 \%$ from $43 \beta$ ).

Two key findings emerged from this comparative study. (1) 2-Azido-2-deoxyglycosyl trichloroacetimidates generally exhibit higher reactivities than the corresponding diphenyl phosphates. (2) Only using $\beta$-imidates gives coupling products in good to high yields and with exceptionally high levels of $\beta$-selectivity comparable to those found with an anomeric mixture of diphenyl phosphates, when the reactions are conducted in the presence of 1.5 equiv of TMSOTf in propionitrile at $-78^{\circ} \mathrm{C} .^{29}$

\subsection{Mechanistic considerations}

The beneficial effect of nitrile as a solvent on 1,2-trans- $\beta$ glycosidations without neighboring participation observed by Noyori and co-workers in $1984^{30}$ is now a wellappreciated phenomenon in carbohydrate chemistry. In 
Table 11. TMSOTf-Promoted glycosidation of 2-azido-2-deoxygalactosyl trichloroacetimidates $43 \alpha$ and $43 \beta$ with alcohol 8 in propionitrile. ${ }^{a}$

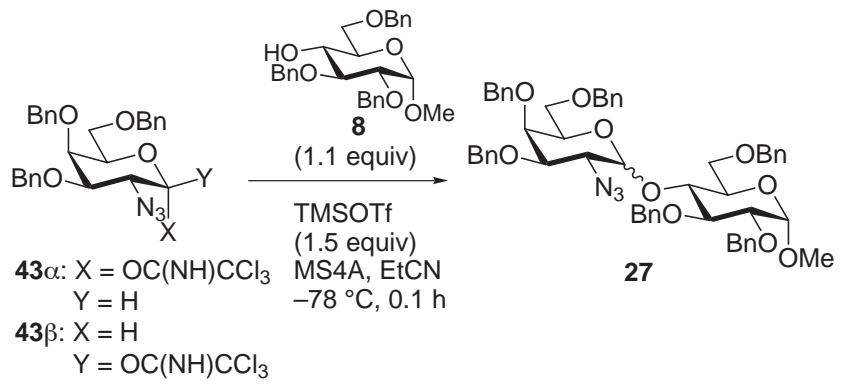

\begin{tabular}{cccccc}
\hline \multirow{2}{*}{ entry } & \multirow{2}{*}{ donor } & \multicolumn{2}{c}{ glycoside $\mathbf{2 7}$} & $\mathbf{4 4}$ & $\mathbf{4 5}$ \\
\cline { 3 - 4 } & yield, \% & $\alpha: \beta^{\mathrm{b}}$ & yield, \% & yield, \% \\
\hline 1 & $\mathbf{4 3} \alpha$ & 48 & $4: 96$ & 37 & 7 \\
2 & $\mathbf{4 3} \beta$ & 66 & $4: 96$ & 7 & 7 \\
\hline
\end{tabular}

${ }^{\mathrm{a}}$ The reaction was carried out on $0.1 \mathrm{mmol}$ scale.

${ }^{\mathrm{b}}$ The ratio was determined by HPLC (column, Zorbax ${ }^{\circledR} \mathrm{Sil}, 4.6 \times 250 \mathrm{~mm}$; eluent, $17 \%$ AcOEt in hexane; flow rate $1.0 \mathrm{~mL} / \mathrm{min}$ ).
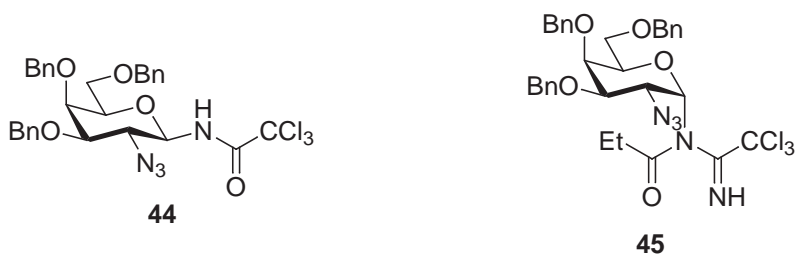

1990 Fraser-Reid ${ }^{31}$ and $\mathrm{Schmidt}^{8}$ separately proposed that the $\beta$-selectivity could be given by an $\mathrm{S}_{\mathrm{N}} 2$-like displacement at the anomeric carbon of kinetically formed $\alpha$-nitrilium ion, which has been widely accepted. ${ }^{32}$ It is evident that the so-called "nitrile effect" plays a pivotal role in the TMSOTf-promoted glycosidations with 2-azido-2deoxyglycosyl diphenyl phosphates since propionitrile at $-78{ }^{\circ} \mathrm{C}$ is an excellent solvent for high levels of $\beta$ selectivity. ${ }^{33}$ Scheme 1 outlines the possible reaction pathways. Diphenyl phosphate $\mathbf{4 6}$ is activated by silylation on the phosphoryl oxygen atom to cleave off the phosphate group, producing oxocarbenium ion $\mathbf{4 8}$ as a common intermediate. Intermediate $\mathbf{4 8}$ is rapidly trapped by propionitrile to form an anomeric mixture of nitrilium ions $49 \alpha$ and $49 \beta$ associated with triflate as a counterion. In this step, the $\alpha$-nitrilium ion $49 \alpha$ preferentially forms over $49 \beta$ because of the stereoelectronically favored axial attack of propionitrile from the $\alpha$-face. ${ }^{34}$ In addition, $49 \alpha$ benefits from anomeric stabilization. ${ }^{35}$ On the kinetic and thermodynamic grounds, the equilibrium between these nitrilium ions would heavily lie to $49 \alpha$. The $S_{\mathrm{N}} 2$-like displacement by acceptor alcohols at the anomeric carbon of $49 \alpha$ and $49 \beta$ affords glycosides $50 \beta$ and $50 \alpha$, respectively, whereas capture of $49 \alpha$ and $49 \beta$ by alcohols at the nitrilium carbon leads to the formation of imidate byproducts $51 \alpha$ and $51 \beta$, respectively. The chemoselectivity depends on the anomeric reactivity of glycosylnitrilium ions 49 influenced by the choice of protecting groups on 2-azido-2-deoxy-sugar components as well as the reactivity of acceptor alcohols, as is demonstrated by

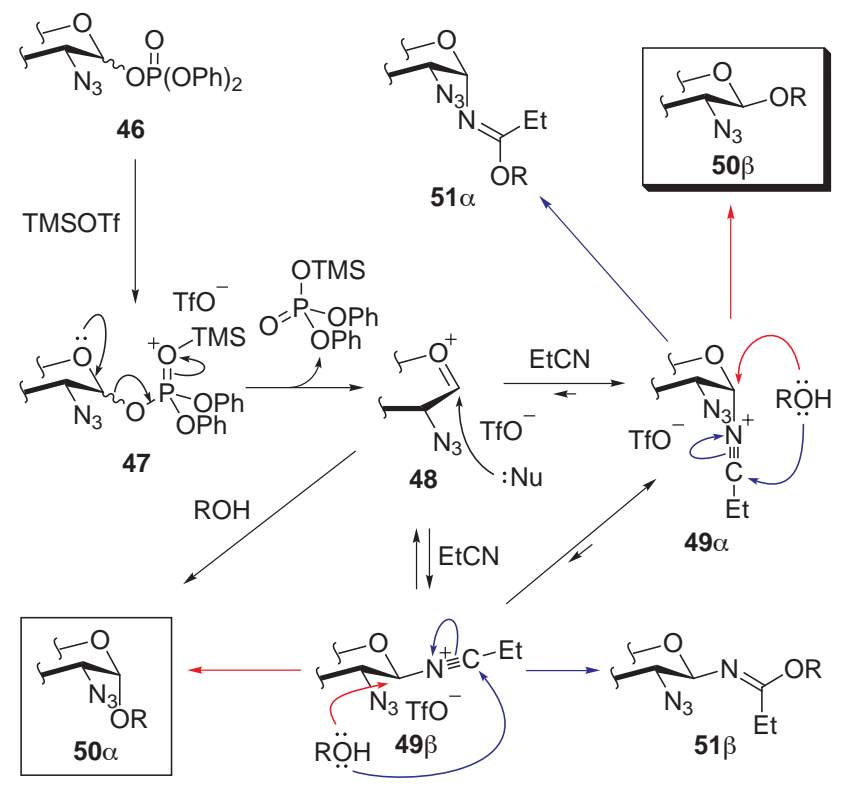

Scheme 1. A mechanistic rationale for TMSOTf-promoted glycosidation of 2-azido-2-deoxyglycosyl diphenyl phosphates.

the foregoing experimental results. The exclusive formation of disaccharides was realized when the 3,4,6-tri- $O$-benzylprotected glucosyl and galactosyl donors $\mathbf{2 a}$ and $\mathbf{4 a}$, and the 4,6- $O$-benzylidene-protected galactosyl donor $\mathbf{4} \mathbf{c}$ were used, although a small amount of imidates was produced as byproducts in the reaction with highly reactive $O-6$ unprotected glycoside alcohols. Hence, the high levels of $\beta$ selectivity observed here are attributed to a large equilibrium preference for $49 \alpha$ as well as a high propensity of $49 \alpha$ for an $S_{\mathrm{N}} 2$-like displacement. ${ }^{6 c}$ The stereochemical reaction course via a common oxocarbenium ion 48 is consistent with the fact that the stereoselectivities are irrespective of the anomeric configuration of the diphenyl phosphates used. Actually, it was found that glycosidation of 2-azido-3,4,6-tri- $O$-benzyl-2-deoxy-D-glucosyl diphenyl phosphate 2a $(\alpha: \beta=2: 98)$ competes with the anomerization to $\alpha$-phosphate via an internal return of the departing diphenyl phosphate group under the present reaction conditions. Of prime importance in terms of a mechanism is that only $\alpha$-imidates $\mathbf{1 1}, \mathbf{1 9}$, and $\mathbf{3 8}$ were obtained as byproducts $(5-10 \%)$ in the reaction of $\mathbf{2 a}$ or $\mathbf{4 c}$ with $O-6-$ unprotected alcohols. Assuming that the much less stable $\beta$-nitrilium ion $49 \beta$ would have a reactivity comparable to $49 \alpha$ toward the imidate formation, these results, along with the ${ }^{1} \mathrm{H}$ NMR analysis of the crude reaction mixture, which did not detect traces of $\beta$-imidates or their hydrolysates, provide evidence that $\alpha$-nitrilium ion $49 \alpha$ exclusively forms at least in these reactions. Along with the finding that the proportion of 1,2-cis- $\alpha$-linked disaccharides slightly increased with less reactive alcohols compared to highly reactive ones, it seems likely that their formation would arise from the kinetically favored $\alpha$-axial attack of alcohols on the transient, solvent separated oxocarbenium ion $\mathbf{4 8}$ rather than the $\mathrm{S}_{\mathrm{N}} 2$-like displacement of any $49 \beta$. While the corresponding imidate byproduct could not be detected due to its increased hydrolytic lability for 3,4,6-tri- $O$-benzylprotected galactosyl donor 4a, highly efficient glycosi- 
dations of $\mathbf{4 a}$ are also assumed to proceed in a similar manner as those of $\mathbf{2 a}$ and $\mathbf{4 c}$. On the other hand, the behaviors of 3,4,6-tri- $O$-acetyl-protected glycosyl donors $\mathbf{2 b}$ and $\mathbf{4 b}$, and 4,6- $O$-benzylidene-protected glucosyl donor $\mathbf{2 c}$ are quite different from those of glycosyl donors $\mathbf{2 a}, \mathbf{4 a}$ and $\mathbf{4 c}$ mentioned above. Those reactions with alcohol $\mathbf{7}$ produced an anomeric mixture of imidates $30(\alpha: \beta=85: 15)$, $32(\alpha: \beta=88: 12)$, and $36(\alpha: \beta=91: 9)$ as main products, along with small amounts of disaccharides $\mathbf{3 1}, \mathbf{3 3}$, and $\mathbf{3 5}$ with $\alpha: \beta$ ratios of $14: 86,3: 97$, and $8: 92$, respectively. It is interesting to note that the $\alpha: \beta$ ratios of imidates $\mathbf{3 0}$ and $\mathbf{3 6}$ in the D-gluco series are opposite to those of the corresponding disaccharides $\mathbf{3 1}$ and 35, respectively. These relationships strongly suggest that glycosidations with electronically or torsionally disarmed 2-azido-2-deoxyglucosyl diphenyl phosphates proceed via an $\mathrm{S}_{\mathrm{N}} 2$-like displacement, where the glucosyl-nitrilium ions $\mathbf{4 9}$ would be too stable to generate the solvent separated oxocarbenium ion $\mathbf{4 8}$. However, this is not the case with 3,4,6-tri- $O$-acetylprotected galactosyl diphenyl phosphate $\mathbf{4 b}$ probably because the galactosyl nitrilium ions $\mathbf{4 9}$ may exhibit greater anomeric reactivities to allow for a dynamic equilibrium than the glucosyl counterparts. ${ }^{20 c, d}$

Since TMSOTf-promoted glycosidations of 2-azido-2deoxyglycosyl trichloroacetimidates exhibit essentially the same high $\beta$-selectivities as those found with diphenyl phosphates under identical conditions, the stereochemical reaction course seems to be analogous to that proposed with the phosphate method in Scheme 1. However, the product yields in the trichloroacetimidate method highly depends on the anomeric configuration of the starting donor and the reactivity of acceptor alcohols. Substantial amounts of $\beta$-trichloroacetamides and $\alpha$-amidines were frequently obtained as byproducts when $\alpha$-trichloroacetimidates were

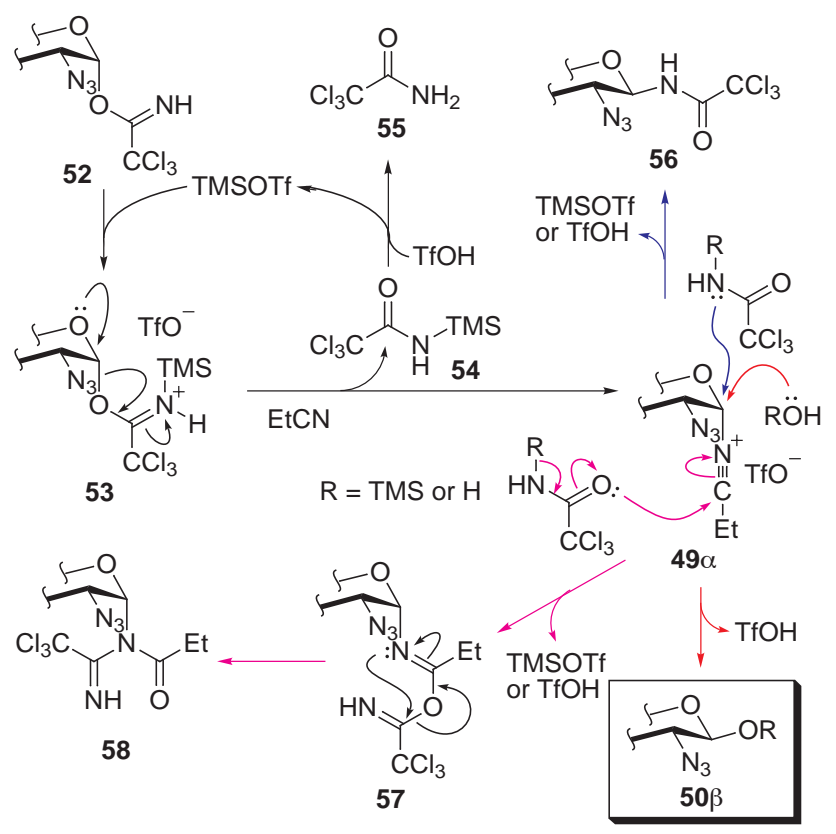

Scheme 2. Potential pathways in the TMSOTf-promoted glycosidation of 2-azido-2-deoxyglycosyl trichloroacetimidates. used as glycosyl donors. Although the striking difference between the behavior of $\alpha$ - and $\beta$-trichloroacetimidates currently cannot be explained, the formation of $\beta$-trichloroacetamides 56 and $\alpha$-amidines 58 can be rationalized by the mechanism shown in Scheme 2. In glycosidations with trichloroacetimidates, the departing trichloroacetamide (55) and/or its TMS derivative $\mathbf{5 4}$ competes as a nucleophile with acceptor alcohols. No such reactions were observed in the phosphate method due to the low nucleophilicity of the diphenyl phosphate. The $\mathrm{S}_{\mathrm{N}} 2$-like displacement by the amide nitrogen atom of $\mathbf{5 4}$ or $\mathbf{5 5}$ at the anomeric carbon of $\alpha$-nitrilium ion $49 \alpha$ leads to $\beta$-amides 56 with inversion of configuration, whereas the capture of $49 \alpha$ by the amide oxygen atom of $\mathbf{5 4}$ or $\mathbf{5 5}$ at the nitrilium carbon followed by rearrangement produces $\alpha$-amidines $\mathbf{5 8}$. The stereocontrolled formation of $\beta$-amides 56 and $\alpha$-amidines 58 again demonstrates the virtually exclusive intermediacy of $\alpha$-nitrilium ion $49 \alpha$ in glycosidations of the 3,4,6-tri- $O$ benzyl-protected glucosyl and galactosyl donors.

\section{Conclusion}

The effectiveness of the diphenyl phosphate group as a leaving group of 2-azido-2-deoxyglycosyl donors has been demonstrated. We found that coupling of the 3,4,6-tri- $O$ benzyl-protected glucosyl and galactosyl donors and the 4,6- $O$-benzylidene-protected galactosyl donor with a range of glycoside alcohols in the presence of 1.5 equiv of TMSOTf in propionitrile at $-78{ }^{\circ} \mathrm{C}$ proceeds smoothly to give 1,2-trans- $\beta$-linked disaccharides in high yields with $\alpha: \beta$ ratios ranging from $9: 91$ to $1:>99$, regardless of the anomeric composition of the starting donor. The use of propionitrile as a solvent at $-78{ }^{\circ} \mathrm{C}$ proved to be the best choice for the highest levels of $\beta$-selectivity reported to date for this type of glycosidation. However, limitations of the phosphate method were recognized for 3,4,6-tri- $O$ acetyl-protected glucosyl and galactosyl donors and 4,6-Obenzylidene-protected glucosyl donor. These results indicate that the properly choosing of protecting groups on 2-azido-2-deoxy-sugar components is crucial for the success in the present method. It has also been experimentally demonstrated that highly efficient and $\beta$ selective glycosidations proceed through intermediate $\alpha$ glycosyl-nitrilium ions followed by an $S_{N} 2$-like displacement, which is based on the finding that only $\alpha$-imidates formed through their intermediate were small amounts of byproducts when highly reactive $O$-6-unprotected glycoside alcohols were used as a glycosyl acceptor. A comparative study with the corresponding trichloroacetimidates under the present reaction conditions demonstrated that similar high levels of $\beta$-selectivity are observed, but the phosphate method generally gives higher product yields than the trichloroacetimidate method. The latter method is frequently accompanied by side-products that originate from the departing trichloroacetamide, particularly when $\alpha$-imidates are used. While the discrepancy in reaction mode between $\alpha$ - and $\beta$-trichloroacetimidates remains to be elucidated, only using $\beta$-trichloroacetimidates ensures a successful result. Thus, the present 
method would be a potent alternative to Schmidt's trichloroacetimidate procedure.

\section{Experimental}

General. Melting points were determined on a Büchi 535 digital melting point apparatus and were uncorrected. Optical rotations were recorded on a JASCO P-1030 digital polarimeter. Infrared (IR) spectra were recorded on a JASCO FT/IR-5300 spectrophotometer and absorbance bands are reported in wavenumber $\left(\mathrm{cm}^{-1}\right)$. Proton nuclear magnetic resonance $\left({ }^{1} \mathrm{H}\right.$ NMR) spectra were recorded on a Bruker ARX500 (500 MHz) spectrometer with tetramethylsilane $\left(\delta_{\mathrm{H}} \quad 0.00\right)$ as an internal standard. Coupling constants $(J)$ are reported in hertz $(\mathrm{Hz})$. Abbreviations of multiplicity are as follows: s, singlet; $d$, doublet; t, triplet; q, quartet; m, multiplet; br, broad. Data are presented as follows: chemical shift, multiplicity, coupling constants, integration and assignment. Carbon nuclear magnetic resonance $\left({ }^{13} \mathrm{C}\right.$ NMR) spectra were recorded on JEOL AL400 (100 MHz) or Bruker ARX500 $(126 \mathrm{MHz})$ spectrometers with $\mathrm{CDCl}_{3}\left(\delta_{\mathrm{C}} 77.0\right)$ as an internal standard. Phosphorus nuclear magnetic resonance $\left({ }^{31} \mathrm{P}\right.$ NMR) spectra were recorded on JEOL EX270 (109 $\mathrm{MHz})$ or Bruker ARX500 (202 MHz) spectrometers with $\mathrm{H}_{3} \mathrm{PO}_{4} \quad\left(\delta_{\mathrm{P}} 0.00\right)$ as an external standard. Fast atom bombardment (FAB) mass spectra were obtained on a JEOL JMS HX110 spectrometer in the Center for Instrumental Analysis, Hokkaido University.

Column chromatography was carried out on Kanto silica gel $60 \mathrm{~N}(40-50 \mu \mathrm{m}$ or $63-210 \mu \mathrm{m})$ or Wakogel C-200 $(75-150 \mu \mathrm{m})$. Analytical thin layer chromatography (TLC) was carried out on Merck Kieselgel $60 \mathrm{~F}_{254}$ plates. Visualization was accomplished with ultraviolet light and anisaldehyde or phosphomolybdic acid stain, followed by heating. HPLC analyses were performed on a JASCO PU980 and UV-970 (detector, $\lambda=254 \mathrm{~nm}$ ). Retention times $\left(t_{\mathrm{R}}\right)$ and peak ratios were determined with a Shimadzu Chromatopac C-R6A. Hexane was HPLC grade, and filtered and degassed prior to use.

Reagents and solvents were purified by standard means or used as received unless otherwise noted. Dehydrated stabilizer free THF was purchased from Kanto Chemical Co., Inc. Dichloromethane and propionitrile were distilled from $\mathrm{P}_{2} \mathrm{O}_{5}$, and redistilled from calcium hydride prior to use. Molecular sieves 4A was finely ground in mortar and heated in vacuo at $220^{\circ} \mathrm{C}$ for $12 \mathrm{~h}$.

All reactions were conducted under an argon atmosphere. Lactols $\mathbf{1 a},{ }^{7 \mathrm{~b}} \mathbf{1 b},,^{7 \mathrm{a}} \mathbf{3 a},,^{7 \mathrm{a}} \mathbf{3} \mathbf{b}^{36}$ and $\mathbf{3} \mathbf{c}^{37}$ were prepared according to literature procedures. For full characterization, most of authentic $\alpha$-glycosides were prepared by glycosidations of diphenyl phosphates with acceptor alcohols in $\mathrm{Et}_{2} \mathrm{O}$ at $0{ }^{\circ} \mathrm{C}$, followed by chromatographic separation from the $\beta$-glycosides. Glycosides $\mathbf{3 1}, \mathbf{3 3}$ and $\mathbf{3 5}$ were prepared by reactions of diphenyl phosphates with alcohol 7 in $\mathrm{CH}_{2} \mathrm{Cl}_{2}$ at $-30{ }^{\circ} \mathrm{C}$, followed by column chromatography.

\subsection{Preparation of 2-azido-2-deoxy-D-glycosyl donors}

4.1.1. Typical procedure for preparation of 2-azido-2deoxyglycopyranosyl diphenyl phosphate: 2-azido-3,4,6tri- $O$-benzyl-2-deoxy-D-glucopyranosyl diphenyl phosphate (2a).

Diphenylphosphoryl chloride $(0.55 \mathrm{~mL}, 2.66 \mathrm{mmol})$ was added to a stirred solution of $1 \mathbf{a}^{7 \mathrm{~b}}(1.10 \mathrm{~g}, 2.31 \mathrm{mmol})$ and DMAP (564 mg, $4.62 \mathrm{mmol})$ in $\mathrm{CH}_{2} \mathrm{Cl}_{2}(10 \mathrm{~mL})$ at $0{ }^{\circ} \mathrm{C}$. After $0.5 \mathrm{~h}$, the reaction was quenched with crushed ice, followed by stirring at room temperature for $15 \mathrm{~min}$. The mixture was poured into a two-layer mixture of $\mathrm{Et}_{2} \mathrm{O}(20$ $\mathrm{mL})$ and saturated aqueous $\mathrm{NaHCO}_{3}(20 \mathrm{~mL})$, and the whole was extracted with AcOEt $(40 \mathrm{~mL})$. The organic layer was washed with brine $(2 \times 20 \mathrm{~mL})$, and dried over anhydrous $\mathrm{Na}_{2} \mathrm{SO}_{4}$. Filtration and evaporation in vacuo furnished the pale yellow oil $(1.70 \mathrm{~g})$, which was purified by column chromatography (silica gel $30 \mathrm{~g}, 2: 1$ hexane/ AcOEt with $\left.2 \% \mathrm{Et}_{3} \mathrm{~N}\right)$ to give diphenyl phosphate $\mathbf{2 a}(1.59$ $\mathrm{g}, 97 \%, \alpha: \beta=72: 28)$ as a colorless oil. The anomeric $\alpha: \beta$ ratio of the diphenyl phosphate was determined by ${ }^{31} \mathrm{P}$ NMR.

Data for $\alpha$-anomer (2a $\alpha)$ : TLC $R_{f}=0.42(2: 1$ hexane/ $\mathrm{AcOEt}) ;[\alpha]_{\mathrm{D}}{ }^{14}+38.1^{\circ}\left(c\right.$ 1.14, $\left.\mathrm{CHCl}_{3}\right)(\alpha: \beta=85: 15)$; IR (film) 3022, 2872, 2870, 2116, 1591, 1491, 1288, 1059, 1188, $966 \mathrm{~cm}^{-1}$; ${ }^{1} \mathrm{H}$ NMR (500 MHz, $\mathrm{CDCl}_{3}$ ) $\delta 3.35$ (brd, $J$ $=10.9 \mathrm{~Hz}, 1 \mathrm{H}, \mathrm{H}-6 \mathrm{a}), 3.61\left(\mathrm{ddd}, J=3.4,9.8,3.3\left(J_{\mathrm{H}-\mathrm{P}}\right) \mathrm{Hz}\right.$, $1 \mathrm{H}, \mathrm{H}-2), 3.64$ (dd, $J=1.0,10.9 \mathrm{~Hz}, 1 \mathrm{H}, \mathrm{H}-6 \mathrm{~b}), 3.79-3.85$ (m, 2H, H-4, H-5), 3.88 (m, 1H, H-3), 4.43 (d, J=11.1 Hz, $1 \mathrm{H}, \mathrm{OCHPh}), 4.536$ (d, $J=11.1 \mathrm{~Hz}, 1 \mathrm{H}, \mathrm{OCHPh}), 4.537$ (d, $J=10.9 \mathrm{~Hz}, 1 \mathrm{H}, \mathrm{OCHPh}), 4.78(\mathrm{~d}, J=10.9 \mathrm{~Hz}, 1 \mathrm{H}$, OCHPh), $4.82(\mathrm{~d}, J=10.7 \mathrm{~Hz}, 1 \mathrm{H}, \mathrm{OCHPh}), 4.86(\mathrm{~d}, J=$ $10.7 \mathrm{~Hz}, 1 \mathrm{H}, \mathrm{OCHPh}), 5.98\left(\mathrm{dd}, J=3.4,6.1\left(J_{\mathrm{H}-\mathrm{P}}\right) \mathrm{Hz}, 1 \mathrm{H}\right.$, $\mathrm{H}-1)$, 7.15-7.35 (m, 25H, Ar-H); ${ }^{13} \mathrm{C}$ NMR (126 MHz, $\left.\mathrm{CDCl}_{3}\right) \delta 63.5\left(\mathrm{~d}, J_{\mathrm{C}-\mathrm{P}}=8.6 \mathrm{~Hz}\right), 67.4,73.2,73.5,75.1$, $75.6,80.0,97.3\left(\mathrm{~d}, J_{\mathrm{C}-\mathrm{P}}=6.3 \mathrm{~Hz}, \mathrm{C}-1\right), 120.1\left(\mathrm{~d}, J_{\mathrm{C}-\mathrm{P}}=5.0\right.$ $\mathrm{Hz}), 120.3\left(\mathrm{~d}, J_{\mathrm{C}-\mathrm{P}}=5.0 \mathrm{~Hz}\right), 125.4,125.5,127.7,127.77$, $127.83,127.9,128.0,128.1,128.4,128.45,128.48,129.7$, $129.8,137.6,137.66,137.70,150.38\left(\mathrm{~d}, J_{\mathrm{C}-\mathrm{P}}=7.5 \mathrm{~Hz}\right)$, $150.44\left(\mathrm{~d}, J_{\mathrm{C}-\mathrm{P}}=7.5 \mathrm{~Hz}\right) ;{ }^{31} \mathrm{P} \mathrm{NMR}\left(109 \mathrm{MHz}, \mathrm{CDCl}_{3}\right) \delta$ -13.3; FAB-HRMS $\mathrm{m} / \mathrm{z}$ calcd for $\mathrm{C}_{39} \mathrm{H}_{39} \mathrm{~N}_{3} \mathrm{O}_{8} \mathrm{P}(\mathrm{M}+\mathrm{H})^{+}$ 708.2474, found 708.2476; Anal. calcd for: $\mathrm{C}_{39} \mathrm{H}_{38} \mathrm{~N}_{3} \mathrm{O}_{8} \mathrm{P}: \mathrm{C}$, $66.19 ; \mathrm{H}, 5.41 ; \mathrm{N}, 5.94$, found $\mathrm{C}, 66.06 ; \mathrm{H}, 5.54 ; \mathrm{N}, 5.82$. Data for $\beta$-anomer $(\mathbf{2 a} \beta)$ : TLC $R_{f}=0.38$ (2:1 hexane/ $\mathrm{AcOEt}) ;[\alpha]_{\mathrm{D}}^{22}+2.69^{\circ}\left(c 1.33, \mathrm{CHCl}_{3}\right)(\alpha: \beta=5: 95)$; IR (film) 3022, 2872, 2870, 2116, 1591, 1491, 1288, 1059, 1188, $966 \mathrm{~cm}^{-1}$; ${ }^{1} \mathrm{H}$ NMR (500 MHz, $\mathrm{CDCl}_{3}$ ) $\delta$ 3.47-3.54 $(\mathrm{m}, 3 \mathrm{H}, \mathrm{H}-2, \mathrm{H}-4, \mathrm{H}-5), 3.63$ (dd, $J=1.7,11.1 \mathrm{~Hz}, 1 \mathrm{H}, \mathrm{H}-$ 6a), 3.73 (dd, $J=3.6,11.1 \mathrm{~Hz}, 1 \mathrm{H}, \mathrm{H}-6 \mathrm{~b}), 3.76$ (t, $J=9.1$ $\mathrm{Hz}, 1 \mathrm{H}, \mathrm{H}-3), 4.45$ (d, $J=12.0 \mathrm{~Hz}, 1 \mathrm{H}, \mathrm{OCHPh}), 4.55$ (d, $J$ $=12.0 \mathrm{~Hz}, 1 \mathrm{H}, \mathrm{OCHPh}), 4.58(\mathrm{~d}, J=10.9 \mathrm{~Hz}, 1 \mathrm{H}, \mathrm{OCHPh})$, $4.78(\mathrm{~d}, J=10.9 \mathrm{~Hz}, 1 \mathrm{H}, \mathrm{OCHPh}), 4.82(\mathrm{~d}, J=11.0 \mathrm{~Hz}, 1 \mathrm{H}$, OCHPh), $4.86(\mathrm{~d}, J=11.0 \mathrm{~Hz}, 1 \mathrm{H}, \mathrm{OCHPh}), 5.15(\mathrm{dd}, J=$ 7.3, $\left.7.3\left(J_{\mathrm{H}-\mathrm{P}}\right) \mathrm{Hz}, 1 \mathrm{H}, \mathrm{H}-1\right), 7.16-7.34(\mathrm{~m}, 25 \mathrm{H}, \mathrm{Ar}-\mathrm{H}) ;{ }^{13} \mathrm{C}$ NMR $\left(126 \mathrm{MHz}, \mathrm{CDCl}_{3}\right) \delta 66.5\left(\mathrm{~d}, J_{\mathrm{C}-\mathrm{P}}=9.2 \mathrm{~Hz}\right), 67.9$, 73.6, 75.0, 75.7, 75.9, 82.9, $98.2\left(\mathrm{~d}, J_{\mathrm{C}-\mathrm{P}}=5.5 \mathrm{~Hz}, \mathrm{C}-1\right)$, $120.1\left(\mathrm{~d}, J_{\mathrm{C}-\mathrm{P}}=5.0 \mathrm{~Hz}\right), 120.5\left(\mathrm{~d}, J_{\mathrm{C}-\mathrm{P}}=5.0 \mathrm{~Hz}\right), 125.5$, 125.6 , 127.68, 127.71, 127.8, 127.9, 128.0, 128.1, 128.4, 
$128.46,128.48,129.6,129.8,137.6,137.7,137.9,150.3$, (d, $\left.J_{\mathrm{C}-\mathrm{P}}=7.5 \mathrm{~Hz}\right), 150.5\left(\mathrm{~d}, J_{\mathrm{C}-\mathrm{P}}=7.5 \mathrm{~Hz}\right) ;{ }^{31} \mathrm{P} \mathrm{NMR}(109$ $\left.\mathrm{MHz}, \quad \mathrm{CDCl}_{3}\right) \quad \delta-13.5$; FAB-HRMS $\mathrm{m} / \mathrm{z}$ calcd for $\mathrm{C}_{39} \mathrm{H}_{39} \mathrm{~N}_{3} \mathrm{O}_{8} \mathrm{P}(\mathrm{M}+\mathrm{H})^{+}$708.2474, found 708.2490.

\subsubsection{3,4,6-Tri- $O$-acetyl-2-azido-2-deoxy-D-glucopyran-} osyl diphenyl phosphate (2b). The reaction was performed according to the typical procedure $\left(10 \mathrm{~mL} \mathrm{CH}_{2} \mathrm{Cl}_{2}, 0{ }^{\circ} \mathrm{C}\right.$, $0.5 \mathrm{~h})$ employing lactol $\mathbf{1 b}^{7 \mathrm{a}}(754 \mathrm{mg}, 2.28 \mathrm{mmol})$, diphenylphosphoryl chloride (0.66 $\mathrm{mL}, 3.19 \mathrm{mmol})$, and DMAP (557 mg, $4.56 \mathrm{mmol})$. The crude product $(1.53 \mathrm{~g}$ ) was purified by column chromatography (silica gel $40 \mathrm{~g}$, 1.5:1 hexane/AcOEt with $1 \% \quad \mathrm{Et}_{3} \mathrm{~N}$ ) to give diphenyl phosphate $2 \mathbf{b}(1.28 \mathrm{~g}, 99 \%, \alpha: \beta=46: 54)$ as a pale yellow syrup. TLC $R_{f}=0.50(1: 1$ hexane/AcOEt $) ;[\alpha]_{\mathrm{D}}{ }^{18}+48.0^{\circ}(c$ $\left.1.35, \mathrm{CHCl}_{3}\right)(\alpha: \beta=46: 54)$; IR (film) 2116, 1753, 1591, 1489, 1188, $970 \mathrm{~cm}^{-1}$; ${ }^{1} \mathrm{H}$ NMR $\left(500 \mathrm{MHz}, \mathrm{CDCl}_{3}\right) \delta 1.99$ (s, $\left.1.5 \mathrm{H}, \mathrm{CH}_{3} \mathrm{CO}\right), 2.02\left(\mathrm{~s}, 1.5 \mathrm{H}, \mathrm{CH}_{3} \mathrm{CO}\right), 2.03(\mathrm{~s}, 1.5 \mathrm{H}$, $\left.\mathrm{CH}_{3} \mathrm{CO}\right), 2.04\left(\mathrm{~s}, 1.5 \mathrm{H}, \mathrm{CH}_{3} \mathrm{CO}\right), 2.08\left(\mathrm{~s}, 1.5 \mathrm{H}, \mathrm{CH}_{3} \mathrm{CO}\right)$, $2.10\left(\mathrm{~s}, 1.5 \mathrm{H}, \mathrm{CH}_{3} \mathrm{CO}\right), 3.64(\mathrm{~m}, 0.5 \mathrm{H}, \mathrm{H}-2 \beta), 3.72$ (ddd, $J$ $\left.=3.4,10.4,3.3\left(J_{\mathrm{H}-\mathrm{P}}\right) \mathrm{Hz}, 0.5 \mathrm{H}, \mathrm{H}-2 \alpha\right), 3.76(\mathrm{~m}, 0.5 \mathrm{H}, \mathrm{H}-$ $5 \beta), 3.80(\mathrm{dd}, J=2.1,12.6 \mathrm{~Hz}, 0.5 \mathrm{H}, \mathrm{H}-6 \mathrm{a \alpha}), 4.00$ (dd, $J=$ $2.3,12.5 \mathrm{~Hz}, 0.5 \mathrm{H}, \mathrm{H}-6 \mathrm{a} \beta$ ), 4.03 (ddd, $J=2.1,3.9,10.4 \mathrm{~Hz}$, $0.5 \mathrm{H}, \mathrm{H}-5 \alpha$ ), 4.17 (dd, $J=3.9,12.6 \mathrm{~Hz}, 0.5 \mathrm{H}, \mathrm{H}-6 \mathrm{~b} \alpha), 4.22$ $(\mathrm{dd}, J=4.8,12.5 \mathrm{~Hz}, 0.5 \mathrm{H}, \mathrm{H}-6 \mathrm{~b} \beta), 5.02-5.11(\mathrm{~m}, 1.5 \mathrm{H}$, $\mathrm{H}-4 \alpha, \mathrm{H}-3 \beta, \mathrm{H}-4 \beta), 5.24$ (dd, $J=7.8,7.8\left(J_{\mathrm{H}-\mathrm{P}}\right) \mathrm{Hz}, 0.5 \mathrm{H}$, $\mathrm{H}-1 \beta), 5.45$ (dd, $J=9.9,10.4 \mathrm{~Hz}, 0.5 \mathrm{H}, \mathrm{H}-3 \alpha), 6.01$ (dd, $J$ $\left.=3.4,6.4\left(J_{\mathrm{H}-\mathrm{P}}\right) \mathrm{Hz}, 0.5 \mathrm{H}, \mathrm{H}-1 \alpha\right), 7.20-7.38(\mathrm{~m}, 10 \mathrm{H}, \mathrm{Ar}-$ $\mathrm{H}) ;{ }^{13} \mathrm{C}$ NMR $\left(126 \mathrm{MHz}, \mathrm{CDCl}_{3}\right) \delta 20.36,20.38,20.41$, $20.5,60.8,60.9\left(\mathrm{~d}, J_{\mathrm{C}-\mathrm{P}}=8.9 \mathrm{~Hz}\right), 61.2,63.9\left(\mathrm{~d}, J_{\mathrm{C}-\mathrm{P}}=9.8\right.$ $\mathrm{Hz}), 67.4,67.6,69.7,70.3,72.4,72.5,96.1\left(\mathrm{~d}, J_{\mathrm{C}-\mathrm{P}}=5.5\right.$ $\mathrm{Hz}, \mathrm{C}-1 \alpha), 97.6\left(\mathrm{~d}, J_{\mathrm{C}-\mathrm{P}}=5.0 \mathrm{~Hz}, \mathrm{C}-1 \beta\right), 119.88,119.92$, $120.0,120.15,120.19,120.23,120.3,125.56,125.62,129.6$, $129.7,129.8,150.0,150.06,150.10,150.12,150.15,150.16$, 169.36, 169.39, 169.5, 169.7, 170.2; ${ }^{31} \mathrm{P}$ NMR (109 MHz, $\left.\mathrm{CDCl}_{3}\right) \delta-13.6(\beta),-13.2(\alpha)$; FAB-HRMS $m / z$ calcd for $\mathrm{C}_{24} \mathrm{H}_{27} \mathrm{~N}_{3} \mathrm{O}_{11} \mathrm{P}(\mathrm{M}+\mathrm{H})^{+}$564.1383, found 564.1379; Anal. calcd for: $\mathrm{C}_{24} \mathrm{H}_{26} \mathrm{~N}_{3} \mathrm{O}_{11} \mathrm{P}: \mathrm{C}, 51.16 ; \mathrm{H}, 4.65 ; \mathrm{N}, 7.46$, found C, 51.16; H, 4.71; N, 7.60.

\subsubsection{3- $O$-Acetyl-2-azido-4,6- $O$-benzylidene-2-deoxy-D-} glucopyranose (1c). Tetrabutylammonium fluoride in THF $(1.0 \mathrm{M}, 2.50 \mathrm{~mL}, 2.50 \mathrm{mmol})$ was added to a stirred solution of tert-butyldimethylsilyl 3-O-acetyl-2-azido-4,6$O$-benzylidene-2-deoxy- $\beta$-D-glucopyranoside ${ }^{38}(850 \mathrm{mg}$, $1.89 \mathrm{mmol})$ in THF $(10 \mathrm{~mL})-\mathrm{AcOH}(0.16 \mathrm{~mL})$ at $0{ }^{\circ} \mathrm{C}$. After stirring for $15 \mathrm{~min}$, saturated aqueous $\mathrm{NaHCO}_{3}$ (3 $\mathrm{mL}$ ) was added, and the whole was extracted with AcOEt $(50 \mathrm{~mL})$. The organic layer was successively washed with saturated aqueous $\mathrm{NaHCO}_{3}(10 \mathrm{~mL})$ and brine $(2 \times 10 \mathrm{~mL})$, and dried over anhydrous $\mathrm{Na}_{2} \mathrm{SO}_{4}$. Filtration and evaporation in vacuo furnished the crude product $(1.02 \mathrm{~g})$, which was purified by column chromatography (silica gel $30 \mathrm{~g}, 2: 1$ hexane/AcOEt) to give lactol 1c $(621 \mathrm{mg}, 98 \%$, $\alpha: \beta=52: 48)$ as a white amorphous. The anomeric $\alpha: \beta$ ratio of the lactol was determined by ${ }^{1} \mathrm{H}$ NMR. TLC $R_{f}=0.23$ $(2: 1$ hexane/AcOEt $) ;[\alpha]_{\mathrm{D}}{ }^{23}-7.49^{\circ}\left(c 1.02, \mathrm{CHCl}_{3}\right)(\alpha: \beta=$ 52:48); IR (KBr) 3468, 2868, 2112, 1726, 1452, 1371, $1259,1095 \mathrm{~cm}^{-1} ;{ }^{1} \mathrm{H}$ NMR $\left(500 \mathrm{MHz}, \mathrm{CDCl}_{3}\right) \delta 2.14(\mathrm{~s}, 3 \mathrm{H}$, $\mathrm{CH}_{3} \mathrm{CO}$ ), 3.33 (dd, $\left.J=3.6,10.3 \mathrm{~Hz}, 0.5 \mathrm{H}, \mathrm{H}-2 \alpha\right), 3.37$ (br, $0.5 \mathrm{H}, \mathrm{OH}), 3.45(\mathrm{dd}, J=8.0,10.0 \mathrm{~Hz}, 0.5 \mathrm{H}, \mathrm{H}-2 \beta), 3.49$ (ddd, $J=5.0,9.6,10.4 \mathrm{~Hz}, 0.5 \mathrm{H}, \mathrm{H}-5 \beta), 3.63$ (dd, $J=9.5$, $9.6 \mathrm{~Hz}, 1 \mathrm{H}, \mathrm{H}-4 \alpha, \mathrm{H}-4 \beta$ ), 3.73 (dd, $J=10.3,10.4 \mathrm{~Hz}, 0.5 \mathrm{H}$, H-6ax $\alpha$ ), 3.77 (dd, $J=10.4,10.6 \mathrm{~Hz}, 0.5 \mathrm{H}, \mathrm{H}-6 \mathrm{ax} \beta), 3.97$ (br, $0.5 \mathrm{H}, \mathrm{OH}$ ), 4.19 (ddd, $J=5.0,9.5,10.3 \mathrm{~Hz}, 0.5 \mathrm{H}, \mathrm{H}-$ $5 \alpha), 4.28(\mathrm{dd}, J=5.0,10.4 \mathrm{~Hz}, 0.5 \mathrm{H}, \mathrm{H}-6 \mathrm{eq} \alpha), 4.32$ (dd, $J$ $=5.0,10.6 \mathrm{~Hz}, 0.5 \mathrm{H}, \mathrm{H}-6 \mathrm{eq} \beta), 4.77(\mathrm{~d}, J=8.0 \mathrm{~Hz}, 0.5 \mathrm{H}$, $\mathrm{H}-1 \beta$ ), 5.17 (dd, $J=9.5,10.0 \mathrm{~Hz}, 0.5 \mathrm{H}, \mathrm{H}-3 \beta$ ), 5.35 (brd, $J$ $=3.6 \mathrm{~Hz}, 0.5 \mathrm{H}, \mathrm{H}-1 \alpha), 5.48(\mathrm{~s}, 0.5 \mathrm{H}, \mathrm{CHPh}), 5.50(\mathrm{~s}, 0.5 \mathrm{H}$, $\mathrm{CHPh}$ ), 5.64 (dd, $J=9.6,10.3 \mathrm{~Hz}, 0.5 \mathrm{H}, \mathrm{H}-3 \alpha), 7.34-7.38$ (m, 3H, Ar-H), 7.40-7.45 (m, 2H, Ar-H); ${ }^{13} \mathrm{C}$ NMR (126 $\left.\mathrm{MHz} \mathrm{CDCl}_{3}\right) \delta$ 20.8, 62.2, 62.7, 65.7, 66.5, 68.3, 68.8, 69.1, 71.3, 78.6, 79.4, 93.1 (C-1 $\alpha), 96.6(\mathrm{C}-1 \beta), 101.5$, $101.7,126.1,126.2,128.2,129.2,136.6,136.8,170.0$, 170.1; FAB-HRMS $\mathrm{m} / \mathrm{z}$ calcd for $\mathrm{C}_{15} \mathrm{H}_{18} \mathrm{~N}_{3} \mathrm{O}_{6}(\mathrm{M}+\mathrm{H})^{+}$ 336.1196 , found 336.1193 .

\subsubsection{3- $O$-Acetyl-2-azido-4,6- $O$-benzylidene-2-deoxy- $\alpha$ -} D-glucopyranosyl diphenyl phosphate $(2 \mathrm{c})$. The reaction was performed according to the typical procedure $(8 \mathrm{~mL}$ $\left.\mathrm{CH}_{2} \mathrm{Cl}_{2}, 0{ }^{\circ} \mathrm{C}, 0.5 \mathrm{~h}\right)$ employing lactol 1c $(621 \mathrm{mg}, 1.85$ $\mathrm{mmol})$, diphenylphosphoryl chloride $(0.50 \mathrm{~mL}, 2.41 \mathrm{mmol})$, and DMAP (476 mg, $3.90 \mathrm{mmol}$ ). The crude product $(1.14$ g) was purified by column chromatography (silica gel $40 \mathrm{~g}$, 2:1 hexane/AcOEt with $\left.1 \% \quad \mathrm{Et}_{3} \mathrm{~N}\right)$ to give diphenyl phosphates $2 \mathrm{c} \beta$ (714 $\mathrm{mg}, 68 \%$, white solid) and $2 \mathrm{c} \alpha$ (324 $\mathrm{mg}, 31 \%$, colorless syrup). Data for $\alpha$-anomer $(\mathbf{2 c} \alpha)$ : $\mathrm{mp}$ 103.0-105.0 ${ }^{\circ} \mathrm{C}$ (AcOEt-hexane); TLC $R_{f}=0.20 \quad(2: 1$ hexane/AcOEt); $[\alpha]_{\mathrm{D}}^{22}+48.1^{\circ}\left(c 1.50, \mathrm{CHCl}_{3}\right)$; IR (film) 2868, 2114, 1753, 1589, 1489, 1371, 1219, 1186, $954 \mathrm{~cm}^{-1}$; ${ }^{1} \mathrm{H}$ NMR $\left(500 \mathrm{MHz}, \mathrm{CDCl}_{3}\right) \delta 2.14\left(\mathrm{~s}, 3 \mathrm{H}, \mathrm{CH}_{3} \mathrm{CO}\right)$, 3.60-3.66 (m, 3H, H-2, H-4, H-6ax), 3.89-3.95 (m, 2H, H5, H-6eq), 5.45 (s, 1H, CHPh), 5.58 (t, $J=9.9 \mathrm{~Hz}, 1 \mathrm{H}, \mathrm{H}-3)$, $5.99\left(\mathrm{dd}, J=3.5,6.5\left(J_{\mathrm{H}-\mathrm{P}}\right) \mathrm{Hz}, 1 \mathrm{H}, \mathrm{H}-1\right), 7.21(\mathrm{~m}, 2 \mathrm{H}, \mathrm{Ar}-$ $\mathrm{H}), 7.25-7.31(\mathrm{~m}, 4 \mathrm{H}, \mathrm{Ar}-\mathrm{H}), 7.35-7.42$ (m, 9H, Ar-H); ${ }^{13} \mathrm{C}$ NMR $\left(126 \mathrm{MHz}, \mathrm{CDCl}_{3}\right) \delta 20.6,61.7\left(\mathrm{~d}, J_{\mathrm{C}-\mathrm{P}}=8.9 \mathrm{~Hz}\right)$, 64.6, 68.0, 68.9, 78.4, 96.9 (d, $\left.J_{\mathrm{C}-\mathrm{P}}=5.5 \mathrm{~Hz}, \mathrm{C}-1\right), 101.1$, $119.9\left(\mathrm{~d}, J_{\mathrm{C}-\mathrm{P}}=5.0 \mathrm{~Hz}\right), 120.2\left(\mathrm{~d}, J_{\mathrm{C}-\mathrm{P}}=5.0 \mathrm{~Hz}\right), 125.6$, $126.0,128.1,129.1,129.7,129.8,136.5,150.1,\left(\mathrm{~d}, J_{\mathrm{C}-\mathrm{P}}=\right.$ $5.0 \mathrm{~Hz}), 150.2\left(\mathrm{~d}, J_{\mathrm{C}-\mathrm{P}}=5.0 \mathrm{~Hz}\right), 169.4 ;{ }^{31} \mathrm{P}$ NMR $(109$ $\left.\mathrm{MHz}, \quad \mathrm{CDCl}_{3}\right) \quad \delta-13.0 ;$ FAB-HRMS $\mathrm{m} / \mathrm{z}$ calcd for $\mathrm{C}_{27} \mathrm{H}_{27} \mathrm{~N}_{3} \mathrm{O}_{9} \mathrm{P}(\mathrm{M}+\mathrm{H})^{+}$568.1485, found 568.1467. Data for $\beta$-anomer (2c $\beta$ ): TLC $R_{f}=0.39$ (2:1 hexane/AcOEt); $[\alpha]_{\mathrm{D}}{ }^{22}$ $-52.8^{\circ}$ (c 1.50, $\mathrm{CHCl}_{3}$ ); IR (film) 2868, 2114, 1755, 1589, 1489, 1371, 1219, 1186, $958 \mathrm{~cm}^{-1}$; ${ }^{1} \mathrm{H}$ NMR $(500 \mathrm{MHz}$, $\left.\mathrm{CDCl}_{3}\right) \delta 2.13\left(\mathrm{~s}, 3 \mathrm{H}, \mathrm{CH}_{3} \mathrm{CO}\right), 3.56$ (ddd, $J=4.9,9.6,10.2$ $\mathrm{Hz}, 1 \mathrm{H}, \mathrm{H}-5), 3.63$ (m, 1H, H-2), 3.65 (dd, $J=9.6,10.3 \mathrm{~Hz}$, $1 \mathrm{H}, \mathrm{H}-4), 3.67$ (dd, $J=10.2,10.4 \mathrm{~Hz}, 1 \mathrm{H}, \mathrm{H}-6 \mathrm{ax}), 4.23$ (dd, $J=4.9,10.4 \mathrm{~Hz}, 1 \mathrm{H}, \mathrm{H}-6 \mathrm{eq}), 5.22(\mathrm{dd}, J=8.9,10.3 \mathrm{~Hz}, 1 \mathrm{H}$, $\mathrm{H}-3), 5.31$ (dd, $\left.J=7.7,7.9\left(J_{\mathrm{H}-\mathrm{P}}\right) \mathrm{Hz}, 1 \mathrm{H}, \mathrm{H}-1\right), 5.46$ (s, $1 \mathrm{H}$, $\mathrm{CHPh}$, 7.20-7.27 (m, 6H, Ar-H), 7.34-7.41 (m, 9H, Ar$\mathrm{H}) ;{ }^{13} \mathrm{C}$ NMR $\left(126 \mathrm{MHz}, \mathrm{CDCl}_{3}\right) \delta 20.5,64.7$ (d, $J_{\mathrm{C}-\mathrm{P}}=8.8$ $\mathrm{Hz}), 66.8,67.7,71.1\left(\mathrm{~d}, J_{\mathrm{C}-\mathrm{P}}=1.1 \mathrm{~Hz}\right), 77.7,98.0\left(\mathrm{~d}, J_{\mathrm{C}-\mathrm{P}}=\right.$ $5.0 \mathrm{~Hz}, \mathrm{C}-1), 101.4,119.8\left(\mathrm{~d}, J_{\mathrm{C}-\mathrm{P}}=5.0 \mathrm{~Hz}\right), 120.1\left(\mathrm{~d}, J_{\mathrm{C}-\mathrm{P}}\right.$ $=5.0 \mathrm{~Hz}), 125.5,125.6,125.9,128.1,129.0,129.6,129.7$, $136.4,150.05\left(\mathrm{~d}, J_{\mathrm{C}-\mathrm{P}}=6.3 \mathrm{~Hz}\right), 150.11\left(\mathrm{~d}, J_{\mathrm{C}-\mathrm{P}}=6.3 \mathrm{~Hz}\right)$, 169.2; ${ }^{31} \mathrm{P}$ NMR $\left(109 \mathrm{MHz}, \mathrm{CDCl}_{3}\right) \delta-13.8$; FAB-HRMS $m / z$ calcd for $\mathrm{C}_{27} \mathrm{H}_{27} \mathrm{~N}_{3} \mathrm{O}_{9} \mathrm{P} \quad(\mathrm{M}+\mathrm{H})^{+}$568.1485, found 568.1468; Anal. calcd for $\mathrm{C}_{27} \mathrm{H}_{26} \mathrm{~N}_{3} \mathrm{O}_{9} \mathrm{P}$ : C, 57.15; H, 4.62; $\mathrm{N}, 7.40$, found $\mathrm{C}, 57.22 ; \mathrm{H}, 4.61 ; \mathrm{N}, 7.49$. 
4.1.5. 2-Azido-3,4,6-tri- $O$-benzyl-2-deoxy- $\alpha$-D-galactopyranosyl diphenyl phosphate (4a). The reaction was performed according to the typical procedure $(10 \mathrm{~mL}$ $\left.\mathrm{CH}_{2} \mathrm{Cl}_{2}, 0{ }^{\circ} \mathrm{C}, 0.5 \mathrm{~h}\right)$ with lactol $3 \mathrm{a}^{7 \mathrm{a}}(1.10 \mathrm{~g}, 2.31 \mathrm{mmol})$, diphenylphosphoryl chloride $(0.63 \mathrm{~mL}, 3.02 \mathrm{mmol})$, and DMAP (567 mg, $4.63 \mathrm{mmol})$. The crude product $(1.68 \mathrm{~g}$ ) was purified by column chromatography (silica gel $40 \mathrm{~g}$, 3:1 hexane/AcOEt with $\left.2 \% \quad \mathrm{Et}_{3} \mathrm{~N}\right)$ to give diphenyl phosphate 4a $(1.30 \mathrm{~g}, 79 \%, \alpha: \beta=58: 42)$ as a colorless syrup. TLC $R_{f}=0.45(\alpha), 0.31(\beta)(2: 1$ hexane/AcOEt); $[\alpha]_{\mathrm{D}}^{22}+55.3^{\circ}\left(c 1.50, \mathrm{CHCl}_{3}\right)(\alpha: \beta=90: 10)$; IR (film) 3032 , 2872, 2114, 1591, 1489, 1290, 1188, $958 \mathrm{~cm}^{-1}$; ${ }^{1} \mathrm{H}$ NMR $\left(500 \mathrm{MHz}, \mathrm{CDCl}_{3}\right) \delta 3.28(\mathrm{dd}, J=5.4,9.0 \mathrm{~Hz}, 0.6 \mathrm{H}, \mathrm{H}-$ $6 \mathrm{a} \alpha), 3.39(\mathrm{dd}, J=2.7,10.4 \mathrm{~Hz}, 0.4 \mathrm{H}, \mathrm{H}-3 \beta), 3.45(\mathrm{dd}, J=$ $4.7,8.5 \mathrm{~Hz}, 0.4 \mathrm{H}, \mathrm{H}-6 \mathrm{a} \beta), 3.56(\mathrm{dd}, J=8.1,9.0 \mathrm{~Hz}, 0.6 \mathrm{H}$, $\mathrm{H}-6 \mathrm{~b} \alpha$ ), 3.58 (dd, $J=8.0,8.5 \mathrm{~Hz}, 0.4 \mathrm{H}, \mathrm{H}-6 \mathrm{~b} \beta$ ), 3.61 (dd, $J$ $=4.7,8.0 \mathrm{~Hz}, 0.4 \mathrm{H}, \mathrm{H}-5 \beta), 3.86(\mathrm{dd}, J=2.5,10.5 \mathrm{~Hz}, 0.6 \mathrm{H}$, $\mathrm{H}-3 \alpha), 3.90(\mathrm{dd}, J=8.0,10.4 \mathrm{~Hz}, 0.4 \mathrm{H}, \mathrm{H}-2 \beta), 3.92$ (d, $J=$ $2.7 \mathrm{~Hz}, 0.4 \mathrm{H}, \mathrm{H}-4 \beta$ ), 4.01 (dd, $J=5.4,8.1 \mathrm{~Hz}, 0.6 \mathrm{H}, \mathrm{H}-5 \alpha$ ), 4.05 (brs, $0.6 \mathrm{H}, \mathrm{H}-4 \alpha), 4.10$ (ddd, $J=3.3,10.5,3.2\left(J_{\mathrm{H}-\mathrm{P}}\right)$ $\mathrm{Hz}, 0.6 \mathrm{H}, \mathrm{H}-2 \alpha), 4.36$ (d, $J=12.6 \mathrm{~Hz}, 0.6 \mathrm{H}, \mathrm{OCHPh}), 4.38$ $(\mathrm{d}, J=12.6 \mathrm{~Hz}, 0.6 \mathrm{H}, \mathrm{OCHPh}), 4.39$ (d, $J=11.6 \mathrm{~Hz}, 0.4 \mathrm{H}$, $\mathrm{OCHPh}), 4.41(\mathrm{~d}, J=11.6 \mathrm{~Hz}, 0.4 \mathrm{H}, \mathrm{OCHPh}), 4.52(\mathrm{~d}, J=$ $11.2 \mathrm{~Hz}, 0.6 \mathrm{H}, \mathrm{OCHPh}), 4.55(\mathrm{~d}, J=11.4 \mathrm{~Hz}, 0.4 \mathrm{H}$, OCHPh), $4.64(\mathrm{~d}, J=11.7 \mathrm{~Hz}, 0.4 \mathrm{H}, \mathrm{OCHPh}), 4.65(\mathrm{~d}, J=$ $11.4 \mathrm{~Hz}, 0.6 \mathrm{H}, \mathrm{OCHPh}), 4.69(\mathrm{~d}, J=11.7 \mathrm{~Hz}, 0.4 \mathrm{H}$, OCHPh), $4.71(\mathrm{~d}, J=11.4 \mathrm{~Hz}, 0.6 \mathrm{H}, \mathrm{OC} H \mathrm{Ph}), 4.85(\mathrm{~d}, J=$ $11.2 \mathrm{~Hz}, 0.6 \mathrm{H}, \mathrm{OCHPh}), 4.87(\mathrm{~d}, J=11.4 \mathrm{~Hz}, 0.4 \mathrm{H}$, OCHPh), $5.09\left(\mathrm{dd}, J=8.0,7.2\left(J_{\mathrm{H}-\mathrm{P}}\right) \mathrm{Hz}, 0.4 \mathrm{H}, \mathrm{H}-1 \beta\right), 5.94$ $\left(\mathrm{dd}, J=3.3,5.7\left(J_{\mathrm{H}-\mathrm{P}}\right) \mathrm{Hz}, 0.6 \mathrm{H}, \mathrm{H}-1 \alpha\right), 7.11-7.39(\mathrm{~m}, 25 \mathrm{H}$, $\mathrm{Ar}-\mathrm{H}) ;{ }^{13} \mathrm{C}$ NMR $\left(126 \mathrm{MHz}, \mathrm{CDCl}_{3}\right) \delta 59.4\left(\mathrm{~d}, J_{\mathrm{C}-\mathrm{P}}=8.4\right.$ $\mathrm{Hz}), 63.1\left(\mathrm{~d}, J_{\mathrm{C}-\mathrm{P}}=9.1 \mathrm{~Hz}\right), 67.4,67.5,71.5,72.0,72.4$, $72.5,73.30,73.34,74.2,74.6,74.8,77.0,77.2,80.4\left(\mathrm{~d}, J_{\mathrm{C}-\mathrm{P}}\right.$ $=2.4 \mathrm{~Hz}), 97.8\left(\mathrm{~d}, J_{\mathrm{C}-\mathrm{P}}=5.9 \mathrm{~Hz}, \mathrm{C}-1 \alpha\right), 98.3\left(\mathrm{~d}, J_{\mathrm{C}-\mathrm{P}}=5.2\right.$ $\mathrm{Hz}, \mathrm{C}-1 \beta), 119.9\left(\mathrm{~d}, J_{\mathrm{C}-\mathrm{P}}=5.0 \mathrm{~Hz}\right), 120.0\left(\mathrm{~d}, J_{\mathrm{C}-\mathrm{P}}=5.0 \mathrm{~Hz}\right)$, $120.1\left(\mathrm{~d}, J_{\mathrm{C}-\mathrm{P}}=5.0 \mathrm{~Hz}\right), 120.4\left(\mathrm{~d}, J_{\mathrm{C}-\mathrm{P}}=5.0 \mathrm{~Hz}\right), 125.2$, $125.3,125.4,125.5,127.60,127.64,127.67,127.73,127.8$, $127.86,127.89,128.15,128.18,128.3,128.4,129.4,129.5$, $129.6,129.7,137.08,137.12,137.5,137.9,138.0,150.2(\mathrm{~d}$, $\left.J_{\text {C-P }}=7.5 \mathrm{~Hz}\right), 150.30\left(\mathrm{~d}, J_{\mathrm{C}-\mathrm{P}}=7.5 \mathrm{~Hz}\right), 150.31\left(\mathrm{~d}, J_{\mathrm{C}-\mathrm{P}}=\right.$ $7.5 \mathrm{~Hz}), 150.36\left(\mathrm{~d}, J_{\mathrm{C}-\mathrm{P}}=7.5 \mathrm{~Hz}\right) ;{ }^{31} \mathrm{P}$ NMR $(109 \mathrm{MHz}$, $\left.\mathrm{CDCl}_{3}\right) \delta-13.32(\beta),-13.25(\alpha)$; FAB-HRMS $\mathrm{m} / \mathrm{z}$ calcd for $\mathrm{C}_{39} \mathrm{H}_{39} \mathrm{~N}_{3} \mathrm{O}_{8} \mathrm{P}(\mathrm{M}+\mathrm{H})^{+}$708.2474, found 708.2451; Anal. calcd for $\mathrm{C}_{39} \mathrm{H}_{38} \mathrm{~N}_{3} \mathrm{O}_{8} \mathrm{P}: \mathrm{C}, 66.19 ; \mathrm{H}, 5.41 ; \mathrm{N}, 5.94$, found $\mathrm{C}$, 66.35; H, 5.59; N, 5.85.

4.1.6. 3,4,6-Tri- $O$-acetyl-2-azido-2-deoxy-D-galactopyranosyl diphenyl phosphate (4b). The reaction was performed according to the typical procedure $\left(10 \mathrm{~mL} \mathrm{CH}_{2} \mathrm{Cl}_{2}\right.$, $\left.0{ }^{\circ} \mathrm{C}, 0.5 \mathrm{~h}\right)$ with lactol $\mathbf{3 b}^{36}(994 \mathrm{mg}, 3.00 \mathrm{mmol})$, diphenylphosphoryl chloride $(0.81 \mathrm{~mL}, 3.90 \mathrm{mmol})$, and DMAP (953 mg, $7.80 \mathrm{mmol})$. The crude product $(1.96 \mathrm{~g}$ ) was purified by column chromatography (silica gel $40 \mathrm{~g}$, 2:1 hexane/AcOEt) to give diphenyl phosphate $4 \mathbf{b}(1.64 \mathrm{~g}$, $97 \%, \alpha: \beta=24: 76)$ as a colorless syrup. Data for $\alpha$-anomer (4b $\alpha)$ : TLC $R_{f}=0.58\left(10: 1 \mathrm{CH}_{2} \mathrm{Cl}_{2} /\right.$ acetone $) ;[\alpha]_{\mathrm{D}}{ }^{24}+74.2^{\circ}$ (c 1.50, $\mathrm{CHCl}_{3}$ ); IR (film) 2116, 1753, 1591, 1489, 1371, 1226, $960 \mathrm{~cm}^{-1} ;{ }^{1} \mathrm{H}$ NMR $\left(500 \mathrm{MHz}, \mathrm{CDCl}_{3}\right) \delta 1.90(\mathrm{~s}, 3 \mathrm{H}$, $\mathrm{CH}_{3} \mathrm{CO}$ ), 2.07 (s, 3H, $\mathrm{CH}_{3} \mathrm{CO}$ ), 2.14 (s, 3H, $\mathrm{CH}_{3} \mathrm{CO}$ ), 3.86 (dd, $J=6.4,11.3 \mathrm{~Hz}, 1 \mathrm{H}, \mathrm{H}-6 \mathrm{a}), 3.96$ (ddd, $J=3.3,11.0$, $\left.3.2\left(J_{\mathrm{H}-\mathrm{P}}\right) \mathrm{Hz}, 1 \mathrm{H}, \mathrm{H}-2\right), 4.06(\mathrm{dd}, J=6.8,11.3 \mathrm{~Hz}, 1 \mathrm{H}, \mathrm{H}-$ 6b), 4.29 (dd, $J=6.4,6.8 \mathrm{~Hz}, 1 \mathrm{H}, \mathrm{H}-5), 5.30(\mathrm{dd}, J=3.2$, $11.0 \mathrm{~Hz}, 1 \mathrm{H}, \mathrm{H}-3$ ), 5.46 (brd, $J=3.2 \mathrm{~Hz}, 1 \mathrm{H}, \mathrm{H}-4$ ), 6.04 $\left(\mathrm{dd}, J=3.3,6.1\left(J_{\mathrm{H}-\mathrm{P}}\right) \mathrm{Hz}, 1 \mathrm{H}, \mathrm{H}-1\right), 7.21(\mathrm{~m}, 2 \mathrm{H}, \mathrm{Ar}-\mathrm{H})$, 7.25-7.28 (m, 4H, Ar-H), 7.34-7.38 (m, 4H, Ar-H); ${ }^{13} \mathrm{C}$ NMR $\left(126 \mathrm{MHz}, \mathrm{CDCl}_{3}\right) \delta 20.4,20.47,20.50,57.4\left(\mathrm{~d}, J_{\mathrm{C}-\mathrm{P}}\right.$ $=8.7 \mathrm{~Hz}), 60.8,66.7,68.4,68.7,96.7\left(\mathrm{~d}, J_{\mathrm{C}-\mathrm{P}}=5.5 \mathrm{~Hz}, \mathrm{C}-\right.$ $1), 120.0\left(\mathrm{~d}, J_{\mathrm{C}-\mathrm{P}}=5.0 \mathrm{~Hz}\right), 120.2\left(\mathrm{~d}, J_{\mathrm{C}-\mathrm{P}}=5.0 \mathrm{~Hz}\right), 125.60$, $125.63,129.7,129.8,150.2\left(\mathrm{~d}, J_{\mathrm{C}-\mathrm{P}}=6.3 \mathrm{~Hz}\right), 150.3\left(\mathrm{~d}, J_{\mathrm{C}-\mathrm{P}}\right.$ $=6.3 \mathrm{~Hz}), 169.6,169.8,170.1 ;{ }^{31} \mathrm{P}$ NMR $(109 \mathrm{MHz}$, $\left.\mathrm{CDCl}_{3}\right) \delta-13.1$; FAB-HRMS $\mathrm{m} / z$ calcd for $\mathrm{C}_{24} \mathrm{H}_{27} \mathrm{~N}_{3} \mathrm{O}_{11} \mathrm{P}$ $(\mathrm{M}+\mathrm{H})^{+}$564.1383, found 564.1368. Data for $\beta$-anomer $(4 \mathrm{~b} \beta):$ TLC $R_{f}=0.42\left(10: 1 \mathrm{CH}_{2} \mathrm{Cl}_{2}\right.$ /acetone $) ;[\alpha]_{\mathrm{D}}{ }^{24}+5.60^{\circ}$ (c 1.50, $\mathrm{CHCl}_{3}$ ); IR (film) 2116, 1753, 1591, 1489, 1371, 1226, $960 \mathrm{~cm}^{-1}$; ${ }^{1} \mathrm{H}$ NMR $\left(500 \mathrm{MHz}, \mathrm{CDCl}_{3}\right) \delta 1.98$ (s, 3H, $\mathrm{CH}_{3} \mathrm{CO}$ ), 2.05 (s, 3H, CH $\mathrm{CO}$ ), 2.16 (s, 3H, $\left.\mathrm{CH}_{3} \mathrm{CO}\right), 3.82$ $(\mathrm{dd}, J=8.2,10.7 \mathrm{~Hz}, 1 \mathrm{H}, \mathrm{H}-2), 3.97(\mathrm{dt}, J=0.6,6.5 \mathrm{~Hz}$, $1 \mathrm{H}, \mathrm{H}-5), 4.02$ (dd, $J=6.5,11.0 \mathrm{~Hz}, 1 \mathrm{H}, \mathrm{H}-6 \mathrm{a}), 4.10$ (dd, $J$ $=6.5,11.0 \mathrm{~Hz}, 1 \mathrm{H}, \mathrm{H}-6 \mathrm{~b}), 4.87(\mathrm{dd}, J=3.3,10.7 \mathrm{~Hz}, 1 \mathrm{H}$, $\mathrm{H}-3), 5.24$ (dd, $\left.J=8.2,7.4\left(J_{\mathrm{H}-\mathrm{P}}\right) \mathrm{Hz}, 1 \mathrm{H}, \mathrm{H}-1\right), 5.36$ (dd, $J$ $=0.6,3.3 \mathrm{~Hz}, 1 \mathrm{H}, \mathrm{H}-4), 7.22(\mathrm{~m}, 2 \mathrm{H}, \mathrm{Ar}-\mathrm{H}), 7.26-7.28(\mathrm{~m}$, $4 \mathrm{H}, \mathrm{Ar}-\mathrm{H}), 7.34-7.37$ (m, 4H, Ar-H); ${ }^{13} \mathrm{C}$ NMR (126 MHz, $\left.\mathrm{CDCl}_{3}\right) \delta 20.4,20.5,60.7,61.0\left(\mathrm{~d}, J_{\mathrm{C}-\mathrm{P}}=9.4 \mathrm{~Hz}\right), 65.9$, $71.2\left(\mathrm{~d}, J_{\mathrm{C}-\mathrm{P}}=1.6 \mathrm{~Hz}\right), 71.8,98.1\left(\mathrm{~d}, J_{\mathrm{C}-\mathrm{P}}=5.3 \mathrm{~Hz}, \mathrm{C}-1\right)$, $120.0\left(\mathrm{~d}, J_{\mathrm{C}-\mathrm{P}}=3.4 \mathrm{~Hz}\right), 120.3\left(\mathrm{~d}, J_{\mathrm{C}-\mathrm{P}}=3.4 \mathrm{~Hz}\right), 125.6$, $125.7,129.7,129.8,150.2\left(\mathrm{~d}, J_{\mathrm{C}-\mathrm{P}}=8.8 \mathrm{~Hz}\right), 150.3\left(\mathrm{~d}, J_{\mathrm{C}-\mathrm{P}}\right.$ $=8.8 \mathrm{~Hz}), 169.5,169.8,170.2 ;{ }^{31} \mathrm{P}$ NMR $(109 \mathrm{MHz}$, $\left.\mathrm{CDCl}_{3}\right) \delta-13.5$; FAB-HRMS $\mathrm{m} / z$ calcd for $\mathrm{C}_{24} \mathrm{H}_{27} \mathrm{~N}_{3} \mathrm{O}_{11} \mathrm{P}$ $(\mathrm{M}+\mathrm{H})^{+}$564.1383, found 564.1385; Anal. calcd for: $\mathrm{C}_{24} \mathrm{H}_{26} \mathrm{~N}_{3} \mathrm{O}_{11} \mathrm{P}: \mathrm{C}, 51.16 ; \mathrm{H}, 4.65 ; \mathrm{N}, 7.46$, found $\mathrm{C}$, 51.02; $\mathrm{H}, 4.72 ; \mathrm{N}, 7.47$.

\subsubsection{3- $O$-Acetyl-2-azido-4,6- $O$-benzylidene-2-deoxy- $\alpha$ -} D-galactopyranosyl diphenyl phosphate $(4 \mathrm{c})$. The reaction was performed according to the typical procedure (6 $\mathrm{mL} \mathrm{CH}_{2} \mathrm{Cl}_{2}, 0{ }^{\circ} \mathrm{C}, 0.5 \mathrm{~h}$ ) employing lactol $3 \mathrm{c}^{37}$ (350 mg, $1.04 \mathrm{mmol})$, diphenylphosphoryl chloride $(0.28 \mathrm{~mL}, 1.36$ $\mathrm{mmol})$, and DMAP (254 $\mathrm{mg}, 2.08 \mathrm{mmol})$. The crude product $(530 \mathrm{mg}$ ) was purified by column chromatography (silica gel $25 \mathrm{~g}, 2: 1 \rightarrow 1: 1$ hexane/AcOEt with $1 \% \mathrm{Et}_{3} \mathrm{~N}$ ) to give diphenyl phosphates $\mathbf{4} \boldsymbol{c} \alpha$ (295 $\mathrm{mg}, 50 \%)$ and $\mathbf{4 c} \beta$ (147 $\mathrm{mg}, 25 \%)$ as white amorphous. Data for $\alpha$-anomer $(\mathbf{4 c} \alpha)$ : TLC $R_{f}=0.44(1: 1$ hexane/AcOEt $) ;[\alpha]_{\mathrm{D}}{ }^{24}+152.5^{\circ}(c 1.50$, $\mathrm{CHCl}_{3}$ ); IR (KBr) 3069, 2922, 2116, 1747, 1591, 1489, 1224, 1188, 958, $756 \mathrm{~cm}^{-1} ;{ }^{1} \mathrm{H}$ NMR $\left(500 \mathrm{MHz}, \mathrm{CDCl}_{3}\right) \delta$ 2.14 (s, 3H, CH $\mathrm{CO}$ ), 3.66 (brs, $1 \mathrm{H}, \mathrm{H}-5), 3.83$ (dd, $J=1.0$, $13.0 \mathrm{~Hz}, 1 \mathrm{H}, \mathrm{H}-6 \mathrm{a}), 3.95$ (dd, $J=0.9,13.0 \mathrm{~Hz}, 1 \mathrm{H}, \mathrm{H}-6 \mathrm{~b}$ ), 4.20 (ddd, $J=3.2$, 11.0, $\left.3.2\left(J_{\mathrm{H}-\mathrm{P}}\right) \mathrm{Hz}, 1 \mathrm{H}, \mathrm{H}-2\right), 4.42$ (brd, $J=5.4 \mathrm{~Hz}, 1 \mathrm{H}, \mathrm{H}-4), 5.23(\mathrm{dd}, J=3.3,11.0 \mathrm{~Hz}, 1 \mathrm{H}, \mathrm{H}-3)$, $5.46(\mathrm{~s}, 1 \mathrm{H}, \mathrm{CHPh}), 6.10\left(\mathrm{dd}, J=3.2,6.0\left(J_{\mathrm{H}-\mathrm{P}}\right) \mathrm{Hz}, 1 \mathrm{H}, \mathrm{H}-\right.$ 1), 7.18 (m, 2H, Ar-H), 7.25-7.38 (m, 11H, Ar-H), 7.46 (m, $2 \mathrm{H}, \mathrm{Ar}-\mathrm{H}) ;{ }^{13} \mathrm{C}$ NMR $\left(126 \mathrm{MHz}, \mathrm{CDCl}_{3}\right) \delta 20.8,57.0(\mathrm{~d}$, $\left.J_{\mathrm{C}-\mathrm{P}}=8.6 \mathrm{~Hz}\right), 64.3,68.3,69.4,72.6,97.6\left(\mathrm{~d}, J_{\mathrm{C}-\mathrm{P}}=5.0 \mathrm{~Hz}\right.$, C-1), 100.6, 120.0 (d, $\left.J_{\mathrm{C}-\mathrm{P}}=5.0 \mathrm{~Hz}\right), 120.2\left(\mathrm{~d}, J_{\mathrm{C}-\mathrm{P}}=5.0\right.$ $\mathrm{Hz}), 125.4,125.5,126.0,128.1,129.1,129.67,129.72$, $137.1,150.2\left(\mathrm{~d}, J_{\mathrm{C}-\mathrm{P}}=4.4 \mathrm{~Hz}\right), 150.3\left(\mathrm{~d}, J_{\mathrm{C}-\mathrm{P}}=4.4 \mathrm{~Hz}\right)$, 170.2; ${ }^{31} \mathrm{P}$ NMR (202 MHz, $\mathrm{CDCl}_{3}$ ) $\delta-12.9$; FAB-HRMS $m / z$ calcd for $\mathrm{C}_{27} \mathrm{H}_{27} \mathrm{~N}_{3} \mathrm{O}_{9} \mathrm{P} \quad(\mathrm{M}+\mathrm{H})^{+}$568.1485, found 568.1501; Anal. calcd for: $\mathrm{C}_{27} \mathrm{H}_{26} \mathrm{~N}_{3} \mathrm{O}_{9} \mathrm{P}$ : C, 57.15; H, 4.62; $\mathrm{N}, 7.40$, found $\mathrm{C}, 57.20 ; \mathrm{H}, 4.64 ; \mathrm{N}, 7.39$. Data for $\beta$ anomer $(\mathbf{4 c} \beta)$ : TLC $R_{f}=0.28(1: 1$ hexane/AcOEt $) ;[\alpha]_{\mathrm{D}}{ }^{26}$ $+73.8^{\circ}$ (c 1.50, $\mathrm{CHCl}_{3}$ ); IR (KBr) 3069, 2905, 2118, 1749, 
1591, 1491, 1371, 1294, 1186, $1087 \mathrm{~cm}^{-1} ;{ }^{1} \mathrm{H}$ NMR (500 $\mathrm{MHz}, \mathrm{CDCl}_{3}$ ) $\delta 2.12$ (s, 3H, $\mathrm{CH}_{3} \mathrm{CO}$ ), 3.56 (brs, $1 \mathrm{H}, \mathrm{H}-5$ ), $3.95(\mathrm{dd}, J=1.3,12.5 \mathrm{~Hz}, 1 \mathrm{H}, \mathrm{H}-6 \mathrm{a}), 4.00(\mathrm{dd}, J=8.2$, $10.8 \mathrm{~Hz}, 1 \mathrm{H}, \mathrm{H}-2), 4.17$ (dd, $J=1.2,12.5 \mathrm{~Hz}, 1 \mathrm{H}, \mathrm{H}-6 \mathrm{~b}$ ), $4.34(\mathrm{~d}, J=3.3 \mathrm{~Hz}, 1 \mathrm{H}, \mathrm{H}-4), 4.78(\mathrm{dd}, J=3.3,10.8 \mathrm{~Hz}$, $1 \mathrm{H}, \mathrm{H}-3), 5.25\left(\mathrm{dd}, J=8.2,6.7\left(J_{\mathrm{H}-\mathrm{P}}\right) \mathrm{Hz}, 1 \mathrm{H}, \mathrm{H}-3\right), 5.49$ (s, 1H, CHPh), 7.18 (m, 2H, Ar-H), 7.24-7.43 (m, 11H, Ar-H), $7.51(\mathrm{~m}, 2 \mathrm{H}, \mathrm{Ar}-\mathrm{H}) ;{ }^{13} \mathrm{C}$ NMR $\left(126 \mathrm{MHz}, \mathrm{CDCl}_{3}\right) \delta 20.8$, $60.5\left(\mathrm{~d}, J_{\mathrm{C}-\mathrm{P}}=10.1 \mathrm{~Hz}\right), 67.0,68.3,72.0,72.2\left(\mathrm{~d}, J_{\mathrm{C}-\mathrm{P}}=1.5\right.$ $\mathrm{Hz}), 98.2\left(\mathrm{~d}, J_{\mathrm{C}-\mathrm{P}}=5.0 \mathrm{~Hz}, \mathrm{C}-1\right), 100.8,120.0\left(\mathrm{~d}, J_{\mathrm{C}-\mathrm{P}}=5.0\right.$ $\mathrm{Hz}), 120.7\left(\mathrm{~d}, J_{\mathrm{C}-\mathrm{P}}=5.0 \mathrm{~Hz}\right), 125.5,126.2,128.2,129.2$, $129.6,129.7,137.4,150.2\left(\mathrm{~d}, J_{\mathrm{C}-\mathrm{P}}=6.3 \mathrm{~Hz}\right), 150.3\left(\mathrm{~d}, J_{\mathrm{C}-\mathrm{P}}\right.$ $=7.5 \mathrm{~Hz}), 170.1 ;{ }^{31} \mathrm{P}$ NMR $\left(202 \mathrm{MHz}, \mathrm{CDCl}_{3}\right) \delta-13.1$; FAB-HRMS $m / z$ calcd for $\mathrm{C}_{27} \mathrm{H}_{27} \mathrm{~N}_{3} \mathrm{O}_{9} \mathrm{P}(\mathrm{M}+\mathrm{H})^{+}$568.1485, found 568.1470 .

\subsubsection{2-Azido-3,4,6-tri- $O$-benzyl-2-deoxy-D-glucopyra-} nosyl diphenyl phosphate (2a) $(\alpha: \beta=2: 98)$. Diphenyl phosphate $(207 \mathrm{mg}, 0.83 \mathrm{mmol})$ was added to a stirred solution of $\mathbf{5} \alpha^{7 \mathrm{~b}}(514 \mathrm{mg}, 0.83 \mathrm{mmol})$ in $\mathrm{CH}_{2} \mathrm{Cl}_{2}(7 \mathrm{~mL})$ at $0{ }^{\circ} \mathrm{C}$. After $0.1 \mathrm{~h}$, the mixture was poured into a two-layer mixture of $\mathrm{Et}_{2} \mathrm{O}(5 \mathrm{~mL})$ and saturated aqueous $\mathrm{NaHCO}_{3}(5$ $\mathrm{mL}$ ), and the whole was extracted with AcOEt (30 mL). The organic layer was successively washed with saturated aqueous $\mathrm{NaHCO}_{3}(10 \mathrm{~mL})$ and brine $(2 \times 10 \mathrm{~mL})$, and dried over anhydrous $\mathrm{Na}_{2} \mathrm{SO}_{4}$. Filtration and evaporation in vacuo furnished the pale yellow oil $(735 \mathrm{mg})$, which was purified by column chromatography (silica gel $15 \mathrm{~g}, 2: 1$ hexane/AcOEt with $2 \% \mathrm{Et}_{3} \mathrm{~N}$ ) to give diphenyl phosphate 2a $(507 \mathrm{mg}, 86 \%, \alpha: \beta=2: 98)$ as a colorless oil. The anomeric $\alpha: \beta$ ratio of the product was determined by ${ }^{31} \mathrm{P}$ NMR.

\subsubsection{2-Azido-3,4,6-tri- $O$-benzyl-2-deoxy-D-glucopyra-} nosyl $N, N, N$ ', $N$ '-tetramethylphosphorodiamidate (6). Butyllithium in hexane $(1.56 \mathrm{M}, 0.3 \mathrm{~mL}, 0.468 \mathrm{mmol})$ was added to a stirred solution of $\mathbf{1 a}(212 \mathrm{mg}, 0.446 \mathrm{mmol})$ in THF $(5.0 \mathrm{~mL})$ at $-78{ }^{\circ} \mathrm{C}$. After $15 \mathrm{~min}$, a solution of bis(dimethylamino)phosphoryl chloride $(0.067 \mathrm{~mL}, 0.450$ mmol) in HMPA $(0.5 \mathrm{~mL})$ was added, and the mixture was allowed to warm to $-20{ }^{\circ} \mathrm{C}$ over $30 \mathrm{~min}$. After stirring at this temperature for $2 \mathrm{~h}$, the reaction was quenched with crushed ice, followed by stirring at $0{ }^{\circ} \mathrm{C}$ for $30 \mathrm{~min}$. The mixture was poured into a two-layer mixture of $\mathrm{Et}_{2} \mathrm{O}(5$ $\mathrm{mL})$ and saturated aqueous $\mathrm{NaHCO}_{3}(5 \mathrm{~mL})$, and the whole was extracted with AcOEt $(20 \mathrm{~mL})$. The organic layer was washed with brine $(2 \times 10 \mathrm{~mL})$, and dried over anhydrous $\mathrm{Na}_{2} \mathrm{SO}_{4}$. Filtration and evaporation in vacuo furnished the yellow residue $(301 \mathrm{mg}$ ), which was purified by column chromatography (silica gel $8 \mathrm{~g}, 1: 1 \rightarrow 1: 2$ hexane/AcOEt) to give diamidate $6(238 \mathrm{mg}, 87 \%, \alpha: \beta=67: 33)$ as a colorless oil. The anomeric $\alpha: \beta$ ratio of the product was determined by ${ }^{31} \mathrm{P}$ NMR. TLC $R_{f}=0.31(\mathrm{AcOEt}) ;[\alpha]_{\mathrm{D}}{ }^{22}$ $+13.9^{\circ}\left(c\right.$ 1.27, $\left.\mathrm{CHCl}_{3}\right)(\alpha: \beta=67: 33)$; IR $\left(\mathrm{CHCl}_{3}\right) 3034$, 2932, 2114, 1454, 1305, 1215, $995 \mathrm{~cm}^{-1} ;{ }^{1} \mathrm{H}$ NMR (500 $\left.\mathrm{MHz}, \mathrm{CDCl}_{3}\right) \delta 2.62\left(\mathrm{~d}, J_{\mathrm{H}-\mathrm{P}}=10.1 \mathrm{~Hz}, 4.2 \mathrm{H}, \mathrm{N}\left(\mathrm{CH}_{3}\right)_{2}\right)$, $2.63\left(\mathrm{~d}, J_{\mathrm{H}-\mathrm{P}}=10.4 \mathrm{~Hz}, 1.8 \mathrm{H}, \mathrm{N}\left(\mathrm{CH}_{3}\right)_{2}\right), 2.66\left(\mathrm{~d}, J_{\mathrm{H}-\mathrm{P}}=\right.$ $\left.10.2 \mathrm{~Hz}, 4.2 \mathrm{H}, \mathrm{N}\left(\mathrm{CH}_{3}\right)_{2}\right), 2.69\left(\mathrm{~d}, J_{\mathrm{H}-\mathrm{P}}=10.3 \mathrm{~Hz}, 1.8 \mathrm{H}\right.$, $\left.\mathrm{N}\left(\mathrm{CH}_{3}\right)_{2}\right), 3.44-3.52(\mathrm{~m}, 0.9 \mathrm{H}, \mathrm{H}-2 \beta, \mathrm{H}-3 \beta, \mathrm{H}-5 \beta), 3.61$ (ddd, $\left.J=3.4,10.1,1.4\left(J_{\mathrm{H}-\mathrm{P}}\right) \mathrm{Hz}, 0.7 \mathrm{H}, \mathrm{H}-2 \alpha\right), 3.64-3.69$

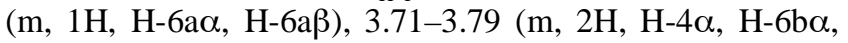

H-4 $\beta, \mathrm{H}-6 \mathrm{~b} \beta$ ), 3.88 (dd, $J=9.0,10.1 \mathrm{~Hz}, 0.7 \mathrm{H}, \mathrm{H}-3 \alpha$ ), 3.94 (ddd, $J=1.8,3.1,10.0 \mathrm{~Hz}, 0.7 \mathrm{H}, \mathrm{H}-5 \alpha), 4.49$ (d, $J=$ $12.0 \mathrm{~Hz}, 0.7 \mathrm{H}, \mathrm{OCHPh}), 4.51(\mathrm{~d}, J=12.1 \mathrm{~Hz}, 0.3 \mathrm{H}$, $\mathrm{OC} H \mathrm{Ph}), 4.561(\mathrm{~d}, J=12.1 \mathrm{~Hz}, 0.3 \mathrm{H}, \mathrm{OC} H \mathrm{Ph}), 4.562$ (d, $J=10.7 \mathrm{~Hz}, 0.7 \mathrm{H}, \mathrm{OCHPh}), 4.60$ (d, $J=10.9 \mathrm{~Hz}, 0.3 \mathrm{H}$, OCHPh), $4.61(\mathrm{~d}, J=12.0 \mathrm{~Hz}, 0.7 \mathrm{H}, \mathrm{OC} H \mathrm{Ph}), 4.80(\mathrm{~d}, J=$ $10.9 \mathrm{~Hz}, 0.3 \mathrm{H}$, OCHPh), $4.81(\mathrm{~d}, J=10.7 \mathrm{~Hz}, 0.7 \mathrm{H}$, $\mathrm{OCHPh}), 4.83(\mathrm{~d}, J=10.3 \mathrm{~Hz}, 0.3 \mathrm{H}, \mathrm{OCHPh}), 4.86(\mathrm{~d}, J=$ $10.8 \mathrm{~Hz}, 0.7 \mathrm{H}$, OCHPh), 4.87 (d, $J=10.3 \mathrm{~Hz}, 0.3 \mathrm{H}$, OCHPh), 4.90 (d, $J=10.8 \mathrm{~Hz}, 0.7 \mathrm{H}, \mathrm{OCHPh}), 5.00$ (dd, $J$ $\left.=7.5,7.6\left(J_{\mathrm{H}-\mathrm{P}}\right) \mathrm{Hz}, 0.3 \mathrm{H}, \mathrm{H}-1 \beta\right), 5.75(\mathrm{dd}, J=3.4,7.9$ $\left.\left(J_{\mathrm{H}-\mathrm{P}}\right) \mathrm{Hz}, \quad 0.7 \mathrm{H}, \mathrm{H}-1 \alpha\right), \quad 7.10-7.17 \quad(\mathrm{~m}, \quad 3 \mathrm{H}, \quad \mathrm{Ar}-\mathrm{H})$, 7.26-7.38 (m, 12H, Ar-H); $\left.{ }^{13} \mathrm{C} \mathrm{NMR} \mathrm{(126} \mathrm{MHz,} \mathrm{CDCl}_{3}\right) \delta$ $36.3,36.36,36.41,36.45,36.48,64.2\left(\mathrm{~d}, J_{\mathrm{C}-\mathrm{P}}=7.3 \mathrm{~Hz}\right)$, $67.2\left(\mathrm{~d}, J_{\mathrm{C}-\mathrm{P}}=7.9 \mathrm{~Hz}\right), 68.2,68.3,72.7,73.5,73.6,75.0$, $75.2,75.3,75.5,75.6,77.4,77.9,80.4,83.0,93.5\left(\mathrm{~d}, J_{\mathrm{C}-\mathrm{P}}=\right.$ $4.0 \mathrm{~Hz}, \mathrm{C}-1 \alpha), 96.1\left(\mathrm{~d}, J_{\mathrm{C}-\mathrm{P}}=4.5 \mathrm{~Hz}, \mathrm{C}-1 \beta\right), 127.66$, 127.72, 127.8, 127.88, 127.91, 128.06, 128.11, 128.3, 128.4, $128.45,128.47,137.7,137.76,137.81,137.87,137.94 ;{ }^{31} \mathrm{P}$ NMR $\left(109 \mathrm{MHz}, \mathrm{CDCl}_{3}\right) \delta 19.41(\alpha), 20.01(\beta)$; FABHRMS $m / z$ calcd for $\mathrm{C}_{31} \mathrm{H}_{41} \mathrm{~N}_{5} \mathrm{O}_{6} \mathrm{P}(\mathrm{M}+\mathrm{H})^{+} 610.2795$, found 610.2795 .

\subsection{Glycosidations of 2-azido-3,4,6-tri- $O$-benzyl-2- deoxyglucosyl diphenyl phosphate $2 \mathrm{a}$}

4.2.1. Typical procedure for glycosidation of 2 -azido-2deoxyglucopyranosyl donors: methyl 4-O-(2-azido-3,4,6tri- $O$-benzyl-2-deoxy-D-glucopyranosyl)-2,3,6-tri- $O$-benzyl- $\alpha$-D-glucopyranoside (10).

TMSOTf in $\mathrm{CH}_{2} \mathrm{Cl}_{2}(1.0 \mathrm{M}, 0.15 \mathrm{~mL}, 0.15 \mathrm{mmol})$ was added to a stirred solution of diphenyl phosphate $\mathbf{2 a}(\alpha: \beta=$ 72:28) (70.8 mg, $0.10 \mathrm{mmol})$ and alcohol 8 (51.1 mg, 0.11 $\mathrm{mmol})$ in EtCN $(1.5 \mathrm{~mL})$ at $-78{ }^{\circ} \mathrm{C}$. After stirring at this temperature for $2 \mathrm{~h}$, the reaction was quenched with $\mathrm{Et}_{3} \mathrm{~N}$ $(0.1 \mathrm{~mL})$. The reaction mixture was poured into a two-layer mixture of AcOEt $(2 \mathrm{~mL})$ and $\mathrm{NaHCO}_{3}(3 \mathrm{~mL})$, and the whole was extracted with AcOEt $(20 \mathrm{~mL})$. The organic layer was successively washed with saturated aqueous $\mathrm{NaHCO}_{3}(5 \mathrm{~mL})$ and brine $(2 \times 5 \mathrm{~mL})$, and dried over anhydrous $\mathrm{Na}_{2} \mathrm{SO}_{4}$. Filtration and evaporation in vacuo furnished the crude product $(108.9 \mathrm{mg})$, from which an anomeric mixture of disaccharide $10(82.6 \mathrm{mg}, 90 \%, \alpha: \beta=$ 6:94) was obtained as a colorless oil after column chromatography (silica gel $6 \mathrm{~g}, 5: 1$ hexane/AcOEt). The anomeric ratio of the disaccharide was determined by HPLC analysis [column, Zorbax ${ }^{\circledR}$ Sil, $4.6 \times 250 \mathrm{~mm}$; eluent, 7:1 hexane/AcOEt; flow rate, $1.0 \mathrm{~mL} / \mathrm{min}$; detection, 254 $\mathrm{nm} ; t_{\mathrm{R}}(\alpha$-anomer $)=56.6 \mathrm{~min}, t_{\mathrm{R}}(\beta$-anomer $\left.)=63.5 \mathrm{~min}\right]$. The $\alpha$ - and $\beta$-glycosides were separated by flash column chromatography with 6:1 hexane/AcOEt.

The following work-up may be employed in those cases where the highly reactive primary alcohol (7 or 12 ) was used as an acceptor. It serves only to remove the imidate as its hydrolyzed product. After the reaction was quenched with $\mathrm{Et}_{3} \mathrm{~N}(0.1 \mathrm{~mL})$, the mixture was diluted with AcOEt $(20 \mathrm{~mL})$. The whole was successively washed with $10 \%$ aqueous $\mathrm{HCl}(5 \mathrm{~mL}), \mathrm{H}_{2} \mathrm{O}(5 \mathrm{~mL})$, saturated aqueous $\mathrm{NaHCO}_{3}(5 \mathrm{~mL})$ and brine $(2 \times 5 \mathrm{~mL})$, and dried over 
anhydrous $\mathrm{Na}_{2} \mathrm{SO}_{4}$. After filtration, the filtrate was concentrated in vacuo to yield the crude product containing propionated acceptor alcohol.

Data for $\beta$-anomer (10 $\beta)$ : TLC $R_{f}=0.49$ (2:1 hexane/ AcOEt); $[\alpha]_{\mathrm{D}}{ }^{15}-11.4^{\circ}$ (c 1.09, $\left.\mathrm{CHCl}_{3}\right)$; IR $\left(\mathrm{CHCl}_{3}\right) 3009$, 2910, 2870, 2112, 1496, 1454, 1361, 1277, 1087, $750 \mathrm{~cm}^{-1}$; ${ }^{1} \mathrm{H}$ NMR $\left(500 \mathrm{MHz}, \mathrm{CDCl}_{3}\right) \delta 3.18$ (ddd, $J=1.7,4.3,9.6$ $\left.\mathrm{Hz}, 1 \mathrm{H}, \mathrm{H}-5^{\prime}\right), 3.24$ (dd, $\left.J=8.9,9.8 \mathrm{~Hz}, 1 \mathrm{H}, \mathrm{H}-3^{\prime}\right), 3.31$ (dd, $\left.J=8.0,9.8 \mathrm{~Hz}, 1 \mathrm{H}, \mathrm{H}-2^{\prime}\right), 3.38\left(\mathrm{~s}, 3 \mathrm{H}, \mathrm{OCH}_{3}\right), 3.47-3.50$ (m, 2H, H-2, H-6'a), 3.59 (dd, $\left.J=8.9,9.6 \mathrm{~Hz}, 1 \mathrm{H}, \mathrm{H}-4^{\prime}\right)$, 3.62 (dd, $\left.J=1.7,11.1 \mathrm{~Hz}, 1 \mathrm{H}, \mathrm{H}-6^{\prime} \mathrm{b}\right), 3.70$ (dd, $J=1.6$, $10.9 \mathrm{~Hz}, 1 \mathrm{H}, \mathrm{H}-6 \mathrm{a}$ ), 3.78 (ddd, $J=1.6,3.2,9.8 \mathrm{~Hz}, 1 \mathrm{H}, \mathrm{H}-$ 5), $3.89(\mathrm{dd}, J=8.9,9.5 \mathrm{~Hz}, 1 \mathrm{H}, \mathrm{H}-3), 3.92(\mathrm{dd}, J=3.2$, $10.9 \mathrm{~Hz}, 1 \mathrm{H}, \mathrm{H}-6 \mathrm{~b}), 3.96$ (dd, $J=8.9,9.8 \mathrm{~Hz}, 1 \mathrm{H}, \mathrm{H}-4)$, $4.26\left(\mathrm{~d}, J=8.0 \mathrm{~Hz}, 1 \mathrm{H}, \mathrm{H}-1^{\prime}\right), 4.35(\mathrm{~d}, J=12.1 \mathrm{~Hz}, 1 \mathrm{H}$, OCHPh), $4.40(\mathrm{~d}, J=12.1 \mathrm{~Hz}, 1 \mathrm{H}, \mathrm{OC} H \mathrm{Ph}), 4.47(\mathrm{~d}, J=$ $12.1 \mathrm{~Hz}, 1 \mathrm{H}, \mathrm{OC} H \mathrm{Ph}), 4.54$ (d, $J=11.0 \mathrm{~Hz}, 1 \mathrm{H}, \mathrm{OCHPh})$, 4.58 (d, $J=12.1 \mathrm{~Hz}, 1 \mathrm{H}, \mathrm{OCHPh}), 4.59$ (d, $J=3.6 \mathrm{~Hz}, 1 \mathrm{H}$, $\mathrm{H}-1), 4.67(\mathrm{~d}, J=12.1 \mathrm{~Hz}, 1 \mathrm{H}, \mathrm{OCHPh}), 4.74(\mathrm{~d}, J=12.1$ $\mathrm{Hz}, 1 \mathrm{H}, \mathrm{OCHPh}), 4.76$ (d, $J=11.0 \mathrm{~Hz}, 1 \mathrm{H}, \mathrm{OCHPh}), 4.781$ $(\mathrm{d}, J=11.4 \mathrm{~Hz}, 1 \mathrm{H}, \mathrm{OCHPh}), 4.782(\mathrm{~d}, J=10.7 \mathrm{~Hz}, 1 \mathrm{H}$, OCHPh), $4.82(\mathrm{~d}, J=10.7 \mathrm{~Hz}, 1 \mathrm{H}, \mathrm{OCHPh}), 5.02(\mathrm{~d}, J=$ $11.4 \mathrm{~Hz}, 1 \mathrm{H}, \mathrm{OCHPh}), 7.17-7.36(\mathrm{~m}, 30 \mathrm{H}, \mathrm{Ar}-\mathrm{H}) ;{ }^{13} \mathrm{C}$ NMR $\left(126 \mathrm{MHz}, \mathrm{CDCl}_{3}\right) \delta 55.3,66.9,68.2,68.6,69.7$, 73.3, 73.47, 73.51, 74.7, 75.19, 75.24, 75.4, 77.9, 79.1, 80.3, 83.3, 98.3 (C-1), 100.9 (C-1'), 127.0, 127.4, 127.5, 127.65, 127.67, 127.70, 127.72, 127.8, 127.95, 127.98, 128.1, 128.2, $128.3,128.36,128.42,128.5,137.8,137.9,138.0,138.3$, 138.4, 139.5; FAB-HRMS $\mathrm{m} / z$ calcd for $\mathrm{C}_{55} \mathrm{H}_{59} \mathrm{~N}_{3} \mathrm{O}_{10} \mathrm{Na}$ $(\mathrm{M}+\mathrm{Na})^{+}$944.4098, found 944.4083; Anal. calcd for: $\mathrm{C}_{55} \mathrm{H}_{59} \mathrm{~N}_{3} \mathrm{O}_{10}$ : C, 71.64; H, 6.45; N, 4.56, found $\mathrm{C}, 71.45 ; \mathrm{H}$, 6.45; N, 4.55. Data for $\alpha$-anomer $(\mathbf{1 0} \alpha)$ : TLC $R_{f}=0.54(2: 1$ hexane/AcOEt); $[\alpha]_{\mathrm{D}}{ }^{15}+39.1^{\circ}\left(c 0.78, \mathrm{CHCl}_{3}\right) ; \mathrm{IR}\left(\mathrm{CHCl}_{3}\right)$ 3013, 2910, 2870, 2112, 1602, 1454, 1361, 1221, 1049, 713 $\mathrm{cm}^{-1}$; ${ }^{1} \mathrm{H}$ NMR $\left(500 \mathrm{MHz}, \mathrm{CDCl}_{3}\right) \delta 3.27$ (dd, $J=3.9,10.4$ $\mathrm{Hz}, 1 \mathrm{H}, \mathrm{H}-2^{\prime}$ ), 3.34 (brd, $\left.J=11.0 \mathrm{~Hz}, 1 \mathrm{H}, \mathrm{H}-6^{\prime} \mathrm{a}\right), 3.38$ (s, $\left.3 \mathrm{H}, \mathrm{OCH}_{3}\right), 3.52\left(\mathrm{dd}, J=1.4,11.0 \mathrm{~Hz}, 1 \mathrm{H}, \mathrm{H}-6^{\prime} \mathrm{b}\right), 3.57$ (dd, $J=3.6,9.6 \mathrm{~Hz}, 1 \mathrm{H}, \mathrm{H}-2), 3.65(\mathrm{dd}, J=1.9,11.0 \mathrm{~Hz}, 1 \mathrm{H}$, H-6a), 3.66-3.70 (m, 2H, H-4', H-5'), 3.72 (dd, $J=4.3$, $11.0 \mathrm{~Hz}, 1 \mathrm{H}, \mathrm{H}-6 \mathrm{~b}$ ), 3.79 (ddd, $J=1.9,4.3,10.0 \mathrm{~Hz}, 1 \mathrm{H}$, H-5), 3.86 (m, 1H, H-3'), 3.91 (dd, $J=8.6,10.0 \mathrm{~Hz}, 1 \mathrm{H}, \mathrm{H}-$ 4), 4.08 (dd, $J=8.6,9.6 \mathrm{~Hz}, 1 \mathrm{H}, \mathrm{H}-3), 4.25(\mathrm{~d}, J=12.1 \mathrm{~Hz}$, $1 \mathrm{H}, \mathrm{OCHPh}), 4.45(\mathrm{~d}, J=10.9 \mathrm{~Hz}, 1 \mathrm{H}, \mathrm{OCHPh}), 4.49$ (d, $J$ $=12.1 \mathrm{~Hz}, 1 \mathrm{H}, \mathrm{OCHPh}), 4.50\left(\mathrm{~s}, 2 \mathrm{H}, \mathrm{OCH}_{2} \mathrm{Ph}\right), 4.61(\mathrm{~d}, J$ $=3.6 \mathrm{~Hz}, 1 \mathrm{H}, \mathrm{H}-1), 4.62(\mathrm{~d}, J=12.2 \mathrm{~Hz}, 1 \mathrm{H}, \mathrm{OCHPh})$, $4.74(\mathrm{~d}, J=10.9 \mathrm{~Hz}, 1 \mathrm{H}, \mathrm{OCHPh}), 4.75(\mathrm{~d}, J=12.2 \mathrm{~Hz}, 1 \mathrm{H}$, OCHPh), 4.83 (d, $J=10.9 \mathrm{~Hz}, 1 \mathrm{H}, \mathrm{OC} H \mathrm{Ph}), 4.85(\mathrm{~d}, J=$ $10.9 \mathrm{~Hz}, 1 \mathrm{H}, \mathrm{OCHPh}), 4.86$ (d, $J=10.7 \mathrm{~Hz}, 1 \mathrm{H}, \mathrm{OCHPh})$, $5.10(\mathrm{~d}, J=10.7 \mathrm{~Hz}, 1 \mathrm{H}, \mathrm{OCHPh}), 5.73(\mathrm{~d}, J=3.9 \mathrm{~Hz}, 1 \mathrm{H}$, $\left.\mathrm{H}-1^{\prime}\right), 7.12$ (m, 2H, Ar-H), 7.20-7.35 (m, 28H, Ar-H); ${ }^{13} \mathrm{C}$ NMR $\left(126 \mathrm{MHz}, \mathrm{CDCl}_{3}\right) \delta$ 55.3, 63.3, 67.9, 69.3, 69.5, 71.4, 73.27, 73.31, 73.5, 74.9, 75.0, 75.3, 78.1, 80.1, 80.5, 82.0, 97.69 (C-1'), $97.73(\mathrm{C}-1), 127.2,127.4,127.45$, 127.53, 127.66, 127.71, 127.8, 127.9, 128.1, 128.26, 128.32, $128.4,128.5,137.8,137.9,138.0,138.1,138.2,138.7$; FAB-HRMS $m / z$ calcd for $\mathrm{C}_{55} \mathrm{H}_{59} \mathrm{~N}_{3} \mathrm{O}_{10} \mathrm{Na} \quad(\mathrm{M}+\mathrm{Na})^{+}$ 944.4098, found 944.4083 .

4.2.2. Methyl 6-O-(2-azido-3,4,6-tri- $O$-benzyl-2-deoxy-Dglucopyranosyl)-2,3,4-tri- $O$-benzyl- $\alpha$-D-glucopyranoside
(9). The glycosidation was performed according to the typical procedure $\left(1.5 \mathrm{~mL} \mathrm{EtCN},-78{ }^{\circ} \mathrm{C}, 1.5 \mathrm{~h}\right)$ employing diphenyl phosphate $\mathbf{2 a}(70.8 \mathrm{mg}, 0.10 \mathrm{mmol})$, alcohol 7 (51.1 $\mathrm{mg}, 0.11 \mathrm{mmol})$, and TMSOTf $\left(1.0 \mathrm{M}\right.$ in $\mathrm{CH}_{2} \mathrm{Cl}_{2}$, $0.15 \mathrm{~mL}, 0.15 \mathrm{mmol})$. An anomeric mixture of disaccharide 9 (77.0 mg, 84\%, $\alpha: \beta=1: 99$ ) was obtained as a white solid from the crude product (108.7 $\mathrm{mg}$ ) after flash column chromatography (silica gel $6 \mathrm{~g}, 6: 1$ hexane/AcOEt with $1 \%$ $\left.\mathrm{Et}_{3} \mathrm{~N}\right)$, along with $\alpha$-imidate $\mathbf{1 1}(5.3 \mathrm{mg}, 5 \%)$ as a colorless oil. The anomeric ratio of the disaccharide was determined by HPLC analysis [column, Zorbax ${ }^{\circledR} \mathrm{Sil}, 4.6 \times 250 \mathrm{~mm}$; eluent, 5:1 hexane/AcOEt; flow rate, $1.0 \mathrm{~mL} / \mathrm{min}$; detection, $254 \mathrm{~nm} ; t_{\mathrm{R}}(\beta$-anomer $)=24.1 \mathrm{~min}, t_{\mathrm{R}}(\alpha$-anomer $)=34.7$ $\min ]$. The $\alpha$ - and $\beta$-glycosides were separated by flash column chromatography with $3: 1$ hexane/ $\mathrm{Et}_{2} \mathrm{O}$. Data for $\beta$ anomer $(9 \beta)$ : TLC $R_{f}=0.46(2: 1$ hexane/AcOEt), 0.30 (1:1 hexane/ $\mathrm{Et}_{2} \mathrm{O}$ ); mp $119.0-120.0{ }^{\circ} \mathrm{C}$ (colorless fine needles from AcOEt-hexane); $[\alpha]_{\mathrm{D}}{ }^{19}-5.27^{\circ}$ (c 1.15, $\mathrm{CHCl}_{3}$ ); IR $\left(\mathrm{CHCl}_{3}\right)$ 3009, 2930, 2868, 2112, 1496, 1454, 1359, 1265, 1222, 1068, $763 \mathrm{~cm}^{-1} ;{ }^{1} \mathrm{H}$ NMR $\left(500 \mathrm{MHz}, \mathrm{CDCl}_{3}\right) \delta 3.37$ $\left(\mathrm{m}, 1 \mathrm{H}, \mathrm{H}-5^{\prime}\right), 3.38\left(\mathrm{~s}, 3 \mathrm{H}, \mathrm{OCH}_{3}\right), 3.40(\mathrm{dd}, J=8.7,9.8 \mathrm{~Hz}$, $\left.1 \mathrm{H}, \mathrm{H}-3^{\prime}\right), 3.45$ (dd, $\left.J=7.7,9.8 \mathrm{~Hz}, 1 \mathrm{H}, \mathrm{H}-2^{\prime}\right), 3.55$ (dd, $J$ $=3.5,9.6 \mathrm{~Hz}, 1 \mathrm{H}, \mathrm{H}-2), 3.575(\mathrm{dd}, J=8.7,9.4 \mathrm{~Hz}, 1 \mathrm{H}, \mathrm{H}-$ $\left.4^{\prime}\right), 3.576(\mathrm{dd}, J=8.9,10.0 \mathrm{~Hz}, 1 \mathrm{H}, \mathrm{H}-4), 3.64-3.71(\mathrm{~m}$, $3 \mathrm{H}, \mathrm{H}-6 \mathrm{a}, \mathrm{H}-6$ ' a, H-6'b), 3.81 (ddd, $J=1.6,4.3,10.0 \mathrm{~Hz}$, $1 \mathrm{H}, \mathrm{H}-5), 4.00(\mathrm{dd}, J=8.9,9.6 \mathrm{~Hz}, 1 \mathrm{H}, \mathrm{H}-3), 4.12(\mathrm{dd}, J=$ $1.6,10.9 \mathrm{~Hz}, 1 \mathrm{H}, \mathrm{H}-6 \mathrm{~b}), 4.16$ (d, $\left.J=7.7 \mathrm{~Hz}, 1 \mathrm{H}, \mathrm{H}-1^{\prime}\right)$, $4.51(\mathrm{~d}, J=12.1 \mathrm{~Hz}, 1 \mathrm{H}, \mathrm{OCHPh}), 4.55(\mathrm{~d}, J=12.4 \mathrm{~Hz}, 1 \mathrm{H}$, $\mathrm{OCHPh}), 4.57$ (d, $J=12.1 \mathrm{~Hz}, 1 \mathrm{H}, \mathrm{OCHPh}), 4.62$ (d, $J=$ $3.5 \mathrm{~Hz}, 1 \mathrm{H}, \mathrm{H}-1), 4.65$ (d, $J=12.1 \mathrm{~Hz}, 1 \mathrm{H}, \mathrm{OCHPh}), 4.66$ $(\mathrm{d}, J=11.1 \mathrm{~Hz}, 1 \mathrm{H}, \mathrm{OCHPh}), 4.77-4.80(\mathrm{~m}, 3 \mathrm{H}$, $\mathrm{OCHPh} \times 3), 4.84(\mathrm{~d}, J=11.0 \mathrm{~Hz}, 1 \mathrm{H}, \mathrm{OCHPh}), 4.86(\mathrm{~d}, J=$ $10.7 \mathrm{~Hz}, 1 \mathrm{H}, \mathrm{OCHPh}), 4.93$ (d, $J=11.1 \mathrm{~Hz}, 1 \mathrm{H}, \mathrm{OCHPh})$, $4.98(\mathrm{~d}, J=11.0 \mathrm{~Hz}, 1 \mathrm{H}, \mathrm{OCHPh}), 7.17(\mathrm{~m}, 2 \mathrm{H}, \mathrm{Ar}-\mathrm{H})$, 7.24-7.36 (m, 28H, Ar-H); ${ }^{13} \mathrm{C}$ NMR (126 MHz, $\left.\mathrm{CDCl}_{3}\right) \delta$ $55.2,66.4,68.4,68.7,69.7,73.4,74.8,75.0,75.2,75.6$, $75.7,77.75,77.79,79.8,82.1,83.3,98.2$ (C-1), 102.1 (C$\left.1^{\prime}\right), 127.5,127.59,127.63,127.7,127.8,127.85,127.87$, $128.0,128.06,128.14,128.3,128.35,128.42,128.44,137.9$, 138.1, 138.2, 138.4, 138.8; FAB-HRMS $\mathrm{m} / \mathrm{z}$ calcd for $\mathrm{C}_{55} \mathrm{H}_{59} \mathrm{~N}_{3} \mathrm{O}_{10} \mathrm{Na}(\mathrm{M}+\mathrm{Na})^{+}$944.4098, found 944.4080; Anal. calcd for: $\mathrm{C}_{55} \mathrm{H}_{59} \mathrm{~N}_{3} \mathrm{O}_{10}: \mathrm{C}, 71.64 ; \mathrm{H}, 6.45 ; \mathrm{N}, 4.56$, found $\mathrm{C}$, 71.67; H, 6.44; N 4.49. Data for $\alpha$-anomer $(9 \alpha)$ : TLC $R_{f}=$ $0.44\left(2: 1\right.$ hexane/AcOEt), $0.26(1: 1$ hexane/Et $2 \mathrm{O}) ;[\alpha]_{\mathrm{D}}{ }^{24}$ $+84.7^{\circ}$ (c 1.18, $\mathrm{CHCl}_{3}$ ); IR (film) 3030, 2922, 2106, 1496, 1454, 1359, 1207, 1049, $736 \mathrm{~cm}^{-1} ;{ }^{1} \mathrm{H}$ NMR $(500 \mathrm{MHz}$, $\left.\mathrm{CDCl}_{3}\right) \delta 3.33\left(\mathrm{dd}, J=3.5,10.1 \mathrm{~Hz}, 1 \mathrm{H}, \mathrm{H}-2^{\prime}\right), 3.37$ (s, 3H, $\left.\mathrm{OCH}_{3}\right), 3.51-3.55$ (m, 2H, H-2, H-6'a), 3.56 (dd, $1 \mathrm{H}, J=$ 9.2, $9.4 \mathrm{~Hz}, \mathrm{H}-4), 3.63$ (dd, $\left.J=3.3,10.7 \mathrm{~Hz}, \mathrm{H}-6^{\prime} \mathrm{b}\right)$, 3.67-3.71 (2H, m, H-6a, H-4'), 3.74-3.78 (2H, m, H-5, H$\left.5^{\prime}\right), 3.83(\mathrm{dd}, J=4.6,11.4 \mathrm{~Hz}, 1 \mathrm{H}, \mathrm{H}-6 \mathrm{~b}), 3.92(\mathrm{dd}, J=8.8$, $\left.10.1 \mathrm{~Hz}, 1 \mathrm{H}, \mathrm{H}-3^{\prime}\right), 4.00$ (t, $\left.J=9.2 \mathrm{~Hz}, 1 \mathrm{H}, \mathrm{H}-3\right), 4.43$ (d, $J$ $=12.1 \mathrm{~Hz}, 1 \mathrm{H}, \mathrm{OCHPh}), 4.49(\mathrm{~d}, J=10.9 \mathrm{~Hz}, 1 \mathrm{H}, \mathrm{OC} H \mathrm{Ph})$, 4.56-4.61 (m, 3H, H-1, OCHPh $\times 2), 4.66(\mathrm{~d}, J=12.0 \mathrm{~Hz}$, $1 \mathrm{H}, \mathrm{OC} H \mathrm{Ph}), 4.78(\mathrm{~d}, J=12.0 \mathrm{~Hz}, 1 \mathrm{H}, \mathrm{OC} H \mathrm{Ph}), 4.79(\mathrm{~d}, J$ $=10.9 \mathrm{~Hz}, 1 \mathrm{H}, \mathrm{OC} H \mathrm{Ph}), 4.80(\mathrm{~d}, J=10.9 \mathrm{~Hz}, 1 \mathrm{H}, \mathrm{OC} H \mathrm{Ph})$, $4.83(\mathrm{~d}, J=10.8 \mathrm{~Hz}, 1 \mathrm{H}, \mathrm{OCHPh}), 4.86(\mathrm{~d}, J=10.8 \mathrm{~Hz}, 1 \mathrm{H}$, OCHPh), $4.94(\mathrm{~d}, J=11.2 \mathrm{~Hz}, 1 \mathrm{H}, \mathrm{OCHPh}), 4.98(\mathrm{~d}, J=$ $10.9 \mathrm{~Hz}, 1 \mathrm{H}, \mathrm{OCHPh}), 5.01$ (d, $\left.J=3.5 \mathrm{~Hz}, 1 \mathrm{H}, \mathrm{H} 1^{\prime}\right), 7.13$ (m, 2H, Ar-H), 7.24-7.37 (m, 28H, Ar-H); ${ }^{13} \mathrm{C}$ NMR $(100$ $\left.\mathrm{MHz}, \mathrm{CDCl}_{3}\right) \delta$ 55.2, 63.5, 66.4, 68.1, 69.9, 70.7, 73.4, 
73.5, 74.9, 75.2, 75.8, 77.2, 77.7, 78.2, 79.8, 80.0, 82.0, 97.9 (C-1), $98.2\left(\mathrm{C}-1^{\prime}\right), 127.5,127.6,127.7,127.76,127.80$, 128.0, 128.1, 128.29, 128.31, 128.4, 137.7, 137.8, 137.95, 138.04, 138.2, 138.6; FAB-HRMS $\mathrm{m} / \mathrm{z}$ calcd for $\mathrm{C}_{55} \mathrm{H}_{59} \mathrm{~N}_{3} \mathrm{O}_{10} \mathrm{Na}(\mathrm{M}+\mathrm{Na})^{+}$944.4098, found 944.4109.

Data for methyl 6-O-[1-(2-azido-3,4,6-tri- $O$-benzyl-2deoxy- $\alpha$-D-glucopyranosyl)iminopropyl]-2,3,4-tri- $O$-benzyl- $\alpha$-D-glucopyranoside (11): TLC $R_{f}=0.49$ (2:1 hexane/ AcOEt); $[\alpha]_{\mathrm{D}}{ }^{20}+47.5^{\circ}$ (c $0.40, \mathrm{CHCl}_{3}$ ); IR (film) 3030, 2922, 2106, 1664, 1496, 1454, 1359, 1211, $1089 \mathrm{~cm}^{-1} ;{ }^{1} \mathrm{H}$ NMR $\left(500 \mathrm{MHz}, \mathrm{CDCl}_{3}\right) \delta 1.15(\mathrm{t}, J=7.6 \mathrm{~Hz}, 3 \mathrm{H}$, $\left.\mathrm{CH}_{2} \mathrm{CH}_{3}\right), 2.36\left(\mathrm{dq}, J=14.6,7.6 \mathrm{~Hz}, 1 \mathrm{H}, \mathrm{CHCH}_{3}\right), 2.38$ (dq, $\left.J=14.6,7.6 \mathrm{~Hz}, 1 \mathrm{H}, \mathrm{CHCH}_{3}\right), 3.37\left(\mathrm{~s}, 3 \mathrm{H}, \mathrm{OCH}_{3}\right), 3.52$ $\left(\mathrm{dd}, J=4.1,10.0 \mathrm{~Hz}, 1 \mathrm{H}, \mathrm{H}-2^{\prime}\right), 3.54(\mathrm{dd}, J=3.6,9.5 \mathrm{~Hz}$, $1 \mathrm{H}, \mathrm{H}-2), 3.56$ (dd, $J=1.7,10.7 \mathrm{~Hz}, 1 \mathrm{H}, \mathrm{H}-6$ ' a), 3.62 (dd, $J$ $=9.0,10.0 \mathrm{~Hz}, 1 \mathrm{H}, \mathrm{H}-4), 3.73(\mathrm{dd}, J=3.5,10.7 \mathrm{~Hz}, 1 \mathrm{H}, \mathrm{H}-$ $6^{\prime}$ b), 3.78 (dd, $\left.J=9.0,9.9 \mathrm{~Hz}, 1 \mathrm{H}, \mathrm{H}-4^{\prime}\right), 3.87$ (ddd, $J=1.8$, 4.1, $10.0 \mathrm{~Hz}, 1 \mathrm{H}, \mathrm{H}-5), 4.00$ (dd, $J=9.0,9.5 \mathrm{~Hz}, 1 \mathrm{H}, \mathrm{H}-3)$, 4.08 (ddd, $\left.J=1.7,3.5,9.9 \mathrm{~Hz}, 1 \mathrm{H}, \mathrm{H}-5^{\prime}\right), 4.09$ (dd, $J=9.0$, $\left.10.0 \mathrm{~Hz}, 1 \mathrm{H}, \mathrm{H}-3^{\prime}\right), 4.23$ (dd, $\left.J=4.1,12.3 \mathrm{~Hz}, 1 \mathrm{H}, \mathrm{H}-6 \mathrm{a}\right)$, $4.30(\mathrm{dd}, J=1.8,12.3 \mathrm{~Hz}, 1 \mathrm{H}, \mathrm{H}-6 \mathrm{~b}), 4.46(\mathrm{~d}, J=12.2 \mathrm{~Hz}$, $1 \mathrm{H}, \mathrm{OC} H \mathrm{Ph}), 4.51(\mathrm{~d}, J=10.7 \mathrm{~Hz}, 1 \mathrm{H}, \mathrm{OCHPh}), 4.59$ (d, $J$ $=10.6 \mathrm{~Hz}, 1 \mathrm{H}, \mathrm{OCH} \mathrm{Ph}), 4.60(\mathrm{~d}, J=3.6 \mathrm{~Hz}, 1 \mathrm{H}, \mathrm{H}-1)$, $4.62(\mathrm{~d}, J=12.2 \mathrm{~Hz}, 1 \mathrm{H}, \mathrm{OCHPh}), 4.66(\mathrm{~d}, J=12.1 \mathrm{~Hz}, 1 \mathrm{H}$, OCHPh), $4.73(\mathrm{~d}, J=10.7 \mathrm{~Hz}, 1 \mathrm{H}, \mathrm{OCHPh}), 4.789(\mathrm{~d}, J=$ $10.7 \mathrm{~Hz}, 1 \mathrm{H}, \mathrm{OCHPh}), 4.790$ (d, $J=12.1 \mathrm{~Hz}, 1 \mathrm{H}, \mathrm{OCHPh})$, $4.80(\mathrm{~d}, J=10.7 \mathrm{~Hz}, 1 \mathrm{H}, \mathrm{OCHPh}), 4.82(\mathrm{~d}, J=10.8 \mathrm{~Hz}, 1 \mathrm{H}$, OCHPh), 4.84 (d, $J=10.6 \mathrm{~Hz}, 1 \mathrm{H}, \mathrm{OCHPh}), 4.97$ (d, $J=$ $10.8 \mathrm{~Hz}, 1 \mathrm{H}, \mathrm{OC} H \mathrm{Ph}), 5.20\left(\mathrm{~d}, J=4.1 \mathrm{~Hz}, 1 \mathrm{H}, \mathrm{H}-1^{\prime}\right)$, 7.12-7.18 (m, 3H, Ar-H), 7.22-7.37 (m, 27H, Ar-H); ${ }^{13} \mathrm{C}$ NMR $\left(126 \mathrm{MHz}, \mathrm{CDCl}_{3}\right) \delta 10.7,22.9,55.1,64.2,64.7$, 68.7, 71.2, 73.4, 73.5, 75.09, 75.14, 75.2, 75.9, 77.8, 78.9, $80.0,80.9,82.1,83.2\left(\mathrm{C}-1^{\prime}\right), 98.1$ (C-1), 127.6, 127.68, $127.74,127.76,127.79,127.87,127.91,128.0,128.1$, $128.35,128.41,128.5,137.9,138.0,138.07,138.13,138.2$, 138.7, 168.8; FAB-HRMS $\mathrm{m} / \mathrm{z}$ calcd for $\mathrm{C}_{58} \mathrm{H}_{65} \mathrm{~N}_{4} \mathrm{O}_{10}$ $(\mathrm{M}+\mathrm{H})^{+}$977.4700, found 977.4721.

\subsubsection{6-O-(2-Azido-3,4,6-tri- $O$-benzyl-2-deoxy-D-gluco-} pyranosyl)-1,2:3,4-di- $O$-isopropylidene- $\alpha$-D-galactopyranose (17). ${ }^{12 a}$ The glycosidation was performed according to the typical procedure $\left(1.5 \mathrm{~mL} \mathrm{EtCN},-78{ }^{\circ} \mathrm{C}, 1.5 \mathrm{~h}\right)$ employing diphenyl phosphate $\mathbf{2 a}(70.8 \mathrm{mg}, 0.10 \mathrm{mmol})$, alcohol 12 (28.6 mg, $0.11 \mathrm{mmol})$, and TMSOTf (1.0 M in $\left.\mathrm{CH}_{2} \mathrm{Cl}_{2}, 0.15 \mathrm{~mL}, 0.15 \mathrm{mmol}\right)$. An anomeric mixture of disaccharide 17 (56.8 mg, 79\%, $\alpha: \beta=2: 98$ ) was obtained as a colorless oil from the crude product $(91.4 \mathrm{mg})$ after column chromatography (silica gel $8 \mathrm{~g}, 7: 1$ hexane/AcOEt with $\left.1 \% \mathrm{Et}_{3} \mathrm{~N}\right)$, along with $\alpha$-imidate $19(7.2 \mathrm{mg}, 9 \%)$ as a colorless syrup. The anomeric ratio of the product was determined by HPLC analysis [eluent, 6:1 hexane/THF; flow rate, $1.0 \mathrm{~mL} / \mathrm{min} ; t_{\mathrm{R}}(\alpha$-anomer $)=8.9 \mathrm{~min}, t_{\mathrm{R}}(\beta$ anomer $)=9.6 \mathrm{~min}]$. The $\alpha$ - and $\beta$-glycosides were separated by flash column chromatography with 30:1 toluene/acetone. Data for $\beta$-anomer $(\mathbf{1 7} \beta)$ : TLC $R_{f}=0.39$ (3:1 hexane/AcOEt), 0.49 (10:1 toluene/acetone); $[\alpha]_{\mathrm{D}}{ }^{16}$ $-47.9^{\circ}$ ( c 2.45, $\left.\mathrm{CHCl}_{3}\right)(\alpha: \beta=2: 98)$; IR (film) 2986, 2906, 2110, 1454, 1381, 1211, $1070 \mathrm{~cm}^{-1}$; ${ }^{1} \mathrm{H}$ NMR $(500 \mathrm{MHz}$, $\left.\mathrm{CDCl}_{3}\right) \delta 1.32\left(\mathrm{~s}, 3 \mathrm{H}, \mathrm{CCH}_{3}\right), 1.33\left(\mathrm{~s}, 3 \mathrm{H}, \mathrm{CCH}_{3}\right), 1.44(\mathrm{~s}$, $\left.3 \mathrm{H}, \mathrm{CCH}_{3}\right), 1.54$ (s, 3H, $\left.\mathrm{CCH}_{3}\right), 3.40-3.44$ (m, 3H, H-2', H-
4', H-5'), 3.63 (m, 1H, H-3'), 3.70 (dd, $J=4.1,11.1 \mathrm{~Hz}, 1 \mathrm{H}$, H-6'a), 3.73 (dd, $\left.J=2.2,11.1 \mathrm{~Hz}, 1 \mathrm{H}, \mathrm{H}-6^{\prime} \mathrm{b}\right), 3.79$ (m, 1H, H-6a), 4.04-4.09 (m, 2H, H-5, H-6b), 4.28 (dd, $J=1.2,7.8$ $\mathrm{Hz}, 1 \mathrm{H}, \mathrm{H}-4), 4.31$ (dd, $J=2.4,5.0 \mathrm{~Hz}, 1 \mathrm{H}, \mathrm{H}-2), 4.41$ (m, $\left.1 \mathrm{H}, \mathrm{H}-1^{\prime}\right), 4.53(\mathrm{~d}, J=12.1 \mathrm{~Hz}, 1 \mathrm{H}, \mathrm{OCHPh}), 4.54(\mathrm{~d}, J=$ $10.9 \mathrm{~Hz}, 1 \mathrm{H}, \mathrm{OCHPh}), 4.60$ (dd, $J=2.4,7.8 \mathrm{~Hz}, 1 \mathrm{H}, \mathrm{H}-3)$, $4.61(\mathrm{~d}, J=12.1 \mathrm{~Hz}, 1 \mathrm{H}, \mathrm{OCHPh}), 4.78(\mathrm{~d}, J=10.8 \mathrm{~Hz}, 1 \mathrm{H}$, OCHPh), 4.79 (d, $J=10.9 \mathrm{~Hz}, 1 \mathrm{H}, \mathrm{OCHPh}), 4.89$ (d, $J=$ $10.8 \mathrm{~Hz}, 1 \mathrm{H}, \mathrm{OCHPh}), 5.54$ (d, $J=5.0 \mathrm{~Hz}, 1 \mathrm{H}, \mathrm{H}-1), 7.16$ (m, 2H, Ar-H), 7.25-7.36 (m, 13H, Ar-H); ${ }^{13} \mathrm{C}$ NMR $(126$ $\left.\mathrm{MHz} \mathrm{CDCl}_{3}\right) \delta$ 24.4, 25.0, 25.97, 26.02, 66.4, 67.6, 68.5, $68.8,70.5,70.7,71.2,73.5,74.96,75.02,75.5,77.7,83.1$, $96.3(\mathrm{C}-1), 102.4\left(\mathrm{C}-1^{\prime}\right), 108.7,109.3,127.6,127.76$, $127.79,127.82,128.0,128.35,128.38,128.41,138.0$, 138.06, 138.08; FAB-HRMS $\mathrm{m} / z$ calcd for $\mathrm{C}_{39} \mathrm{H}_{47} \mathrm{~N}_{3} \mathrm{O}_{10} \mathrm{Na}$ $(\mathrm{M}+\mathrm{Na})^{+}$740.3159, found 740.3195; Anal. calcd for: $\mathrm{C}_{39} \mathrm{H}_{47} \mathrm{~N}_{3} \mathrm{O}_{10}$ : C, 65.26; H, 6.60; N, 5.85, found $\mathrm{C}, 65.26 ; \mathrm{H}$, 6.60; N, 5.83. Data for $\alpha$-anomer $(17 \alpha)$ : TLC $R_{f}=0.41(3: 1$ hexane/AcOEt), $0.53\left(10: 1\right.$ toluene/acetone); $[\alpha]_{\mathrm{D}}{ }^{17}+42.2^{\circ}$ (c $\left.0.49, \mathrm{CHCl}_{3}\right)$; IR $\left(\mathrm{CHCl}_{3}\right) 2924,2106,1454,1381,1255$, $1070 \mathrm{~cm}^{-1} ;{ }^{1} \mathrm{H}$ NMR $\left(500 \mathrm{MHz}, \mathrm{CDCl}_{3}\right) \delta 1.33(\mathrm{~s}, 3 \mathrm{H}$, $\left.\mathrm{CCH}_{3}\right), 1.34\left(\mathrm{~s}, 3 \mathrm{H}, \mathrm{CCH}_{3}\right), 1.43\left(\mathrm{~s}, 3 \mathrm{H}, \mathrm{CCH}_{3}\right), 1.53(\mathrm{~s}, 3 \mathrm{H}$, $\left.\mathrm{CCH}_{3}\right), 3.33\left(\mathrm{dd}, J=3.5,10.3 \mathrm{~Hz}, 1 \mathrm{H}, \mathrm{H}-2^{\prime}\right), 3.66(\mathrm{dd}, J=$ $\left.1.8,10.8 \mathrm{~Hz}, 1 \mathrm{H}, \mathrm{H}-6^{\prime} \mathrm{a}\right), 3.72$ (dd, $J=6.7,10.3 \mathrm{~Hz}, 1 \mathrm{H}, \mathrm{H}-$ 6a), 3.73-3.80 (m, 2H, H-4', H-6'b), 3.81 (dd, $J=6.4,10.3$ $\mathrm{Hz}, 1 \mathrm{H}, \mathrm{H}-6 \mathrm{~b}), 3.88$ (m, 1H, H-5'), 3.98-4.01 (m, 2H, H-5, H-3'), 4.31 (dd, $J=2.3,5.0 \mathrm{~Hz}, 1 \mathrm{H}, \mathrm{H}-2), 4.32(\mathrm{dd}, J=1.8$, $8.0 \mathrm{~Hz}, 1 \mathrm{H}, \mathrm{H}-3), 4.48$ (d, $J=12.1 \mathrm{~Hz}, 1 \mathrm{H}, \mathrm{OCHPh}), 4.53$ $(\mathrm{d}, J=11.1 \mathrm{~Hz}, 1 \mathrm{H}, \mathrm{OCHPh}), 4.61(\mathrm{dd}, J=2.3,8.0 \mathrm{~Hz}, 1 \mathrm{H}$, $\mathrm{H}-3), 4.64(\mathrm{~d}, J=12.1 \mathrm{~Hz}, 1 \mathrm{H}, \mathrm{OC} H \mathrm{Ph}), 4.79(\mathrm{~d}, J=11.1$ $\mathrm{Hz}, 1 \mathrm{H}, \mathrm{OCHPh}), 4.85$ (d, $J=11.5 \mathrm{~Hz}, 1 \mathrm{H}, \mathrm{OCHPh}), 4.87$ $(\mathrm{d}, J=11.5 \mathrm{~Hz}, 1 \mathrm{H}, \mathrm{OCHPh}), 4.99(\mathrm{~d}, J=3.5 \mathrm{~Hz}, 1 \mathrm{H}, \mathrm{H}-$ $\left.1^{\prime}\right), 5.51(\mathrm{~d}, J=5.0 \mathrm{~Hz}, 1 \mathrm{H}, \mathrm{H}-1), 7.16(\mathrm{~m}, 2 \mathrm{H}, \mathrm{Ar}-\mathrm{H})$, 7.24-7.37 (m, 13H, Ar-H); ${ }^{13} \mathrm{C}$ NMR (126 MHz, $\left.\mathrm{CDCl}_{3}\right) \delta$ 24.4, 24.9, 26.0, 26.1, 63.4, 66.2, 66.9, 68.2, 70.6, 70.66, $70.69,70.8,73.5,74.9,75.3,78.3,79.9,96.3$ (C-1), 98.3 $\left(\mathrm{C}-1^{\prime}\right), 108.6,109.3,127.70,127.73,127.8,127.9,128.0$, $128.38,128.43,137.9,138.1$; FAB-HRMS $\mathrm{m} / \mathrm{z}$ calcd for $\mathrm{C}_{39} \mathrm{H}_{47} \mathrm{~N}_{3} \mathrm{O}_{10} \mathrm{Na}(\mathrm{M}+\mathrm{Na})^{+} 740.3159$, found 740.3134 .

Data for 6- $O$-[1-(2-azido-3,4,6-tri- $O$-benzyl-2-deoxy- $\alpha$-Dglucopyranosyl)iminopropyl]-1,2:3,4-di- $O$-isopropylidene$\alpha$-D-galactopyranose (19): TLC $R_{f}=0.43$ (3:1 hexane/ AcOEt); $[\alpha]_{\mathrm{D}}{ }^{24}+4.68^{\circ}$ (c $0.28, \mathrm{CHCl}_{3}$ ); IR (film) 2924, 2106, 1658,1462,1213, $1072 \mathrm{~cm}^{-1}$; ${ }^{1} \mathrm{H}$ NMR $(500 \mathrm{MHz}$, $\left.\mathrm{CDCl}_{3}\right) \delta 1.16\left(\mathrm{t}, J=7.6 \mathrm{~Hz}, 3 \mathrm{H}, \mathrm{CH}_{2} \mathrm{CH}_{3}\right), 1.32(\mathrm{~s}, 6 \mathrm{H}$, $\left.\mathrm{CCH}_{3} \times 2\right), 1.44\left(\mathrm{~s}, 3 \mathrm{H}, \mathrm{CCH}_{3}\right), 1.50\left(\mathrm{~s}, 3 \mathrm{H}, \mathrm{CCH}_{3}\right), 2.34$ (dq, $\left.J=15.0,7.6 \mathrm{~Hz}, 1 \mathrm{H}, \mathrm{CHCH}_{3}\right), 2.38(\mathrm{dq}, J=15.0,7.6 \mathrm{~Hz}$, $1 \mathrm{H}, \mathrm{CHCH}_{3}$ ), 3.57 (dd, $\left.J=4.2,10.0 \mathrm{~Hz}, 1 \mathrm{H}, \mathrm{H}-2^{\prime}\right), 3.58$ (dd, $\left.J=1.8,10.8 \mathrm{~Hz}, 1 \mathrm{H}, \mathrm{H}-66^{\prime} \mathrm{a}\right), 3.74$ (dd, $J=3.5,10.8 \mathrm{~Hz}$, $\left.1 \mathrm{H}, \mathrm{H}-6^{\prime} \mathrm{b}\right), 3.78\left(\mathrm{t}, J=9.5 \mathrm{~Hz}, 1 \mathrm{H}, \mathrm{H}-4^{\prime}\right), 4.05$ (ddd, $J=$ 1.6, 5.3, 7.1 Hz, $1 \mathrm{H}, \mathrm{H}-5), 4.10(\mathrm{dd}, J=9.5,10.0 \mathrm{~Hz}, 1 \mathrm{H}$, H-3'), 4.10-4.14 (m, 2H, H-6a, H-5'), 4.27 (dd, $J=1.6,7.9$ $\mathrm{Hz}, 1 \mathrm{H}, \mathrm{H}-4), 4.31$ (dd, $J=2.4,5.0 \mathrm{~Hz}, 1 \mathrm{H}, \mathrm{H}-2), 4.33$ (dd, $J=5.3,11.2 \mathrm{~Hz}, 1 \mathrm{H}, \mathrm{H}-6 \mathrm{~b}), 4.47(\mathrm{~d}, J=12.2 \mathrm{~Hz}, 1 \mathrm{H}$, $\mathrm{OC} H \mathrm{Ph}), 4.53(\mathrm{~d}, J=10.9 \mathrm{~Hz}, 1 \mathrm{H}, \mathrm{OC} H \mathrm{Ph}), 4.60(\mathrm{dd}, J=$ $2.4,7.9 \mathrm{~Hz}, 1 \mathrm{H}, \mathrm{H}-3), 4.62$ (d, $J=12.2 \mathrm{~Hz}, 1 \mathrm{H}, \mathrm{OCHPh})$, $4.82(\mathrm{~d}, J=10.9 \mathrm{~Hz}, 1 \mathrm{H}, \mathrm{OCHPh}), 4.85(\mathrm{~d}, J=11.7 \mathrm{~Hz}, 1 \mathrm{H}$, $\mathrm{OCHPh}), 4.89(\mathrm{~d}, J=11.7 \mathrm{~Hz}, 1 \mathrm{H}, \mathrm{OCHPh}), 5.22(\mathrm{~d}, J=$ $\left.4.2 \mathrm{~Hz}, 1 \mathrm{H}, \mathrm{H}-1^{\prime}\right), 5.55$ (d, $\left.J=5.0 \mathrm{~Hz}, 1 \mathrm{H}, \mathrm{H}-1\right), 7.17$ (m, 2H, Ar-H), 7.24-7.38 (m, 13H, Ar-H); ${ }^{13} \mathrm{C}$ NMR (126 MHz, 
$\left.\mathrm{CDCl}_{3}\right) \delta 10.7,23.0,24.4,25.0,26.0,26.1,29.7,64.0,65.0$ $66.1,68.7,70.7,71.2,71.3,73.5,75.0,75.3,79.0,80.8$, $83.4\left(\mathrm{C}-1^{\prime}\right), 96.3(\mathrm{C}-1), 108.6,109.5,127.65,127.70$, 127.76, 127.80, 127.9, 128.1, 128.3, 128.38, 128.43, 138.0, 138.1, 138.2, 168.0; FAB-HRMS $m / z$ calcd for $\mathrm{C}_{42} \mathrm{H}_{53} \mathrm{~N}_{4} \mathrm{O}_{10}$ $(\mathrm{M}+\mathrm{H})^{+} 773.3762$, found 773.3770 .

\subsubsection{Methyl 2-O-(2-azido-3,4,6-tri- $O$-benzyl-2-deoxy-D- glucopyranosyl)-3,4,6-tri- $O$-benzyl- $\beta$-D-glucopyranoside}

(18). ${ }^{12 \mathrm{~b}}$ The glycosidation was performed according to the typical procedure $\left(1.5 \mathrm{~mL} \mathrm{EtCN},-78{ }^{\circ} \mathrm{C}, 2 \mathrm{~h}\right)$ employing diphenyl phosphate $\mathbf{2 a}(70.8 \mathrm{mg}, 0.10 \mathrm{mmol})$, alcohol $\mathbf{1 3}$ (51.1 $\mathrm{mg}, 0.11 \mathrm{mmol})$, and TMSOTf $\left(1.0 \mathrm{M}\right.$ in $\mathrm{CH}_{2} \mathrm{Cl}_{2}$, $0.15 \mathrm{~mL}, 0.15 \mathrm{mmol}$ ). An anomeric mixture of disaccharide $18(83.6 \mathrm{mg}, 91 \%, \alpha: \beta=9: 91)$ was obtained as a colorless oil from the crude product (107.4 mg) after column chromatography (silica gel $5 \mathrm{~g}, 5: 1$ hexane/AcOEt). The anomeric ratio of the product was determined by HPLC analysis [eluent, 5:1 hexane/AcOEt; flow rate, $1.0 \mathrm{~mL} / \mathrm{min}$; $t_{\mathrm{R}}(\alpha$-anomer $)=12.6 \mathrm{~min}, t_{\mathrm{R}}(\beta$-anomer $\left.)=16.3 \mathrm{~min}\right]$. The $\alpha$ - and $\beta$-glycosides were separated by flash column chromatography with 6:1 hexane/AcOEt. Data for $\beta$ anomer $(\mathbf{1 8} \beta):^{12 \mathrm{~b}}$ TLC $R_{f}=0.61(2: 1$ hexane/AcOEt); $\mathrm{mp}$ 83.5-84.5 ${ }^{\circ} \mathrm{C}$ (colorless needles from AcOEt-hexane); $[\alpha]_{\mathrm{D}}{ }^{28}-17.9^{\circ}\left(c 1.00, \mathrm{CHCl}_{3}\right)$; IR $(\mathrm{KBr}) 3030,2908,2868$, 2112, 1496, 1452, 1359, 1269, 1062, $750 \mathrm{~cm}^{-1}$; ${ }^{1} \mathrm{H}$ NMR $\left(500 \mathrm{MHz}, \mathrm{CDCl}_{3}\right) \delta 3.34\left(\mathrm{~m}, 1 \mathrm{H}, \mathrm{H}_{-} 5^{\prime}\right), 3.39$ (dd, $J=9.1$, $\left.9.5 \mathrm{~Hz}, 1 \mathrm{H}, \mathrm{H}-3^{\prime}\right), 3.46$ (dd, $\left.J=8.1,9.5 \mathrm{~Hz}, 1 \mathrm{H}, \mathrm{H}-2^{\prime}\right), 3.48$ (s, 3H, OCH $\left.H_{3}\right), 3.49$ (m, 1H, H-5), 3.64-3.78 (m, 7H, H-3, H-4, H-6a, H-6b, H-4', H-6'a, H-6'b), 3.80 (dd, $J=7.2,8.9$ $\mathrm{Hz}, 1 \mathrm{H}, \mathrm{H}-2), 4.37$ (d, $J=7.2 \mathrm{~Hz}, 1 \mathrm{H}, \mathrm{H}-1), 4.53-4.59$ (m, $4 \mathrm{H}, \mathrm{OC} H \mathrm{Ph} \times 4), 4.61-4.66(\mathrm{~m}, 2 \mathrm{H}, \mathrm{OC} H \mathrm{Ph} \times 2), 4.71(\mathrm{~d}, J=$ $\left.8.1 \mathrm{~Hz}, 1 \mathrm{H}, \mathrm{H}-1^{\prime}\right), 4.78$ (d, $\left.J=10.5 \mathrm{~Hz}, 1 \mathrm{H}, \mathrm{OCHPh}\right), 4.79$ $(\mathrm{d}, J=10.9 \mathrm{~Hz}, 1 \mathrm{H}, \mathrm{OCHPh}), 4.81(\mathrm{~d}, J=10.8 \mathrm{~Hz}, 1 \mathrm{H}$, OCHPh), $4.85(\mathrm{~d}, J=10.8 \mathrm{~Hz}, 1 \mathrm{H}, \mathrm{OCHPh}), 4.89$ (d, $J=$ $10.6 \mathrm{~Hz}, 1 \mathrm{H}, \mathrm{OCHPh}), 4.95(\mathrm{~d}, J=10.6 \mathrm{~Hz}, 1 \mathrm{H}, \mathrm{OCHPh})$, 7.16-7.19 (m, 4H, Ar-H), 7.22-7.38 (m, 26H, Ar-H); ${ }^{13} \mathrm{C}$ NMR $\left(126 \mathrm{MHz}, \mathrm{CDCl}_{3}\right) \delta 56.3,66.6,68.3,68.8,73.5$, 73.6, 74.8, 75.0, 75.2, 75.3, 75.4, 77.8, 78.3, 78.9, 83.4, 85.1, 101,1 (C-1'), 102.4 (C-1), 127.5, 127.6, 127.65, $127.67,127.72,127.77,127.81,127.90,127.94,128.0$, $128.3,128.4,137.9,138.0,138.2,138.3,138.4$; FABHRMS $m / z$ calcd for $\mathrm{C}_{55} \mathrm{H}_{59} \mathrm{~N}_{3} \mathrm{O}_{10} \mathrm{Na}(\mathrm{M}+\mathrm{Na})^{+} 944.4098$, found 944.4072; Anal. calcd for: $\mathrm{C}_{55} \mathrm{H}_{59} \mathrm{~N}_{3} \mathrm{O}_{10}$ : C, 71.64; $\mathrm{H}$, $6.45 ; \mathrm{N}, 4.56$, found $\mathrm{C}, 71.58 ; \mathrm{H}, 6.49 ; \mathrm{N}, 4.61$. Data for $\alpha-$ anomer $(\mathbf{1 8} \alpha):^{12 \mathrm{~b}}$ TLC $R_{f}=0.66(2: 1$ hexane/AcOEt $) ;[\alpha]_{\mathrm{D}}{ }^{28}$ $+67.4^{\circ}\left(c 1.00, \mathrm{CHCl}_{3}\right)$; IR (film) 3030, 2918, 2864, 2104, 1496, 1454, 1359, 1211, 1126, 1055, $734 \mathrm{~cm}^{-1}$; ${ }^{1} \mathrm{H}$ NMR $\left(500 \mathrm{MHz}, \mathrm{CDCl}_{3}\right) \delta 3.26\left(\mathrm{~d}, J=1.6 \mathrm{~Hz}, 2 \mathrm{H}, \mathrm{H}-6^{\prime}\right), 3.34$ $\left(\mathrm{dd}, J=3.7,10.4 \mathrm{~Hz}, 1 \mathrm{H}, \mathrm{H}-2^{\prime}\right), 3.48(\mathrm{ddd}, J=2.0,3.7,9.4$ $\mathrm{Hz}, 1 \mathrm{H}, \mathrm{H}-5), 3.57\left(\mathrm{~s}, 3 \mathrm{H}, \mathrm{OCH}_{3}\right), 3.61(\mathrm{dd}, J=9.0,9.2 \mathrm{~Hz}$, 1H, H-3), 3.64-3.76 (m, 5H, H-2, H-4, H-6a, H-6b, H-4'), 3.91 (dd, $\left.J=8.9,10.4 \mathrm{~Hz}, 1 \mathrm{H}, \mathrm{H}-3^{\prime}\right), 3.98$ (m, 1H, H-5'), $4.28(\mathrm{~d}, J=12.0 \mathrm{~Hz}, 1 \mathrm{H}, \mathrm{OCHPh}), 4.38(\mathrm{~d}, J=7.4 \mathrm{~Hz}, 1 \mathrm{H}$, $\mathrm{H}-1), 4.43$ (d, $J=11.0 \mathrm{~Hz}, 1 \mathrm{H}, \mathrm{OC} H \mathrm{Ph}), 4.53$ (d, $J=12.0$ $\mathrm{Hz}, 1 \mathrm{H}, \mathrm{OCHPh}), 4.55$ (d, $J=12.2 \mathrm{~Hz}, 1 \mathrm{H}, \mathrm{OCHPh}), 4.56$ (d, $J=10.9 \mathrm{~Hz}, 1 \mathrm{H}, \mathrm{OCHPh}), 4.64(\mathrm{~d}, J=12.2 \mathrm{~Hz}, 1 \mathrm{H}$, $\mathrm{OCHPh}), 4.72$ (d, $J=10.8 \mathrm{~Hz}, 1 \mathrm{H}, \mathrm{OCHPh}), 4.74(\mathrm{~d}, J=$ $11.0 \mathrm{~Hz}, 1 \mathrm{H}, \mathrm{OCHPh}), 4.79$ (d, $J=10.9 \mathrm{~Hz}, 1 \mathrm{H}, \mathrm{OCHPh})$, $4.85(\mathrm{~d}, J=11.3 \mathrm{~Hz}, 1 \mathrm{H}, \mathrm{OCHPh}), 4.87(\mathrm{~d}, J=11.3 \mathrm{~Hz}, 1 \mathrm{H}$, OCHPh), $4.91(\mathrm{~d}, J=10.8 \mathrm{~Hz}, 1 \mathrm{H}, \mathrm{OCHPh}), 5.58(\mathrm{~d}, J=$ $\left.3.7 \mathrm{~Hz}, 1 \mathrm{H}, \mathrm{H}-1^{\prime}\right), 7.06-7.08$ (m, 4H, Ar-H), 7.12-7.18 (m, $3 \mathrm{H}, \mathrm{Ar}-\mathrm{H}), 7.22-7.36$ (m, 23H, Ar-H); ${ }^{13} \mathrm{C}$ NMR (100 MHz, $\left.\mathrm{CDCl}_{3}\right) \delta 57.2,63.3,67.6,68.5,70.4,73.4,73.5,74.8,74.9$, 75.3, 75.8, 76.3, 77.2, 78.1, 78.5, 80.0, 83.1, 96.7 (C-1'), 104.5 (C-1), 127.4, 127.50, 127.54, 127.6, 127.68, 127.73, $127.8,127.9,128.0,128.1,128.2,128.3,128.4,137.69$, 137.72, 137.8, 137.91, 137.94, 138.3; FAB-HRMS m/z calcd for $\mathrm{C}_{55} \mathrm{H}_{59} \mathrm{~N}_{3} \mathrm{O}_{10} \mathrm{Na} \quad(\mathrm{M}+\mathrm{Na})^{+}$944.4098, found 944.4110.

\subsubsection{Methyl 3-O-(2-azido-3,4,6-tri- $O$-benzyl-2-deoxy-D- glucopyranosyl)-2,4,6-tri- $O$-benzyl- $\alpha$-D-galactopyrano-} side (20). The glycosidation was performed according to the typical procedure $\left(1.5 \mathrm{~mL} \mathrm{EtCN},-78{ }^{\circ} \mathrm{C}, 2 \mathrm{~h}\right)$ employing diphenyl phosphate $\mathbf{2 a}(70.8 \mathrm{mg}, 0.10 \mathrm{mmol})$, alcohol 14 (51.1 mg, $0.11 \mathrm{mmol})$, and TMSOTf (1.0 $\mathrm{M}$ in $\left.\mathrm{CH}_{2} \mathrm{Cl}_{2}, 0.15 \mathrm{~mL}, 0.15 \mathrm{mmol}\right)$. An anomeric mixture of disaccharide $20(81.7 \mathrm{mg}, 89 \%, \alpha: \beta=1:>99)$ was obtained as a colorless oil from the crude product $(108.5 \mathrm{mg})$ after column chromatography (silica gel $6 \mathrm{~g}, 5: 1$ hexane/AcOEt). The anomeric ratio of the product was determined by HPLC analysis [eluent, 5:1 hexane/AcOEt; flow rate, 1.0 $\mathrm{mL} / \mathrm{min} ; t_{\mathrm{R}}(\alpha$-anomer $)=29.9 \mathrm{~min}, t_{\mathrm{R}}(\beta$-anomer $)=36.3$ $\min ]$. The $\alpha$ - and $\beta$-glycosides were separated by flash column chromatography with 20:1 toluene/AcOEt. Data for $\beta$-anomer (20 $\beta)$ : TLC $R_{f}=0.42$ (2:1 hexane/AcOEt), 0.28 $\left(10: 1\right.$ toluene/AcOEt); $[\alpha]_{\mathrm{D}}{ }^{16}-7.13^{\circ}$ (c 1.17, $\left.\mathrm{CHCl}_{3}\right)$; IR $\left(\mathrm{CHCl}_{3}\right)$ 3024, 2914, 2870, 2112, 1454, 1358, 1273, 1091 $\mathrm{cm}^{-1}$; ${ }^{1} \mathrm{H} \mathrm{NMR}\left(500 \mathrm{MHz}, \mathrm{CDCl}_{3}\right) \delta 3.32\left(\mathrm{~s}, 3 \mathrm{H}, \mathrm{OCH}_{3}\right)$, $3.38-3.43$ (m, 3H, H-2', H-4', H-5'), 3.48 (dd, $J=6.6,9.5$ $\mathrm{Hz}, 1 \mathrm{H}, \mathrm{H}-6 \mathrm{a}), 3.51$ (dd, $J=6.2,9.5 \mathrm{~Hz}, 1 \mathrm{H}, \mathrm{H}-6 \mathrm{~b})$, 3.66-3.71 (m, 2H, H-3', H-6'a), 3.74 (dd, $J=3.9,11.0 \mathrm{~Hz}$, $\left.1 \mathrm{H}, \mathrm{H}-6^{\prime} \mathrm{b}\right), 3.93$ (dd, $\left.J=6.2,6.6 \mathrm{~Hz}, 1 \mathrm{H}, \mathrm{H}-5\right), 4.00$ (brd, $J$ $=3.0 \mathrm{~Hz}, 1 \mathrm{H}, \mathrm{H}-4), 4.06(\mathrm{dd}, J=3.6,10.1 \mathrm{~Hz}, 1 \mathrm{H}, \mathrm{H}-2)$, $4.19(\mathrm{dd}, J=3.0,10.1 \mathrm{~Hz}, 1 \mathrm{H}, \mathrm{H}-3), 4.38$ (d, $J=11.8 \mathrm{~Hz}$, $1 \mathrm{H}, \mathrm{OC} H \mathrm{Ph}), 4.46(\mathrm{~d}, J=11.8 \mathrm{~Hz}, 1 \mathrm{H}, \mathrm{OC} H \mathrm{Ph}), 4.49$ (d, $J$ $=12.2 \mathrm{~Hz}, 1 \mathrm{H}$, OCHPh $), 4.57-4.61(\mathrm{~m}, 5 \mathrm{H}, \mathrm{H}-1$, $\mathrm{OCHPh} \times 4), 4.73\left(\mathrm{~m}, 1 \mathrm{H}, \mathrm{H}-1^{\prime}\right), 4.80(\mathrm{~d}, J=10.8 \mathrm{~Hz}, 1 \mathrm{H}$, $\mathrm{OC} H \mathrm{Ph}), 4.81(\mathrm{~d}, J=10.9 \mathrm{~Hz}, 1 \mathrm{H}, \mathrm{OCHPh}), 4.88(\mathrm{~d}, J=$ $10.9 \mathrm{~Hz}, 1 \mathrm{H}, \mathrm{OCHPh}), 4.90$ (d, $J=10.9 \mathrm{~Hz}, 1 \mathrm{H}, \mathrm{OC} H \mathrm{Ph})$, $4.95(\mathrm{~d}, J=11.4 \mathrm{~Hz}, 1 \mathrm{H}, \mathrm{OCHPh}), 7.18(\mathrm{~m}, 2 \mathrm{H}, \mathrm{Ar}-\mathrm{H})$, 7.20-7.38 (m, 26H, Ar-H), 7.42 (m, 2H, Ar-H); ${ }^{13} \mathrm{C}$ NMR $\left(126 \mathrm{MHz} \mathrm{CDCl}_{3}\right) \delta 55.3,67.1,68.5,69.1,69.2,73.4,73.5$, 73.6, 74.7, 75.05, 75.14, 75.5, 77.2, 77.4, 77.7, 83.0, 98.3 (C-1), $102.7\left(\mathrm{C}-1^{\prime}\right), 127.5,127.59,127.64,127.7,127.79$, $127.82,127.84,127.9,128.2$, 128.3, 128.35, 128.40, 128.42, $128.5,137.95,138.03,138.10,138.12,138.4,138.7$; FABHRMS $m / z$ calcd for $\mathrm{C}_{55} \mathrm{H}_{59} \mathrm{~N}_{3} \mathrm{O}_{10} \mathrm{Na}(\mathrm{M}+\mathrm{Na})^{+} 944.4098$, found 944.4136; Anal. calcd for: $\mathrm{C}_{55} \mathrm{H}_{59} \mathrm{~N}_{3} \mathrm{O}_{10}$ : C, 71.64; $\mathrm{H}$, 6.45; N, 4.56, found C, 71.67; H, 6.49; N 4.56. Data for $\alpha-$ anomer $(\mathbf{2 0} \alpha)$ : TLC $R_{f}=0.44(2: 1$ hexane/AcOEt $), 0.33$ $\left(10: 1\right.$ toluene/AcOEt); $[\alpha]_{\mathrm{D}}{ }^{24}+66.5^{\circ}\left(c \quad 0.95, \mathrm{CHCl}_{3}\right)$; IR $\left(\mathrm{CHCl}_{3}\right)$ 3022, 2914, 2870, 2112, 1454, 1358, 1209, 1091 $\mathrm{cm}^{-1}$; ${ }^{1} \mathrm{H}$ NMR $\left(500 \mathrm{MHz}, \mathrm{CDCl}_{3}\right) \delta 3.33\left(\mathrm{~s}, 3 \mathrm{H}, \mathrm{OCH}_{3}\right)$, 3.50-3.57 (m, 4H, H-6a, H-6b, H-2', H-6'a), 3.62 (dd, $J=$ 2.8, $\left.11.1 \mathrm{~Hz}, 1 \mathrm{H}, \mathrm{H}-6^{\prime} \mathrm{b}\right), 3.79$ (dd, $J=9.4,9.6 \mathrm{~Hz}, 1 \mathrm{H}, \mathrm{H}-$ $\left.4^{\prime}\right), 3.90(\mathrm{dd}, J=6.5,6.6 \mathrm{~Hz}, 1 \mathrm{H}, \mathrm{H}-5), 3.99-4.03(\mathrm{~m}, 2 \mathrm{H}$, H-2, H-4), 4.05 (dd, $\left.J=9.4,9.8 \mathrm{~Hz}, 1 \mathrm{H}, \mathrm{H}-33^{\prime}\right), 4.16-4.19$ (m, 2H, H-3, H-5'), 4.35 (d, $J=11.9 \mathrm{~Hz}, 1 \mathrm{H}, \mathrm{OCHPh}), 4.41$ $(\mathrm{d}, J=11.9 \mathrm{~Hz}, 1 \mathrm{H}, \mathrm{OCHPh}), 4.489(\mathrm{~d}, J=11.9 \mathrm{~Hz}, 1 \mathrm{H}$, OCHPh), 4.490 (d, $J=11.0 \mathrm{~Hz}, 1 \mathrm{H}, \mathrm{OCHPh}), 4.562$ (d, $J=$ 
$11.7 \mathrm{~Hz}, 1 \mathrm{H}, \mathrm{OCHPh}), 4.564(\mathrm{~d}, J=11.2 \mathrm{~Hz}, 1 \mathrm{H}, \mathrm{OC} H \mathrm{Ph})$, $4.60(\mathrm{~d}, J=11.9 \mathrm{~Hz}, 1 \mathrm{H}, \mathrm{OCHPh}), 4.70(\mathrm{~d}, J=3.6 \mathrm{~Hz}, 1 \mathrm{H}$, $\mathrm{H}-1), 4.72(\mathrm{~d}, J=11.7 \mathrm{~Hz}, 1 \mathrm{H}, \mathrm{OC} H \mathrm{Ph}), 4.77$ (d, $J=11.0$ $\mathrm{Hz}, 1 \mathrm{H}, \mathrm{OCHPh}), 4.82$ (d, $J=10.8 \mathrm{~Hz}, 1 \mathrm{H}, \mathrm{OCHPh}), 4.87$ $(\mathrm{d}, J=10.8 \mathrm{~Hz}, 1 \mathrm{H}, \mathrm{OCHPh}), 5.06(\mathrm{~d}, J=11.2 \mathrm{~Hz}, 1 \mathrm{H}$, OCHPh), $5.21\left(\mathrm{~d}, J=3.5 \mathrm{~Hz}, 1 \mathrm{H}, \mathrm{H}^{-1} 1^{\prime}\right), 7.11(\mathrm{~m}, 2 \mathrm{H}, \mathrm{Ar}-$ $\mathrm{H})$, 7.18-7.20 (m, 3H, Ar-H), 7.22-7.35 (m, 25H, Ar-H);

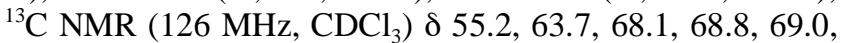
$70.5,73.3,73.4,74.4,74.7,74.8,75.1,75.3,78.3,80.2$, $94.7\left(\mathrm{C}-1^{\prime}\right), 98.4(\mathrm{C}-1), 127.5,127.55,127.62,127.71$, 127.74, 127.8, 128.0, 128.1, 128.2, 128.25, 128.28, 128.36, $128.39,137.87,137.92,138.1,138.3,138.6$; FAB-HRMS $m / z$ calcd for $\mathrm{C}_{55} \mathrm{H}_{59} \mathrm{~N}_{3} \mathrm{O}_{10} \mathrm{Na}(\mathrm{M}+\mathrm{Na})^{+}$944.4098, found 944.4097.

\subsubsection{Methyl 4- $O$-(2-azido-3,4,6-tri- $O$-benzyl-2-deoxy-D-} glucopyranosyl)-2,3,6-tri- $O$-benzyl- $\alpha$-D-galactopyrano-

side (21). The glycosidation was performed according to the typical procedure $\left(1.5 \mathrm{~mL}\right.$ EtCN, $\left.-78{ }^{\circ} \mathrm{C}, 2 \mathrm{~h}\right)$ employing diphenyl phosphate 2 a $(70.8 \mathrm{mg}, 0.10 \mathrm{mmol})$, alcohol 15 (51.1 mg, 0.11 mmol), and TMSOTf (1.0 M in $\left.\mathrm{CH}_{2} \mathrm{Cl}_{2}, 0.15 \mathrm{~mL}, 0.15 \mathrm{mmol}\right)$. An anomeric mixture of disaccharide 21 (82.5 mg, 90\%, $\alpha: \beta=5: 95)$ was obtained as a colorless oil from the crude product $(100.6 \mathrm{mg})$ after column chromatography (silica gel $8 \mathrm{~g}, 4: 1$ hexane/AcOEt). The anomeric ratio of the product was determined by HPLC analysis [eluent, 4:1 hexane/AcOEt; flow rate, 1.0 $\mathrm{mL} / \mathrm{min} ; t_{\mathrm{R}}(\alpha$-anomer $)=12.4 \mathrm{~min}, t_{\mathrm{R}}(\beta$-anomer $)=15.5$ min]. The $\alpha$ - and $\beta$-glycosides were separated by flash column chromatography with 15:1 toluene/AcOEt. Data for $\beta$-anomer (21 $\beta)$ : TLC $R_{f}=0.46(2: 1$ hexane/AcOEt), 0.34 $(10: 1$ toluene/AcOEt $) ;[\alpha]_{\mathrm{D}}{ }^{19}+3.32^{\circ}\left(c 0.74, \mathrm{CHCl}_{3}\right)$; IR $\left(\mathrm{CHCl}_{3}\right)$ 3020, 2930, 2868, 2114, 1454, 1358, 1277, 1089 $\mathrm{cm}^{-1}$; ${ }^{1} \mathrm{H}$ NMR $\left(500 \mathrm{MHz}, \mathrm{CDCl}_{3}\right) \delta 3.27$ (ddd, $J=1.9,3.9$, $\left.9.9 \mathrm{~Hz}, 1 \mathrm{H}, \mathrm{H}-5^{\prime}\right), 3.34$ (dd, $\left.J=8.8,9.8 \mathrm{~Hz}, 1 \mathrm{H}, \mathrm{H}-3^{\prime}\right), 3.37$ $\left(\mathrm{s}, 3 \mathrm{H}, \mathrm{OCH}_{3}\right), 3.40\left(\mathrm{dd}, J=7.6,9.8 \mathrm{~Hz}, 1 \mathrm{H}, \mathrm{H}-2^{\prime}\right)$, 3.58-3.65 (m, 4H, H-6a, H-4', H-6'a, H-6'b), 3.73 (dd, $J=$ 4.7, $10.5 \mathrm{~Hz}, 1 \mathrm{H}, \mathrm{H}-6 \mathrm{~b}), 3.90-3.94$ (m, 2H, H-3, H-5), 4.13 $(\mathrm{dd}, J=3.7,10.1 \mathrm{~Hz}, 1 \mathrm{H}, \mathrm{H}-2), 4.16(\mathrm{brd}, J=3.0 \mathrm{~Hz}, 1 \mathrm{H}$, $\mathrm{H}-4), 4.37$ (d, $J=12.0 \mathrm{~Hz}, 1 \mathrm{H}, \mathrm{OCHPh}), 4.44(\mathrm{~d}, J=12.0$ $\mathrm{Hz}, 1 \mathrm{H}, \mathrm{OCHPh}), 4.48$ (d, $J=12.1 \mathrm{~Hz}, 1 \mathrm{H}, \mathrm{OCHPh}), 4.52$ $(\mathrm{d}, J=12.1 \mathrm{~Hz}, 1 \mathrm{H}, \mathrm{OCHPh}), 4.54(\mathrm{~d}, J=12.1 \mathrm{~Hz}, 1 \mathrm{H}$, OCHPh), $4.64(\mathrm{~d}, J=3.7 \mathrm{~Hz}, 1 \mathrm{H}, \mathrm{H}-1), 4.67(\mathrm{~d}, J=12.1$ $\mathrm{Hz}, 1 \mathrm{H}, \mathrm{OCHPh}), 4.69$ (d, $J=12.1 \mathrm{~Hz}, 1 \mathrm{H}, \mathrm{OCHPh}), 4.73$ $\left(\mathrm{d}, J=7.6 \mathrm{~Hz}, 1 \mathrm{H}, \mathrm{H}-1^{\prime}\right), 4.77-4.80(\mathrm{~m}, 2 \mathrm{H}, \mathrm{OCHPh} \times 2)$, $4.84(\mathrm{~d}, J=12.1 \mathrm{~Hz}, 1 \mathrm{H}, \mathrm{OCHPh}), 4.91(\mathrm{~d}, J=10.8 \mathrm{~Hz}, 1 \mathrm{H}$, OCHPh), $4.92(\mathrm{~d}, J=12.1 \mathrm{~Hz}, 1 \mathrm{H}, \mathrm{OCHPh}), 7.15(\mathrm{~m}, 2 \mathrm{H}$, Ar-H), 7.24-7.37 (m, 28H, Ar-H); ${ }^{13} \mathrm{C}$ NMR (126 MHz, $\left.\mathrm{CDCl}_{3}\right) \delta 55.3,66.6,68.9,69.4,70.2,73.2,73.4,73.5,73.8$, 74.3, 74.8, 75.0, 75.5, 76.6, 77.7, 78.4, 83.1, 98.8 (C-1), $101.6\left(\mathrm{C}-1^{\prime}\right), 127.2,127.3,127.4,127.5,127.6,127.7$, $127.80,127.82,127.9,128.0,128.2,128.27,128.32,128.36$, $128.42,128.44,137.9,138.05,138.14,138.58,138.61$, 138.9; FAB-HRMS $m / z$ calcd for $\mathrm{C}_{55} \mathrm{H}_{59} \mathrm{~N}_{3} \mathrm{O}_{10} \mathrm{Na}(\mathrm{M}+\mathrm{Na})^{+}$ 944.4098, found 944.4102; Anal. calcd for: $\mathrm{C}_{55} \mathrm{H}_{59} \mathrm{~N}_{3} \mathrm{O}_{10}$ : C, 71.64; H, 6.45; N, 4.56, found C, 71.68; H, 6.55; N, 4.55. Data for $\alpha$-anomer $(\mathbf{2 1} \alpha)$ : TLC $R_{f}=0.43$ (2:1 hexane/ AcOEt), 0.40 (10:1 toluene/AcOEt); $[\alpha]_{\mathrm{D}}{ }^{18}+44.5^{\circ}$ (c 0.21 , $\left.\mathrm{CHCl}_{3}\right)$; IR $\left(\mathrm{CHCl}_{3}\right)$ 3018, 2930, 2870, 2114, 1454, 1358, $1277,1089 \mathrm{~cm}^{-1} ;{ }^{1} \mathrm{H}$ NMR $\left(500 \mathrm{MHz}, \mathrm{CDCl}_{3}\right) \delta 3.03(\mathrm{dd}, J$ $\left.=1.7,11.1 \mathrm{~Hz}, 1 \mathrm{H}, \mathrm{H}-6^{\prime} \mathrm{a}\right), 3.28(\mathrm{dd}, J=2.0,11.1 \mathrm{~Hz}, 1 \mathrm{H}$,
H-6'b), 3.35 (dd, $\left.J=3.6,9.2 \mathrm{~Hz}, 1 \mathrm{H}, \mathrm{H}-2^{\prime}\right), 3.36(\mathrm{~s}, 3 \mathrm{H}$, $\left.\mathrm{OCH}_{3}\right), 3.54(\mathrm{dd}, J=10.4,12.9 \mathrm{~Hz}, 1 \mathrm{H}, \mathrm{H}-5), 3.75$ (dd, $J=$ 9.4, $\left.10.1 \mathrm{~Hz}, 1 \mathrm{H}, \mathrm{H}-4^{\prime}\right)$, 3.83-3.92 (m, 5H, H-2, H-3, H-6a, H-6b, H-3'), 4.18 (d, $J=12.2 \mathrm{~Hz}, 1 \mathrm{H}, \mathrm{OCHPh}), 4.20$ (d, $J$ $=2.9 \mathrm{~Hz}, 1 \mathrm{H}, \mathrm{H}-4), 4.23(\mathrm{ddd}, J=1.7,2.0,10.1 \mathrm{~Hz}, 1 \mathrm{H}, \mathrm{H}-$ $\left.5^{\prime}\right), 4.43(\mathrm{~d}, J=12.2 \mathrm{~Hz}, 1 \mathrm{H}, \mathrm{OCHPh}), 4.46(\mathrm{~d}, J=12.2 \mathrm{~Hz}$, 1H, OCHPh), 4.52 (d, $J=11.8 \mathrm{~Hz}, 1 \mathrm{H}, \mathrm{OCHPh}), 4.55$ (d, $J$ $=11.8 \mathrm{~Hz}, 1 \mathrm{H}$, OCHPh $), 4.69-4.74(\mathrm{~m}, 4 \mathrm{H}, \mathrm{H}-1$, $\mathrm{OCHPh} \times 3), 4.77(\mathrm{~d}, J=11.9 \mathrm{~Hz}, 1 \mathrm{H}, \mathrm{OCHPh}), 4.80-4.82$ $(\mathrm{m}, 2 \mathrm{H}, \mathrm{OC} H \mathrm{Ph} \times 2), 4.86(\mathrm{~d}, J=10.6 \mathrm{~Hz}, 1 \mathrm{H}, \mathrm{OC} H \mathrm{Ph})$, $4.94\left(\mathrm{~d}, J=3.6 \mathrm{~Hz}, 1 \mathrm{H}, \mathrm{H}-1^{\prime}\right), 7.13(\mathrm{~m}, 2 \mathrm{H}, \mathrm{Ar}-\mathrm{H})$, 7.17-7.37 (m, 28H, Ar-H); ${ }^{13} \mathrm{C} \mathrm{NMR}\left(126 \mathrm{MHz}, \mathrm{CDCl}_{3}\right) \delta$ 55.4, 64.1, 67.3, 67.5, 68.9, 70.7, 73.16, 73.19, 73.3, 73.6, 74.8, 74.9, 75.27, 75.33, 78.2, 80.4, 98.5, 98.6, 127.4, 127.5, $127.6,127.66,127.73,127.8,127.95,127.99,128.03$, $128.26,128.31,128.33,128.4,128.5,137.6,137.9,138.1$, 138.2, 138.4, 138.7; FAB-HRMS $\mathrm{m} / \mathrm{z}$ calcd for $\mathrm{C}_{55} \mathrm{H}_{59} \mathrm{~N}_{3} \mathrm{O}_{10} \mathrm{Na}(\mathrm{M}+\mathrm{Na})^{+}$944.4098, found 944.4136.

\subsubsection{2-Azido-3,4,6-tri- $O$-benzyl-2-deoxy-D-glucopyran- osyl-( $1 \rightarrow 6)-2,3,4$-tri- $O$-benzoyl- $\alpha$-D-glucopyranosyl} $N, N, N^{\prime}, N^{\prime}$-tetramethylphosphorodiamidate (22). The glycosidation was performed according to the typical procedure $\left(1.5 \mathrm{~mL} \mathrm{EtCN},-78{ }^{\circ} \mathrm{C}, 2 \mathrm{~h}\right)$ employing diphenyl phosphate 2a (70.8 mg, $0.10 \mathrm{mmol})$, alcohol 16 (68.9 mg, $0.11 \mathrm{mmol})$, and TMSOTf (1.0 M in $\mathrm{CH}_{2} \mathrm{Cl}_{2}, 0.20 \mathrm{~mL}, 0.20$ $\mathrm{mmol})$. An anomeric mixture of disaccharide $22(95.0 \mathrm{mg}$, $88 \%, \alpha: \beta=7: 93)$ was obtained as a colorless oil from the crude product $(126.0 \mathrm{mg})$ after column chromatography (silica gel $8 \mathrm{~g}, 1: 2$ hexane/AcOEt). The anomeric ratio of the product was determined by ${ }^{1} \mathrm{H}$ NMR [integration of $\mathrm{H} 1$, $\beta$-anomer (4.27 ppm), $\alpha$-anomer (4.94 ppm)]. The $\alpha$ - and $\beta$-glycosides were separated by flash column chromatography with 1:2 hexane/AcOEt. Data for $\beta$-anomer (22 $\beta$ ): TLC $R_{f}=0.37(1: 3$ hexane/AcOEt $) ;[\alpha]_{\mathrm{D}}{ }^{16}+2.06^{\circ}(c$ 1.66, $\left.\mathrm{CHCl}_{3}\right)$; IR $\left(\mathrm{CHCl}_{3}\right)$ 3018, 2978, 2114, 1730, 1452, 1358, $1107,947 \mathrm{~cm}^{-1} ;{ }^{1} \mathrm{H}$ NMR $\left(500 \mathrm{MHz}, \mathrm{CDCl}_{3}\right) \delta 2.61\left(\mathrm{~d}, J_{\mathrm{H}-\mathrm{P}}\right.$ $\left.=10.4 \mathrm{~Hz}, 6 \mathrm{H}, \mathrm{N}\left(\mathrm{CH}_{3}\right)_{2}\right), 2.71\left(\mathrm{~d}, J_{\mathrm{H}-\mathrm{P}}=10.1 \mathrm{~Hz}, 6 \mathrm{H}\right.$, $\left.\mathrm{N}\left(\mathrm{CH}_{3}\right)_{2}\right), 3.34$ (dd, $\left.J=7.9,9.8 \mathrm{~Hz}, 1 \mathrm{H}, \mathrm{H}-2^{\prime}\right), 3.36$ (ddd, $J$ $\left.=2.2,4.1,9.8 \mathrm{~Hz}, 1 \mathrm{H}, \mathrm{H}-5^{\prime}\right), 3.44(\mathrm{dd}, J=8.9,9.8 \mathrm{~Hz}, 1 \mathrm{H}$, H-3'), 3.58 (dd, $\left.J=8.9,9.8 \mathrm{~Hz}, 1 \mathrm{H}, \mathrm{H}-4^{\prime}\right), 3.62(\mathrm{dd}, J=2.2$, $\left.11.2 \mathrm{~Hz}, 1 \mathrm{H}, \mathrm{H}-6^{\prime} \mathrm{a}\right), 3.65$ (dd, $J=4.1,11.2 \mathrm{~Hz}, 1 \mathrm{H}, \mathrm{H}-6^{\prime} \mathrm{b}$ ), $3.76(\mathrm{dd}, J=5.1,11.1 \mathrm{~Hz}, 1 \mathrm{H}, \mathrm{H}-6 \mathrm{a}), 4.14(\mathrm{dd}, J=2.5$, $11.1 \mathrm{~Hz}, 1 \mathrm{H}, \mathrm{H}-6 \mathrm{~b}), 4.27$ (d, $\left.J=7.9 \mathrm{~Hz}, 1 \mathrm{H}, \mathrm{H}-1^{\prime}\right), 4.45$ (d, $J=12.2 \mathrm{~Hz}, 1 \mathrm{H}, \mathrm{OCHPh}), 4.48(\mathrm{ddd}, J=2.5,5.1,10.1 \mathrm{~Hz}$, $1 \mathrm{H}, \mathrm{H}-5), 4.540$ (d, $J=12.2 \mathrm{~Hz}, 1 \mathrm{H}, \mathrm{OCHPh}), 4.541$ (d, $J=$ $10.9 \mathrm{~Hz}, 1 \mathrm{H}, \mathrm{OCHPh}), 4.78$ (d, $J=10.9 \mathrm{~Hz}, 1 \mathrm{H}, \mathrm{OCHPh})$, $4.79(\mathrm{~d}, J=10.8 \mathrm{~Hz}, 1 \mathrm{H}, \mathrm{OCHPh}), 4.89(\mathrm{~d}, J=10.8 \mathrm{~Hz}, 1 \mathrm{H}$, OCHPh), 5.39 (ddd, $\left.J=3.3,10.3,1.5\left(J_{\mathrm{H}-\mathrm{P}}\right) \mathrm{Hz}, 1 \mathrm{H}, \mathrm{H}-2\right)$, $5.72(\mathrm{dd}, J=9.7,10.2 \mathrm{~Hz}, 1 \mathrm{H}, \mathrm{H}-4), 6.15(\mathrm{dd}, J=3.3,8.1$ $\left.\left(J_{\mathrm{H}-\mathrm{P}}\right) \mathrm{Hz}, 1 \mathrm{H}, \mathrm{H}-1\right), 6.19(\mathrm{dd}, J=9.7,10.3 \mathrm{~Hz}, 1 \mathrm{H}, \mathrm{H}-3)$, $7.16(\mathrm{~m}, 2 \mathrm{H}, \mathrm{Ar}-\mathrm{H}), 7.25-7.52(\mathrm{~m}, 22 \mathrm{H}, \mathrm{Ar}-\mathrm{H}), 7.87(\mathrm{~m}$, 2H, Ar-H), 7.94-7.97 (m, 4H, Ar-H); ${ }^{13} \mathrm{C}$ NMR (126 MHz, $\left.\mathrm{CDCl}_{3}\right) \delta 36.4\left(\mathrm{~d}, J_{\mathrm{C}-\mathrm{P}}=3.8 \mathrm{~Hz}\right), 36.5\left(\mathrm{~d}, J_{\mathrm{C}-\mathrm{P}}=4.0 \mathrm{~Hz}\right)$, $66.3,68.2,68.4,69.2,70.0,70.8,71.5\left(\mathrm{~d}, J_{\mathrm{C}-\mathrm{P}}=6.4 \mathrm{~Hz}\right)$, $73.4,74.9,75.1,75.6,77.6,83.2,92.0\left(\mathrm{~d}, J_{\mathrm{C}-\mathrm{P}}=3.9 \mathrm{~Hz}, \mathrm{C}-\right.$ 1), $102.1\left(\mathrm{C}-1^{\prime}\right), 127.6,127.7,127.79,127.84,128.0$, $128.29,128.32,128.33,128.38,128.42,128.95,129.03$, $129.1,129.7,129.8,129.9,133.16,133.22,133.4,137.87$, $137.89,138.0,165.2,165.4,165.9 ;{ }^{31} \mathrm{P}$ NMR (109 MHz, $\left.\mathrm{C}_{6} \mathrm{D}_{6}\right) \delta 19.7$; FAB-HRMS $\mathrm{m} / \mathrm{z}$ calcd for $\mathrm{C}_{58} \mathrm{H}_{63} \mathrm{~N}_{5} \mathrm{O}_{14} \mathrm{P}$ 
$(\mathrm{M}+\mathrm{H})^{+}$1084.4109, found 1084.4150; Anal. calcd for: $\mathrm{C}_{58} \mathrm{H}_{62} \mathrm{~N}_{5} \mathrm{O}_{14} \mathrm{P}: \mathrm{C}, 64.26 ; \mathrm{H}, 5.76 ; \mathrm{N}, 6.46$, found $\mathrm{C}, 64.33$; H, 5.83; N, 6.41. Data for $\alpha$-anomer $(22 \alpha)$ : TLC $R_{f}=0.25$ $\left(1: 3\right.$ hexane/AcOEt); $\left.[\alpha]_{\mathrm{D}}{ }^{17}+84.8^{\circ}(c) 1.13, \mathrm{CHCl}_{3}\right)$; IR $\left(\mathrm{CHCl}_{3}\right)$ 3026, 2934, 2114, 1730, 1452, $1278 \mathrm{~cm}^{-1} ;{ }^{1} \mathrm{H}$ $\mathrm{NMR}\left(500 \mathrm{MHz}, \mathrm{CDCl}_{3}\right) \delta 2.60\left(\mathrm{~d}, J_{\mathrm{H}-\mathrm{P}}=10.1 \mathrm{~Hz}, 6 \mathrm{H}\right.$, $\left.\mathrm{N}\left(\mathrm{CH}_{3}\right)_{2}\right), 2.69\left(\mathrm{~d}, J_{\mathrm{H}-\mathrm{P}}=10.0 \mathrm{~Hz}, 6 \mathrm{H}, \mathrm{N}\left(\mathrm{CH}_{3}\right)_{2}\right), 3.35(\mathrm{dd}, J$ $\left.=3.6,10.2 \mathrm{~Hz}, 1 \mathrm{H}, \mathrm{H}-2^{\prime}\right), 3.45(\mathrm{dd}, J=1.4,10.9 \mathrm{~Hz}, 1 \mathrm{H}$, H-6'a), 3.58 (dd, $\left.J=3.2,10.9 \mathrm{~Hz}, 1 \mathrm{H}, \mathrm{H}-6^{\prime} \mathrm{b}\right), 3.65-3.72$ (m, 3H, H-6a, H-4', H-5'), 3.92 (dd, $J=5.0,11.3 \mathrm{~Hz}, 1 \mathrm{H}$, H-6b), 4.02 (dd, $\left.J=8.4,10.2 \mathrm{~Hz}, 1 \mathrm{H}, \mathrm{H}-3^{\prime}\right), 4.37$ (d, $J=$ $12.1 \mathrm{~Hz}, 1 \mathrm{H}, \mathrm{OC} H \mathrm{Ph}$ ), 4.48 (ddd, $J=1.9,5.0,10.3 \mathrm{~Hz}, 1 \mathrm{H}$, $\mathrm{H}-5), 4.50(\mathrm{~d}, J=11.1 \mathrm{~Hz}, 1 \mathrm{H}, \mathrm{OCHPh}), 4.51$ (d, $J=12.1$ $\mathrm{Hz}, 1 \mathrm{H}, \mathrm{OC} H \mathrm{Ph}), 4.80(\mathrm{~d}, J=11.1 \mathrm{~Hz}, 1 \mathrm{H}, \mathrm{OCHPh}), 4.86$ $(\mathrm{d}, J=11.3 \mathrm{~Hz}, 1 \mathrm{H}, \mathrm{OCHPh}), 4.88(\mathrm{~d}, J=11.3 \mathrm{~Hz}, 1 \mathrm{H}$, OCHPh), 4.94 (d, $\left.J=3.6 \mathrm{~Hz}, 1 \mathrm{H}, \mathrm{H}_{-1}{ }^{\prime}\right), 5.38$ (ddd, $J=3.4$, $\left.10.3,1.5\left(J_{\mathrm{H}-\mathrm{P}}\right) \mathrm{Hz}, 1 \mathrm{H}, \mathrm{H}-2\right), 5.72(\mathrm{dd}, J=9.8,10.3 \mathrm{~Hz}, 1 \mathrm{H}$, $\mathrm{H}-4), 6.13\left(\mathrm{dd}, J=3.4,8.1\left(J_{\mathrm{H}-\mathrm{P}}\right) \mathrm{Hz}, 1 \mathrm{H}, \mathrm{H}-1\right), 6.16(\mathrm{dd}, J$ $=9.8,10.3 \mathrm{~Hz}, 1 \mathrm{H}, \mathrm{H}-3), 7.18(\mathrm{~m}, 2 \mathrm{H}, \mathrm{Ar}-\mathrm{H}), 7.24-7.50(\mathrm{~m}$, 22H, Ar-H), 7.87 (m, 2H, Ar-H), 7.93-7.95 (m, 4H, Ar-H); ${ }^{13} \mathrm{C}$ NMR $\left(126 \mathrm{MHz}, \mathrm{CDCl}_{3}\right) \delta 36.4\left(\mathrm{~d}, J_{\mathrm{C}-\mathrm{P}}=3.5 \mathrm{~Hz}\right), 36.6$ $\left(\mathrm{d}, J_{\mathrm{C}-\mathrm{P}}=3.6 \mathrm{~Hz}\right), 63.4,66.7,68.1,68.7,70.1,70.5,70.8$, $71.5\left(\mathrm{~d}, J_{\mathrm{C}-\mathrm{P}}=6.5 \mathrm{~Hz}\right), 73.4,74.9,75.4,78.2,80.0,92.2(\mathrm{~d}$, $\left.J_{\mathrm{C}-\mathrm{P}}=3.9 \mathrm{~Hz}, \mathrm{C}-1\right), 98.4\left(\mathrm{C}-1^{\prime}\right), 127.6,127.7,127.8,128.1$, $128.29,128.33,128.34,128.38,128.44,128.9,129.0,129.1$, $129.7,129.8,129.9,133.2,133.3,133.4,137.8,138.0$, 138.2, 165.0, 165.4, 166.0; ${ }^{31} \mathrm{P}$ NMR (109 MHz, $\left.\mathrm{C}_{6} \mathrm{D}_{6}\right) \delta$ 19.4; FAB-HRMS $m / z$ calcd for $\mathrm{C}_{58} \mathrm{H}_{63} \mathrm{~N}_{5} \mathrm{O}_{14} \mathrm{P}(\mathrm{M}+\mathrm{H})^{+}$ 1084.4109 , found 1084.4100 .

\subsection{Glycosidations of 2-azido-3,4,6-tri- $O$-benzyl-2- deoxygalactosyl diphenyl phosphate $4 a$}

4.3.1. Methyl 6-O-(2-azido-3,4,6-tri- $O$-benzyl-2-deoxy-Dgalactopyranosyl)-2,3,4-tri- $O$-benzyl- $\alpha$-D-glucopyranoside (25). ${ }^{9}$ The glycosidation was performed according to the typical procedure $\left(1.5 \mathrm{~mL} \mathrm{EtCN},-78{ }^{\circ} \mathrm{C}, 0.2 \mathrm{~h}\right)$ employing diphenyl phosphate $4 \mathbf{a}(70.8 \mathrm{mg}, 0.10 \mathrm{mmol})$, alcohol 7 (51.1 mg, $0.11 \mathrm{mmol})$, and TMSOTf $(1.0 \mathrm{M}$ in $\left.\mathrm{CH}_{2} \mathrm{Cl}_{2}, 0.15 \mathrm{~mL}, 0.15 \mathrm{mmol}\right)$. An anomeric mixture of disaccharide 25 (79.4 mg, 86\%, $\alpha: \beta=4: 96)$ was obtained as a white solid from the crude product $(108.0 \mathrm{mg})$ after column chromatography (silica gel $7 \mathrm{~g}, 5: 1$ hexane/AcOEt), along with propionate $26(2.7 \mathrm{mg}, 5 \%)$ as a colorless oil. The anomeric ratio of $\mathbf{2 5}$ was determined by HPLC analysis [eluent, 5:1 hexane/AcOEt; flow rate, $1.0 \mathrm{~mL} / \mathrm{min}$; $t_{\mathrm{R}}(\alpha$-anomer $)=20.5 \mathrm{~min}, t_{\mathrm{R}}(\beta$-anomer $\left.)=27.2 \mathrm{~min}\right]$. The $\alpha$ - and $\beta$-glycosides were separated by flash column chromatography with $6: 1$ hexane/AcOEt. Data for $\beta$ anomer $(\mathbf{2 5} \beta):{ }^{9} \mathrm{mp}$ 93.5-94.5 ${ }^{\circ} \mathrm{C}$ (colorless needles from AcOEt-hexane); TLC $R_{f}=0.42(2: 1$ hexane/AcOEt $) ;[\alpha]_{\mathrm{D}}{ }^{23}$ $+0.79^{\circ}$ (c 1.00, $\mathrm{CHCl}_{3}$ ); IR (KBr) 3030, 2912, 2856, 2110, 1496, 1454, 1358, 1284, 1105, $1062 \mathrm{~cm}^{-1} ;{ }^{1} \mathrm{H}$ NMR (500 $\mathrm{MHz}, \mathrm{CDCl}_{3}$ ) $\delta 3.29$ (dd, $\left.J=2.8,10.4 \mathrm{~Hz}, 1 \mathrm{H}, \mathrm{H}-3^{\prime}\right), 3.36$ $\left(\mathrm{s}, 3 \mathrm{H}, \mathrm{OCH}_{3}\right), 3.44\left(\mathrm{dd}, J=6.1,7.7 \mathrm{~Hz}, 1 \mathrm{H}, \mathrm{H}-5^{\prime}\right)$, 3.51-3.55 (m, 3H, H-2, H-4, H-6'a), 3.61 (dd, $J=7.7,9.9$ $\left.\mathrm{Hz}, 1 \mathrm{H}, \mathrm{H}-6^{\prime} \mathrm{b}\right), 3.64$ (dd, $\left.J=5.0,10.9 \mathrm{~Hz}, 1 \mathrm{H}, \mathrm{H}-6 \mathrm{a}\right), 3.79$ (ddd, $J=1.8,5.0,10.1 \mathrm{~Hz}, 1 \mathrm{H}, \mathrm{H}-5), 3.85$ (dd, $J=8.2,10.4$ $\left.\mathrm{Hz}, 1 \mathrm{H}, \mathrm{H}-2^{\prime}\right), 3.87$ (m, 1H, H-4'), 3.98 (t, $J=9.3 \mathrm{~Hz}, 1 \mathrm{H}$, H-3), 4.07 (dd, $J=1.8,10.9 \mathrm{~Hz}, 1 \mathrm{H}, \mathrm{H}-6 \mathrm{~b}), 4.09$ (d, $J=8.2$ $\mathrm{Hz}, 1 \mathrm{H}, \mathrm{H}-1^{\prime}$ ), 4.40 (d, $\left.J=11.8 \mathrm{~Hz}, 1 \mathrm{H}, \mathrm{OCHPh}\right), 4.43$ (d,
$J=11.8 \mathrm{~Hz}, 1 \mathrm{H}, \mathrm{OCHPh}), 4.53(\mathrm{~d}, J=11.3 \mathrm{~Hz}, 1 \mathrm{H}$, OCHPh), $4.60(\mathrm{~d}, J=3.5 \mathrm{~Hz}, 1 \mathrm{H}, \mathrm{H}-1), 4.639(\mathrm{~d}, J=11.3$ $\mathrm{Hz}, 1 \mathrm{H}, \mathrm{OCHPh}), 4.640(\mathrm{~d}, J=12.2 \mathrm{~Hz}, 1 \mathrm{H}, \mathrm{OCHPh}), 4.66$ $(\mathrm{d}, J=11.7 \mathrm{~Hz}, 1 \mathrm{H}, \mathrm{OCHPh}), 4.70(\mathrm{~d}, J=11.7 \mathrm{~Hz}, 1 \mathrm{H}$, OCHPh), $4.77(\mathrm{~d}, J=12.2 \mathrm{~Hz}, 1 \mathrm{H}, \mathrm{OCHPh}), 4.80(\mathrm{~d}, J=$ $10.0 \mathrm{~Hz}, 1 \mathrm{H}, \mathrm{OCHPh}), 4.86(\mathrm{~d}, J=11.3 \mathrm{~Hz}, 1 \mathrm{H}, \mathrm{OCHPh})$, $4.90(\mathrm{~d}, J=11.1 \mathrm{~Hz}, 1 \mathrm{H}, \mathrm{OCHPh}), 4.97$ (d, $J=10.0 \mathrm{~Hz}, 1 \mathrm{H}$, $\mathrm{OCHPh}), 7.24-7.38$ (m, 30H, Ar-H); ${ }^{13} \mathrm{C}$ NMR (126 MHz, $\left.\mathrm{CDCl}_{3}\right) \delta 55.1,63.2,68.27,68.34,69.8,72.2,72.4,73.4$, 73.52, 73.53, 74.6, 74.8, 75.7, 77.8, 79.9, 80.9, 82.1, 98.0 (C-1), $102.5\left(\mathrm{C}-1^{\prime}\right), 127.5,127.6,127.8,127.85,127.86$, $128.0,128.05,128.13,128.2,128.3,128.42,128.44,128.5$, 137.6, 137.8, 138.2, 138.4, 138.5, 138.8; FAB-HRMS $\mathrm{m} / \mathrm{z}$ calcd for $\mathrm{C}_{55} \mathrm{H}_{59} \mathrm{~N}_{3} \mathrm{O}_{10} \mathrm{Na} \quad(\mathrm{M}+\mathrm{Na})^{+}$944.4098, found 944.4093; Anal. calcd for: $\mathrm{C}_{55} \mathrm{H}_{59} \mathrm{~N}_{3} \mathrm{O}_{10}$ : C, 71.64; $\mathrm{H}, 6.45$; $\mathrm{N}, 4.56$, found $\mathrm{C}, 71.62 ; \mathrm{H}, 6.51 ; \mathrm{N}$ 4.55. Data for $\alpha-$ anomer $(\mathbf{2 5} \alpha):{ }^{9}$ TLC $R_{f}=0.48(2: 1$ hexane/AcOEt $) ;[\alpha]_{\mathrm{D}}{ }^{21}$ $+83.3^{\circ}$ (c 1.00, $\mathrm{CHCl}_{3}$ ); IR (film) 3030, 2916, 2108, 1496, 1454, 1358, 1259, 1159, $1095 \mathrm{~cm}^{-1}$; ${ }^{1} \mathrm{H}$ NMR $(500 \mathrm{MHz}$, $\left.\mathrm{CDCl}_{3}\right) \delta 3.33\left(\mathrm{~s}, 3 \mathrm{H}, \mathrm{OCH}_{3}\right), 3.49(\mathrm{dd}, J=6.1,9.2 \mathrm{~Hz}, 1 \mathrm{H}$, H-6'a), 3.51 (dd, $J=9.0,9.9 \mathrm{~Hz}, 1 \mathrm{H}, \mathrm{H}-4), 3.53$ (dd, $J=$ $3.6,9.6 \mathrm{~Hz}, 1 \mathrm{H}, \mathrm{H}-2), 3.56$ (dd, $\left.J=7.9,9.2 \mathrm{~Hz}, 1 \mathrm{H}, \mathrm{H}-6^{\prime} \mathrm{b}\right)$, $3.69(\mathrm{dd}, J=1.2,11.2 \mathrm{~Hz}, 1 \mathrm{H}, \mathrm{H}-6 \mathrm{a}), 3.75$ (ddd, $J=1.2$, 4.9, $9.9 \mathrm{~Hz}, 1 \mathrm{H}, \mathrm{H}-5), 3.80$ (dd, $J=4.9,11.2 \mathrm{~Hz}, 1 \mathrm{H}, \mathrm{H}-6 \mathrm{~b})$, $3.83\left(\mathrm{dd}, J=3.5,10.7 \mathrm{~Hz}, 1 \mathrm{H}, \mathrm{H}-2^{\prime}\right), 3.89(\mathrm{dd}, J=2.5,10.7$ $\left.\mathrm{Hz}, 1 \mathrm{H}, \mathrm{H}-3^{\prime}\right), 3.93$ (dd, $\left.J=6.1,7.9 \mathrm{~Hz}, 1 \mathrm{H}, \mathrm{H}-5^{\prime}\right), 3.992$ (br, 1H, H-4'), 3.994 (dd, $J=9.0,9.6 \mathrm{~Hz}, 1 \mathrm{H}, \mathrm{H}-3$ ), 4.37 (d, $J=11.8 \mathrm{~Hz}, 1 \mathrm{H}, \mathrm{OCHPh}), 4.44(\mathrm{~d}, J=11.8 \mathrm{~Hz}, 1 \mathrm{H}$, $\mathrm{OCHPh}), 4.53(\mathrm{~d}, J=11.3 \mathrm{~Hz}, 1 \mathrm{H}, \mathrm{OCHPh}), 4.56(\mathrm{~d}, J=$ $11.0 \mathrm{~Hz}, 1 \mathrm{H}, \mathrm{OCHPh}), 4.58$ (d, $J=3.6 \mathrm{~Hz}, 1 \mathrm{H}, \mathrm{H}-1), 4.649$ $(\mathrm{d}, J=11.4 \mathrm{~Hz}, 1 \mathrm{H}, \mathrm{OCHPh}), 4.654(\mathrm{~d}, J=12.0 \mathrm{~Hz}, 1 \mathrm{H}$, OCHPh), $4.71(\mathrm{~d}, J=11.4 \mathrm{~Hz}, 1 \mathrm{H}, \mathrm{OC} H \mathrm{Ph}), 4.78(\mathrm{~d}, J=$ $12.0 \mathrm{~Hz}, 1 \mathrm{H}, \mathrm{OCHPh}), 4.80$ (d, $J=10.8 \mathrm{~Hz}, 1 \mathrm{H}, \mathrm{OCHPh})$, $4.87(\mathrm{~d}, J=11.3 \mathrm{~Hz}, 1 \mathrm{H}, \mathrm{OCHPh}), 4.88(\mathrm{~d}, J=11.0 \mathrm{~Hz}, 1 \mathrm{H}$, OCHPh), $4.980(\mathrm{~d}, J=10.8 \mathrm{~Hz}, 1 \mathrm{H}, \mathrm{OC} H \mathrm{Ph}), 4.982(\mathrm{~d}, J=$ $\left.3.5 \mathrm{~Hz}, 1 \mathrm{H}, \mathrm{H}-1^{\prime}\right), 7.22-7.39(\mathrm{~m}, 30 \mathrm{H}, \mathrm{Ar}-\mathrm{H}) ;{ }^{13} \mathrm{C}$ NMR $\left(126 \mathrm{MHz} \mathrm{CDCl}_{3}\right) \delta 55.0,59.8,66.7,68.6,69.6,69.9,72.0$, 73.35, 73.37, 73.39, 74.8, 74.9, 75.7, 76.6, 77.9, 80.0, 82.0, $97.9,98.6,127.6,127.65,127.66,127.71,127.72,127.8$, $127.87,127.90,128.0,128.06,128.07,128.2,128.36$, $128.40,128.5,137.5,137.9,138.1,138.27,138.29,138.8$; FAB-HRMS $m / z$ calcd for $\mathrm{C}_{55} \mathrm{H}_{59} \mathrm{~N}_{3} \mathrm{O}_{10} \mathrm{Na} \quad(\mathrm{M}+\mathrm{Na})^{+}$ 944.4098, found 944.4079 .

Data for methyl 2,3,4-tri- $O$-benzyl-6- $O$-propionyl- $\alpha$-Dglucopyranoside (26): TLC $R_{f}=0.45$ (2:1 hexane/AcOEt); $[\alpha]_{\mathrm{D}}^{25}+27.1^{\circ}\left(c 1.00, \mathrm{CHCl}_{3}\right)$; IR (film) 3030, 2918, 1738, 1454, 1190, $1072 \mathrm{~cm}^{-1} ;{ }^{1} \mathrm{H}$ NMR $\left(500 \mathrm{MHz}, \mathrm{CDCl}_{3}\right) \delta 1.11$ (t, $\left.J=7.7 \mathrm{~Hz}, 3 \mathrm{H}, \mathrm{CH}_{2} \mathrm{CH}_{3}\right), 2.30\left(\mathrm{~m}, 2 \mathrm{H}, \mathrm{CH}_{2} \mathrm{CH}_{3}\right), 3.36$ (s, $\left.3 \mathrm{H}, \mathrm{OCH}_{3}\right), 3.47(\mathrm{dd}, J=8.9,10.1 \mathrm{~Hz}, 1 \mathrm{H}, \mathrm{H}-4), 3.53(\mathrm{dd}$, $J=3.5,9.6 \mathrm{~Hz}, 1 \mathrm{H}, \mathrm{H}-2), 3.82(\mathrm{ddd}, J=3.0,3.9,10.1 \mathrm{~Hz}$, $1 \mathrm{H}, \mathrm{H}-5), 4.01$ (dd, $J=8.9,9.6 \mathrm{~Hz}, 1 \mathrm{H}, \mathrm{H}-3), 4.26$ (dd, $J=$ $3.9,12.0 \mathrm{~Hz}, 1 \mathrm{H}, \mathrm{H}-6 \mathrm{a}), 4.29$ (dd, $J=3.0,12.0 \mathrm{~Hz}, 1 \mathrm{H}, \mathrm{H}-$ $6 \mathrm{~b}), 4.56(\mathrm{~d}, J=10.8 \mathrm{~Hz}, 1 \mathrm{H}, \mathrm{OCHPh}), 4.60(\mathrm{~d}, J=3.5 \mathrm{~Hz}$, $1 \mathrm{H}, \mathrm{H}-1), 4.66$ (d, $J=12.1 \mathrm{~Hz}, 1 \mathrm{H}, \mathrm{OCHPh}), 4.79$ (d, $J=$ $12.1 \mathrm{~Hz}, 1 \mathrm{H}, \mathrm{OCHPh}), 4.83$ (d, $J=10.8 \mathrm{~Hz}, 1 \mathrm{H}, \mathrm{OCHPh})$, $4.88(\mathrm{~d}, J=10.8 \mathrm{~Hz}, 1 \mathrm{H}, \mathrm{OCHPh}), 5.00(\mathrm{~d}, J=10.8 \mathrm{~Hz}, 1 \mathrm{H}$, OCHPh), 7.26-7.36 (m, 15H, Ar-H); ${ }^{13} \mathrm{C}$ NMR (126 MHz, $\left.\mathrm{CDCl}_{3}\right) \delta$ 9.0, 27.3, 55.1, 62.8, 68.6, 73.3, 75.0, 75.7, 77.4, 79.9, 82.0, 97.9 (C-1), 127.6, 127.8, 127.87, 127.92, 127.94, $128.0,128.3,128.38,128.39,137.8,138.0,138.5,174.0$; 
FAB-HRMS $\mathrm{m} / \mathrm{z}$ calcd for $\mathrm{C}_{31} \mathrm{H}_{36} \mathrm{O}_{7} \mathrm{Na}(\mathrm{M}+\mathrm{Na})^{+}$543.2359, found 543.2333; Anal. calcd for: $\mathrm{C}_{31} \mathrm{H}_{36} \mathrm{O}_{7}: \mathrm{C}, 71.52 ; \mathrm{H}$ 6.97, found C, 71.40; H, 6.93.

4.3.2. Methyl 4- $O$-(2-azido-3,4,6-tri- $O$-benzyl-2-deoxy-Dgalactopyranosyl)-2,3,6-tri- $O$-benzyl- $\alpha$-D-glucopyranoside (27). ${ }^{10}$ The glycosidation was performed according to the typical procedure $\left(1.5 \mathrm{~mL} \mathrm{EtCN},-78{ }^{\circ} \mathrm{C}, 0.5 \mathrm{~h}\right)$ employing diphenyl phosphate $4 \mathbf{a}(70.8 \mathrm{mg}, 0.10 \mathrm{mmol})$, alcohol 8 (51.1 mg, 0.11 mmol), and TMSOTf (1.0 $\mathrm{M}$ in $\left.\mathrm{CH}_{2} \mathrm{Cl}_{2}, 0.15 \mathrm{~mL}, 0.15 \mathrm{mmol}\right)$. An anomeric mixture of disaccharide $27(82.8 \mathrm{mg}, 90 \%, \alpha: \beta=4: 96)$ was obtained as a colorless oil from the crude product $(110.6 \mathrm{mg})$ after column chromatography (silica gel 5 g, 5:1 hexane/AcOEt). The anomeric ratio of the product was determined by HPLC analysis [eluent, 5:1 hexane/AcOEt; flow rate, 1.0 $\mathrm{mL} / \mathrm{min} ; t_{\mathrm{R}}(\alpha$-anomer $)=15.3 \mathrm{~min}, t_{\mathrm{R}}(\beta$-anomer $)=22.8$ $\min ]$. The $\alpha$ - and $\beta$-glycosides were separated by flash column chromatography with 6:1 hexane/AcOEt. Data for $\beta$-anomer $(\mathbf{2 7} \beta):^{10}$ TLC $R_{f}=0.49$ (2:1 hexane/AcOEt); $[\alpha]_{\mathrm{D}}{ }^{25}-3.12^{\circ}$ (c 2.51, $\mathrm{CHCl}_{3}$ ); IR (film) 3030, 2868, 2112, 1496, 1454, 1361, $1099 \mathrm{~cm}^{-1} ;{ }^{1} \mathrm{H}$ NMR $\left(500 \mathrm{MHz}, \mathrm{CDCl}_{3}\right)$

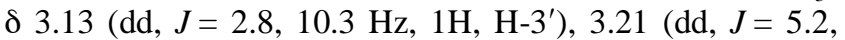
$\left.8.2 \mathrm{~Hz}, 1 \mathrm{H}, \mathrm{H}-5^{\prime}\right), 3.30$ (dd, $\left.J=5.2,9.2 \mathrm{~Hz}, 1 \mathrm{H}, \mathrm{H}-6^{\prime} \mathrm{a}\right)$, $3.37\left(\mathrm{~s}, 3 \mathrm{H}, \mathrm{OCH}_{3}\right), 3.47$ (dd, $\left.J=3.7,9.4 \mathrm{~Hz}, 1 \mathrm{H}, \mathrm{H}-2\right)$, $3.48\left(\mathrm{dd}, J=8.2,9.2 \mathrm{~Hz}, 1 \mathrm{H}, \mathrm{H}-6^{\prime} \mathrm{b}\right), 3.70$ (dd, $J=1.5,10.9$ $\mathrm{Hz}, 1 \mathrm{H}, \mathrm{H}-6 \mathrm{a}), 3.74$ (dd, $J=8.1,10.3 \mathrm{~Hz}, 1 \mathrm{H}, \mathrm{H}-2^{\prime}$ ), 3.76 (m, 1H, H-5), 3.849 (dd, $J=8.9,9.4 \mathrm{~Hz}, 1 \mathrm{H}, \mathrm{H}-4), 3.852$ (brd, $\left.J=2.8 \mathrm{~Hz}, 1 \mathrm{H}, \mathrm{H}-4^{\prime}\right), 3.91(\mathrm{dd}, J=9.3,9.4 \mathrm{~Hz}, 1 \mathrm{H}$, $\mathrm{H}-3), 3.94$ (dd, $J=3.2,10.9 \mathrm{~Hz}, 1 \mathrm{H}, \mathrm{H}-6 \mathrm{~b}), 4.14$ (d, $J=8.1$ $\left.\mathrm{Hz}, 1 \mathrm{H}, \mathrm{H}-1^{\prime}\right), 4.22$ (d, $\left.J=11.8 \mathrm{~Hz}, 1 \mathrm{H}, \mathrm{OCHPh}\right), 4.33$ (d, $J=11.8 \mathrm{~Hz}, 1 \mathrm{H}, \mathrm{OCHPh}), 4.43(\mathrm{~d}, J=12.0 \mathrm{~Hz}, 1 \mathrm{H}$, OCHPh), $4.50(\mathrm{~d}, J=11.3 \mathrm{~Hz}, 1 \mathrm{H}, \mathrm{OCHPh}), 4.58(\mathrm{~d}, J=$ $3.7 \mathrm{~Hz}, 1 \mathrm{H}, \mathrm{H}-1), 4.61$ (d, $J=11.7 \mathrm{~Hz}, 1 \mathrm{H}, \mathrm{OCHPh}), 4.62$ $(\mathrm{d}, J=12.1 \mathrm{~Hz}, 1 \mathrm{H}, \mathrm{OCHPh}), 4.66(\mathrm{~d}, J=12.0 \mathrm{~Hz}, 1 \mathrm{H}$, OCHPh), $4.68(\mathrm{~d}, J=11.7 \mathrm{~Hz}, 1 \mathrm{H}, \mathrm{OC} H \mathrm{Ph}), 4.74(\mathrm{~d}, J=$ $10.7 \mathrm{~Hz}, 1 \mathrm{H}, \mathrm{OC} H \mathrm{Ph}), 4.80$ (d, $J=12.1 \mathrm{~Hz}, 1 \mathrm{H}, \mathrm{OCHPh})$, $4.88(\mathrm{~d}, J=11.3 \mathrm{~Hz}, 1 \mathrm{H}, \mathrm{OCHPh}), 4.96(\mathrm{~d}, J=10.7 \mathrm{~Hz}, 1 \mathrm{H}$, $\mathrm{OCHPh}), 7.13-7.38(\mathrm{~m}, 30 \mathrm{H}, \mathrm{Ar}-\mathrm{H}) ;{ }^{13} \mathrm{C}$ NMR $(126 \mathrm{MHz}$, $\left.\mathrm{CDCl}_{3}\right) \delta 55.2,63.8,67.9,68.3,69.7,72.1,73.2,73.3,73.4$, 73.6, 74.7, 75.4, 76.7, 79.1, 80.1, 81.0, 98.3 (C-1), 101.2 $\left(\mathrm{C}-1^{\prime}\right), \quad 127.5,127.62,127.64,127.68,127.71,127.76$, $127.78,127.83,127.86,127.91,127.93,128.0,128.2$, 128.32 , 128.34, 128.4, 137.6, 138.0, 138.4, 138.6, 139.4; FAB-HRMS $m / z$ calcd for $\mathrm{C}_{55} \mathrm{H}_{60} \mathrm{~N}_{3} \mathrm{O}_{10}(\mathrm{M}+\mathrm{H})^{+} 922.4278$, found 922.4290; Anal. calcd for: $\mathrm{C}_{55} \mathrm{H}_{59} \mathrm{~N}_{3} \mathrm{O}_{10}$ : C, 71.64; $\mathrm{H}$, $6.45 ; \mathrm{N}, 4.56$, found $\mathrm{C}, 71.42 ; \mathrm{H}, 6.54 ; \mathrm{N}, 4.52$. Data for $\alpha-$ anomer $(27 \alpha):{ }^{10}$ TLC $R_{f}=0.55(2: 1$ hexane/AcOEt $) ;[\alpha]_{\mathrm{D}}{ }^{23}$ $+47.5^{\circ}\left(c 0.35, \mathrm{CHCl}_{3}\right)$; IR (film) $3030,2868,2112,1496$, 1454, 1361, $1099 \mathrm{~cm}^{-1} ;{ }^{1} \mathrm{H}$ NMR $\left(500 \mathrm{MHz}, \mathrm{CDCl}_{3}\right) \delta 3.38$ $\left(\mathrm{s}, 3 \mathrm{H}, \mathrm{OCH}_{3}\right), 3.39(\mathrm{~m}, 1 \mathrm{H}, \mathrm{H}-6$ 'a), 3.47 (dd, $J=8.0,8.6$ $\left.\mathrm{Hz}, 1 \mathrm{H}, \mathrm{H}-6^{\prime} \mathrm{b}\right), 3.55$ (dd, $\left.J=3.5,9.6 \mathrm{~Hz}, 1 \mathrm{H}, \mathrm{H}-2\right), 3.63$ (dd, $J=4.0,11.1 \mathrm{~Hz}, 1 \mathrm{H}, \mathrm{H}-6 \mathrm{a}), 3.66$ (dd, $J=2.2,11.1 \mathrm{~Hz}$, 1H, H-6b), 3.76-3.85 (m, 5H, H-4, H-5, H-2', H-3', H-5'), 3.96 (brs, $1 \mathrm{H}, \mathrm{H}-4^{\prime}$ ), 4.05 (dd, $J=8.4,9.6 \mathrm{~Hz}, 1 \mathrm{H}, \mathrm{H}-3$ ), $4.23(\mathrm{~d}, J=11.7 \mathrm{~Hz}, 1 \mathrm{H}, \mathrm{OCHPh}), 4.30(\mathrm{~d}, J=11.7 \mathrm{~Hz}, 1 \mathrm{H}$, OCHPh), 4.43 (d, $J=12.2 \mathrm{~Hz}, 1 \mathrm{H}, \mathrm{OCHPh}), 4.49$ (d, $J=$ $11.3 \mathrm{~Hz}, 1 \mathrm{H}, \mathrm{OCHPh}), 4.56$ (d, $J=12.2 \mathrm{~Hz}, 1 \mathrm{H}, \mathrm{OC} H \mathrm{Ph})$, $4.58(\mathrm{~d}, J=3.5 \mathrm{~Hz}, 1 \mathrm{H}, \mathrm{H}-1), 4.60(\mathrm{~d}, J=11.2 \mathrm{~Hz}, 1 \mathrm{H}$, OCHPh), $4.61(\mathrm{~d}, J=12.0 \mathrm{~Hz}, 1 \mathrm{H}, \mathrm{OCHPh}), 4.66(\mathrm{~d}, J=$ $11.2 \mathrm{~Hz}, 1 \mathrm{H}, \mathrm{OCHPh}), 4.75$ (d, $J=12.0 \mathrm{~Hz}, 1 \mathrm{H}, \mathrm{OCHPh})$, $4.81(\mathrm{~d}, J=11.3 \mathrm{~Hz}, 1 \mathrm{H}, \mathrm{OCHPh}), 4.87$ (d, $J=10.6 \mathrm{~Hz}, 1 \mathrm{H}$, OCHPh), $5.06(\mathrm{~d}, J=10.6 \mathrm{~Hz}, 1 \mathrm{H}, \mathrm{OCHPh}), 5.70(\mathrm{~d}, J=$ $\left.2.8 \mathrm{~Hz}, 1 \mathrm{H}, \mathrm{H}-1^{\prime}\right), 7.20-7.39$ (m, 30H, Ar-H); ${ }^{13} \mathrm{C}$ NMR $\left(126 \mathrm{MHz}, \mathrm{CDCl}_{3}\right) \delta 55.3,59.5,68.5,69.5,69.7,70.0,72.1$, $73.0,73.1,73.3,73.5,73.7,74.8,75.0,80.4,81.9,97.7$, $98.1,127.39,127.42,127.67,127.73,127.8,127.9,128.0$, $128.16,128.21,128.24,128.3,128.35,128.37,128.47$, $128.49,137.6,137.8,138.0,138.2,138.4,138.6$; FABHRMS $m / z$ calcd for $\mathrm{C}_{55} \mathrm{H}_{60} \mathrm{~N}_{3} \mathrm{O}_{10}(\mathrm{M}+\mathrm{H})^{+} 922.4278$, found 922.4301

\subsubsection{Methyl 4-O-(2-azido-3,4,6-tri- $O$-benzyl-2-deoxy-D- galactopyranosyl)-2,3- $O$-isopropylidene- $\alpha$-L-rhamno-}

pyranoside (28). The glycosidation was performed according to the typical procedure $\left(1.5 \mathrm{~mL} \mathrm{EtCN},-78^{\circ} \mathrm{C}\right.$, $0.3 \mathrm{~h}$ ) employing diphenyl phosphate $4 \mathbf{a}(70.8 \mathrm{mg}, 0.10$ mmol), alcohol 23 (24.0 mg, $0.11 \mathrm{mmol})$, and TMSOTf (1.0 $\mathrm{M}$ in $\mathrm{CH}_{2} \mathrm{Cl}_{2}, 0.15 \mathrm{~mL}, 0.15 \mathrm{mmol}$ ). An anomeric mixture of disaccharide 28 (55.0 mg, 81\%, $\alpha: \beta=6: 94)$ was obtained as a colorless oil from the crude product $(82.5 \mathrm{mg})$ after column chromatography (silica gel $7 \mathrm{~g}, 7: 1$ hexane/ AcOEt). The anomeric ratio of the product was determined by HPLC analysis [eluent, 5:1 hexane/THF; flow rate, 1.0 $\mathrm{mL} / \mathrm{min} ; t_{\mathrm{R}}(\alpha$-anomer $)=6.3 \mathrm{~min}, t_{\mathrm{R}}(\beta$-anomer $\left.)=7.4 \mathrm{~min}\right]$. The $\alpha$ - and $\beta$-glycosides were separated by flash column chromatography with $8: 1$ hexane/AcOEt. Data for $\beta$ anomer (28 $\beta)$ : TLC $R_{f}=0.57\left(2: 1\right.$ hexane/AcOEt); $[\alpha]_{\mathrm{D}}{ }^{24}$ $-33.8^{\circ}$ (c 1.47, $\mathrm{CHCl}_{3}$ ); IR (film) 2934, 2112, 1454, 1367, 1221, 1091, $1022 \mathrm{~cm}^{-1} ;{ }^{1} \mathrm{H}$ NMR $\left(500 \mathrm{MHz}, \mathrm{CDCl}_{3}\right) \delta 1.28$ $(\mathrm{d}, J=5.6 \mathrm{~Hz}, 3 \mathrm{H}, \mathrm{H}-6), 1.33\left(\mathrm{~s}, 3 \mathrm{H}, \mathrm{CCH}_{3}\right), 1.44(\mathrm{~s}, 3 \mathrm{H}$, $\left.\mathrm{CCH}_{3}\right), 3.34\left(\mathrm{dd}, J=3.0,10.4 \mathrm{~Hz}, 1 \mathrm{H}, \mathrm{H}-3^{\prime}\right), 3.36(\mathrm{~s}, 3 \mathrm{H}$, $\left.\mathrm{OCH}_{3}\right), 3.47\left(\mathrm{~m}, 1 \mathrm{H}, \mathrm{H}-5^{\prime}\right), 3.53(\mathrm{dd}, J=5.3,9.1 \mathrm{~Hz}, 1 \mathrm{H}$, H-6'a), 3.60-3.66 (m, 3H, H-4, H-5, H-6'b), 3.73 (dd, $J=$ 8.1, $\left.10.4 \mathrm{~Hz}, 1 \mathrm{H}, \mathrm{H}-2^{\prime}\right), 3.86$ (d, $\left.J=3.0 \mathrm{~Hz}, 1 \mathrm{H}, \mathrm{H}-4^{\prime}\right), 4.09$ $(\mathrm{d}, J=5.6 \mathrm{~Hz}, 1 \mathrm{H}, \mathrm{H}-2), 4.25(\mathrm{dd}, J=5.6,5.8 \mathrm{~Hz}, 1 \mathrm{H}, \mathrm{H}-3)$, $4.42(\mathrm{~d}, J=11.9 \mathrm{~Hz}, 1 \mathrm{H}, \mathrm{OCHPh}), 4.45(\mathrm{~d}, J=11.9 \mathrm{~Hz}, 1 \mathrm{H}$, OCHPh), $4.57(\mathrm{~d}, J=11.4 \mathrm{~Hz}, 1 \mathrm{H}, \mathrm{OCHPh}), 4.67$ (d, $J=$ $12.6 \mathrm{~Hz}, 1 \mathrm{H}, \mathrm{OCHPh}), 4.70$ (d, $J=12.6 \mathrm{~Hz}, 1 \mathrm{H}, \mathrm{OCHPh})$, $4.71\left(\mathrm{~d}, J=8.1 \mathrm{~Hz}, 1 \mathrm{H}, \mathrm{H}^{\prime} 1^{\prime}\right), 4.84$ (s, 1H, H-1), 4.89 (d, $J$ $=11.4 \mathrm{~Hz}, 1 \mathrm{H}, \mathrm{OCHPh}), 7.25-7.39(\mathrm{~m}, 15 \mathrm{H}, \mathrm{Ar}-\mathrm{H}) ;{ }^{13} \mathrm{C}$ NMR $\left(126 \mathrm{MHz}, \mathrm{CDCl}_{3}\right) \delta 17.7,26.4,27.8,54.8,63.4$, 64.1, 68.4, 72.6, 73.48, 73.50, 74.7, 76.0, 78.2, 78.5, 80.7, $97.9(\mathrm{C}-1), 100.5\left(\mathrm{C}-1^{\prime}\right), 109.2,127.6,127.75,127.78$, 127.83, 128.1, 128.2, 128.4, 128.5, 137.76, 137.80, 138.5; FAB-HRMS $\mathrm{m} / \mathrm{z}$ calcd for $\mathrm{C}_{37} \mathrm{H}_{46} \mathrm{~N}_{3} \mathrm{O}_{9}(\mathrm{M}+\mathrm{H})^{+} 676.3236$, found 676.3252; Anal. calcd for: $\mathrm{C}_{37} \mathrm{H}_{45} \mathrm{~N}_{3} \mathrm{O}_{9}$ : C, 65.76; $\mathrm{H}$, $6.71 ; \mathrm{N}, 6.22$, found $\mathrm{C}, 65.65 ; \mathrm{H}, 6.69 ; \mathrm{N}, 6.17$. Data for $\alpha-$ anomer $(\mathbf{2 8} \alpha)$ : TLC $R_{f}=0.63\left(2: 1\right.$ hexane/AcOEt); $[\alpha]_{\mathrm{D}}{ }^{23}$ $+80.8^{\circ}\left(c 0.99, \mathrm{CHCl}_{3}\right)$; IR (film) 2986, 2934, 2112, 1496, 1454, 1367, 1221, $1091 \mathrm{~cm}^{-1} ;{ }^{1} \mathrm{H}$ NMR (500 MHz, $\mathrm{CDCl}_{3}$ ) $\delta 1.26\left(\mathrm{~s}, 3 \mathrm{H}, \mathrm{CCH}_{3}\right), 1.34(\mathrm{~d}, J=6.3 \mathrm{~Hz}, 3 \mathrm{H}, \mathrm{H}-6), 1.37$ (s, $\left.3 \mathrm{H}, \mathrm{CCH}_{3}\right), 3.32(\mathrm{dd}, J=6.4,10.1 \mathrm{~Hz}, 1 \mathrm{H}, \mathrm{H}-4), 3.35$ (s, $3 \mathrm{H}, \mathrm{OCH}_{3}$ ), 3.50 (dd, $\left.J=4.6,8.4 \mathrm{~Hz}, 1 \mathrm{H}, \mathrm{H}-6{ }^{\prime} \mathrm{a}\right), 3.67$ (dd, $\left.J=8.4,9.5 \mathrm{~Hz}, 1 \mathrm{H}, \mathrm{H}-6^{\prime} \mathrm{b}\right), 3.69(\mathrm{dq}, J=10.1,6.3 \mathrm{~Hz}, 1 \mathrm{H}$, H-5), 3.89 (dd, $\left.J=3.4,10.7 \mathrm{~Hz}, 1 \mathrm{H}, \mathrm{H}-2^{\prime}\right), 3.95$ (dd, $J=$ 2.5, $\left.10.7 \mathrm{~Hz}, 1 \mathrm{H}, \mathrm{H}-3^{\prime}\right), 4.07-4.10$ (m, 2H, H-2, H-3), 4.16 (brs, $1 \mathrm{H}, \mathrm{H}-4^{\prime}$ ), 4.24 (dd, $\left.J=4.6,9.5 \mathrm{~Hz}, 1 \mathrm{H}, \mathrm{H}-5^{\prime}\right), 4.40$ (d, $J=11.9 \mathrm{~Hz}, 1 \mathrm{H}, \mathrm{OC} H \mathrm{Ph}), 4.50(\mathrm{~d}, J=11.9 \mathrm{~Hz}, 1 \mathrm{H}$, OCHPh), $4.57(\mathrm{~d}, J=11.2 \mathrm{~Hz}, 1 \mathrm{H}, \mathrm{OCHPh}), 4.64(\mathrm{~d}, J=$ $11.2 \mathrm{~Hz}, 1 \mathrm{H}, \mathrm{OCHPh}), 4.72(\mathrm{~d}, J=11.2 \mathrm{~Hz}, 1 \mathrm{H}, \mathrm{OCHPh})$, $4.83(\mathrm{~s}, 1 \mathrm{H}, \mathrm{H}-1), 4.89$ (d, $J=11.2 \mathrm{~Hz}, 1 \mathrm{H}, \mathrm{OCHPh}), 4.98$ 
$\left(\mathrm{d}, J=3.4 \mathrm{~Hz}, 1 \mathrm{H}, \mathrm{H}-1^{\prime}\right), 7.24-7.40(\mathrm{~m}, 15 \mathrm{H}, \mathrm{Ar}-\mathrm{H}) ;{ }^{13} \mathrm{C}$ NMR $\left(126 \mathrm{MHz}, \mathrm{CDCl}_{3}\right) \delta 17.5,26.4,27.9,54.8,60.1$, $64.8,67.5,69.0,71.9,73.1,73.5,74.9,76.0,76.9,80.4$, 97.9, 98.8, 109.1, 127.5, 127.8, 127.9, 128.0, 128.2, 128.45, 128.49, 137.6, 138.0, 138.6; FAB-HRMS $\mathrm{m} / \mathrm{z}$ calcd for $\mathrm{C}_{37} \mathrm{H}_{46} \mathrm{~N}_{3} \mathrm{O}_{9}(\mathrm{M}+\mathrm{H})^{+}$676.3234, found 676.3232.

\subsubsection{Methyl 2-azido-4- $O$-(2-azido-3,4,6-tri- $O$-benzyl-2- deoxy-D-galactopyranosyl)-3,6-di- $O$-benzyl-2-deoxy- $\beta$ -}

D-glucopyranoside (29). The glycosidation was performed according to the typical procedure $\left(1.5 \mathrm{~mL} \mathrm{EtCN},-78{ }^{\circ} \mathrm{C}\right.$, $0.5 \mathrm{~h})$ employing diphenyl phosphate $4 \mathrm{a}(70.8 \mathrm{mg}, 0.10$ $\mathrm{mmol}$ ), alcohol 24 (43.9 $\mathrm{mg}, 0.11 \mathrm{mmol}$ ), and TMSOTf (1.0 M in $\mathrm{CH}_{2} \mathrm{Cl}_{2}, 0.15 \mathrm{~mL}, 0.15 \mathrm{mmol}$ ). An anomeric mixture of disaccharide 29 (73.7 $\mathrm{mg}, 86 \%, \alpha: \beta=8: 92)$ was obtained as a colorless oil from the crude product (100.3 $\mathrm{mg}$ ) after column chromatography (silica gel $6 \mathrm{~g}, 30: 1$ toluene/AcOEt). The anomeric ratio of the product was determined by HPLC analysis [eluent, 5:1 hexane/AcOEt; flow rate, $1.0 \mathrm{~mL} / \mathrm{min} ; t_{\mathrm{R}}(\alpha$-anomer $)=10.2 \mathrm{~min}, t_{\mathrm{R}}(\beta$ anomer) $=14.8 \mathrm{~min}]$. The $\alpha$ - and $\beta$-glycosides were separated by flash column chromatography with 30:1 toluene/AcOEt. Data for $\beta$-anomer $(\mathbf{2 9} \beta)$ : TLC $R_{f}=0.53$ $\left(2: 1\right.$ hexane/AcOEt), $0.53(10: 1$ toluene/AcOEt $) ;[\alpha]_{\mathrm{D}}{ }^{22}$ $-39.7^{\circ}\left(c 0.93, \mathrm{CHCl}_{3}\right.$ ); IR (film) 3030, 2868, 2110, 1496, 1454, 1361, 1280, $1059 \mathrm{~cm}^{-1} ;{ }^{1} \mathrm{H}$ NMR $\left(500 \mathrm{MHz}, \mathrm{CDCl}_{3}\right)$ $\delta 3.15\left(\mathrm{dd}, J=2.8,10.4 \mathrm{~Hz}, 1 \mathrm{H}, \mathrm{H}-3^{\prime}\right), 3.23(\mathrm{dd}, J=5.1$, $\left.8.4 \mathrm{~Hz}, 1 \mathrm{H}, \mathrm{H}-5^{\prime}\right), 3.29$ (dd, $\left.J=5.1,9.0 \mathrm{~Hz}, 1 \mathrm{H}, \mathrm{H}-6^{\prime} \mathrm{a}\right)$, 3.35 (dd, $J=7.6,9.8 \mathrm{~Hz}, 1 \mathrm{H}, \mathrm{H}-2), 3.38$ (dd, $J=8.3,9.8$ $\mathrm{Hz}, 1 \mathrm{H}, \mathrm{H}-3$ ), 3.43 (ddd, $J=1.2,3.5,9.8 \mathrm{~Hz}, 1 \mathrm{H}, \mathrm{H}-5$ ), 3.48 (dd, $\left.J=8.4,9.0 \mathrm{~Hz}, 1 \mathrm{H}, \mathrm{H}-66^{\prime} \mathrm{b}\right), 3.55$ (s, $3 \mathrm{H}, \mathrm{OCH}_{3}$ ), $3.75\left(\mathrm{dd}, J=8.2,10.4 \mathrm{~Hz}, 1 \mathrm{H}, \mathrm{H}-2^{\prime}\right), 3.80(\mathrm{dd}, J=1.2,11.1$ $\mathrm{Hz}, 1 \mathrm{H}, \mathrm{H}-6 \mathrm{a}), 3.87$ (d, $\left.J=2.8 \mathrm{~Hz}, 1 \mathrm{H}, \mathrm{H}-4^{\prime}\right), 3.92$ (dd, $J=$ $3.5,11.1 \mathrm{~Hz}, 1 \mathrm{H}, \mathrm{H}-6 \mathrm{~b}), 4.01$ (dd, $J=8.3,9.8 \mathrm{~Hz}, 1 \mathrm{H}, \mathrm{H}-4)$, $4.12(\mathrm{~d}, J=7.6 \mathrm{~Hz}, 1 \mathrm{H}, \mathrm{H}-1), 4.23(\mathrm{~d}, J=11.8 \mathrm{~Hz}, 1 \mathrm{H}$, OCHPh), $4.25\left(\mathrm{~d}, J=8.2 \mathrm{~Hz}, 1 \mathrm{H}, \mathrm{H}^{-1} 1^{\prime}\right), 4.32(\mathrm{~d}, J=11.8$ $\mathrm{Hz}, 1 \mathrm{H}, \mathrm{OCHPh}), 4.47$ (d, $J=12.1 \mathrm{~Hz}, 1 \mathrm{H}, \mathrm{OCHPh}), 4.51$ $(\mathrm{d}, J=11.2 \mathrm{~Hz}, 1 \mathrm{H}, \mathrm{OCHPh}), 4.62(\mathrm{~d}, J=11.8 \mathrm{~Hz}, 1 \mathrm{H}$, $\mathrm{OCHPh}), 4.65(\mathrm{~d}, J=10.3 \mathrm{~Hz}, 1 \mathrm{H}, \mathrm{OCHPh}), 4.68(\mathrm{~d}, J=$ $11.8 \mathrm{~Hz}, 1 \mathrm{H}, \mathrm{OCHPh}), 4.69$ (d, $J=12.1 \mathrm{~Hz}, 1 \mathrm{H}, \mathrm{OCHPh})$, $4.89(\mathrm{~d}, J=11.2 \mathrm{~Hz}, 1 \mathrm{H}, \mathrm{OCHPh}), 4.98(\mathrm{~d}, J=10.3 \mathrm{~Hz}, 1 \mathrm{H}$, OCHPh), 7.12-7.38 (m, 25H, Ar-H); ${ }^{13} \mathrm{C}$ NMR (126 MHz, $\left.\mathrm{CDCl}_{3}\right) \delta 57.1,63.8,65.8,67.7,68.0,72.20,72.22,73.2$, 73.3, 73.4, 74.8, 74.9, 75.3, 76.1, 80.8, 81.4, $101.1\left(\mathrm{C}-1^{\prime}\right)$, 102.8 (C-1), 127.4, 127.5, 127.66, 127.74, 127.8, 127.9, $128.0,128.2,128.3,128.35,128.39,128.5,137.6,137.9$, 138.1, 138.2, 138.6; FAB-HRMS $\mathrm{m} / z$ calcd for $\mathrm{C}_{48} \mathrm{H}_{53} \mathrm{~N}_{6} \mathrm{O}_{9}$ $(\mathrm{M}+\mathrm{H})^{+}$857.3874, found 857.3879; Anal. calcd for: $\mathrm{C}_{48} \mathrm{H}_{52} \mathrm{~N}_{6} \mathrm{O}_{9}$ : C, 67.27; $\mathrm{H}, 6.12 ; \mathrm{N}, 9.81$, found $\mathrm{C}, 67.36 ; \mathrm{H}$, 6.15; N, 9.89. Data for $\alpha$-anomer $(29 \alpha)$ : TLC $R_{f}=0.59(2: 1$ hexane/AcOEt), 0.67 (10:1 toluene/AcOEt); $[\alpha]_{\mathrm{D}}^{22}+27.4^{\circ}$ (c 1.07, $\mathrm{CHCl}_{3}$ ); IR (film) 3030, 2868, 2110, 1496, 1454, 1361, 1280, $1059 \mathrm{~cm}^{-1}$; ${ }^{1} \mathrm{H}$ NMR $\left(500 \mathrm{MHz}, \mathrm{CDCl}_{3}\right) \delta$ 3.39-3.42 (m, 2H, H-2, H-6'a), 3.46 (dd, $J=6.4,9.0 \mathrm{~Hz}$, $\left.1 \mathrm{H}, \mathrm{H}-6^{\prime} \mathrm{b}\right), 3.49$ (m, 1H, H-5), 3.51 (dd, $J=9.1,9.5 \mathrm{~Hz}$, $1 \mathrm{H}, \mathrm{H}-3), 3.57$ (s, 3H, OCH $\left.H_{3}\right), 3.66(\mathrm{dd}, J=5.0,11.0 \mathrm{~Hz}$, 1H, H-6a), 3.74 (dd, $J=2.3,11.0 \mathrm{~Hz}, 1 \mathrm{H}, \mathrm{H}-6 \mathrm{~b}$ ), 3.76 (dd, $\left.J=2.3,10.9 \mathrm{~Hz}, 1 \mathrm{H}, \mathrm{H}-3^{\prime}\right), 3.81(\mathrm{dd}, J=3.7,10.9 \mathrm{~Hz}, 1 \mathrm{H}$, $\left.\mathrm{H}-2^{\prime}\right), 3.83(\mathrm{dd}, J=9.1,9.2 \mathrm{~Hz}, 1 \mathrm{H}, \mathrm{H}-4), 3.86(\mathrm{t}, J=6.4$ $\left.\mathrm{Hz}, 1 \mathrm{H}, \mathrm{H}-5^{\prime}\right), 3.95$ (brs, $\left.1 \mathrm{H}, \mathrm{H}-4^{\prime}\right), 4.22$ (d, $J=8.0 \mathrm{~Hz}, 1 \mathrm{H}$, $\mathrm{H}-1), 4.28$ (d, $J=11.7 \mathrm{~Hz}, 1 \mathrm{H}, \mathrm{OCHPh}), 4.36$ (d, $J=11.7$
$\mathrm{Hz}, 1 \mathrm{H}, \mathrm{OCHPh}), 4.46(\mathrm{~d}, J=12.4 \mathrm{~Hz}, 1 \mathrm{H}, \mathrm{OCHPh}), 4.49$ (d, $J=11.3 \mathrm{~Hz}, 1 \mathrm{H}, \mathrm{OC} H \mathrm{Ph}), 4.572(\mathrm{~d}, J=12.4 \mathrm{~Hz}, 1 \mathrm{H}$, $\mathrm{OCHPh}), 4.574(\mathrm{~d}, J=11.2 \mathrm{~Hz}, 1 \mathrm{H}, \mathrm{OCHPh}), 4.64(\mathrm{~d}, J=$ $11.2 \mathrm{~Hz}, 1 \mathrm{H}, \mathrm{OCHPh}), 4.80$ (d, $J=11.3 \mathrm{~Hz}, 1 \mathrm{H}, \mathrm{OCHPh})$, $4.84(\mathrm{~d}, J=10.4 \mathrm{~Hz}, 1 \mathrm{H}, \mathrm{OCHPh}), 5.00$ (d, $J=10.4 \mathrm{~Hz}, 1 \mathrm{H}$, OCHPh), $5.63\left(\mathrm{~d}, J=3.7 \mathrm{~Hz}, 1 \mathrm{H}, \mathrm{H}_{-1}{ }^{\prime}\right), 7.21-7.39(\mathrm{~m}, 25 \mathrm{H}$, Ar-H); ${ }^{13} \mathrm{C}$ NMR $\left(126 \mathrm{MHz}, \mathrm{CDCl}_{3}\right) \delta$ 57.0, 59.5, 66.7, 68.5, 69.6, 70.2, 72.0, 72.9, 73.3, 73.4, 73.5, 74.6, 74.76, 74.78, 83.7, $97.9\left(\mathrm{C}-1^{\prime}\right), 102.9$ (C-1), 127.5, 127.6, 127.7, $127.78,127.80,127.9,128.2,128.3,128.4,128.49$, 128.51, 137.5, 137.7, 137.8, 138.2, 138.3; FAB-HRMS $\mathrm{m} / \mathrm{z}$ calcd for $\mathrm{C}_{48} \mathrm{H}_{53} \mathrm{~N}_{6} \mathrm{O}_{9}(\mathrm{M}+\mathrm{H})^{+}$857.3874, found 857.3863.

\subsection{Glycosidations of 3,4,6-tri- $O$-acetyl-2-azido-2- deoxyglycosyl diphenyl phosphates $2 \mathrm{~b}$ and $4 \mathrm{~b}$}

4.4.1. Methyl 2,3,4-tri- $O$-benzyl-6- $O$-[1-(3,4,6-tri- $O$-acetyl-2-azido-2-deoxy-D-glucopyranosyl)iminopropyl]- $\alpha$ D-glucopyranoside (30). The glycosidation was performed according to the typical procedure $\left(1.5 \mathrm{~mL} \mathrm{EtCN},-65^{\circ} \mathrm{C}, 4\right.$ h) employing diphenyl phosphate $2 \mathbf{b}(56.3 \mathrm{mg}, 0.10 \mathrm{mmol})$, alcohol 7 (51.1 mg, 0.11 mmol), and TMSOTf (1.0 $\mathrm{M}$ in $\left.\mathrm{CH}_{2} \mathrm{Cl}_{2}, 0.15 \mathrm{~mL}, 0.15 \mathrm{mmol}\right)$. A mixture of imidate 30 and disaccharide $31(79.7 \mathrm{mg})$ was obtained as a colorless oil from the crude product (106.9 $\mathrm{mg})$ after short column chromatography (silica gel $3 \mathrm{~g}, 2: 1$ hexane/AcOEt with $1 \%$ $\mathrm{Et}_{3} \mathrm{~N}$ ). The anomeric ratio of the products was determined by HPLC analysis [eluent, 5:1:1 hexane/AcOEt/THF; flow rate, $1.0 \mathrm{~mL} / \mathrm{min} ; t_{\mathrm{R}}(\mathbf{3 0} \alpha)=9.6 \mathrm{~min}, t_{\mathrm{R}}(\mathbf{3 1} \alpha)=10.3 \mathrm{~min}$, $\left.t_{\mathrm{R}}(\mathbf{3 1} \beta)=11.1 \mathrm{~min}, t_{\mathrm{R}}(\mathbf{3 0} \beta)=11.8 \mathrm{~min}\right]$. The mixture was purified by flash column chromatography (silica gel $6 \mathrm{~g}$, $3: 1$ hexane/AcOEt with $1 \% \mathrm{Et}_{3} \mathrm{~N}$ ) to give $\alpha$-imidate $\mathbf{3 0} \alpha$ $(65.5 \mathrm{mg}, 79 \%)$ as a colorless oil, along with an anomeric mixture of disaccharide 31 (3.4 $\mathrm{mg}, 4 \%, \alpha: \beta=14: 86)$ as a colorless oil. The $\alpha$ - and $\beta$-glycosides of disaccharide 31 were separated by flash column chromatography with $15: 1$ toluene/acetone. Data for $\alpha$-anomer $(30 \alpha)$ : TLC $R_{f}=0.46$ $(1: 1$ hexane/AcOEt $) ;[\alpha]_{\mathrm{D}}^{23}+87.7^{\circ}\left(c\right.$ 2.01, $\left.\mathrm{CHCl}_{3}\right)$; IR (film) 2922, 2106, 1751, 1660, 1454, 1367, 1228, 1049 $\mathrm{cm}^{-1} ;{ }^{1} \mathrm{H}$ NMR $\left(500 \mathrm{MHz}, \mathrm{CDCl}_{3}\right) \delta 1.14(\mathrm{t}, J=7.6 \mathrm{~Hz}, 3 \mathrm{H}$, $\mathrm{CH}_{2} \mathrm{CH}_{3}$ ), 2.01 (s, 3H, $\mathrm{CH}_{3} \mathrm{CO}$ ), 2.06 (s, 3H, $\mathrm{CH}_{3} \mathrm{CO}$ ), 2.08 (s, 3H, $\left.\mathrm{CH}_{3} \mathrm{CO}\right), 2.35$ (q, J = 7.6 Hz, 2H, $\left.\mathrm{CH}_{2} \mathrm{CH}_{3}\right), 3.41$ (s, $\left.3 \mathrm{H}, \mathrm{OCH}_{3}\right), 3.52\left(\mathrm{dd}, J=4.1,10.2 \mathrm{~Hz}, 1 \mathrm{H}, \mathrm{H}-2^{\prime}\right), 3.55$ (dd, $J=3.5,9.6 \mathrm{~Hz}, 1 \mathrm{H}, \mathrm{H}-2), 3.61(\mathrm{dd}, J=8.9,10.1 \mathrm{~Hz}, 1 \mathrm{H}$, $\mathrm{H}-4), 3.90$ (ddd, $J=1.5,4.0,10.1 \mathrm{~Hz}, 1 \mathrm{H}, \mathrm{H}-5), 3.95(\mathrm{~m}$, $1 \mathrm{H}, \mathrm{H}-6 \mathrm{a}), 4.01$ (dd, $J=8.9,9.6 \mathrm{~Hz}, 1 \mathrm{H}, \mathrm{H}-3), 4.22-4.26$ (m, 3H, H-6b, H-5', H-6'a), 4.42 (dd, $J=1.7,12.3 \mathrm{~Hz}, 1 \mathrm{H}$, H-6'b), 4.60 (d, J = 3.5 Hz, 1H, H-1), 4.61 (d, $J=10.7 \mathrm{~Hz}$, $1 \mathrm{H}, \mathrm{OCHPh}), 4.67(\mathrm{~d}, J=12.0 \mathrm{~Hz}, 1 \mathrm{H}, \mathrm{OCHPh}), 4.81(\mathrm{~d}, J$ $=12.0 \mathrm{~Hz}, 1 \mathrm{H}, \mathrm{OCHPh}), 4.84(\mathrm{~d}, J=10.8 \mathrm{~Hz}, 1 \mathrm{H}, \mathrm{OCHPh})$, $4.85(\mathrm{~d}, J=10.7 \mathrm{~Hz}, 1 \mathrm{H}, \mathrm{OCHPh}), 4.99(\mathrm{~d}, J=10.8 \mathrm{~Hz}, 1 \mathrm{H}$, OCHPh), $5.08\left(\mathrm{t}, J=9.6 \mathrm{~Hz}, 1 \mathrm{H}, \mathrm{H}-4^{\prime}\right), 5.22(\mathrm{~d}, J=4.1 \mathrm{~Hz}$, $\left.1 \mathrm{H}, \mathrm{H}-1^{\prime}\right), 5.63\left(\mathrm{dd}, J=9.6,10.2 \mathrm{~Hz}, 1 \mathrm{H}, \mathrm{H}-3^{\prime}\right), 7.26-7.38$ (m, 15H, Ar-H); ${ }^{13} \mathrm{C}$ NMR (126 MHz, $\left.\mathrm{CDCl}_{3}\right) \delta 10.6,20.6$, 20.69, 20.73, 23.0, 55.2, 62.1, 62.2, 64.6, 68.0, 68.7, 69.2, 71.4, 73.4, 75.1, 75.8, 77.6, 80.0, 82.0, 83.0 (C-1'), 98.1 (C1), 127.6, 127.8, 127.9, 128.0, 128.06, 128.11, 128.39, $128.44,137.9,138.1,138.6,169.7,169.9,170.3,170.6$; FAB-HRMS $\mathrm{m} / z$, calcd for $\mathrm{C}_{43} \mathrm{H}_{53} \mathrm{~N}_{4} \mathrm{O}_{13}(\mathrm{M}+\mathrm{H})^{+} 833.3609$, found 833.3600; Anal. calcd for: $\mathrm{C}_{43} \mathrm{H}_{52} \mathrm{~N}_{4} \mathrm{O}_{13}$ : C, 62.01; $\mathrm{H}$, $6.30 ; \mathrm{N}, 6.73$, found $\mathrm{C}, 61.85 ; \mathrm{H}, 6.23 ; \mathrm{N}, 6.64$. Data for $\beta$ - 
anomer $(\mathbf{3 0} \beta)$ : TLC $R_{f}=0.40(1: 1$ hexane/AcOEt $) ;[\alpha]_{\mathrm{D}}{ }^{21}$ $+9.07^{\circ}\left(c 0.45, \mathrm{CHCl}_{3}\right)$; IR (film) 2924, 2112, 1751, 1657, 1454, 1365, 1230, $1047 \mathrm{~cm}^{-1} ;{ }^{1} \mathrm{H}$ NMR $\left(500 \mathrm{MHz}, \mathrm{CDCl}_{3}\right)$ $\delta 1.16\left(\mathrm{t}, J=7.4 \mathrm{~Hz}, 3 \mathrm{H}, \mathrm{CH}_{2} \mathrm{CH}_{3}\right), 2.01$ (s, 3H, $\left.\mathrm{CH}_{3} \mathrm{CO}\right)$, $2.02\left(\mathrm{~s}, 3 \mathrm{H}, \mathrm{CH}_{3} \mathrm{CO}\right), 2.09\left(\mathrm{~s}, 3 \mathrm{H}, \mathrm{CH}_{3} \mathrm{CO}\right), 2.34(\mathrm{dq}, J=$ 14.6, 7.4 Hz, 1H, $\left.\mathrm{CHCH}_{3}\right), 2.36(\mathrm{dq}, J=14.6,7.4 \mathrm{~Hz}, 1 \mathrm{H}$, $\left.\mathrm{CHCH}_{3}\right), 3.37\left(\mathrm{~s}, 3 \mathrm{H}, \mathrm{OCH}_{3}\right), 3.515(\mathrm{dd}, J=8.4,9.4 \mathrm{~Hz}$, $\left.1 \mathrm{H}, \mathrm{H}-2^{\prime}\right), 3.523$ (dd, $\left.J=9.0,10.1 \mathrm{~Hz}, 1 \mathrm{H}, \mathrm{H}-4\right), 3.54$ (dd, $J$ $=3.6,9.5 \mathrm{~Hz}, 1 \mathrm{H}, \mathrm{H}-2), 3.75(\mathrm{ddd}, J=2.1,5.6,9.7 \mathrm{~Hz}, 1 \mathrm{H}$, H-5'), 3.87 (ddd, $J=1.8,4.7,10.1 \mathrm{~Hz}, 1 \mathrm{H}, \mathrm{H}-5), 4.01$ (dd, $J=9.0,9.5 \mathrm{~Hz}, 1 \mathrm{H}, \mathrm{H}-3), 4.09(\mathrm{dd}, J=2.1,12.3 \mathrm{~Hz}, 1 \mathrm{H}$, H-6'a), 4.18 (dd, $J=5.6,12.3 \mathrm{~Hz}, 1 \mathrm{H}, \mathrm{H}-6^{\prime} \mathrm{b}$ ), 4.30 (dd, $J=$ $1.8,12.2 \mathrm{~Hz}, 1 \mathrm{H}, \mathrm{H}-6 \mathrm{a}), 4.42$ (dd, $J=4.7,12.2 \mathrm{~Hz}, 1 \mathrm{H}, \mathrm{H}-$ $6 \mathrm{~b}), 4.51$ (d, $\left.J=8.4 \mathrm{~Hz}, 1 \mathrm{H}, \mathrm{H}-1^{\prime}\right), 4.56(\mathrm{~d}, J=10.7 \mathrm{~Hz}, 1 \mathrm{H}$, OCHPh), 4.61 (d, $J=3.6 \mathrm{~Hz}, 1 \mathrm{H}, \mathrm{H}-1), 4.67(\mathrm{~d}, J=12.1$ $\mathrm{Hz}, 1 \mathrm{H}, \mathrm{OC} H \mathrm{Ph}), 4.80$ (d, $J=12.1 \mathrm{~Hz}, 1 \mathrm{H}, \mathrm{OC} H \mathrm{Ph}), 4.82$ $(\mathrm{d}, J=10.8 \mathrm{~Hz}, 1 \mathrm{H}, \mathrm{OCHPh}), 4.87(\mathrm{~d}, J=10.7 \mathrm{~Hz}, 1 \mathrm{H}$, OCHPh), $4.99(\mathrm{~d}, J=10.8 \mathrm{~Hz}, 1 \mathrm{H}, \mathrm{OC} H \mathrm{Ph}), 5.00(\mathrm{dd}, J=$ 8.2, $9.7 \mathrm{~Hz}, 1 \mathrm{H}, \mathrm{H}-4^{\prime}$ ), 5.04 (dd, $J=8.2,9.4 \mathrm{~Hz}, 1 \mathrm{H}, \mathrm{H}-3^{\prime}$ ), 7.26-7.37 (m, 15H, Ar-H); ${ }^{13} \mathrm{C}$ NMR (126 MHz, CDCl $\left.{ }_{3}\right) \delta$ 10.8, 20.6, 20.68, 20.74, 23.9, 55.1, 62.6, 64.6, 65.8, 68.8, 73.0, 73.4, 73.7, 75.1, 75.9, 78.0, 80.0, 82.1, 87.8 (C-1'), 98.0 (C-1), 127.7, 127.8, 127.88, 127.93, 128.07, 128.09, $128.41,128.43,128.5,138.1,138.2$, 138.7, 169.7, 170.1, 170.6, 171.9; FAB-HRMS $m / z$ calcd for $\mathrm{C}_{43} \mathrm{H}_{53} \mathrm{~N}_{4} \mathrm{O}_{13}$ $(\mathrm{M}+\mathrm{H})^{+} 833.3609$, found 833.3580.

Data for methyl 2,3,4-tri- $O$-benzyl-6- $O$-(3,4,6-tri- $O$-acetyl2 -azido-2-deoxy-D-glucopyranosyl)- $\alpha$-D-glucopyranoside (31). Data for $\beta$-anomer (31 $\beta)$ : TLC $R_{f}=0.40$ (1:1 hexane/ AcOEt), 0.52 (5:1 toluene/acetone); $[\alpha]_{\mathrm{D}}{ }^{17}-1.41^{\circ}$ (c 1.58, $\mathrm{CHCl}_{3}$ ); IR (film) 2930, 2112, 1753, 1454, 1365, 1228, $1049 \mathrm{~cm}^{-1}$; ${ }^{1} \mathrm{H}$ NMR $\left(500 \mathrm{MHz}, \mathrm{CDCl}_{3}\right) \delta 2.01(\mathrm{~s}, 3 \mathrm{H}$, $\mathrm{CH}_{3} \mathrm{CO}$ ), 2.04 (s, 3H, CH $\mathrm{CO}$ ), 2.08 (s, 3H, CH $\mathrm{CH}_{3} \mathrm{CO}$ ), 3.38 $\left(\mathrm{s}, 3 \mathrm{H}, \mathrm{OCH}_{3}\right), 3.52$ (dd, $\left.J=9.2,10.1 \mathrm{~Hz}, 1 \mathrm{H}, \mathrm{H}-4\right)$, $3.52-3.55\left(\mathrm{~m}, 2 \mathrm{H}, \mathrm{H}-2, \mathrm{H}-2^{\prime}\right), 3.57$ (ddd, $J=2.4,3.6,9.5$ $\left.\mathrm{Hz}, 1 \mathrm{H}, \mathrm{H}-5^{\prime}\right), 3.70$ (dd, $\left.J=4.7,10.9 \mathrm{~Hz}, 1 \mathrm{H}, \mathrm{H}-6 \mathrm{a}\right), 3.82$ (ddd, $J=1.7,4.7,10.1 \mathrm{~Hz}, 1 \mathrm{H}, \mathrm{H}-5), 4.00$ (dd, $J=9.2,9.3$ $\mathrm{Hz}, 1 \mathrm{H}, \mathrm{H}-3), 4.090$ (dd, $J=1.7,10.9 \mathrm{~Hz}, 1 \mathrm{H}, \mathrm{H}-6 \mathrm{~b}), 4.094$ $\left(\mathrm{dd}, J=2.4,12.1 \mathrm{~Hz}, 1 \mathrm{H}, \mathrm{H}-6{ }^{\prime} \mathrm{a}\right), 4.22(\mathrm{dd}, J=3.6,12.1 \mathrm{~Hz}$, $\left.1 \mathrm{H}, \mathrm{H}-6^{\prime} \mathrm{b}\right), 4.23$ (d, $\left.J=8.1 \mathrm{~Hz}, 1 \mathrm{H}, \mathrm{H}-1^{\prime}\right), 4.61$ (d, $J=3.5$ $\mathrm{Hz}, 1 \mathrm{H}, \mathrm{H}-1), 4.62(\mathrm{~d}, J=11.0 \mathrm{~Hz}, 1 \mathrm{H}, \mathrm{OCHPh}), 4.65(\mathrm{~d}, J$ $=12.1 \mathrm{~Hz}, 1 \mathrm{H}, \mathrm{OCHPh}), 4.79(\mathrm{~d}, J=12.1 \mathrm{~Hz}, 1 \mathrm{H}, \mathrm{OCHPh})$, $4.82(\mathrm{~d}, J=11.0 \mathrm{~Hz}, 1 \mathrm{H}, \mathrm{OCHPh}), 4.94(\mathrm{~d}, J=11.0 \mathrm{~Hz}, 1 \mathrm{H}$, $\mathrm{OCHPh}), \quad 4.96-5.00 \quad\left(\mathrm{~m}, \quad 3 \mathrm{H}, \quad \mathrm{H}-3^{\prime}, \quad \mathrm{H}-4^{\prime}, \quad \mathrm{OCHPh}\right)$, 7.26-7.37 (m, 15H, Ar-H); ${ }^{13} \mathrm{C}$ NMR (126 MHz, $\left.\mathrm{CDCl}_{3}\right) \delta$ 20.5, 20.61, 20.63, 55.3, 61.8, 63.8, 68.4, 68.7, 69.7, 71.7, 72.6, 73.4, 74.8, 75.7, 77.6, 79.8, 82.0, 98.2 (C-1), 102.0 $\left(\mathrm{C}-1^{\prime}\right), 127.6,127.7,127.90,127.93,128.1,128.2,128.35$, $128.43,128.5,138.1,138.3,138.7,169.5,169.9,170.5$; FAB-HRMS $m / z$ calcd for $\mathrm{C}_{40} \mathrm{H}_{47} \mathrm{~N}_{3} \mathrm{O}_{13} \mathrm{Na} \quad(\mathrm{M}+\mathrm{Na})^{+}$ 800.3007, found 800.3033; Anal. calcd for: $\mathrm{C}_{40} \mathrm{H}_{47} \mathrm{~N}_{3} \mathrm{O}_{13}$ : C, 61.77; H, 6.09; N, 5.40, found C, 61.64; H, 6.08; N, 5.31. Data for $\alpha$-anomer $(31 \alpha)$ : TLC $R_{f}=0.42$ (1:1 hexane/ AcOEt), 0.56 (5:1 toluene/acetone); $[\alpha]_{\mathrm{D}}{ }^{20}+119.6^{\circ}$ (c 1.28, $\mathrm{CHCl}_{3}$ ); IR (film) 3030, 2932, 2108, 1751, 1454, 1367, 1226, $1047 \mathrm{~cm}^{-1}$; ${ }^{1} \mathrm{H}$ NMR $\left(500 \mathrm{MHz}, \mathrm{CDCl}_{3}\right) \delta 2.02(\mathrm{~s}, 3 \mathrm{H}$, $\mathrm{CH}_{3} \mathrm{CO}$ ), 2.03 (s, 3H, CH $\mathrm{CH}_{3} \mathrm{CO}, 2.08\left(\mathrm{~s}, 3 \mathrm{H}, \mathrm{CH}_{3} \mathrm{CO}\right), 3.30$ (dd, $\left.J=3.4,10.7 \mathrm{~Hz}, 1 \mathrm{H}, \mathrm{H}-2^{\prime}\right), 3.39$ (s, 3H, OCH$\left.H_{3}\right), 3.53$ (dd, $J=3.5,9.8 \mathrm{~Hz}, 1 \mathrm{H}, \mathrm{H}-2), 3.54$ (dd, $J=8.7,10.2 \mathrm{~Hz}$, 1H, H-4), 3.68 (m, 1H, H-6a), 3.77-3.81 (m, 2H, H-5, H- 6b), 3.92 (ddd, $\left.J=2.2,4.3,10.1 \mathrm{~Hz}, 1 \mathrm{H}, \mathrm{H}-5^{\prime}\right), 3.99$ (dd, $J$ $=2.2,12.5 \mathrm{~Hz}, 1 \mathrm{H}, \mathrm{H}-6 \mathrm{a}$ ), $4.01(\mathrm{dd}, J=8.7,9.8 \mathrm{~Hz}, 1 \mathrm{H}$, H-3), 4.15 (dd, $J=4.3,12.5 \mathrm{~Hz}, 1 \mathrm{H}, \mathrm{H}-6$ 'b), 4.59 (d, $J=$ $3.5 \mathrm{~Hz}, 1 \mathrm{H}, \mathrm{H}-1), 4.62$ (d, $J=11.5 \mathrm{~Hz}, 1 \mathrm{H}, \mathrm{OCHPh}), 4.66$ $(\mathrm{d}, J=12.0 \mathrm{~Hz}, 1 \mathrm{H}, \mathrm{OCHPh}), 4.78(\mathrm{~d}, J=12.0 \mathrm{~Hz}, 1 \mathrm{H}$, OCHPh), $4.81(\mathrm{~d}, J=11.2 \mathrm{~Hz}, 1 \mathrm{H}, \mathrm{OCHPh}), 4.97(\mathrm{~d}, J=$ $11.5 \mathrm{~Hz}, 1 \mathrm{H}, \mathrm{OCHPh}), 4.99$ (d, $J=11.2 \mathrm{~Hz}, 1 \mathrm{H}, \mathrm{OCHPh})$, $5.00\left(\mathrm{dd}, J=9.4,10.1 \mathrm{~Hz}, 1 \mathrm{H}, \mathrm{H}-4^{\prime}\right), 5.04(\mathrm{~d}, J=3.4 \mathrm{~Hz}$, $\left.1 \mathrm{H}, \mathrm{H}-1^{\prime}\right), 5.40\left(\mathrm{dd}, J=9.4,10.7 \mathrm{~Hz}, 1 \mathrm{H}, \mathrm{H}-3^{\prime}\right), 7.26-7.37$ $(\mathrm{m}, 15 \mathrm{H}, \mathrm{Ar}-\mathrm{H}) ;{ }^{13} \mathrm{C} \mathrm{NMR}\left(126 \mathrm{MHz}, \mathrm{CDCl}_{3}\right) \delta 20.57$, 20.64, 20.7, 55.2, 61.0, 61.7, 66.7, 67.5, 68.5, 69.9, 70.3, 73.4, 74.9, 75.7, 77.5, 80.0, 82.0, $97.95\left(\mathrm{C}-1^{\prime}\right), 98.04$ (C-1), $127.6,127.7,127.8,127.9,128.0,128.1,128.37,128.42$, 138.1, 138.3, 138.7, 169.6, 169.9, 170.5; FAB-HRMS m/z calcd for $\mathrm{C}_{40} \mathrm{H}_{47} \mathrm{~N}_{3} \mathrm{O}_{13} \mathrm{Na} \quad(\mathrm{M}+\mathrm{Na})^{+}$800.3007, found 800.3034 .

4.4.2. Methyl 2,3,4-tri- $O$-benzyl-6-O-[1-(3,4,6-tri- $O$-acetyl-2-azido-2-deoxy-D-galactopyranosyl)iminopropyl]- $\alpha$ D-glucopyranoside (32). The glycosidation was performed according to the typical procedure $\left(1.5 \mathrm{~mL} \mathrm{EtCN},-65^{\circ} \mathrm{C}, 3\right.$ h) employing diphenyl phosphate $4 \mathbf{b}(56.3 \mathrm{mg}, 0.10 \mathrm{mmol})$, alcohol 7 (51.1 mg, 0.11 mmol), and TMSOTf (1.0 $\mathrm{M}$ in $\left.\mathrm{CH}_{2} \mathrm{Cl}_{2}, 0.15 \mathrm{~mL}, 0.15 \mathrm{mmol}\right)$. A mixture of imidate 32 and disaccharide $\mathbf{3 3}(80.3 \mathrm{mg})$ was obtained as a colorless oil from the crude product $(102.4 \mathrm{mg})$ after short column chromatography (silica gel $3 \mathrm{~g}, 2: 1$ hexane/AcOEt with $1 \%$ $\mathrm{Et}_{3} \mathrm{~N}$ ). The anomeric ratio of the products was determined by HPLC analysis [eluent, 4:1 hexane/THF; flow rate, 1.0 $\mathrm{mL} / \mathrm{min} ; t_{\mathrm{R}}(\mathbf{3 2} \alpha)=19.3 \min , t_{\mathrm{R}}(\mathbf{3 3} \alpha)=21.7 \mathrm{~min}, t_{\mathrm{R}}(\mathbf{3 3} \beta)$ $\left.=26.6 \mathrm{~min}, t_{\mathrm{R}}(32 \beta)=30.4 \mathrm{~min}\right]$. The mixture was purified by flash column chromatography (silica gel $6 \mathrm{~g}, 4: 1$ hexane/acetone with $\left.1 \% \mathrm{Et}_{3} \mathrm{~N}\right)$ to give $\alpha$-imidate $32 \alpha(56.4$ $\mathrm{mg}, 68 \%)$ as a colorless oil, along with an anomeric mixture of disaccharide $33(6.8 \mathrm{mg}, 9 \%, \alpha: \beta=3: 97)$ as a white solid. The $\alpha$ - and $\beta$-glycosides of disaccharide 33 were separated by flash column chromatography with $1: 1$ hexane/Et ${ }_{2} \mathrm{O}$. Data for $\alpha$-anomer $(32 \alpha)$ : TLC $R_{f}=0.47(1: 1$ hexane/AcOEt); $[\alpha]_{\mathrm{D}}^{22}+72.7^{\circ}\left(c 1.50, \mathrm{CHCl}_{3}\right)$; IR (film) 2916, 2108, 1751, 1662, 1454, 1371, 1228, $1078 \mathrm{~cm}^{-1} ;{ }^{1} \mathrm{H}$ NMR $\left(500 \mathrm{MHz}, \mathrm{CDCl}_{3}\right) \delta 1.15(\mathrm{t}, J=7.6 \mathrm{~Hz}, 3 \mathrm{H}$, $\left.\mathrm{CH}_{2} \mathrm{CH}_{3}\right), 1.99$ (s, 3H, $\left.\mathrm{CH}_{3} \mathrm{CO}\right), 2.05$ (s, 3H, $\left.\mathrm{CH}_{3} \mathrm{CO}\right), 2.15$ (s, $\left.3 \mathrm{H}, \mathrm{CH}_{3} \mathrm{CO}\right), 2.35$ (q, J = 7.6 Hz, $2 \mathrm{H}, \mathrm{CH}_{2} \mathrm{CH}_{3}$ ), 3.40 (s, $\left.3 \mathrm{H}, \mathrm{OCH}_{3}\right), 3.55(\mathrm{dd}, J=3.6,9.6 \mathrm{~Hz}, 1 \mathrm{H}, \mathrm{H}-2), 3.60(\mathrm{dd}, J$ $=9.0,10.0 \mathrm{~Hz}, 1 \mathrm{H}, \mathrm{H}-4), 3.81(\mathrm{dd}, J=4.0,10.8 \mathrm{~Hz}, 1 \mathrm{H}, \mathrm{H}-$ $2^{\prime}$ ), 3.88 (ddd, $\left.J=1.6,4.3,10.0 \mathrm{~Hz}, 1 \mathrm{H}, \mathrm{H}-5\right), 3.97$ (dd, $J=$ $\left.6.8,11.3 \mathrm{~Hz}, 1 \mathrm{H}, \mathrm{H}-6^{\prime} \mathrm{a}\right), 4.01$ (dd, $J=9.0,9.6 \mathrm{~Hz}, 1 \mathrm{H}, \mathrm{H}-$ 3), 4.04 (dd, $\left.J=6.6,11.3 \mathrm{~Hz}, 1 \mathrm{H}, \mathrm{H}-6^{\prime} \mathrm{b}\right), 4.23$ (dd, $J=4.3$, $12.1 \mathrm{~Hz}, 1 \mathrm{H}, \mathrm{H}-6 \mathrm{a}), 4.36$ (dd, $J=1.6,12.1 \mathrm{~Hz}, 1 \mathrm{H}, \mathrm{H}-6 \mathrm{~b})$, 4.39 (ddd, $\left.J=0.7,6.6,6.8 \mathrm{~Hz}, 1 \mathrm{H}, \mathrm{H}-5^{\prime}\right), 4.59$ (d, $J=10.7$ $\mathrm{Hz}, 1 \mathrm{H}, \mathrm{OCHPh}), 4.61$ (d, $J=3.6 \mathrm{~Hz}, 1 \mathrm{H}, \mathrm{H}-1), 4.68(\mathrm{~d}, J$ $=12.1 \mathrm{~Hz}, 1 \mathrm{H}, \mathrm{OC} H \mathrm{Ph}), 4.81(\mathrm{~d}, J=12.1 \mathrm{~Hz}, 1 \mathrm{H}, \mathrm{OC} H \mathrm{Ph})$, $4.83(\mathrm{~d}, J=10.7 \mathrm{~Hz}, 1 \mathrm{H}, \mathrm{OCH \textrm {Ph }}), 4.85$ (d, $J=10.7 \mathrm{~Hz}, 1 \mathrm{H}$, OCHPh), $4.99(\mathrm{~d}, J=10.7 \mathrm{~Hz}, 1 \mathrm{H}, \mathrm{OCHPh}), 5.24(\mathrm{~d}, J=$ $4.0 \mathrm{~Hz}, 1 \mathrm{H}, \mathrm{H}-1^{\prime}$ ), 5.44 (dd, $J=0.7,3.3 \mathrm{~Hz}, 1 \mathrm{H}, \mathrm{H}-4^{\prime}$ ), 5.47 (dd, $\left.J=3.3,10.8 \mathrm{~Hz}, 1 \mathrm{H}, \mathrm{H}-3^{\prime}\right), 7.26-7.38$ (m, 15H, Ar-H); ${ }^{13} \mathrm{C}$ NMR $\left(126 \mathrm{MHz}, \mathrm{CDCl}_{3}\right) \delta 10.6,20.6,20.66,20.70$, 23.0, 55.2, 58.6, 62.0, 64.5, 67.0, 68.0, 68.7, 69.3, 73.4, 75.2, 75.8, 77.7, 80.0, 82.0, $83.4\left(\mathrm{C}^{\prime} \mathbf{1}^{\prime}\right), 98.1$ (C-1), 127.6, $127.8,127.9,128.00,128.02,128.1,128.4,128.5,138.0$, 138.1, 138.7, 169.8, 170.0, 170.1, 170.3; FAB-HRMS m/z 
calcd for $\mathrm{C}_{43} \mathrm{H}_{53} \mathrm{~N}_{4} \mathrm{O}_{13}(\mathrm{M}+\mathrm{H})^{+}$833.3609, found 833.3626; Anal. calcd for: $\mathrm{C}_{43} \mathrm{H}_{52} \mathrm{~N}_{4} \mathrm{O}_{13}$ : C, 62.01; H, 6.30; N, 6.73, found $\mathrm{C}, 61.95, \mathrm{H}, 6.14, \mathrm{~N}, 6.69$. Data for $\beta$-anomer $(32 \beta)$ : TLC $R_{f}=0.45\left(1: 1\right.$ hexane/AcOEt); ${ }^{1} \mathrm{H}$ NMR $(500 \mathrm{MHz}$, $\left.\mathrm{CDCl}_{3}\right) \delta 1.17\left(\mathrm{dd}, J=7.0,7.3 \mathrm{~Hz}, 3 \mathrm{H}, \mathrm{CH}_{2} \mathrm{CH}_{3}\right), 2.01(\mathrm{~s}$, $3 \mathrm{H}, \mathrm{CH}_{3} \mathrm{CO}$ ), 2.06 (s, 3H, $\left.\mathrm{CH}_{3} \mathrm{CO}\right), 2.15$ (s, 3H, $\mathrm{CH}_{3} \mathrm{CO}$ ), $2.35\left(\mathrm{dq}, J=14.4,7.3 \mathrm{~Hz}, 1 \mathrm{H}, \mathrm{CHCH}_{3}\right), 2.38(\mathrm{dq}, J=14.4$, $\left.7.0 \mathrm{~Hz}, 1 \mathrm{H}, \mathrm{CHCH}_{3}\right), 3.38\left(\mathrm{~s}, 3 \mathrm{H}, \mathrm{OCH}_{3}\right), 3.545(\mathrm{dd}, J=$ $3.6,9.7 \mathrm{~Hz}, 1 \mathrm{H}, \mathrm{H}-2), 3.546$ (dd, $J=8.9,9.9 \mathrm{~Hz}, 1 \mathrm{H}, \mathrm{H}-4)$, $3.80\left(\mathrm{dd}, J=8.3,10.9 \mathrm{~Hz}, 1 \mathrm{H}, \mathrm{H}-2^{\prime}\right), 3.88(\mathrm{ddd}, J=2.1,4.5$, $9.9 \mathrm{~Hz}, 1 \mathrm{H}, \mathrm{H}-5), 3.94\left(\mathrm{dd}, J=6.5,6.7 \mathrm{~Hz}, 1 \mathrm{H}, \mathrm{H}-5^{\prime}\right), 4.01$ $(\mathrm{dd}, J=8.9,9.7 \mathrm{~Hz}, 1 \mathrm{H}, \mathrm{H}-3), 4.10(\mathrm{dd}, J=6.5,11.4 \mathrm{~Hz}$, 1H, H-6'a), 4.12 (dd, $J=6.7,11.4 \mathrm{~Hz}, 1 \mathrm{H}, \mathrm{H}-6^{\prime} \mathrm{b}$ ), 4.35 (dd, $J=2.1,12.1 \mathrm{~Hz}, 1 \mathrm{H}, \mathrm{H}-6 \mathrm{a}), 4.39$ (dd, $J=4.5,12.1 \mathrm{~Hz}, 1 \mathrm{H}$, H-6b), 4.50 (d, $\left.J=8.3 \mathrm{~Hz}, 1 \mathrm{H}, \mathrm{H}-1^{\prime}\right), 4.57$ (d, $J=10.7 \mathrm{~Hz}$, $1 \mathrm{H}, \mathrm{OCHPh}), 4.61(\mathrm{~d}, J=3.6 \mathrm{~Hz}, 1 \mathrm{H}, \mathrm{H}-1), 4.67$ (d, $J=$ $12.1 \mathrm{~Hz}, 1 \mathrm{H}, \mathrm{OCHPh}), 4.80$ (d, $J=12.1 \mathrm{~Hz}, 1 \mathrm{H}, \mathrm{OCHPh})$, $4.82(\mathrm{~d}, J=10.7 \mathrm{~Hz}, 1 \mathrm{H}, \mathrm{OCHPh}), 4.85(\mathrm{dd}, J=3.4,10.9$ $\left.\mathrm{Hz}, 1 \mathrm{H}, \mathrm{H}-3^{\prime}\right), 4.86$ (d, J = $\left.10.7 \mathrm{~Hz}, 1 \mathrm{H}, \mathrm{OCHPh}\right), 4.98$ (d, $J=10.7 \mathrm{~Hz}, 1 \mathrm{H}, \mathrm{OC} H \mathrm{Ph}), 5.38\left(\mathrm{~d}, J=3.4 \mathrm{~Hz}, 1 \mathrm{H}, \mathrm{H}-4^{\prime}\right)$, 7.25-7.37 (m, 15H, Ar-H); ${ }^{13} \mathrm{C}$ NMR $\left(126 \mathrm{MHz}, \mathrm{CDCl}_{3}\right) \delta$ 10.8, 20.6, 20.66, 20.68, 23.8, 55.1, 61.8, 62.8, 64.7, 66.8, $68.7,71.3,72.5,73.4,75.2,75.9,78.1,80.1,82.1,88.3$ (C$\left.1^{\prime}\right)$, 98.0 (C-1), 127.67, 127.69, 127.9, 128.06, 128.08, $128.38,128.42,128.5,138.1,138.2,138.7,169.9,170.2$, $170.4,171.8$; FAB-HRMS $\mathrm{m} / \mathrm{z}$ calcd for $\mathrm{C}_{43} \mathrm{H}_{53} \mathrm{~N}_{4} \mathrm{O}_{13}$ $(\mathrm{M}+\mathrm{H})^{+}$833.3609, found 833.3614.

Data for methyl 2,3,4-tri- $O$-benzyl-6- $O$-(3,4,6-tri- $O$-acetyl2-azido-2-deoxy-D-galactopyranosyl)- $\alpha$-D-glucopyranoside (33). ${ }^{9}$ Data for $\beta$-anomer $(33 \beta):{ }^{9}$ TLC $R_{f}=0.42(1: 1$ hexane/AcOEt $), \quad 0.24 \quad\left(1: 3\right.$ hexane/Et $\left.{ }_{2} \mathrm{O}\right) ; \quad$ mp 145.0$146.0{ }^{\circ} \mathrm{C}$ (colorless needles from AcOEt-hexane); $[\alpha]_{\mathrm{D}}{ }^{24}$ $-9.46^{\circ}$ (c 1.00, $\mathrm{CHCl}_{3}$ ); IR (KBr) 3032, 2926, 2114, 1751, 1454, 1369, 1242, $1076 \mathrm{~cm}^{-1} ;{ }^{1} \mathrm{H}$ NMR $\left(500 \mathrm{MHz}, \mathrm{CDCl}_{3}\right)$

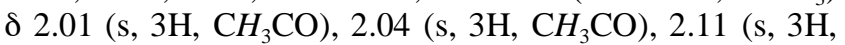
$\left.\mathrm{CH}_{3} \mathrm{CO}\right), 3.39\left(\mathrm{~s}, 3 \mathrm{H}, \mathrm{OCH}_{3}\right), 3.53(\mathrm{dd}, J=8.8,10.2 \mathrm{~Hz}$, $1 \mathrm{H}, \mathrm{H}-4), 3.54(\mathrm{dd}, J=3.5,9.6 \mathrm{~Hz}, 1 \mathrm{H}, \mathrm{H}-2), 3.70(\mathrm{dd}, J=$ $4.8,11.0 \mathrm{~Hz}, 1 \mathrm{H}, \mathrm{H}-6 \mathrm{a}), 3.72$ (dd, $J=8.1,10.9 \mathrm{~Hz}, 1 \mathrm{H}, \mathrm{H}-$ $\left.2^{\prime}\right), 3.77\left(\mathrm{dd}, J=6.8,7.0 \mathrm{~Hz}, 1 \mathrm{H}, \mathrm{H}-5^{\prime}\right), 3.83(\mathrm{ddd}, J=1.5$, $4.5,10.2 \mathrm{~Hz}, 1 \mathrm{H}, \mathrm{H}-5), 4.01$ (dd, $J=8.8,9.6 \mathrm{~Hz}, 1 \mathrm{H}, \mathrm{H}-3)$, 4.07-4.14 (m, 3H, H-6b, H-6'a, H-6'b), 4.22 (d, $J=8.1 \mathrm{~Hz}$, $\left.1 \mathrm{H}, \mathrm{H}-1^{\prime}\right), 4.62(\mathrm{~d}, J=3.5 \mathrm{~Hz}, 1 \mathrm{H}, \mathrm{H}-1), 4.63(\mathrm{~d}, J=11.1$ $\mathrm{Hz}, 1 \mathrm{H}, \mathrm{OCHPh}), 4.65$ (d, $J=12.4 \mathrm{~Hz}, 1 \mathrm{H}, \mathrm{OCHPh}), 4.76$ $\left(\mathrm{dd}, J=3.3,10.9 \mathrm{~Hz}, 1 \mathrm{H}, \mathrm{H}-3^{\prime}\right), 4.80(\mathrm{~d}, J=12.4 \mathrm{~Hz}, 1 \mathrm{H}$, OCHPh), $4.82(\mathrm{~d}, J=10.9 \mathrm{~Hz}, 1 \mathrm{H}, \mathrm{OCHPh}), 4.94(\mathrm{~d}, J=$ $11.1 \mathrm{~Hz}, 1 \mathrm{H}, \mathrm{OCHPh}), 4.99$ (d, $J=10.9 \mathrm{~Hz}, 1 \mathrm{H}, \mathrm{OCHPh})$, $5.30\left(\mathrm{~d}, J=3.3 \mathrm{~Hz}, 1 \mathrm{H}, \mathrm{H}-4^{\prime}\right), 7.27-7.37$ (m, 15H, Ar-H); ${ }^{13} \mathrm{C}$ NMR $\left(126 \mathrm{MHz}, \mathrm{CDCl}_{3}\right) \delta 20.56,20.58,20.61,55.3$, $60.9,61.1,66.3,68.8,69.7,70.6,71.3,73.4,75.7,77.7$, 79.8, 82.0, 98.2 (C-1), $102.4\left(\mathrm{C}-1^{\prime}\right), 127.6,127.7,127.8$, $127.9,128.0,128.1,128.4,128.45,128.46,138.1,138.3$, 138.7, 169.8, 170.0, 170.3; FAB-HRMS $\mathrm{m} / \mathrm{z}$ calcd for $\mathrm{C}_{40} \mathrm{H}_{47} \mathrm{~N}_{3} \mathrm{O}_{13} \mathrm{Na}(\mathrm{M}+\mathrm{Na})^{+}$800.3007, found 800.2985; Anal. calcd for: $\mathrm{C}_{40} \mathrm{H}_{47} \mathrm{~N}_{3} \mathrm{O}_{13}: \mathrm{C}, 61.77 ; \mathrm{H}, 6.09 ; \mathrm{N}, 5.40$, found $\mathrm{C}$, 61.75; H, 6.09; N, 5.30. Data for $\alpha$-anomer $(33 \alpha):{ }^{9}$ TLC $R_{f}$ $=0.43(1: 1$ hexane/AcOEt $), 0.31\left(1: 3\right.$ hexane/Et $\left.{ }_{2} \mathrm{O}\right) ;[\alpha]_{\mathrm{D}}{ }^{25}$ $+96.4^{\circ}$ (c 1.01, $\mathrm{CHCl}_{3}$ ); IR (film) 2928, 2110, 1751, 1454, 1371, 1228, $1074 \mathrm{~cm}^{-1} ;{ }^{1} \mathrm{H} \mathrm{NMR}\left(500 \mathrm{MHz}, \mathrm{CDCl}_{3}\right) \delta 1.98$ $\left(\mathrm{s}, 3 \mathrm{H}, \mathrm{CH}_{3} \mathrm{CO}\right), 2.05\left(\mathrm{~s}, 3 \mathrm{H}, \mathrm{CH}_{3} \mathrm{CO}\right), 2.12(\mathrm{~s}, 3 \mathrm{H}$, $\mathrm{CH}_{3} \mathrm{CO}$ ), 3.38 (s, $\left.3 \mathrm{H}, \mathrm{OCH}_{3}\right), 3.51$ (dd, $J=9.0,9.9 \mathrm{~Hz}, 1 \mathrm{H}$,
H-4), 3.52 (dd, $J=3.5,9.7 \mathrm{~Hz}, 1 \mathrm{H}, \mathrm{H}-2), 3.62$ (dd, $J=3.4$, $\left.11.2 \mathrm{~Hz}, 1 \mathrm{H}, \mathrm{H}-2^{\prime}\right), 3.70$ (m, 1H, H-6a), 3.75-3.79 (m, 2H, H-5, H-6b), 3.96-4.02 (m, 2H, H-3, H-6'a), 4.05 (dd, $J=$ 6.0, $\left.10.9 \mathrm{~Hz}, 1 \mathrm{H}, \mathrm{H}-6^{\prime} \mathrm{b}\right), 4.09$ (dd, $J=6.0,6.9 \mathrm{~Hz}, 1 \mathrm{H}, \mathrm{H}-$ $\left.5^{\prime}\right), 4.59(\mathrm{~d}, J=3.5 \mathrm{~Hz}, 1 \mathrm{H}, \mathrm{H}-1), 4.61(\mathrm{~d}, J=11.3 \mathrm{~Hz}, 1 \mathrm{H}$, OCHPh), $4.66(\mathrm{~d}, J=12.1 \mathrm{~Hz}, 1 \mathrm{H}, \mathrm{OCHPh}), 4.79$ (d, $J=$ $12.1 \mathrm{~Hz}, 1 \mathrm{H}, \mathrm{OCHPh}), 4.81$ (d, $J=10.9 \mathrm{~Hz}, 1 \mathrm{H}, \mathrm{OCHPh})$, $4.94(\mathrm{~d}, J=11.3 \mathrm{~Hz}, 1 \mathrm{H}, \mathrm{OCHPh}), 4.99$ (d, $J=10.9 \mathrm{~Hz}, 1 \mathrm{H}$, OCHPh), $5.06\left(\mathrm{~d}, J=3.6 \mathrm{~Hz}, 1 \mathrm{H}, \mathrm{H}^{\prime} 1^{\prime}\right), 5.28(\mathrm{dd}, J=3.3$, $11.2 \mathrm{~Hz}, 1 \mathrm{H}, \mathrm{H}-3^{\prime}$ ), 5.39 (brd, $J=3.3 \mathrm{~Hz}, 1 \mathrm{H}, \mathrm{H}-4^{\prime}$ ), 7.26-7.37 (m, 15H, Ar-H); ${ }^{13} \mathrm{C}$ NMR $\left(126 \mathrm{MHz}, \mathrm{CDCl}_{3}\right) \delta$ 20.5, 20.6, 55.2, 57.5, 61.6, 66.6, 66.7, 67.6, 68.0, 69.9, 73.4, 74.9, 75.7, 77.6, 80.0, 82.0, 97.9, 98.1, 127.6, 127.7, $127.85,127.94,128.1,128.3,128.4,138.1,138.2$, 138.6, 169.7, 169.9, 170.2; FAB-HRMS $\mathrm{m} / \mathrm{z}$ calcd for $\mathrm{C}_{40} \mathrm{H}_{47} \mathrm{~N}_{3} \mathrm{O}_{13} \mathrm{Na}(\mathrm{M}+\mathrm{Na})^{+} 800.3007$, found 800.3007.

\subsection{Glycosidations of 2 -azido-4,6- $O$-benzylidene-2- deoxyglycosyl diphenyl phosphates $2 \mathrm{c}$ and $4 \mathrm{c}$}

\subsubsection{Methyl 6-O-(3-O-acetyl-2-azido-4,6-O-benzyli- dene-2-deoxy-D-glucopyranosyl)-2,3,4-tri- $O$-benzyl- $\alpha$-D-} glucopyranoside (35). The glycosidation was performed according to the typical procedure $\left(1.5 \mathrm{~mL} \mathrm{EtCN},-45^{\circ} \mathrm{C}, 4\right.$ h) employing diphenyl phosphate $2 \mathrm{c}(56.7 \mathrm{mg}, 0.10 \mathrm{mmol})$, alcohol 7 (51.1 mg, 0.11 mmol), and TMSOTf (1.0 $\mathrm{M}$ in $\left.\mathrm{CH}_{2} \mathrm{Cl}_{2}, 0.15 \mathrm{~mL}, 0.15 \mathrm{mmol}\right)$. A mixture of disaccharide 35 and imidate $36(80.3 \mathrm{mg})$ was obtained as a colorless oil from the crude product (104.6 mg) after short column chromatography (silica gel $3 \mathrm{~g}, 3: 1$ hexane/AcOEt with $1 \%$ $\mathrm{Et}_{3} \mathrm{~N}$ ). The anomeric ratio of the products was determined by HPLC analysis [eluent, 4:1 hexane/AcOEt; flow rate, $1.0 \mathrm{~mL} / \mathrm{min} ; t_{\mathrm{R}}(36 \alpha)=17.4 \mathrm{~min}, t_{\mathrm{R}}(35 \beta)=21.5 \mathrm{~min}, t_{\mathrm{R}}$ $\left.(36 \beta)=25.4 \mathrm{~min}, t_{\mathrm{R}}(35 \alpha)=30.6 \mathrm{~min}\right]$. The mixture was purified by flash column chromatography (silica gel $8 \mathrm{~g}$, 5:1 hexane/AcOEt with $\left.1 \% \mathrm{Et}_{3} \mathrm{~N}\right)$ to give $\alpha$-imidate $\mathbf{3 6}$ (70.4 mg, 84\%) as a white amorphous, along with an anomeric mixture of disaccharide $35(5.0 \mathrm{mg}, 6 \%, \alpha: \beta=$ $8: 92)$ as a white solid. The $\alpha$ - and $\beta$-glycosides of disaccharide $\mathbf{3 5}$ were separated by flash column chromatography with 6:1 hexane/AcOEt. Data for $\beta$ anomer $(35 \beta)$ : TLC $R_{f}=0.38$ (2:1 hexane/AcOEt); mp 149.0-149.5 ${ }^{\circ} \mathrm{C}$ (colorless needles from AcOEt-hexane); $[\alpha]_{\mathrm{D}}{ }^{23}-35.5^{\circ}\left(c 1.01, \mathrm{CHCl}_{3}\right)$; IR (film) 2928, 2112, 1753, 1454, 1369, 1222, $1095 \mathrm{~cm}^{-1}$; ${ }^{1} \mathrm{H}$ NMR $\left(500 \mathrm{MHz}, \mathrm{CDCl}_{3}\right)$ $\delta 2.13\left(\mathrm{~s}, 3 \mathrm{H}, \mathrm{CH}_{3} \mathrm{CO}\right), 3.38\left(\mathrm{~s}, 3 \mathrm{H}, \mathrm{OCH}_{3}\right), 3.41$ (ddd, $J=$ 5.0, 9.4, $\left.10.1 \mathrm{~Hz}, 1 \mathrm{H}, \mathrm{H}-5^{\prime}\right), 3.53(\mathrm{dd}, J=8.0,9.8 \mathrm{~Hz}, 1 \mathrm{H}$, $\left.\mathrm{H}-2^{\prime}\right), 3.54(\mathrm{dd}, J=3.5,9.4 \mathrm{~Hz}, 1 \mathrm{H}, \mathrm{H}-2), 3.56(\mathrm{dd}, J=9.1$, $9.3 \mathrm{~Hz}, 1 \mathrm{H}, \mathrm{H}-4), 3.59$ (dd, $\left.J=9.4,9.7 \mathrm{~Hz}, 1 \mathrm{H}, \mathrm{H}-4^{\prime}\right), 3.75$ (dd, $J=4.2,10.4 \mathrm{~Hz}, 1 \mathrm{H}, \mathrm{H}-6 \mathrm{a}), 3.76$ (dd, $J=10.1,10.5$ $\mathrm{Hz}, 1 \mathrm{H}, \mathrm{H}-6$ 'ax), 3.80 (ddd, $J=1.4,4.2,9.3 \mathrm{~Hz}, 1 \mathrm{H}, \mathrm{H}-5$ ), 4.01 (dd, $J=9.1,9.4 \mathrm{~Hz}, 1 \mathrm{H}, \mathrm{H}-3), 4.08(\mathrm{dd}, J=1.4,10.4$ $\mathrm{Hz}, 1 \mathrm{H}, \mathrm{H}-6 \mathrm{~b}$ ), 4.30 (dd, $J=5.0,10.5 \mathrm{~Hz}, 1 \mathrm{H}, \mathrm{H}-6^{\prime}$ eq), $4.35\left(\mathrm{~d}, J=8.0 \mathrm{~Hz}, 1 \mathrm{H}, \mathrm{H}-1^{\prime}\right), 4.61(\mathrm{~d}, J=3.5 \mathrm{~Hz}, 1 \mathrm{H}, \mathrm{H}-1)$, $4.64(\mathrm{~d}, J=11.1 \mathrm{~Hz}, 1 \mathrm{H}, \mathrm{OCHPh}), 4.65$ (d, $J=12.2 \mathrm{~Hz}, 1 \mathrm{H}$, OCHPh), $4.80(\mathrm{~d}, J=12.2 \mathrm{~Hz}, 1 \mathrm{H}, \mathrm{OCHPh}), 4.83(\mathrm{~d}, J=$ $10.9 \mathrm{~Hz}, 1 \mathrm{H}, \mathrm{OC} H \mathrm{Ph}), 4.95(\mathrm{~d}, J=11.1 \mathrm{~Hz}, 1 \mathrm{H}, \mathrm{OCHPh})$, $4.99(\mathrm{~d}, J=10.9 \mathrm{~Hz}, 1 \mathrm{H}, \mathrm{OCHPh}), 5.15(\mathrm{dd}, J=9.7,9.8 \mathrm{~Hz}$, 1H, H-3'), 5.46 (s, 1H, CHPh), 7.28-7.36 (m, 18H, Ar-H), $7.41(\mathrm{~m}, 2 \mathrm{H}, \mathrm{Ar}-\mathrm{H}) ;{ }^{13} \mathrm{C} \mathrm{NMR}\left(126 \mathrm{MHz}, \mathrm{CDCl}_{3}\right) \delta 20.8$, 55.3, 64.8, 66.4, 68.4, 68.8, 69.6, 71.3, 73.4, 74.9, 75.7, 
77.6, 78.5, 79.7, 82.0, 98.2 (C-1), 101.5, $102.4\left(\mathrm{C}-1^{\prime}\right)$, $127.6,127.77,127.80,127.9,128.0,128.15,128.23,128.4$, $128.45,128.49,129.1,136.7,138.1,138.2,138.7,169.7$; FAB-HRMS $\mathrm{m} / \mathrm{z}$ calcd for $\mathrm{C}_{43} \mathrm{H}_{47} \mathrm{~N}_{3} \mathrm{O}_{11} \mathrm{Na} \quad(\mathrm{M}+\mathrm{Na})^{+}$ 804.3109, found 804.3135; Anal. calcd for: $\mathrm{C}_{43} \mathrm{H}_{47} \mathrm{~N}_{3} \mathrm{O}_{11}$ : C, 66.06; H, 6.06; N, 5.37, found C, 65.94; H, 6.13; N, 5.27. Data for $\alpha$-anomer $(\mathbf{3 5} \alpha)$ : TLC $R_{f}=0.32$ (2:1 hexane/ AcOEt); mp 152.0-153.0 ${ }^{\circ} \mathrm{C}$ (colorless fine needles from AcOEt-hexane); $[\alpha]_{\mathrm{D}}{ }^{21}+106.9^{\circ}\left(c 0.76, \mathrm{CHCl}_{3}\right)$; IR (film) 2926, 2112, 1753, 1454, 1369, 1222, $1095 \mathrm{~cm}^{-1}$; ${ }^{1} \mathrm{H}$ NMR $\left(500 \mathrm{MHz}, \mathrm{CDCl}_{3}\right) \delta 2.13\left(\mathrm{~s}, 3 \mathrm{H}, \mathrm{CH}_{3} \mathrm{CO}\right), 3.21(\mathrm{dd}, J=$ 3.6, $\left.10.4 \mathrm{~Hz}, 1 \mathrm{H}, \mathrm{H}-2^{\prime}\right), 3.39\left(3 \mathrm{H}, \mathrm{s}, \mathrm{OCH}_{3}\right), 3.54$ (dd, $J=$ $3.5,9.7 \mathrm{~Hz}, 1 \mathrm{H}, \mathrm{H}-2), 3.55$ (dd, $J=8.9,10.5 \mathrm{~Hz}, 1 \mathrm{H}, \mathrm{H}-5)$, $3.58\left(\mathrm{dd}, J=9.5,9.8 \mathrm{~Hz}, 1 \mathrm{H}, \mathrm{H}-4^{\prime}\right), 3.69$ (m, 1H, H-6a), 3.70 (dd, $\left.J=10.2,10.3 \mathrm{~Hz}, 1 \mathrm{H}, \mathrm{H}-6^{\prime} \mathrm{ax}\right), 3.77-3.83(\mathrm{~m}, 2 \mathrm{H}$, H-5, H-6b), 3.91 (ddd, $J=4.9,9.8,10.2 \mathrm{~Hz}, 1 \mathrm{H}, \mathrm{H}-5^{\prime}$ ), 4.01 (dd, $J=8.9,9.7 \mathrm{~Hz}, 1 \mathrm{H}, \mathrm{H}-3), 4.20(\mathrm{dd}, J=4.9,10.3$ $\left.\mathrm{Hz}, 1 \mathrm{H}, \mathrm{H}-6^{\prime} \mathrm{eq}\right), 4.59$ (d, $\left.J=3.5 \mathrm{~Hz}, 1 \mathrm{H}, \mathrm{H}-1\right), 4.63$ (d, $J=$ $11.2 \mathrm{~Hz}, 1 \mathrm{H}, \mathrm{OCHPh}), 4.65$ (d, $J=12.0 \mathrm{~Hz}, 1 \mathrm{H}, \mathrm{OCHPh})$, $4.77(\mathrm{~d}, J=12.0 \mathrm{~Hz}, 1 \mathrm{H}, \mathrm{OCHPh}), 4.82(\mathrm{~d}, J=11.0 \mathrm{~Hz}, 1 \mathrm{H}$, OCHPh), $4.95(\mathrm{~d}, J=11.2 \mathrm{~Hz}, 1 \mathrm{H}, \mathrm{OCHPh}), 4.99$ (d, $J=$ $11.0 \mathrm{~Hz}, 1 \mathrm{H}, \mathrm{OCHPh}), 5.02$ (d, $\left.J=3.6 \mathrm{~Hz}, 1 \mathrm{H}, \mathrm{H}-1^{\prime}\right), 5.48$ $(\mathrm{s}, 1 \mathrm{H}, \mathrm{CHPh}), 5.53\left(\mathrm{dd}, J=9.5,10.4 \mathrm{~Hz}, 1 \mathrm{H}, \mathrm{H}-3^{\prime}\right)$, 7.26-7.36 (m, 18H, Ar-H), $7.42(\mathrm{~m}, 2 \mathrm{H}, \mathrm{Ar}-\mathrm{H}) ;{ }^{13} \mathrm{C}$ NMR $\left(126 \mathrm{MHz}, \mathrm{CDCl}_{3}\right) \delta 20.9,55.3,61.8,62.7,66.9,68.7,68.8$, $69.9,73.5,75.1,75.7,77.5,79.5,80.0,82.1,98.1$ (C-1), $99.1\left(\mathrm{C}-1^{\prime}\right), 101.7,127.5,127.86,127.87,127.93,128.1$, 128.2, 128.37, 128.43, 128.5, 129.1, 136.9, 138.1, 138.8, 169.7; FAB-HRMS m/z calcd for $\mathrm{C}_{43} \mathrm{H}_{47} \mathrm{~N}_{3} \mathrm{O}_{11} \mathrm{Na}(\mathrm{M}+\mathrm{Na})^{+}$ 804.3109, found 804.3134.

Data for methyl 6-O-[1-(3-O-acetyl-2-azido-4,6- $O$-benzylidene-2-deoxy- $\alpha$-D-glucopyranosyl)iminopropyl]-2,3,4-tri$O$-benzyl- $\alpha$-D-glucopyranoside $(\mathbf{3 6} \alpha)$ : TLC $R_{f}=0.40$ (2:1 hexane/AcOEt); $[\alpha]_{\mathrm{D}}{ }^{20}+81.0^{\circ}$ (c 2.30, $\mathrm{CHCl}_{3}$ ); IR (film) 2926, 2106, 1753, 1660, 1454, 1369, 1224, $1095 \mathrm{~cm}^{-1} ;{ }^{1} \mathrm{H}$ NMR $\left(500 \mathrm{MHz}, \mathrm{CDCl}_{3}\right) \delta 1.14(\mathrm{t}, J=7.6 \mathrm{~Hz}, 3 \mathrm{H}$, $\mathrm{CH}_{2} \mathrm{CH}_{3}$ ), 2.15 (s, 3H, $\mathrm{CH}_{3} \mathrm{CO}$ ), 2.35 (q, $J=7.6 \mathrm{~Hz}, 2 \mathrm{H}$, $\mathrm{CH}_{2} \mathrm{CH}_{3}$ ), $3.397\left(\mathrm{dd}, J=4.2,10.0 \mathrm{~Hz}, 1 \mathrm{H}, \mathrm{H}-2^{\prime}\right), 3.399$ (s, $\left.3 \mathrm{H}, \mathrm{OCH}_{3}\right), 3.52(\mathrm{dd}, J=3.6,9.7 \mathrm{~Hz}, 1 \mathrm{H}, \mathrm{H}-2), 3.63(\mathrm{dd}, J$ $=8.8,10.5 \mathrm{~Hz}, 1 \mathrm{H}, \mathrm{H}-4), 3.64-3.70$ (m, 2H, H-4', H-6'ax), 3.88 (ddd, $J=1.6,4.1,10.5 \mathrm{~Hz}, 1 \mathrm{H}, \mathrm{H}-5), 4.00$ (dd, $J=8.8$, $9.7 \mathrm{~Hz}, 1 \mathrm{H}, \mathrm{H}-3), 4.14-4.20$ (m, 2H, H-5', H-6'eq), 4.23 (dd, $J=4.1,12.3 \mathrm{~Hz}, 1 \mathrm{H}, \mathrm{H}-6 \mathrm{a}), 4.53$ (dd, $J=1.6,12.3 \mathrm{~Hz}$, $1 \mathrm{H}, \mathrm{H}-6 \mathrm{~b}), 4.617$ (d, $J=10.5 \mathrm{~Hz}, 1 \mathrm{H}, \mathrm{OCHPh}), 4.619$ (d, $J$ $=12.1 \mathrm{~Hz}, 1 \mathrm{H}, \mathrm{OC} H \mathrm{Ph}), 4.64(\mathrm{~d}, J=3.6 \mathrm{~Hz}, 1 \mathrm{H}, \mathrm{H}-1)$, $4.74(\mathrm{~d}, J=12.1 \mathrm{~Hz}, 1 \mathrm{H}, \mathrm{OCHPh}), 4.83(\mathrm{~d}, J=10.8 \mathrm{~Hz}, 1 \mathrm{H}$, OCHPh), $4.84(\mathrm{~d}, J=10.5 \mathrm{~Hz}, 1 \mathrm{H}, \mathrm{OCHPh}), 4.97(\mathrm{~d}, J=$ $10.8 \mathrm{~Hz}, 1 \mathrm{H}, \mathrm{OCHPh}), 5.21\left(\mathrm{~d}, J=4.2 \mathrm{~Hz}, 1 \mathrm{H}, \mathrm{H}-1^{\prime}\right), 5.52$ (s, 1H, CHPh), $5.76\left(\mathrm{dd}, J=9.5,10.0 \mathrm{~Hz}, 1 \mathrm{H}, \mathrm{H}-3^{\prime}\right), 7.23$ $(\mathrm{m}, 1 \mathrm{H}, \mathrm{Ar}-\mathrm{H}), 7.25-7.36(\mathrm{~m}, 17 \mathrm{H}, \mathrm{Ar}-\mathrm{H}), 7.44(\mathrm{~m}, 2 \mathrm{H}$, $\mathrm{Ar}-\mathrm{H}) ;{ }^{13} \mathrm{C}$ NMR $\left(126 \mathrm{MHz}, \mathrm{CDCl}_{3}\right) \delta$ 10.7, 20.9, 23.0, 55.2, 62.7, 63.3, 64.4, 68.9, 69.2, 69.8, 73.2, 75.2, 75.8, 77.6, 80.1, 80.3, 82.1, 84.1 (C-1'), 97.9 (C-1), 101.5, 126.1, $127.6,127.79,127.83,128.0,128.1,128.2,128.38,128.40$, $128.5,129.0,137.1,138.0,138.2,138.7,169.7,170.4$; FAB-HRMS $m / z$ calcd for $\mathrm{C}_{46} \mathrm{H}_{53} \mathrm{~N}_{4} \mathrm{O}_{11}(\mathrm{M}+\mathrm{H})^{+} 837.3711$, found 837.3727; Anal. calcd for: $\mathrm{C}_{46} \mathrm{H}_{52} \mathrm{~N}_{4} \mathrm{O}_{11}: \mathrm{C}, 66.02 ; \mathrm{H}$, $6.26 ; \mathrm{N}, 6.69$, found $\mathrm{C}, 65.90 ; \mathrm{H}, 6.27 ; \mathrm{N}, 6.66$.
4.5.2. Methyl 6-O-(3-O-acetyl-2-azido-4,6- $O$-benzylidene-2-deoxy-D-galactopyranosyl)-2,3,4-tri- $O$-benzyl- $\alpha$ D-glucopyranoside (37). ${ }^{9}$ The glycosidation was performed according to the typical procedure $(1.5 \mathrm{~mL} \mathrm{EtCN}$, $\left.-78{ }^{\circ} \mathrm{C}, 3 \mathrm{~h}\right)$ employing diphenyl phosphate $4 \mathrm{c}(56.7 \mathrm{mg}$, $0.10 \mathrm{mmol}$ ), alcohol 7 (51.1 mg, $0.11 \mathrm{mmol})$, and TMSOTf (1.0 $\mathrm{M}$ in $\mathrm{CH}_{2} \mathrm{Cl}_{2}, 0.15 \mathrm{~mL}, 0.15 \mathrm{mmol}$ ). An anomeric mixture of disaccharide 37 (60.6 mg, 78\%, $\alpha: \beta=3: 97)$ was obtained as a white solid from the crude product $(98.4 \mathrm{mg}$ ) after column chromatography (silica gel $6 \mathrm{~g}, 40: 1 \rightarrow 30: 1$ $\mathrm{CH}_{2} \mathrm{Cl}_{2} / \mathrm{AcOEt}$ with $0.5 \% \mathrm{Et}_{3} \mathrm{~N}$ ), along with $\alpha$-imidate 38 $(8.5 \mathrm{mg}, 10 \%)$ as a colorless oil. The anomeric ratio of the product was determined by HPLC analysis [eluent, 4:1 hexane/AcOEt; flow rate, $1.0 \mathrm{~mL} / \mathrm{min} ; t_{\mathrm{R}}(\alpha$-anomer $)=$ $29.5 \mathrm{~min}, t_{\mathrm{R}}(\beta$-anomer $\left.)=79.6 \mathrm{~min}\right]$. The $\alpha$ - and $\beta$ glycosides were separated by flash column chromatography with $4: 1$ hexane/AcOEt. Data for $\beta$-anomer $(37 \beta):{ }^{9}$ TLC $R_{f}$ $=0.20(2: 1$ hexane/AcOEt $), 0.40\left(10: 1 \mathrm{CH}_{2} \mathrm{Cl}_{2} / \mathrm{AcOEt}\right) ; \mathrm{mp}$ 149.0-150.0 ${ }^{\circ} \mathrm{C}$ (colorless fine needles from AcOEthexane); $[\alpha]_{\mathrm{D}}^{23}+31.1^{\circ}$ (c 1.01, $\left.\mathrm{CHCl}_{3}\right)$; IR (KBr) 3032, 2918, 2114, 1745, 1454, 1367, 1246, $1059 \mathrm{~cm}^{-1}$; ${ }^{1} \mathrm{H}$ NMR $\left(500 \mathrm{MHz}, \mathrm{CDCl}_{3}\right) \delta 2.14$ (s, 3H, $\left.\mathrm{CH}_{3} \mathrm{CO}\right), 3.37$ (brs, $1 \mathrm{H}$, H-5') 3.39 (s, $3 \mathrm{H}, \mathrm{OCH}_{3}$ ), 3.53 (dd, $J=8.2,10.1 \mathrm{~Hz}, 1 \mathrm{H}$, $\mathrm{H}-4), 3.55$ (dd, $J=3.6,9.7 \mathrm{~Hz}, 1 \mathrm{H}, \mathrm{H}-2), 3.71(\mathrm{dd}, J=5.1$, $11.1 \mathrm{~Hz}, 1 \mathrm{H}, \mathrm{H}-6 \mathrm{a}), 3.85$ (ddd, $J=1.7,5.1,10.1 \mathrm{~Hz}, 1 \mathrm{H}, \mathrm{H}-$ 5), 3.95 (dd, $\left.J=8.0,10.7 \mathrm{~Hz}, 1 \mathrm{H}, \mathrm{H}-2^{\prime}\right), 3.99-4.02(\mathrm{~m}, 2 \mathrm{H}$, H-3, H-6'a), 4.16 (dd, $J=1.7,11.1 \mathrm{~Hz}, 1 \mathrm{H}, \mathrm{H}-6 \mathrm{~b}), 4.23$ (d, $\left.J=8.0 \mathrm{~Hz}, 1 \mathrm{H}, \mathrm{H}-1^{\prime}\right), 4.27-4.29$ (m, 2H, H-4', H-6'b), 4.62 $(\mathrm{d}, J=3.6 \mathrm{~Hz}, 1 \mathrm{H}, \mathrm{H}-1), 4.64$ (d, $J=11.1 \mathrm{~Hz}, 1 \mathrm{H}, \mathrm{OCHPh})$, $4.65(\mathrm{~d}, J=12.1 \mathrm{~Hz}, 1 \mathrm{H}, \mathrm{OCHPh}), 4.68(\mathrm{dd}, J=3.5,10.7$ $\left.\mathrm{Hz}, 1 \mathrm{H}, \mathrm{H}-3^{\prime}\right), 4.78$ (d, $\left.J=12.1 \mathrm{~Hz}, 1 \mathrm{H}, \mathrm{OCHPh}\right), 4.82$ (d, $J=11.0 \mathrm{~Hz}, 1 \mathrm{H}, \mathrm{OCHPh}), 4.93(\mathrm{~d}, J=11.1 \mathrm{~Hz}, 1 \mathrm{H}$, OCHPh), 4.99 (d, $J=11.0 \mathrm{~Hz}, 1 \mathrm{H}, \mathrm{OCHPh}), 5.48(\mathrm{~s}, 1 \mathrm{H}$, $\mathrm{CHPh}), 7.25-7.38$ (m, 18H, Ar-H), 7.47 (m, 2H, Ar-H); ${ }^{13} \mathrm{C}$ NMR $\left(126 \mathrm{MHz}, \mathrm{CDCl}_{3}\right) \delta$ 20.9, 55.3, 60.3, 66.3, 68.6, $68.8,69.9,72.5,72.6,73.4,74.9,75.7,77.9,79.9,82.1$, 98.1 (C-1), 100.9, 102.5 (C-1'), 126.2, 127.6, 127.7, 127.8, $127.9,128.0,128.1,128.2,128.36,128.44,129.1,137.5$, 138.1, 138.4, 138.8, 170.5; FAB-HRMS $\mathrm{m} / \mathrm{z}$ calcd for $\mathrm{C}_{43} \mathrm{H}_{47} \mathrm{~N}_{3} \mathrm{O}_{11} \mathrm{Na}(\mathrm{M}+\mathrm{Na})^{+}$804.3108, found 804.3094; Anal. calcd for: $\mathrm{C}_{43} \mathrm{H}_{47} \mathrm{~N}_{3} \mathrm{O}_{11}$ : C, 66.06; $\mathrm{H}, 6.06 ; \mathrm{N}, 5.37$, found $\mathrm{C}$, 66.07; H, 5.92; N, 5.41. Data for $\alpha$-anomer $(37 \alpha):{ }^{9}$ TLC $R_{f}$ $=0.34$ (2:1 hexane/AcOEt), 0.53 (10:1 $\left.\mathrm{CH}_{2} \mathrm{Cl}_{2} / \mathrm{AcOEt}\right)$; $[\alpha]_{\mathrm{D}}^{22}+153.1^{\circ}\left(c 1.00, \mathrm{CHCl}_{3}\right) ; \mathrm{IR}(\mathrm{KBr}) 3032,2914,2110$, $1745,1496,1454,1371,1228,1145,1028 \mathrm{~cm}^{-1}$; ${ }^{1} \mathrm{H}$ NMR $\left(500 \mathrm{MHz}, \mathrm{CDCl}_{3}\right) \delta 2.14\left(\mathrm{~s}, 3 \mathrm{H}, \mathrm{CH}_{3} \mathrm{CO}\right), 3.38(\mathrm{~s}, 3 \mathrm{H}$, $\mathrm{OCH}_{3}$ ), 3.52-3.56 (m, 2H, H-2, H-4), 3.56 (brs, 1H, H-5'), 3.70 (m, 1H, H-6a), 3.77-3.81 (m, 2H, H-5, H-6b), 3.88 (d, $J=12.5 \mathrm{~Hz}, 1 \mathrm{H}, \mathrm{OCHPh}), 3.90(\mathrm{dd}, J=3.1,11.0 \mathrm{~Hz}, 1 \mathrm{H}$, H-2'), 4.01 (t, $J=9.2 \mathrm{~Hz}, 1 \mathrm{H}, \mathrm{H}-3), 4.13$ (d, $J=12.5 \mathrm{~Hz}$, $1 \mathrm{H}, \mathrm{OCHPh}), 4.38\left(\mathrm{~d}, J=3.1 \mathrm{~Hz}, 1 \mathrm{H}, \mathrm{H}-4^{\prime}\right), 4.59(\mathrm{~d}, J=$ $3.6 \mathrm{~Hz}, 1 \mathrm{H}, \mathrm{H}-1), 4.60$ (d, $J=11.6 \mathrm{~Hz}, 1 \mathrm{H}, \mathrm{OCHPh}), 4.66$ $(\mathrm{d}, J=12.0 \mathrm{~Hz}, 1 \mathrm{H}, \mathrm{OCHPh}), 4.79(\mathrm{~d}, J=12.0 \mathrm{~Hz}, 1 \mathrm{H}$, OCHPh), $4.81(\mathrm{~d}, J=10.9 \mathrm{~Hz}, 1 \mathrm{H}, \mathrm{OCHPh}), 4.96(\mathrm{~d}, J=$ $11.6 \mathrm{~Hz}, 1 \mathrm{H}, \mathrm{OCHPh}), 4.99$ (d, $J=10.9 \mathrm{~Hz}, 1 \mathrm{H}, \mathrm{OCHPh})$, $5.12\left(\mathrm{~d}, J=3.1 \mathrm{~Hz}, 1 \mathrm{H}, \mathrm{H}-1^{\prime}\right), 5.22(\mathrm{dd}, J=3.1,11.0 \mathrm{~Hz}$, 1H, H-3'), 5.47 (s, 1H, CHPh), 7.27-7.36 (m, 18H, Ar-H), $7.47(\mathrm{~m}, 2 \mathrm{H}, \mathrm{Ar}-\mathrm{H}) ;{ }^{13} \mathrm{C}$ NMR $\left(126 \mathrm{MHz}, \mathrm{CDCl}_{3}\right) \delta 20.9$, 55.1, 57.2, 62.3, 66.6, 69.0, 69.3, 69.9, 73.3, 73.4, 74.8, 75.7, 77.8, 80.0, 82.0, 98.0 (C-1), 98.6 (C-1'), 100.7, 126.1, 127.56, 127.63, 127.9, 128.0, 128.07, 128.14, 128.3, 128.4, 
129.0, 137.5, 138.1, 138.4, 138.7, 170.5; FAB-HRMS m/z calcd for $\mathrm{C}_{43} \mathrm{H}_{47} \mathrm{~N}_{3} \mathrm{O}_{11} \mathrm{Na} \quad(\mathrm{M}+\mathrm{Na})^{+}$804.3108, found 804.3093.

Data for methyl 6-O-[1-(3- $O$-acetyl-2-azido-4,6- $O$-benzylidene-2-deoxy- $\alpha$-D-galactopyranosyl)iminopropyl]-2,3,4tri- $O$-benzyl- $\alpha$-D-glucopyranoside (38): TLC $R_{f}=0.34$ (2:1 hexane/AcOEt); $[\alpha]_{\mathrm{D}}^{22}+104.2^{\circ}\left(\right.$ c $\left.0.31, \mathrm{CHCl}_{3}\right) ; \mathrm{IR}$ (film) 3032, 2908, 2108, 1743, 1662, 1454, 1373, 1228, 1095 $\mathrm{cm}^{-1}$; ${ }^{1} \mathrm{H}$ NMR $\left(500 \mathrm{MHz}, \mathrm{CDCl}_{3}\right) \delta 1.14(\mathrm{t}, J=7.6 \mathrm{~Hz}, 3 \mathrm{H}$, $\left.\mathrm{CH}_{2} \mathrm{CH}_{3}\right), 2.15$ (s, $3 \mathrm{H}, \mathrm{CH}_{3} \mathrm{CO}$ ), 2.36 (q, $J=7.6 \mathrm{~Hz}, 2 \mathrm{H}$, $\left.\mathrm{CH}_{2} \mathrm{CH}_{3}\right), 3.39\left(\mathrm{~s}, 3 \mathrm{H}, \mathrm{OCH}_{3}\right), 3.55(\mathrm{dd}, J=3.6,9.6 \mathrm{~Hz}, 1 \mathrm{H}$, $\mathrm{H}-2), 3.59$ (dd, $J=8.9,10.1 \mathrm{~Hz}, 1 \mathrm{H}, \mathrm{H}-4), 3.87$ (ddd, $J=$ 1.7, 4.5, $10.1 \mathrm{~Hz}, 1 \mathrm{H}, \mathrm{H}-5), 3.92$ (brs, $1 \mathrm{H}, \mathrm{H}-5^{\prime}$ ), 3.94 (dd, $J$ $\left.=1.6,12.6 \mathrm{~Hz}, 1 \mathrm{H}, \mathrm{H}-6^{\prime} \mathrm{a}\right), 4.01(\mathrm{dd}, J=8.9,9.6 \mathrm{~Hz}, 1 \mathrm{H}$, $\mathrm{H}-3), 4.09$ (dd, $\left.J=4.0,10.9 \mathrm{~Hz}, 1 \mathrm{H}, \mathrm{H}-2^{\prime}\right), 4.12$ (dd, $J=$ $\left.1.5,12.6 \mathrm{~Hz}, 1 \mathrm{H}, \mathrm{H}-6^{\prime} \mathrm{b}\right), 4.20$ (dd, $J=4.5,12.2 \mathrm{~Hz}, 1 \mathrm{H}, \mathrm{H}-$ 6a), 4.36 (dd, $J=1.7,12.2 \mathrm{~Hz}, 1 \mathrm{H}, \mathrm{H}-6 \mathrm{~b}), 4.43$ (d, $J=3.5$ $\left.\mathrm{Hz}, 1 \mathrm{H}, \mathrm{H}-4^{\prime}\right), 4.59$ (d, J = 11.0 Hz, 1H, OCHPh), 4.60 (d, $J=3.6 \mathrm{~Hz}, 1 \mathrm{H}, \mathrm{H}-1), 4.67(\mathrm{~d}, J=12.0 \mathrm{~Hz}, 1 \mathrm{H}, \mathrm{OCHPh})$, $4.81(\mathrm{~d}, J=12.0 \mathrm{~Hz}, 1 \mathrm{H}, \mathrm{OCHPh}), 4.84(\mathrm{~d}, J=10.8 \mathrm{~Hz}, 1 \mathrm{H}$, OCHPh), $4.86(\mathrm{~d}, J=11.0 \mathrm{~Hz}, 1 \mathrm{H}, \mathrm{OCHPh}), 4.99$ (d, $J=$ $10.8 \mathrm{~Hz}, 1 \mathrm{H}, \mathrm{OC} H \mathrm{Ph}), 5.33\left(\mathrm{~d}, J=4.0 \mathrm{~Hz}, 1 \mathrm{H}, \mathrm{H}-1^{\prime}\right), 5.41$ $\left(\mathrm{dd}, J=3.5,10.9 \mathrm{~Hz}, 1 \mathrm{H}, \mathrm{H}-3^{\prime}\right), 5.50(\mathrm{~s}, 1 \mathrm{H}, \mathrm{CHPh})$, 7.26-7.39 (m, 18H, Ar-H), 7.51 (m, 2H, Ar-H); ${ }^{13} \mathrm{C}$ NMR $\left(126 \mathrm{MHz}, \mathrm{CDCl}_{3}\right) \delta 10.8,21.1,22.9,55.2,58.3,62.9,64.4$, $68.8,69.5,70.7,73.4,73.7,75.2,75.9,77.9,80.0,82.1$, $83.6\left(\mathrm{C}-1^{\prime}\right), 98.2$ (C-1), 100.8, 126.2, 127.66, 127.73, 127.9, 127.97, 128.04, 128.1, 128.2, 128.4, 128.5, 129.0, 137.7, 138.1, 138.2, 138.7, 169.4, 170.6; FAB-HRMS $\mathrm{m} / \mathrm{z}$ calcd for $\mathrm{C}_{46} \mathrm{H}_{53} \mathrm{~N}_{4} \mathrm{O}_{11}(\mathrm{M}+\mathrm{H})^{+}$837.3711, found 837.3692.

4.5.3. Methyl 4-O-(3-O-acetyl-2-azido-4,6- $O$-benzylidene-2-deoxy-D-galactopyranosyl)-2,3,6-tri- $O$-benzyl- $\alpha$ D-glucopyranoside (39). The glycosidation was performed according to the typical procedure $\left(1.5 \mathrm{~mL} \mathrm{EtCN},-78^{\circ} \mathrm{C}, 3\right.$ h) employing diphenyl phosphate $4 \mathrm{c}(56.7 \mathrm{mg}, 0.10 \mathrm{mmol})$, alcohol 8 (51.1 mg, $0.11 \mathrm{mmol})$, and TMSOTf $(1.0 \mathrm{M}$ in $\left.\mathrm{CH}_{2} \mathrm{Cl}_{2}, 0.15 \mathrm{~mL}, 0.15 \mathrm{mmol}\right)$. An anomeric mixture of disaccharide 39 (70.1 mg, 90\%, $\alpha: \beta=4: 96)$ was obtained as a colorless oil from the crude product $(97.7 \mathrm{mg})$ after column chromatography (silica gel $6 \mathrm{~g}, \quad 15: 1$ toluene/ AcOEt). The anomeric ratio of the product was determined by HPLC analysis [eluent, 4:1 hexane/AcOEt; flow rate, $1.0 \mathrm{~mL} / \mathrm{min} ; t_{\mathrm{R}}(\alpha$-anomer $)=28.6 \mathrm{~min}, t_{\mathrm{R}}(\beta$-anomer $)=$ $60.0 \mathrm{~min}]$. The $\alpha$ - and $\beta$-glycosides were separated by flash column chromatography with $3: 1$ hexane/AcOEt. Data for $\beta$-anomer $(39 \beta)$ : TLC $R_{f}=0.23$ (2:1 hexane/AcOEt), 0.21 $\left(5: 1\right.$ toluene/AcOEt); $[\alpha]_{\mathrm{D}}{ }^{27}+24.2^{\circ}$ (c 1.17, $\left.\mathrm{CHCl}_{3}\right)$; IR (film) 3032, 2903, 2114, 1747, 1454, 1367, 1232, 1047 , $912 \mathrm{~cm}^{-1}$; ${ }^{1} \mathrm{H}$ NMR $\left(500 \mathrm{MHz}, \mathrm{CDCl}_{3}\right) \delta 2.14(\mathrm{~s}, 3 \mathrm{H}$, $\mathrm{CH}_{3} \mathrm{CO}$ ), 2.97 (brs, $1 \mathrm{H}, \mathrm{H}-5^{\prime}$ ), 3.39 (s, $3 \mathrm{H}, \mathrm{OCH}_{3}$ ), 3.53 (dd, $J=3.6,9.4 \mathrm{~Hz}, 1 \mathrm{H}, \mathrm{H}-2), 3.72(\mathrm{dd}, J=1.6,10.8 \mathrm{~Hz}, 1 \mathrm{H}$, H-6a), 3.78 (m, 1H, H-5), 3.81 (dd, $J=8.1,10.7 \mathrm{~Hz}, 1 \mathrm{H}$, H-2'), 3.85 (dd, $J=1.7,12.5 \mathrm{~Hz}, 1 \mathrm{H}, \mathrm{H}-6$ ' a), 3.92 (dd, $J=$ 9.0, $9.4 \mathrm{~Hz}, 1 \mathrm{H}, \mathrm{H}-3$ ), 3.98 (dd, $J=9.0,9.8 \mathrm{~Hz}, 1 \mathrm{H}, \mathrm{H}-4$ ), 3.99 (dd, $J=2.5,10.8 \mathrm{~Hz}, 1 \mathrm{H}, \mathrm{H}-6 \mathrm{~b}), 4.192$ (dd, $J=1.0$, $\left.12.5 \mathrm{~Hz}, 1 \mathrm{H}, \mathrm{H}-6^{\prime} \mathrm{b}\right), 4.194$ (d, $\left.J=3.7 \mathrm{~Hz}, 1 \mathrm{H}, \mathrm{H}-4^{\prime}\right), 4.25$ $\left(\mathrm{d}, J=8.1 \mathrm{~Hz}, 1 \mathrm{H}, \mathrm{H}-1^{\prime}\right), 4.42(\mathrm{~d}, J=12.1 \mathrm{~Hz}, 1 \mathrm{H}$, OCHPh), 4.47 (dd, $\left.J=3.7,10.7 \mathrm{~Hz}, 1 \mathrm{H}, \mathrm{H}-3^{\prime}\right), 4.60$ (d, $J=$ $3.6 \mathrm{~Hz}, 1 \mathrm{H}, \mathrm{H}-1), 4.63$ (d, $J=12.1 \mathrm{~Hz}, 1 \mathrm{H}, \mathrm{OCHPh}), 4.72$ $(\mathrm{d}, J=12.1 \mathrm{~Hz}, 1 \mathrm{H}, \mathrm{OCHPh}), 4.78(\mathrm{~d}, J=10.6 \mathrm{~Hz}, 1 \mathrm{H}$, OCHPh), $4.81(\mathrm{~d}, J=12.1 \mathrm{~Hz}, 1 \mathrm{H}, \mathrm{OCHPh}), 5.10(\mathrm{~d}, J=$ $10.6 \mathrm{~Hz}, 1 \mathrm{H}, \mathrm{OCHPh}), 5.46$ (s, 1H, CHPh), 7.17-7.22 (m, 3H, Ar-H), 7.25-7.35 (m, 13H, Ar-H), 7.45-7.47 (m, 4H, $\mathrm{Ar}-\mathrm{H}) ;{ }^{13} \mathrm{C} \mathrm{NMR}\left(126 \mathrm{MHz}, \mathrm{CDCl}_{3}\right) \delta$ 20.9, 55.3, 60.9, $66.2,68.2,68.6,69.7,72.5,72.8,73.4,73.6,75.9,77.4$, 79.2, 80.3, $98.3(\mathrm{C}-1), 100.9,101.2\left(\mathrm{C}-1^{\prime}\right), 127.8,127.9$, $128.06,128.08,128.10,128.11,128.2,128.35,128.40$, $128.5,129.0,137.7,138.0,138.4,139.1,170.4$; FABHRMS $\mathrm{m} / \mathrm{z}$ calcd for $\mathrm{C}_{43} \mathrm{H}_{48} \mathrm{~N}_{3} \mathrm{O}_{11}(\mathrm{M}+\mathrm{H})^{+} 782.3289$, found 782.3281; Anal. calcd for: $\mathrm{C}_{43} \mathrm{H}_{47} \mathrm{~N}_{3} \mathrm{O}_{11}$ : C, 66.06; $\mathrm{H}, 6.06$; $\mathrm{N}, 5.37$, found $\mathrm{C}, 65.94 ; \mathrm{H}, 6.13 ; \mathrm{N}, 5.27$. Data for $\alpha-$ anomer $(39 \alpha)$ : TLC $R_{f}=0.35$ (2:1 hexane/AcOEt), 0.38 $\left(5: 1\right.$ toluene/AcOEt); $[\alpha]_{\mathrm{D}}{ }^{24}+99.5^{\circ}$ (c 1.24, $\left.\mathrm{CHCl}_{3}\right)$; IR (film) 3032, 2908, 2110, 1743, 1496, 1454, 1369, 1228, 1143, 1101, $1039 \mathrm{~cm}^{-1} ;{ }^{1} \mathrm{H}$ NMR (500 MHz, $\left.\mathrm{CDCl}_{3}\right) \delta 2.14$ (s, $\left.3 \mathrm{H}, \mathrm{CH}_{3} \mathrm{CO}\right), 3.40\left(\mathrm{~s}, 3 \mathrm{H}, \mathrm{OCH}_{3}\right), 3.54$ (brs, $\left.1 \mathrm{H}, \mathrm{H}-5^{\prime}\right)$, 3.57 (dd, $J=3.5,9.6 \mathrm{~Hz}, 1 \mathrm{H}, \mathrm{H}-2), 3.61(\mathrm{dd}, J=1.2,12.6$ Hz, 1H, H-6'a), 3.63 (dd, $J=1.4,11.2 \mathrm{~Hz}, 1 \mathrm{H}, \mathrm{H}-6 \mathrm{a}), 3.75$ (dd, $J=4.2,11.2 \mathrm{~Hz}, 1 \mathrm{H}, \mathrm{H}-6 \mathrm{~b}$ ), 3.80 (ddd, $J=1.4,4.2,9.8$ $\mathrm{Hz}, 1 \mathrm{H}, \mathrm{H}-5), 3.85$ (dd, $\left.J=3.6,11.2 \mathrm{~Hz}, 1 \mathrm{H}, \mathrm{H}-2^{\prime}\right), 3.87$ (brd, $\left.J=12.6 \mathrm{~Hz}, 1 \mathrm{H}, \mathrm{H}-6^{\prime} \mathrm{b}\right), 3.94$ (dd, $J=8.7,9.8 \mathrm{~Hz}, 1 \mathrm{H}$, H-4), 4.08 (dd, $J=8.7,9.6 \mathrm{~Hz}, 1 \mathrm{H}, \mathrm{H}-3), 4.29$ (d, $J=3.3$ $\left.\mathrm{Hz}, 1 \mathrm{H}, \mathrm{H}-4^{\prime}\right), 4.53$ (d, $\left.J=12.2 \mathrm{~Hz}, 1 \mathrm{H}, \mathrm{OCHPh}\right), 4.59$ (d, $J=12.2 \mathrm{~Hz}, 1 \mathrm{H}, \mathrm{OCHPh}), 4.60(\mathrm{~d}, J=12.0 \mathrm{~Hz}, 1 \mathrm{H}$, $\mathrm{OCHPh}), 4.61(\mathrm{~d}, J=3.5 \mathrm{~Hz}, 1 \mathrm{H}, \mathrm{H}-1), 4.73(\mathrm{~d}, J=12.0$ $\mathrm{Hz}, 1 \mathrm{H}, \mathrm{OCHPh}), 4.85$ (d, $J=10.9 \mathrm{~Hz}, 1 \mathrm{H}, \mathrm{OCHPh}), 5.10$ $(\mathrm{d}, J=10.9 \mathrm{~Hz}, 1 \mathrm{H}, \mathrm{OC} H \mathrm{Ph}), 5.20(\mathrm{dd}, J=3.3,11.2 \mathrm{~Hz}$, $\left.1 \mathrm{H}, \mathrm{H}-3^{\prime}\right), 5.38(\mathrm{~s}, 1 \mathrm{H}, \mathrm{CHPh}), 5.85(\mathrm{~d}, J=3.6 \mathrm{~Hz}, 1 \mathrm{H}, \mathrm{H}-$ $\left.1^{\prime}\right), 7.26-7.38$ (m, 18H, Ar-H), $7.43(\mathrm{~m}, 2 \mathrm{H}, \mathrm{Ar}-\mathrm{H}) ;{ }^{13} \mathrm{C}$ NMR $\left(126 \mathrm{MHz}, \mathrm{CDCl}_{3}\right) \delta$ 21.0, 55.3, 57.0, 62.7, 69.0, $69.1,69.45,69.48,73.1,73.3,73.4,73.5,74.8,80.6,81.9$, $97.8,98.0,100.6,126.1,127.3,127.36,127.39,127.7$, $128.0,128.1,128.2,128.3,128.48,128.50,129.0,137.5$, 137.9, 138.0, 138.7, 170.5; FAB-HRMS $\mathrm{m} / \mathrm{z}$ calcd for $\mathrm{C}_{43} \mathrm{H}_{48} \mathrm{~N}_{3} \mathrm{O}_{11}(\mathrm{M}+\mathrm{H})^{+} 782.3289$, found 782.3306 .

\subsubsection{Allyl 3-O-(3-O-acetyl-2-azido-4,6- $O$-benzylidene- 2-deoxy-D-galactopyranosyl)-4,6- $O$-benzylidene- $\beta$-D-} galactopyranoside $\mathbf{( 4 0 )} .^{22 b}$ The glycosidation was performed according to the typical procedure $[1.55 \mathrm{~mL}$ EtCN- $\mathrm{CH}_{2} \mathrm{Cl}_{2}(30: 1),-78{ }^{\circ} \mathrm{C}, 2 \mathrm{~h}$ ] employing diphenyl phosphate 4c $(56.7 \mathrm{mg}, 0.10 \mathrm{mmol})$, alcohol $34(33.9 \mathrm{mg}$, $0.11 \mathrm{mmol}$ ), and TMSOTf (1.0 $\mathrm{M}$ in $\mathrm{CH}_{2} \mathrm{Cl}_{2}, 0.15 \mathrm{~mL}, 0.15$ $\mathrm{mmol})$. An anomeric mixture of disaccharide $\mathbf{4 0}(50.1 \mathrm{mg}$, $80 \%, \alpha: \beta=1: 99$ ) was obtained as a colorless film from the crude product $(87.4 \mathrm{mg})$ after column chromatography (silica gel $6 \mathrm{~g}, 5: 1$ toluene/AcOEt). The anomeric ratio of the product was determined by HPLC analysis [eluent, 1:1.5 hexane/AcOEt; flow rate, $1.0 \mathrm{~mL} / \mathrm{min} ; t_{\mathrm{R}}(\alpha$-anomer) $=9.4 \mathrm{~min}, t_{\mathrm{R}}(\beta$-anomer $\left.)=14.6 \mathrm{~min}\right]$. The $\alpha$ - and $\beta$ glycosides were separated by flash column chromatography with $1: 1$ hexane/AcOEt. Data for $\beta$-anomer $(\mathbf{4 0} \beta):{ }^{22 \mathrm{~b}}$ TLC $R_{f}=0.36$ (1:3 hexane/AcOEt), 0.36 (3:1 toluene/acetone); $[\alpha]_{\mathrm{D}}{ }^{21}+24.2^{\circ}\left(c 1.00, \mathrm{CHCl}_{3}\right) ; \mathrm{IR}(\mathrm{KBr}) 3514,2870,2116$, 1745, 1454, 1369, 1236, 1051, $916 \mathrm{~cm}^{-1} ;{ }^{1} \mathrm{H}$ NMR (500 $\left.\mathrm{MHz}, \mathrm{CDCl}_{3}\right) \delta 2.13$ (s, 3H, $\mathrm{CH}_{3} \mathrm{CO}$ ), 3.03 (br, $\left.1 \mathrm{H}, \mathrm{OH}\right)$, 3.40 (brs, $1 \mathrm{H}, \mathrm{H}-5), 3.46$ (brs, $\left.1 \mathrm{H}, \mathrm{H}-5^{\prime}\right), 3.86$ (dd, $J=3.5$, $10.0 \mathrm{~Hz}, 1 \mathrm{H}, \mathrm{H}-3), 3.99$ (dd, $J=8.0,10.8 \mathrm{~Hz}, 1 \mathrm{H}, \mathrm{H}-2^{\prime}$ ), $4.03\left(\mathrm{dd}, J=1.5,12.5 \mathrm{~Hz}, 1 \mathrm{H}, \mathrm{H}-6^{\prime} \mathrm{a}\right), 4.05$ (dd, $J=1.1$, $12.5 \mathrm{~Hz}, 1 \mathrm{H}, \mathrm{H}-6 \mathrm{a}), 4.11$ (dd, $J=7.8,10.0 \mathrm{~Hz}, 1 \mathrm{H}, \mathrm{H}-2$ ), 
$4.14\left(\mathrm{~m}, 1 \mathrm{H}, \mathrm{CHCH}=\mathrm{CH}_{2}\right), 4.27$ (brd, $J=12.5 \mathrm{~Hz}, 1 \mathrm{H}, \mathrm{H}-$ $\left.6^{\prime} \mathrm{b}\right), 4.28\left(\mathrm{~d}, J=3.6 \mathrm{~Hz}, 1 \mathrm{H}, \mathrm{H}-4^{\prime}\right), 4.31$ (brd, $J=12.5 \mathrm{~Hz}$, $1 \mathrm{H}, \mathrm{H}-6 \mathrm{~b}), 4.37$ (d, $J=7.8 \mathrm{~Hz}, 1 \mathrm{H}, \mathrm{H}-1), 4.38$ (d, $J=3.5$ $\mathrm{Hz}, 1 \mathrm{H}, \mathrm{H}-4), 4.42\left(\mathrm{~m}, 1 \mathrm{H}, \mathrm{CHCH}=\mathrm{CH}_{2}\right), 4.73(\mathrm{dd}, J=3.6$, $\left.10.8 \mathrm{~Hz}, 1 \mathrm{H}, \mathrm{H}-3^{\prime}\right), 4.98\left(1 \mathrm{H}, \mathrm{d}, J=8.0 \mathrm{~Hz}, \mathrm{H}-1^{\prime}\right), 5.20(1 \mathrm{H}$, $\left.\mathrm{dd}, J=0.9,10.6 \mathrm{~Hz}, \mathrm{CH}_{2} \mathrm{CH}=\mathrm{CH}\right), 5.32(1 \mathrm{H}, \mathrm{dd}, J=1.2$, $\left.17.3 \mathrm{~Hz}, \mathrm{CH}_{2} \mathrm{CH}=\mathrm{CH}\right), 5.49(\mathrm{~s}, 1 \mathrm{H}, \mathrm{CHPh}), 5.57(\mathrm{~s}, 1 \mathrm{H}$, $\mathrm{CHPh}), 5.95\left(\mathrm{~m}, 1 \mathrm{H}, \mathrm{CH}_{2} \mathrm{CH}=\mathrm{CH}_{2}\right), 7.28-7.37(\mathrm{~m}, 6 \mathrm{H}, \mathrm{Ar}-$ $\mathrm{H}), 7.49(\mathrm{~m}, 2 \mathrm{H}$, Ar- $\mathrm{H}), 7.54(\mathrm{~m}, 2 \mathrm{H}, \mathrm{Ar}-\mathrm{H}) ;{ }^{13} \mathrm{C}$ NMR $\left(100 \mathrm{MHz}, \mathrm{CDCl}_{3}\right) \delta 21.0,60.1,66.2,66.6,68.9,69.0,69.9$, $70.2,71.9,72.5,75.9,78.9,100.5,100.7,101.6(\mathrm{C}-1)$, $102.0\left(\mathrm{C}-1^{\prime}\right), 117.8,126.06,126.09,127.9,128.1,128.5$, 129.0, 133.7, 137.4, 137.6, 170.3; FAB-HRMS m/z calcd for $\mathrm{C}_{31} \mathrm{H}_{36} \mathrm{~N}_{3} \mathrm{O}_{11}(\mathrm{M}+\mathrm{H})^{+}$626.2350, found 626.2353; Anal. calcd for: $\mathrm{C}_{31} \mathrm{H}_{35} \mathrm{~N}_{3} \mathrm{O}_{11}: \mathrm{C}, 59.51 ; \mathrm{H}, 5.64 ; \mathrm{N}, 6.72$, found $\mathrm{C}$, 59.32; H, 5.64; N, 6.62. Data for $\alpha$-anomer $(40 \alpha)$ : TLC $R_{f}$ $=0.55$ (1:3 hexane/AcOEt), 0.44 (3:1 toluene/acetone); $[\alpha]_{\mathrm{D}}^{23}+165.1^{\circ}\left(c 0.36, \mathrm{CHCl}_{3}\right)$; IR (film) $3510,2922,2864$, 2110, 1743, 1452, 1369, 1244, $1049 \mathrm{~cm}^{-1} ;{ }^{1} \mathrm{H}$ NMR (500 $\left.\mathrm{MHz}, \mathrm{CDCl}_{3}\right) \delta 2.14$ (s, 3H, CH $\mathrm{CO}$ ), 2.63 (br, $1 \mathrm{H}, \mathrm{OH}$ ), 3.44 (brs, $1 \mathrm{H}, \mathrm{H}-5), 3.80$ (dd, $J=3.7,9.8 \mathrm{~Hz}, 1 \mathrm{H}, \mathrm{H}-3)$, $3.93\left(\mathrm{dd}, J=3.4,11.2 \mathrm{~Hz}, 1 \mathrm{H}, \mathrm{H}-2^{\prime}\right), 3.98$ (dd, $J=7.8,9.8$ $\mathrm{Hz}, 1 \mathrm{H}, \mathrm{H}-2), 4.04$ (dd, $\left.J=1.1,12.6 \mathrm{~Hz}, 1 \mathrm{H}, \mathrm{H}-6^{\prime} \mathrm{a}\right), 4.11$ $(\mathrm{dd}, J=1.1,12.5 \mathrm{~Hz}, 1 \mathrm{H}, \mathrm{H}-6 \mathrm{a}), 4.14(\mathrm{~m}, 1 \mathrm{H}$, $\mathrm{CHCH}=\mathrm{CH}_{2}$ ), 4.19 (brs, $\left.1 \mathrm{H}, \mathrm{H}-5^{\prime}\right), 4.24$ (dd, $J=1.2,12.6$ $\left.\mathrm{Hz}, 1 \mathrm{H}, \mathrm{H}-6^{\prime} \mathrm{b}\right), 4.32$ (d, $\left.J=3.7 \mathrm{~Hz}, 1 \mathrm{H}, \mathrm{H}-4\right), 4.36$ (d, $J=$ $7.8 \mathrm{~Hz}, 1 \mathrm{H}, \mathrm{H}-1), 4.37$ (dd, $J=1.3,12.5 \mathrm{~Hz}, 1 \mathrm{H}, \mathrm{H}-6 \mathrm{~b})$, 4.44 (dddd, $J=1.0,1.1,5.1,12.7 \mathrm{~Hz}, 1 \mathrm{H}, \mathrm{CHCH}=\mathrm{CH}_{2}$ ), $4.50\left(\mathrm{~d}, J=3.3 \mathrm{~Hz}, 1 \mathrm{H}, \mathrm{H}-4^{\prime}\right), 5.23(\mathrm{dd}, J=1.0,11.1 \mathrm{~Hz}$, $\left.1 \mathrm{H}, \mathrm{CH}_{2} \mathrm{CH}=\mathrm{CH}\right), 5.30\left(\mathrm{~d}, J=3.4 \mathrm{~Hz}, 1 \mathrm{H}, \mathrm{H}-1^{\prime}\right), 5.32(\mathrm{~m}$, $\left.1 \mathrm{H}, \mathrm{CH}_{2} \mathrm{CH}=\mathrm{CH}\right), 5.40\left(\mathrm{dd}, J=3.3,11.2 \mathrm{~Hz}, 1 \mathrm{H}, \mathrm{H}-3^{\prime}\right)$, $5.52(\mathrm{~s}, 1 \mathrm{H}, \mathrm{CHPh}), 5.58(\mathrm{~s}, 1 \mathrm{H}, \mathrm{C} H \mathrm{Ph}), 5.96$ (dddd, $J=$ $\left.5.1,6.0,11.1,17.1 \mathrm{~Hz}, 1 \mathrm{H}, \mathrm{CH}_{2} \mathrm{CH}=\mathrm{CH}_{2}\right), 7.30-7.40(\mathrm{~m}$, $6 \mathrm{H}, \mathrm{Ar}-\mathrm{H}), 7.49(\mathrm{~m}, 2 \mathrm{H}, \mathrm{Ar}-\mathrm{H}), 7.55(\mathrm{~m}, 2 \mathrm{H}, \mathrm{Ar}-\mathrm{H}) ;{ }^{13} \mathrm{C}$ NMR $\left(126 \mathrm{MHz}, \mathrm{CDCl}_{3}\right) \delta 21.0,56.7,62.8,66.7,69.1$, 69.2, 69.4, 70.0, 72.3, 73.5, 76.8, $95.8\left(\mathrm{C}^{\prime} 1^{\prime}\right), 100.7,101.0$, 101.9 (C-1), 118.0, 126.1, 126.2, 128.0, 128.2, 128.8, 129.1, 133.8, 137.6, 170.4; FAB-HRMS $m / z$ calcd for $\mathrm{C}_{31} \mathrm{H}_{36} \mathrm{~N}_{3} \mathrm{O}_{11}$ $(\mathrm{M}+\mathrm{H})^{+}$626.2350, found 626.2372 .

\subsection{Comparative study}

4.6.1. TMSOTf-catalyzed glycosidation of 2-azido-2deoxyglucopyranosyl trichloroacetimidate $5 \alpha$ with alcohol 8 in acetonitrile (Table 9, entry 5). The glycosidation was performed according to the typical procedure $\left(1.5 \mathrm{~mL} \mathrm{MeCN},-40{ }^{\circ} \mathrm{C}, 0.3 \mathrm{~h}\right)$ employing trichloroacetimidate $5 \alpha^{7 \mathrm{~b}}(62.0 \mathrm{mg}, 0.10 \mathrm{mmol})$, alcohol $\mathbf{8}$ (51.1 mg, $0.11 \mathrm{mmol})$, TMSOTf $\left(1.0 \mathrm{M}\right.$ in $\mathrm{CH}_{2} \mathrm{Cl}_{2}, 0.015$ $\mathrm{mL}, 0.015 \mathrm{mmol})$, and pulverized molecular sieves $4 \mathrm{~A}(60$ $\mathrm{mg})$. An anomeric mixture of disaccharide 10 (47.2 $\mathrm{mg}$, $51 \%, \alpha: \beta=12: 88$ ) was obtained as a white solid from the crude product $(118.5 \mathrm{mg})$ after column chromatography (silica gel $6 \mathrm{~g}, 5: 1$ hexane/AcOEt with $1 \% \mathrm{Et}_{3} \mathrm{~N}$ ), along with $\beta$-trichloroacetamide $41(21.7 \mathrm{mg}, 35 \%)$ as a white solid.

Data for $N$-(2-azido-3,4,6-tri- $O$-benzyl-2-deoxy- $\beta$-D-glucopyranosyl)trichloroacetamide (41): ${ }^{26}$ TLC $R_{f}=0.59$ (2:1 hexane/AcOEt); mp 129.5-131.0 ${ }^{\circ} \mathrm{C}$ (colorless needles from $\mathrm{Et}_{2} \mathrm{O}$-hexane); $[\alpha]_{\mathrm{D}}{ }^{23}-3.79^{\circ}$ (c 1.00, $\mathrm{CHCl}_{3}$ ); IR
(KBr) 3360, 3032, 2893, 2108, 1699, 1520, 1454, 1363, 1277, 1128, $1060 \mathrm{~cm}^{-1} ;{ }^{1} \mathrm{H}$ NMR (500 MHz, $\left.\mathrm{CDCl}_{3}\right) \delta 3.46$ (dd, $J=9.4,9.6 \mathrm{~Hz}, 1 \mathrm{H}, \mathrm{H}-2), 3.54$ (ddd, $J=1.8,2.9,10.2$ $\mathrm{Hz}, 1 \mathrm{H}, \mathrm{H}-5), 3.62$ (dd, $J=8.9,9.6 \mathrm{~Hz}, 1 \mathrm{H}, \mathrm{H}-3), 3.71$ (dd, $J=1.8,11.0 \mathrm{~Hz}, 1 \mathrm{H}, \mathrm{H}-6 \mathrm{a}), 3.75(\mathrm{dd}, J=2.9,11.0 \mathrm{~Hz}, 1 \mathrm{H}$, H-6b), 3.79 (dd, $J=8.9,10.2 \mathrm{~Hz}, 1 \mathrm{H}, \mathrm{H}-4), 4.49$ (d, $J=$ $12.1 \mathrm{~Hz}, 1 \mathrm{H}, \mathrm{OCHPh}), 4.56(\mathrm{~d}, J=10.9 \mathrm{~Hz}, 1 \mathrm{H}, \mathrm{OCHPh})$, $4.60(\mathrm{~d}, J=12.1 \mathrm{~Hz}, 1 \mathrm{H}, \mathrm{OCHPh}), 4.80(\mathrm{~d}, J=10.9 \mathrm{~Hz}, 1 \mathrm{H}$, OCHPh), $4.88\left(\mathrm{~s}, 2 \mathrm{H}, \mathrm{OCH}_{2} \mathrm{Ph}\right), 4.97(\mathrm{dd}, J=9.3,9.4 \mathrm{~Hz}$, 1H, H-1), 7.08 (d, J=9.3 Hz, 1H, NH), 7.15 (m, 2H, Ar-H), 7.25-7.35 (m, 13H, Ar-H); ${ }^{13} \mathrm{C}$ NMR (126 MHz, $\left.\mathrm{CDCl}_{3}\right) \delta$ 65.8, 67.8, 73.6, 75.0, 75.7, 77.0, 77.2, 80.4 (C-1), 83.8, 92.0, 127.7, 127.89, 127.90, 128.0, 128.05, 128.14, 128.45, 128.51, 137.4, 137.5, 137.7, 161.7; FAB-HRMS $\mathrm{m} / \mathrm{z}$ calcd for $\mathrm{C}_{29} \mathrm{H}_{30} \mathrm{~N}_{4} \mathrm{O}_{5} \mathrm{Cl}_{3}(\mathrm{M}+\mathrm{H})^{+}$619.1282, found 619.1271; Anal. calcd for: $\mathrm{C}_{29} \mathrm{H}_{29} \mathrm{~N}_{4} \mathrm{O}_{5} \mathrm{Cl}_{3}$ : C, 56.19; $\mathrm{H}, 4.72 ; \mathrm{N}, 9.04$, found $\mathrm{C}, 56.04 ; \mathrm{H}, 4.62 ; \mathrm{N}, 8.89$.

4.6.2. TMSOTf-promoted glycosidation of 2-azido-2deoxyglucopyranosyl trichloroacetimidate $5 \alpha$ with alcohol 8 in propionitrile (Table 10, entry 3). The glycosidation was performed according to the typical procedure $\left(1.5 \mathrm{~mL}\right.$ EtCN, $\left.-78{ }^{\circ} \mathrm{C}, 0.3 \mathrm{~h}\right)$ employing trichloroacetimidate $5 \alpha^{7 \mathrm{~b}}(62.0 \mathrm{mg}, 0.10 \mathrm{mmol})$, alcohol $\mathbf{8}$ (51.1 mg, $0.11 \mathrm{mmol})$, TMSOTf (1.0 M in $\mathrm{CH}_{2} \mathrm{Cl}_{2}, 0.15$ $\mathrm{mL}, 0.15 \mathrm{mmol})$, and pulverized molecular sieves $4 \mathrm{~A}(60$ $\mathrm{mg})$. An anomeric mixture of disaccharide $10(49.6 \mathrm{mg}$, $54 \%, \alpha: \beta=7: 93$ ) was obtained as a colorless oil from the crude product $(125.4 \mathrm{mg})$ after column chromatography (silica gel $6 \mathrm{~g}, 5: 1$ hexane/AcOEt with $1 \% \mathrm{Et}_{3} \mathrm{~N}$ ), along with $\alpha$-amidine $\mathbf{4 2}$ (13.6 $\mathrm{mg}, 20 \%)$ as a colorless oil.

Data for $N$-(2-azido-3,4,6-tri- $O$-benzyl-2-deoxy- $\alpha$-D-glucopyranosyl)- $N$-(2,2,2-trichloro-1-iminoethyl)propionamide (42): TLC $R_{f}=0.50\left(2: 1\right.$ hexane/AcOEt); $[\alpha]_{\mathrm{D}}^{22}-11.4^{\circ}(c$ 1.61, $\left.\mathrm{CHCl}_{3}\right)$; IR $\left(\mathrm{CHCl}_{3}\right) 3020,2926,2868,2118,1635$, $1577,1221,1070 \mathrm{~cm}^{-1} ;{ }^{1} \mathrm{H}$ NMR $\left(500 \mathrm{MHz}, \mathrm{CDCl}_{3}\right) \delta 1.25$ (dd, $\left.J=7.4,7.5 \mathrm{~Hz}, 3 \mathrm{H}, \mathrm{CH}_{2} \mathrm{CH}_{3}\right), 2.61\left(\mathrm{~m}, 2 \mathrm{H}, \mathrm{CH}_{2} \mathrm{CH}_{3}\right.$ ), 3.61 (brd, $J=10.8 \mathrm{~Hz}, 1 \mathrm{H}, \mathrm{H}-6 \mathrm{a}), 3.66$ (m, 1H, H-5), 3.76 $(\mathrm{dd}, J=3.4,10.8 \mathrm{~Hz}, 1 \mathrm{H}, \mathrm{H}-6 \mathrm{~b}), 3.79$ (dd, $J=8.2,9.4 \mathrm{~Hz}$, $1 \mathrm{H}, \mathrm{H}-4), 3.82$ (dd, $J=8.2,9.5 \mathrm{~Hz}, 1 \mathrm{H}, \mathrm{H}-3), 3.89$ (dd, $J=$ $4.9,9.5 \mathrm{~Hz}, 1 \mathrm{H}, \mathrm{H}-2), 4.47$ (d, $J=12.0 \mathrm{~Hz}, 1 \mathrm{H}, \mathrm{OCHPh})$, $4.54(\mathrm{~d}, J=10.9 \mathrm{~Hz}, 1 \mathrm{H}, \mathrm{OCHPh}), 4.59$ (d, $J=12.0 \mathrm{~Hz}, 1 \mathrm{H}$, OCHPh), $4.80(\mathrm{~d}, J=10.9 \mathrm{~Hz}, 1 \mathrm{H}, \mathrm{OCHPh}), 4.92(\mathrm{~d}, J=$ $10.3 \mathrm{~Hz}, 1 \mathrm{H}, \mathrm{OCHPh}), 4.94(\mathrm{~d}, J=10.3 \mathrm{~Hz}, 1 \mathrm{H}, \mathrm{OCHPh})$, $5.46(\mathrm{~d}, J=4.9 \mathrm{~Hz}, 1 \mathrm{H}, \mathrm{H}-1), 7.16(\mathrm{~m}, 2 \mathrm{H}, \mathrm{Ar}-\mathrm{H})$, $7.26-7.36\left(\mathrm{~m}, 13 \mathrm{H}\right.$, Ar-H), $11.0(\mathrm{br}, 1 \mathrm{H}, \mathrm{NH}) ;{ }^{13} \mathrm{C} \mathrm{NMR}$ $\left(126 \mathrm{MHz} \mathrm{CDCl}_{3}\right) \delta 10.6,26.9,62.0,68.0,72.0,73.6,75.1$, 76.1, 77.6, 78.8, 81.3, 95.8, 127.7, 127.86, 127.91, 128.0, $128.1,128.2,128.45,128.50,128.6,137.2,137.4,137.5$, 174.5, 179.8; FAB-HRMS $\mathrm{m} / \mathrm{z}$ calcd for $\mathrm{C}_{32} \mathrm{H}_{35} \mathrm{~N}_{5} \mathrm{O}_{5} \mathrm{Cl}_{3}$ $(\mathrm{M}+\mathrm{H})^{+}$674.1704, found 674.1691; Anal. calcd for: $\mathrm{C}_{32} \mathrm{H}_{34} \mathrm{~N}_{5} \mathrm{O}_{5} \mathrm{Cl}_{3}$ : C, 56.94; $\mathrm{H}, 5.08 ; \mathrm{N}, 10.39 ; \mathrm{Cl}, 15.76$, found: C, 57.34; H, 5.18; N, 10.31; Cl, 15.41.

4.6.3. TMSOTf-promoted glycosidation of 2-azido-2deoxygalactopyranosyl trichloroacetimidate $43 \alpha$ with alcohol 8 in propionitrile (Table 11, entry 1). The glycosidation was performed according to the typical procedure $\left(1.5 \mathrm{~mL} \mathrm{EtCN},-78{ }^{\circ} \mathrm{C}, 0.1 \mathrm{~h}\right)$ employing trichloroacetimidate $43 \alpha^{7 \mathrm{a}}$ (62.0 $\left.\mathrm{mg}, 0.10 \mathrm{mmol}\right)$, alcohol 8 
(51.1 mg, $0.11 \mathrm{mmol}$ ), TMSOTf ( $1.0 \mathrm{M}$ in $\mathrm{CH}_{2} \mathrm{Cl}_{2}, 0.15$ $\mathrm{mL}, 0.15 \mathrm{mmol})$, and pulverized molecular sieves $4 \mathrm{~A}$ (60 $\mathrm{mg})$. An anomeric mixture of disaccharide $27(43.8 \mathrm{mg}$, $48 \%, \alpha: \beta=4: 96)$ was obtained as a white solid from the crude product $(123.4 \mathrm{mg})$ after column chromatography (silica gel $6 \mathrm{~g}, 5: 1$ hexane/AcOEt with $1 \% \mathrm{Et}_{3} \mathrm{~N}$ ), along with $\beta$-trichloroacetamide $44(23.0 \mathrm{mg}, 37 \%)$ and $\alpha$ amidine 45 (4.5 $\mathrm{mg}, 7 \%)$ as colorless oils.

Data for $N$-(2-azido-3,4,6-tri- $O$-benzyl-2-deoxy- $\beta$-D-galactopyranosyl)trichloroacetamide (44): TLC $R_{f}=0.59$ (2:1 hexane/AcOEt); $[\alpha]_{\mathrm{D}}{ }^{24}+21.1^{\circ}$ (c 1.20, $\mathrm{CHCl}_{3}$ ); IR (film) 3323, 3032, 2872, 2114, 1724, 1520, 1454, 1361, 1286, $1101 \mathrm{~cm}^{-1} ;{ }^{1} \mathrm{H}$ NMR $\left(500 \mathrm{MHz}, \mathrm{CDCl}_{3}\right) \delta 3.56(\mathrm{dd}, J=2.8$, $9.8 \mathrm{~Hz}, 1 \mathrm{H}, \mathrm{H}-3), 3.56-3.61$ (m, 2H, H-6a, H-6b), 3.68 (t, $J$ $=6.6 \mathrm{~Hz}, 1 \mathrm{H}, \mathrm{H}-5), 3.86(\mathrm{dd}, J=9.5,9.8 \mathrm{~Hz}, 1 \mathrm{H}, \mathrm{H}-2)$, 4.00 (brd, $J=2.8 \mathrm{~Hz}, 1 \mathrm{H}, \mathrm{H}-4), 4.43(\mathrm{~d}, J=11.8 \mathrm{~Hz}, 1 \mathrm{H}$, OCHPh), $4.47(\mathrm{~d}, J=11.8 \mathrm{~Hz}, 1 \mathrm{H}, \mathrm{OCHPh}), 4.57(\mathrm{~d}, J=$ $11.1 \mathrm{~Hz}, 1 \mathrm{H}, \mathrm{OC} H \mathrm{Ph}), 4.69(\mathrm{~d}, J=11.6 \mathrm{~Hz}, 1 \mathrm{H}, \mathrm{OC} H \mathrm{Ph})$, $4.74(\mathrm{~d}, J=11.6 \mathrm{~Hz}, 1 \mathrm{H}, \mathrm{OCHPh}), 4.87(\mathrm{~d}, J=11.1 \mathrm{~Hz}, 1 \mathrm{H}$, OCHPh), $4.91(\mathrm{dd}, J=9.3,9.5 \mathrm{~Hz}, 1 \mathrm{H}, \mathrm{H}-1), 7.07(\mathrm{~d}, J=$ $9.3 \mathrm{~Hz}, 1 \mathrm{H}, \mathrm{NH}), 7.27-7.40(\mathrm{~m}, 15 \mathrm{H}, \mathrm{Ar}-\mathrm{H}) ;{ }^{13} \mathrm{C}$ NMR (126 MHz, $\mathrm{CDCl}_{3}$ ) \& 62.5, 67.7, 71.9, 72.4, 73.5, 75.0, 75.5, 80.4 (C-1), 81.6, 92.0, 127.87, 127.92, 128.1, 128.3, 128.35, 128.44, 128.5, 137.1, 137.5, 137.9, 161.6; FAB-HRMS m/z calcd for $\mathrm{C}_{29} \mathrm{H}_{30} \mathrm{~N}_{4} \mathrm{O}_{5} \mathrm{Cl}_{3} \quad(\mathrm{M}+\mathrm{H})^{+}$619.1282, found 619.1276; Anal. calcd for: $\mathrm{C}_{29} \mathrm{H}_{29} \mathrm{~N}_{4} \mathrm{O}_{5} \mathrm{Cl}_{3}$ : C, 56.19; $\mathrm{H}$, 4.72; N, 9.04, found C, 55.98; H, 4.69; N, 8.93.

Data for $N$-(2-azido-3,4,6-tri- $O$-benzyl-2-deoxy- $\alpha$-D-galactopyranosyl)- $N-(2,2,2$-trichloro-1-iminoethyl)propionamide (45): TLC $R_{f}=0.43(2: 1$ hexane/AcOEt $) ;[\alpha]_{\mathrm{D}}^{20}+4.32^{\circ}(c$ $1.05, \mathrm{CHCl}_{3}$ ); IR (film) 3342, 3032, 2874, 2118, 1637, $1574,1454,1367,1211,1080 \mathrm{~cm}^{-1}$; ${ }^{1} \mathrm{H}$ NMR $(500 \mathrm{MHz}$, $\left.\mathrm{CDCl}_{3}\right) \delta 1.22\left(\mathrm{t}, J=7.5 \mathrm{~Hz}, 3 \mathrm{H}, \mathrm{CH}_{2} \mathrm{CH}_{3}\right), 2.55(\mathrm{dq}, J=$ $\left.15.3,7.5 \mathrm{~Hz}, 1 \mathrm{H}, \mathrm{CHCH}_{3}\right), 2.59(\mathrm{dq}, J=15.3,7.5 \mathrm{~Hz}, 1 \mathrm{H}$, $\mathrm{CHCH}_{3}$ ), 3.50 (dd, $\left.J=6.1,9.2 \mathrm{~Hz}, 1 \mathrm{H}, \mathrm{H}-6 \mathrm{a}\right), 3.56$ (dd, $J=$ 7.1, 9.2 Hz, 1H, H-6b), 3.76-3.79 (m, 2H, H-3, H-5), 4.02 (brs, 1H, H-4), 4.34 (dd, $J=5.1,10.5 \mathrm{~Hz}, 1 \mathrm{H}, \mathrm{H}-2), 4.40$ (d, $J=11.7 \mathrm{~Hz}, 1 \mathrm{H}, \mathrm{OC} H \mathrm{Ph}), 4.45(\mathrm{~d}, J=11.7 \mathrm{~Hz}, 1 \mathrm{H}$, OCHPh), $4.51(\mathrm{~d}, J=11.2 \mathrm{~Hz}, 1 \mathrm{H}, \mathrm{OC} H \mathrm{Ph}), 4.77(\mathrm{~d}, J=$ $10.6 \mathrm{~Hz}, 1 \mathrm{H}, \mathrm{OCHPh}), 4.79(\mathrm{~d}, J=10.6 \mathrm{~Hz}, 1 \mathrm{H}, \mathrm{OCHPh})$, $4.57(\mathrm{~d}, J=11.2 \mathrm{~Hz}, 1 \mathrm{H}, \mathrm{OCHPh}), 5.44(\mathrm{~d}, J=5.1 \mathrm{~Hz}, 1 \mathrm{H}$, $\mathrm{H}-1), 7.24-7.42(\mathrm{~m}, 15 \mathrm{H}, \mathrm{Ar}-\mathrm{H}), 11.0(\mathrm{br}, 1 \mathrm{H}, \mathrm{NH}) ;{ }^{13} \mathrm{C}$ NMR $\left(126 \mathrm{MHz}, \mathrm{CDCl}_{3}\right) \delta 10.5,26.9,58.2,68.2,70.8$, 72.5, 72.7, 73.6, 75.0, 78.8, 79.0, 95.9, 127.85, 127.91, $128.0,128.1,128.2,128.4,128.5,128.6,136.8,137.4$, $137.9, \quad 174.4, \quad 180.1$; ESI-HRMS $\mathrm{m} / \mathrm{z}$ calcd for $\mathrm{C}_{32} \mathrm{H}_{34} \mathrm{~N}_{5} \mathrm{O}_{5} \mathrm{Cl}_{3} \mathrm{Na}(\mathrm{M}+\mathrm{Na})^{+}$696.1523, found 696.1537.

\section{Acknowledgments}

This research was supported in part by a Grant-in-Aid for Scientific Research on Priority Areas (A) "Exploitation of Multi-Element Cyclic Molecules" from the Ministry of Education, Culture, Sports, Science and Technology, Japan. T.T. is grateful to JSPS for a graduate fellowship. We thank Ms. H. Matsumoto, A. Maeda, S. Oka, and M. Kiuchi of the Center for Instrumental Analysis, Hokkaido University, for technical assistance in MS and elemental analysis.

\section{References}

1. For recent reviews see: (a) Toshima, K.; Tatsuta, K. Chem. Rev. 1993, 93, 1503-1531. (b) Boons, G.-J. Tetrahedron 1996, 52, 1095-1121. (c) Modern Methods in Carbohydrate Synthesis; Khan, S. H., O'Neil, R. A., Eds.; Harwood Academic Publishers: Amsterdam, 1996. (d) Carbohydrates in Chemistry and Biology; Ernst, B., Hart, G. W., Sinaÿ, P. Eds.; Wiley-VCH: Weinheim, 2000; Part I. (e) Davis, B. G. $J$. Chem. Soc., Perkin Trans. 1 2000, 2137-2160. (f) Demchenko, A. V. Synlett 2003, 1225-1240.

2. For reviews, see: (a) Banoub, J.; Boullanger, P.; Lafont, D. Chem. Rev. 1992, 92, 1167-1195. (b) Debenham, J.; Rodebaugh, R.; Fraser-Reid, B. Liebigs Ann./Recueil 1997, 791-802.

3. For a review, see: Stöckl, W. P.; Weidmann, H. J. Carbohydr. Chem. 1989, 8, 169-198.

4. Lemieux, R. U.; Takeda, T.; Chung, B. Y. ACS Symp. Ser. 1976, 39, 90-115.

5. (a) Imoto, M.; Yoshimura, H.; Shimamoto, T.; Sakaguchi, N.; Kusumoto, S.; Shiba, T. Bull. Chem. Soc. Jpn. 1987, 60, 2205-2214. (b) Ellervik, U.; Magnusson, G. Carbohydr. Res. 1996, 280, 251-260. (c) Boullanger, P.; Jouineau, M.; Bouammali, B.; Lafont, D.; Descotes, G. Carbohydr. Res. 1990, 202, 151-164. (d) Blatter, G.; Beau, J.; Jacquinet, J. Carbohydr. Res. 1994, 260, 189-202. (e) Debenham, J. S.; Madsen, R.; Roberts, C.; Fraser-Reid, B. J. Am. Chem. Soc. 1995, 117, 3302-3303. (f) Castro-Palomino, J. C.; Schmidt, R. R. Tetrahedron Lett. 1995, 36, 5343-5346. (g) Debenham, J. S.; Debenham, S. D.; Fraser-Reid, B. Bioorg. Med. Chem. 1996, 4, 1909-1918. (h) Meinjohanns, E.; Meldal, M.; Paulsen, H.; Bock, K. J. Chem. Soc., Perkin Trans. 1 1995, 405-415. (i) Jensen, K. J.; Hansen, P. R.; Venugopal, D.; Barany, G. J. Am. Chem. Soc. 1996, 118, 3148-3155. (j) Castro-Palomino, J. C.; Schmidt, R. R. Tetrahedron Lett. 1995, 36, 6871-6874. (k) Shimizu, H.; Ito, Y.; Matsuzaki, Y.; Iijima, H.; Ogawa, T. Biosci. Biotechnol. Biochem. 1996, 60, 73-76. (1) Aly, M. R. E.; Castro-Palomino, J. C.; Ibrahim, E.S. I.; El-Ashry, E.-S. H.; Schmidt, R. R. Eur. J. Org. Chem. 1998, 2305-2316. (m) Jiao, H.; Hindsgaul, O. Angew. Chem., Int. Ed. 1999, 38, 346-348. (n) Castro-Palomino, J. C.; Schmidt, R. R. Tetrahedron Lett. 2000, 41, 629-632.

6. For reviews, see: (a) Schmidt, R. R. Angew. Chem., Int. Ed. Engl. 1986, 25, 212-235. (b) Schmidt, R. R.; Kinzy, W. Adv. Carbohydr. Chem. Biochem. 1994, 50, 21-123. (c) Schmidt, R. R.; Jung, K.-H. In Preparative Carbohydrate Chemistry, Hanessian, S. Ed. Marcel Dekker: New York, 1997; pp. 283-312. (d) Schmidt, R. R.; Jung, K.-H. In Carbohydrates in Chemistry and Biology, Ernst, B.; Hart, G. W.; Sinaÿ, P. Eds. Wiley-VCH: Weinheim, 2000; pp. 5-59.

7. (a) Grundler, G.; Schmidt, R. R. Liebigs Ann. Chem. 1984, 1826-1847. (b) Kinzy, W.; Schmidt, R. R. Liebigs Ann. Chem. 1985, 1537-1545. High levels of $\beta$-selectivity achieved by this method are mostly limited to the use of $\alpha$ trichloroacetimidates. See also Refs $6 a$ and $6 b$.

8. Schmidt, R. R.; Behrendt, M.; Toepfer, A. Synlett 1990, 694-696. In this paper, it was demonstrated that $\beta$ selectivities are practically independent of the anomeric configuration of the starting trichloroacetimidates.

9. Marra, A.; Gauffeny, F.; Sinä̈, P. Tetrahedron 1991, 47, 5149-5160.

10. Marra, A.; Esnault, J.; Veyrières, A.; Sinä̈, P. J. Am. Chem. Soc. 1992, 114, 6354-6360.

11. Furukawa, H.; Koide, K.; Takao, K.; Kobayashi, S. Chem. Pharm. Bull. 1998, 46, 1244-1247.

12. (a) Plante, O. J.; Palmacci, E. R.; Seeberger, P. H. Org. Lett. 2000, 2, 3841-3843. (b) Plante, O. J.; Palmacci, E. R.; 
Andrade, R. B.; Seeberger, P. H. J. Am. Chem. Soc. 2001, 123, 9545-9554.

13. Codée, J. D.; Litjens, R. E. J. N.; den Heeten, R.; Overkleeft, H. S.; van Boom, J. H.; van der Marel, G. A. Org. Lett. 2003, $5,1519-1522$.

14. For methods involving azaglycosidation of glycals, see: (a) Griffith, D. A.; Danishefsky, S. J. J. Am. Chem. Soc. 1990, 112, 5811-5819. (b) Di Bussolo, V.; Liu, J.; Huffman, L. G., Jr.; Gin, D. Y. Angew. Chem., Int. Ed. 2000, 39, 204-207. (c) Liu, J.; Gin, D. Y. J. Am. Chem. Soc. 2002, 124, 9789-9797.

15. (a) Hashimoto, S.; Honda, T.; Ikegami, S. J. Chem. Soc., Chem. Commun. 1989, 685-687. (b) Hashimoto, S.; Honda, T.; Ikegami, S. Heterocycles 1990, 30, 775-778. (c) Hashimoto, S.; Honda, T.; Ikegami, S. Tetrahedron Lett. 1990, 31, 4769-4772. (d) Hashimoto, S.; Yanagiya, Y.; Honda, T.; Harada, H.; Ikegami, S. Tetrahedron Lett. 1992, 33, 3523-3526. (e) Hashimoto, S.; Umeo, K.; Sano, A.; Watanabe, N.; Nakajima, M.; Ikegami, S. Tetrahedron Lett. 1995, 36, 2251-2254. (f) Hashimoto, S.; Sano, A.; Sakamoto, H.; Nakajima, M.; Yanagiya, Y.; Ikegami, S. Synlett 1995, 1271-1273. (g) Tanaka, H.; Sakamoto, H.; Sano, A.; Nakamura, S.; Nakajima, M.; Hashimoto, S. Chem. Commun. 1999, 1259-1260. (h) Tsuda, T.; Sato, S.; Nakamura, S.; Hashimoto, S. Heterocycles 2003, 59, 509-515. See also: http://www.glycoforum.gr.jp/science/word/glycotechnology/ GT-A01E.html.

16. For a preliminary communication, see: Tsuda, T.; Nakamura, S.; Hashimoto, S. Tetrahedron Lett. 2003, 44, 6453-6457.

17. Sabesan, S.; Neira, S. Carbohydr. Res. 1992, 223, 169-185.

18. Schmidt, R. R.; Stumpp, M. Liebigs Ann. Chem. 1984, 23, 680-691.

19. (a) Hashimoto, S.; Sakamoto, H.; Honda, T.; Ikegami, S. Tetrahedron Lett. 1997, 38, 5181-5184. (b) Hashimoto, S.; Sakamoto, H.; Honda, T.; Abe, H.; Nakamura, S.; Ikegami, S. Tetrahedron Lett. 1997, 38, 8969-8972. (c) Sakamoto, H.; Nakamura, S.; Tsuda, T.; Hashimoto, S. Tetrahedron Lett. 2000, 41, 7691-7695.

20. (a) Fraser-Reid, B.; Wu, Z.; Andrews, C. W.; Skowronski, E.; Bowen, J. P. J. Am. Chem. Soc. 1991, 113, 1434-1435. (b) Fraser-Reid, B.; Udodong, U. E.; Wu, Z.; Ottosson, H.; Merritt, J. R.; Rao, C. S.; Roberts, C.; Madsen, R. Synlett 1992, 927-942. (c) Douglas, N. L.; Ley, S. V.; Lücking, U.; Warriner, S. L. J. Chem. Soc., Perkin Trans. 1 1998, 51-65. (d) Zhang, Z.; Ollmann, I. R.; Ye, X.-S.; Wischnat, R.; Baasov, T.; Wong, C.-H. J. Am. Chem. Soc. 1999, 121, 734-753.

21. (a) Crich, D.; Sun, S. J. Org. Chem. 1996, 61, 4506-4507. (b) Crich, D.; Sun, S. J. Org. Chem. 1997, 62, 1198-1199. (c) Crich, D.; Sun, S. J. Am. Chem. Soc. 1997, 119, 11217-11223. (d) Crich, D.; Sun, S. Tetrahedron 1998, 54, 8321-8348. (e) Crich, D.; Cai, W. J. Org. Chem. 1999, 64, 4926-4930.

22. Schmidt and Lassaletta and co-workers reported that TMSOTf-catalyzed glycosidation of the imidate corresponding to $\mathbf{4 c}$ with diol $\mathbf{3 4}$ in acetonitrile at $-20{ }^{\circ} \mathrm{C}$ proceeded regio- and stereoselectively, affording $\beta$-linked disaccharide $\mathbf{4 0} \beta$ as a single isomer in $84 \%$ yield. However, we found that disaccharides $40 \alpha$ and $40 \beta$ exhibits different $R_{f}$ values (0.44 and 0.36 , respectively, performed using $3: 1$ toluene/acetone). The reaction of the imidate with diol $\mathbf{3 4}$ in the presence of 0.05 equiv of TMSOTf gave $\mathbf{4 0}$ in $80 \%$ yield with an $\alpha: \beta$ ratio of 5:95. (a) Lassaletta, J. M.; Schmidt, R. R. Tetrahedron Lett. 1995, 36, 4209-4212. (b) Lassaletta, J. M.; Carlsson, K.; Garegg, P. J.; Schmidt, R. R. J. Org. Chem. 1996, 61, 6873-6880.

23. Very recently, Bols and co-workers reported that electronic effects also play a substantial role in the disarming effect of the 4,6-acetal group: Jensen, H. H.; Nordstrøm, L. U.; Bols, M. J. Am. Chem. Soc. 2004, 126, 9205-9213.

24. (a) Toepfer, A.; Schmidt, R. R. Tetrahedron Lett. 1992, 33, 5161-5164. (b) Greilich, U.; Zimmermann, P.; Jung, K.-H.; Schmidt, R. R. Liebigs Ann. Chem. 1993, 859-864. (c) Toepfer, A.; Kinzy, W.; Schmidt, R. R. Liebigs Ann. Chem. 1994, 449-464. (d) Greilich, U.; Brescello, R.; Jung, K.-H.; Schmidt, R. R. Liebigs Ann. Chem. 1996, 663-672. (e) Lassaletta, J. M.; Schmidt, R. R. Liebigs Ann. Chem. 1996, 1417-1423. (f) Hummel, G.; Schmidt, R. R. Tetrahedron Lett. 1997, 38, 1173-1176. (g) Bourhis, P.; Machetto, F.; Duchaussoy, P.; Hérault, J.-P.; Mallet, J.-M.; Herbert, J.-M.; Petitou, M.; Sinaÿ, P. Bioorg. Med. Chem. Lett. 1997, 7, 2843-2846. (h) Weiler, S.; Schmidt, R. R. Tetrahedron Lett. 1998, 39, 2299-2302. (i) Tietze, L. F.; Janssen, C. O.; Gewert, J.-A. Eur. J. Org. Chem. 1998, 1887-1894.

25. (a) Ley, S. V.; Yeung, L. L. Synlett 1992, 997-998. (b) Cottaz, S.; Brimacombe, J. S.; Ferguson, M. A. J. Carbohydr. Res. 1995, 270, 85-91.

26. For examples of the formation of $N$-glycosyl trichloroacetamides as byproducts, see: (a) Sadozai, K. K.; Nukada, T.; Ito, Y.; Nakahara, Y.; Ogawa, T.; Kobata, A. Carbohydr. Res. 1986, 157, 101-123. (b) Kinzy, W.; Schmidt, R. R. Liebigs Ann. Chem. 1987, 407-415. (c) Nunomura, S.; Ogawa, T. Tetrahedron Lett. 1988, 29, 5681-5684. (d) Kobayashi, M.; Yamazaki, F.; Ito, Y.; Ogawa, T. Carbohydr. Res. 1990, 201, 51-67. (e) Lindberg, J.; Öhberg, L.; Garegg, P.; Konderson, P. Tetrahedron 2002, 58, 1387-1398. (f) Lohman, G. J. S.; Seeberger, P. H. J. Org. Chem. 2004, 69, 4081-4093.

27. (a) Macindoe, W. M.; Ijima, H.; Nakahara, Y.; Ogawa, T. Carbohydr. Res. 1995, 269, 227-257. (b) Plé, K. Carbohydr. Res. 2003, 338, 1441-1454.

28. Urban, D.; Skrydstrup, T.; Beau, J.-M. J. Org. Chem. 1998, 63, 2507-2516.

29. Similar to diphenyl phosphates, all attempts to employ trichloroacetimidates that correspond to $\mathbf{2 b}, \mathbf{2 c}$ and $\mathbf{4 b}$ failed.

30. Hashimoto, S.; Hayashi, M.; Noyori, R. Tetrahedron Lett. 1984, 25, 1379-1382.

31. Ratcliffe, A. J.; Fraser-Reid, B. J. Chem. Soc., Perkin Trans. 1 1990, 747-750.

32. Before Ratcliffe and Fraser-Reid characterized the product obtained from the reaction of $\alpha$-nitrilium ion with 2chlorobenzoic acid, the intermediacy of the nitrilium ion or nitrilium-nitrile conjugates were advocated. (a) Pougny, J.R.; Sinaÿ, P. Tetrahedron Lett. 1976, 4073-4076. (b) Lemieux, R. U.; Ratcliffe, R. M. Can. J. Chem. 1979, 57, 1244-1251. (c) Schmidt, R. R.; Rücker, E. Tetrahedron Lett. 1980, 21, 1421-1424. (d) Pavia, A. A.; Ung-Chhun, S. N.; Durand, J.-L. J. Org. Chem. 1981, 46, 3158-3160. (e) Schmidt, R. R.; Michel, J. J. Carbohydr. Chem. 1985, 4, 141-169.

33. Good to high $\beta$-selectivities observed in $\mathrm{CH}_{2} \mathrm{Cl}_{2}$ or toluene would be attributed to the intermediacy of the thermodynamically more stable $\alpha$-glycosyl triflate followed by the backside attack of acceptor alcohols on this intermediate. See Ref 21c.

34. (a) Deslongchamps, P. Organic Chemistry Series, Vol. 1: Stereoelectronic Effects in Organic Chemistry; Pergamon: Oxford, UK, 1983. (b) Kirby, A. J. The Anomeric Effect and Related Stereoelectronic Effects at Oxygen; Springer Verlag, Berlin, 1983.

35. It was believed as the reverse anomeric effect (RAE) that cationic substituents on a pyranose ring have the tendency to take an equatorial position. However, Perrin and co-workers recently reexamed this and their observations are the exact opposite to what is expected from RAE (i.e. consistent with 
an enhancement of the normal anomeric effect) and suggest that previous evidence for this effect is unreliable. (a) Perrin, C. L. Tetrahedron 1995, 51, 11901-11935. (b) Perrin, C. L.; Fabian, M. A.; Brunckova, J.; Ohta, B. K. J. Am. Chem. Soc. 1999, 121, 6911-6918. (c) Perrin, C. L.; Kuperman, J. J. Am. Chem. Soc. 2003, 125, 8846-8851.
36. Paulsen, H.; Sumfleth, B. Chem. Ber. 1979, 112, 3203-3213.

37. Gauffeny, F.; Marra, A.; Shi Shun, L. K.; Sinaÿ, P.; Tabeur, C. Carbohydr. Res. 1991, 219, 237-240.

38. Orgueira, H. A.; Bartolozzi, A.; Schell, P.; Litjens, R. E. J. N.; Palmacci, E. R.; Seeberger, P. H. Chem. Eur. J. 2003, 9, 140-169. 